\title{
Natural Convection in Horizontal Fluid-Superposed Porous Layers Heated Locally From Below
}

\author{
A DISSERTATION \\ SUBMITTED TO THE FACULTY OF THE GRADUATE SCHOOL \\ OF THE UNIVERSITY OF MINNESOTA \\ BY
}

Aniruddha Bagchi

IN PARTIAL FULFILLMENT OF THE REQUIREMENTS

FOR THE DEGREE OF

DOCTOR OF PHILOSOPHY

Francis A. Kulacki, Adviser

December, 2010 
(C) Aniruddha Bagchi 2010

ALL RIGHTS RESERVED 


\section{Acknowledgements}

I would like to take this opportunity to express my sincere appreciation to Prof. Francis A. Kulacki for his constant encouragement and motivation, and for his invaluable help and advice during the course of this study. His guidance was a key factor in keeping me on track during a difficult stage of my academic career, and I am very thankful to him for that.

I would also like to thank Prof. Terry Simon and Dr. Wojciech Lipiński of the Mechanical Engineering Department and Prof. Vaughan Voller of the Civil Engineering Department for taking the time to serve on my committee and for critically evaluating this manuscript.

Finally there are several people who deserve my appreciation for making this rocky journey of mine through graduate school a little more tolerable. My wife, Basabdatta, deserves special mention for being my best friend, my most ardent supporter and a constant source of inspiration during my doctoral degree. My parents deserve my utmost gratitude for supporting me throughout my academic career and giving me the freedom of choice. I would also like to thank my sister and her family for the support that they have provided me with during my stay in the United States. Thanks must also be given to my friends here in the U.S. and back home in India who have been by my side and have been a constant

source of encouragement to me. Last, but not the least, I am forever indebted to my late grandfather, Mr. Manoranjan Bagchi, who was and will be the best teacher I ever had. 


\section{Dedication}

To my parents, Shyamali and Tapas Kumar Bagchi. 


\begin{abstract}
Natural convection in a horizontal fluid-superposed porous layer heated locally from below is studied in this thesis. This problem occurs in numerous engineering and geophysical systems, such as fibrous and granular thermal insulations, water reservoirs, grain storage installations, solid-matrix heat exchangers, solidifying castings, and post-accident cooling of nuclear reactors. In nature, thermal circulation in lakes, shallow coastal areas and other reservoirs occurs in a system having a fluid layer superposed on top of a porous matrix that is heated locally at the base. In spite of its fundamental nature, there are virtually no studies in the literature pertaining to this problem. To address this shortcoming, the fundamental aspects of this problem are studied in this work.

The goal of the thesis is to study how free convective heat transfer in a composite layer with a localized bottom heat source is affected by parameters such as the size of the heat source, the fluid-to-porous layer height ratio, the Darcy number, the aspect ratio, the solid-to-fluid conductivity ratio, and the fluid Prandtl number. To that end, two particular aspects of the problem are studied: (a) the steady-state and transient flow and temperature fields, and (b) measurement and prediction of the overall heat transfer characteristics of the system.

A numerical approach has been used to study the development of the temperature and flow fields in the system and predict overall heat transfer rates. A one-domain formulation, which uses a single set of governing equations to model fluid flow and heat transfer throughout the entire composite domain, is used. To solve the governing equations, a control volume based numerical solution technique is used. Results show that the nature of convective motion in a composite system with a localized bottom heat source is identical to that observed when the base is uniformly heated. A cellular flow pattern is observed with flow penetration occurring from the overlying fluid layer to the underlying porous layer. Penetration, however, is significant only near the fluid-porous layer interface. The overall heat transfer coefficients are found to depend strongly on the heater length, height ratio, and the fluid-to-porous layer conductivity ratio, while the effects of the aspect ratio and the fluid Prandtl number are not very significant. The effect of the Darcy number is moderate up to Darcy numbers of $10^{-3}$ beyond which there is a sudden increase in heat transfer coefficients.
\end{abstract}

Experiments are performed to validate the numerical solutions and develop empirical Nusselt-versus-Rayleigh number correlations. Experiments are conducted in a cubical 
chamber with $3 \mathrm{~mm}$ DIA glass beads as the porous layer and distilled water as the saturating fluid. Two different heater lengths and three different height ratios are investigated. Experimental results confirm the numerical predictions that the Nusselt number increases with a decrease in the heater length and an increase in the height ratio. However, significant numerical differences are seen when experimental and numerical results are compared. The most likely reason for the observed discrepancy is the implementation of the one-domain model for the numerical solution. This discrepancy calls into question the results of prior numerical studies for the fully heated bottom which have used a one-domain formulation and emphasizes the necessity of validation via experiment. 


\section{Contents}

Acknowledgements $\quad$ i

Dedication $\quad$ ii

Abstract $\quad$ iii

List of Tables viii

List of Figures $\quad x$

Nomenclature $\quad$ xvi

1 Introduction 1

1.1 Background . . . . . . . . . . . . . . . . . . . . 1

1.2 Aims and objectives of the thesis . . . . . . . . . . . 4

1.3 Structure and organization of the thesis . . . . . . . . . . 5

2 Literature Review $\quad 6$

2.1 Natural convection in horizontal porous layers heated uniformly . . . . . . . 6

2.2 Natural convection in horizontal porous layers heated locally . . . . . . . . 15

2.3 Convection in fluid-superposed porous layers heated uniformly . . . . . . 23

2.4 Closure . . . . . . . . . . . . . . . . . . . . . . 33

3 Mathematical Formulation and Numerical Method 34

3.1 Mathematical Formulation . . . . . . . . . . . . . . . . . . . . . 34

3.1.1 Governing equations . . . . . . . . . . . . . . . . 34

3.1 .2 Boundary Conditions . . . . . . . . . . . . . . . . . 38

3.1.3 One-domain Formulation . . . . . . . . . . . . . . 40

3.2 Numerical solution of the governing equations . . . . . . . . . . . . . . 43

3.2.1 Derivation of the finite difference equations . . . . . . . . . . . . 43

3.2.1.1 Stream function-vorticity equation . . . . . . . . . . . 43 
3.2.1.2 Vorticity transport and energy equations . . . . . . . . 45

3.2.1.3 The QUICK scheme . . . . . . . . . . . . . . 50

3.2.1.4 Calculation of the Velocities . . . . . . . . . . 52

3.2.1.5 Treatment of Boundary Conditions . . . . . . . . . . 53

3.2.2 Solution of the discretization equations . . . . . . . . . . . 56

3.2 .3 General solution algorithm . . . . . . . . . . . . . 57

3.3 Post-processing of data . . . . . . . . . . . . . . . . . 58

3.4 Convergence and accuracy of the numerical scheme . . . . . . . . . . . 59

3.4.1 Convergence criterion . . . . . . . . . . . . . . . 59

3.4.2 Relaxation parameter . . . . . . . . . . . . . . . . 60

3.4.3 Grid convergence . . . . . . . . . . . . . . . . . . 61

3.4 .4 Energy balance . . . . . . . . . . . . . . . . . . . 61

4 Numerical Results $\quad 63$

4.1 Convergence, accuracy and stability of the numerical scheme . . . . . . 63

4.2 Verification of the numerical scheme . . . . . . . . . . . . . 67

4.3 Convection in fluid-superposed porous layers heated locally . . . . . . . . . 71

4.3.1 Effect of the heater-to-base length ratio . . . . . . . . . . . . 71

4.3.2 Effect of the porous layer-to-total height ratio . . . . . . . . . . 80

4.3 .3 Effect of the Darcy number . . . . . . . . . . . . . . . 86

4.3 .4 Effect of the aspect ratio . . . . . . . . . . . . . . 92

4.3.5 Effect of the solid-to-fluid conductivity ratio . . . . . . . . . . . . . 99

4.3.6 Effect of the Prandtl number . . . . . . . . . . . . . . . . 105

4.4 Evolution of the flow and temperature fields . . . . . . . . . . . . 108

4.5 Conclusions . . . . . . . . . . . . . . . . . . . . . . 110

5 Experimental Design and Procedure $\quad 114$

5.1 Design of experiments . . . . . . . . . . . . . . . . . . . . . 114

5.2 Design of experimental setup . . . . . . . . . . . . . . 117

5.2.1 Experimental apparatus . . . . . . . . . . . . . . . . . . . . 118

5.2.1.1 Convection cell . . . . . . . . . . . . . . . . . . 119

5.2.1.2 Base plate assembly . . . . . . . . . . . . . . 119

5.2 .1 .3 Top plate assembly . . . . . . . . . . . . . . . . . 122

5.2 .2 Instrumentation and assembly $\ldots \ldots \ldots \ldots$. . . . . . . . . 124

$5.3 \quad$ Experimental procedure . . . . . . . . . . . . . . . . . . . 129 
6 Experimental Results $\quad 132$

6.1 Data analysis procedure . . . . . . . . . . . . . . . 132

6.1.1 Data reduction procedure . . . . . . . . . . . . . 132

6.1.2 Estimation of heat losses . . . . . . . . . . . . . . . 133

6.2 Experimental Results . . . . . . . . . . . . . . . . . . . 137

6.2.1 Validation of the experimental setup . . . . . . . . . . 137

6.2 .2 Heat transfer results . . . . . . . . . . . . . . . . . . . . 140

6.2 .3 Temperature profiles . . . . . . . . . . . . . 146

6.3 Conclusions . . . . . . . . . . . . . . . . . . . . . 148

7 Conclusions $\quad 149$

7.1 Comparison of numerical and experimental results . . . . . . . . . . 149

7.1.1 Discrepancies due to differences in boundary conditions . . . . . . . 151

7.1.2 Effect of the mathematical model . . . . . . . . . . . . . . . 154

7.2 Contributions of the present study . . . . . . . . . . . . . . . . 159

7.3 Recommendations for future work . . . . . . . . . . . . . . 161

$\begin{array}{ll}\text { References } & 163\end{array}$

$\begin{array}{lll}\text { Appendix A. Direct solver for the linear equations } & 171\end{array}$

$\begin{array}{ll}\text { Appendix B. Data Tables } & 173\end{array}$

$\begin{array}{ll}\text { Appendix C. Thermophysical Properties } & 179\end{array}$

$\begin{array}{ll}\text { Appendix D. Uncertainty Analysis } & 181\end{array}$

D.1 Uncertainty in Nusselt number . . . . . . . . . . . . . . . . . . 181

D.2 Uncertainty in Rayleigh number . . . . . . . . . . . . . . . . . . 182

D.3 Uncertainty in thermophysical constants . . . . . . . . . . . . . . . . 182

D.4 Uncertainty in length and area measurements . . . . . . . . . . . . . 183

D.5 Uncertainty in thermocouple measurements . . . . . . . . . . . . . 183

D.6 Uncertainty in $\Delta T \ldots \ldots \ldots \ldots \ldots \ldots \ldots \ldots \ldots \ldots$

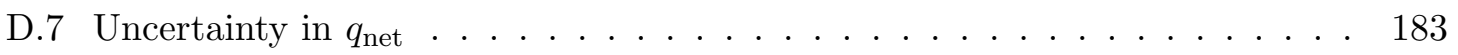




\section{List of Tables}

2.1 The various thermal boundary conditions used for the local heating problem. . . . . . . . . . . . . . . 17

4.1 Parameters used in the simulations for code validation. . . . . . . . . 68

4.2 Values of the parameters used in the various simulations. . . . . . . . . 73

5.1 Overview of the experimental design selected. . . . . . . . . . . 117

5.2 Thermocouple locations at the different surfaces. . . . . . . . . . . . 125

6.1 Parameters used for studies on validation of experimental setup. . . . . . . . . . . . . . . . . . . 138

6.2 Measured parameters for all experiments. . . . . . . . . . . . . . . . . 141

6.3 Reduced heat transfer data for all experiments. . . . . . . . . . . . . . . . 142

6.4 Curve fit parameters for all the experiments. . . . . . . . . . . . . . 146

7.1 Comparison of experimental and numerical results. . . . . . . . . . . 151

7.2 Temperature distribution on the heater surface for $\eta=0.75$,

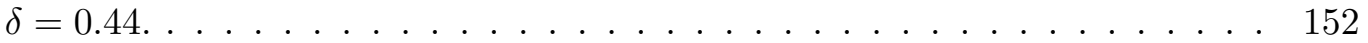

7.3 Comparison of numerical simulations with data of Prasad and $\operatorname{Tian}[24] \ldots \ldots \ldots \ldots \ldots \ldots \ldots$

7.4 Parameters used in the different numerical studies compared. . . . . . . . . . . . . . . . . . 157

7.5 Parameters used in the different experimental studies compared. . . . . . . . . . . . . . . . . . 160

A.1 Packages used in the different steps of the direct solution. . . . . . . . . . 172

B.1 Numerical Nusselt-Rayleigh number data for different values

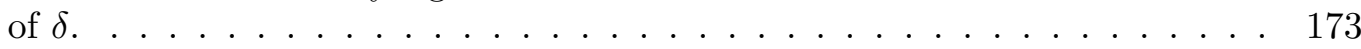

B.2 Numerical Nusselt-Rayleigh number data for different values of $\eta$. . . . . . . . . . . . . . . . . . . . . . . . . . . . 174 
B.3 Numerical Nusselt-Rayleigh number data for different values of $A$. . . . . . . . . . . . . . . . . . . . . . . 174

B.4 Numerical Nusselt-Rayleigh number data for different values

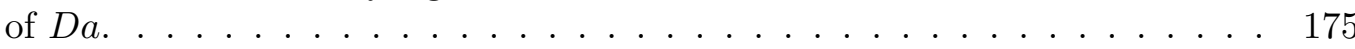

B.5 Numerical Nusselt-Rayleigh number data for different values of $\lambda \ldots \ldots \ldots \ldots \ldots \ldots \ldots \ldots \ldots$

B.6 Numerical Nusselt-Rayleigh number data for different values of $\mathrm{Pr} . \ldots \ldots \ldots \ldots \ldots \ldots$

B.7 Measured experimental parameters for $\delta=0.44$ and $\eta=0.5 \ldots \ldots$. . . 177

B.8 Measured experimental parameters for $\delta=0.44$ and $\eta=0.67 \ldots \ldots \ldots$.

B.9 Measured experimental parameters for $\delta=0.44$ and $\eta=0.75 \ldots \ldots \ldots . .177$

B.10 Measured experimental parameters for $\delta=0.11$ and $\eta=0.5 \ldots \ldots . . .178$

C.1 Thermophysical properties of solid materials. . . . . . . . . . . . 179

C.2 Properties of water for experiment with $\delta=0.44$ and $\eta=0.5 \ldots \ldots \ldots$

C.3 Properties of water for experiment with $\delta=0.44$ and $\eta=0.67 . \ldots . . . \quad 180$

C.4 Properties of water for experiment with $\delta=0.44$ and $\eta=0.75 \ldots \ldots$. . . 180

C.5 Properties of water for experiment with $\delta=0.11$ and $\eta=0.5 \ldots \ldots \ldots \ldots$ 


\section{List of Figures}

1.1 Schematic diagram of (a) an idealized saturated porous medium, and (b) a naturally occurring porous media. . . . . . . . . . . . . . .

2.1 Schematic of the basic problem of natural convection in a

horizontal porous layer heated uniformly from below. . . . . . . . . . . .

2.2 Compilation of experimental results of Nusselt number versus Rayleigh number for convection in a horizontal porous layer heated uniformly from below (Aichlmayr $[27]$ ). . . . . . . . . . .

2.3 Two-dimensional domain of convection in a horizontal porous layer heated locally from below. . . . . . . . . . . . . . . . . . .

2.4 Isotherms (left column) and streamlines (right column) in a horizontal porous layer of aspect ratio $A=10$ and Rayleigh number $R a_{\mathrm{m}}=80$ for various values of the dimensionless heater length $\delta_{1}$. (a) and (b), $\delta_{1}=2 ;(\mathrm{c})$ and (d), $\delta_{1}=3 ;(\mathrm{e})$ and (f), $\delta_{1}=4 ;(\mathrm{g})$ and $(\mathrm{h}), \delta_{1}=6 .($ Elder $[29]) \ldots \ldots \ldots \ldots$

2.5 Computed isotherms during a single oscillation for a localized heating problem in a porous layer of aspect ratio $A=2$, $R a_{\mathrm{m}}=750$, heater length $\delta_{1}=0.5$ (Horne and O'Sullivan [55]) . . . . . .

2.6 Schematic of the problem of natural convection in superposed fluid and porous layers heated uniformly from below. . . . . . . . . . . . .

2.7 Streamlines (a) and isotherms (b) for natural convection in fluid superposed porous layer for $R a=10^{5}, A=4, \eta=0.5, D a=10^{-4}$, and $F=0.5$ (Poulikakos $[21]) \ldots \ldots \ldots \ldots$

2.8 Overall Nusselt number versus height ratio for convection in a fluid-superposed porous layer. (Prasad et al. [25]). . . . . . . . . . . 30

3.1 Domain of the composite two-layer problem. . . . . . . . . . . . . 35

3.2 Schematic representation of a representative elementary volume (REV) showing the different scales of analysis in porous media. . . . . . .

3.3 Schematic of (a) the computational grid, and (b) a single control volume. . . . . . . . . . . . . . . . . . . 46

3.4 Boundary control volume on an adiabatic boundary. . . . . . . . . . . 55 
4.1 Convergence characteristics of the numerical scheme. (a) Change

in average Nusselt number, $N u$ and maximum value of the stream function, $\left|\psi_{\max }\right|$ with grid refinement. (b) Percentage change

in $N u$ and $\left|\psi_{\max }\right|$ with successive grid refinement. . . . . . . . . . . . . . 64

4.2 Grid convergence of the solution in terms of the number of

iterations and the CPU time. . . . . . . . . . . . . . . 65

4.3 Energy balance given in terms of the percentage difference between the heat fluxes at the top and bottom surfaces. . . . . . . . . . 66

4.4 Time evolution of the Nusselt number the effect of the time step. . . . . . . 67

4.5 Nusselt-versus-Rayleigh number relations for the Rayleigh-Bénard problem showing comparison with literature data. . . . . . . . . . .

4.6 Nusselt-versus-Rayleigh number relations for the Horton-RogersLapwood problem. (a) Comparison to numerical studies.

(b) Comparison to experimental data. . . . . . . . . . . . . .

4.7 Nusselt-versus-Rayleigh number relations for convection in fluid-superposed porous layers. (a) Comparison with numerical results of Poulikakos [21]. (b) Comparison with numerical results of Poulikakos et al. $[20] \ldots \ldots \ldots$. . . . . . . . . . . .

4.8 Streamline and isotherm patterns for convection in a composite layer for $\delta=1$ and $\eta=0.5$. (a) $R a=10^{3}$, (b) $R a=10^{4}$, (c) $R a=10^{5}$, and (d) $R a=10^{6} \ldots \ldots \ldots \ldots$

4.9 Streamline and isotherm patterns for convection in a composite layer for $\delta=0.5$ and $\eta=0.5$. (a) $R a=10^{3}$, (b) $R a=10^{4}$, (c) $R a=10^{5}$, and (d) $R a=10^{6}$.

4.10 Streamline and isotherm patterns for convection in a composite layer for $\delta=0.25$ and $\eta=0.5$. (a) $R a=10^{3}$, (b) $R a=10^{4}$, (c) $R a=10^{5}$, and (d) $R a=10^{6}$.

4.11 Nusselt-versus-Rayleigh number relationship for different heater length ratios and $\eta=0.5 \ldots \ldots \ldots \ldots \ldots$

4.12 Dimensionless temperature $\left(T^{*}=T-T_{C} / T_{H}-T_{C}\right)$ profiles along the vertical centerline of the domain for different heater length ratios. $\eta=0.5$ and $R a=5 \times 10^{5} \ldots \ldots \ldots \ldots \ldots$

4.13 Streamline and isotherm patterns for convection in a composite layer for $\delta=0.5$ and $\eta=0.5$. (a) $R a=10^{3}$, (b) $R a=10^{4}$, (c) $R a=10^{5}$, and (d) $R a=10^{6}$.

4.14 Streamline and isotherm patterns for convection in a composite layer for $\delta=0.5$ and $\eta=0.25$. (a) $R a=10^{3}$, (b) $R a=10^{4}$, (c) $R a=10^{5}$, and (d) $R a=10^{6}$. 
4.15 Streamline and isotherm patterns for convection in a composite layer for $\delta=0.5$ and $\eta=0.75$. (a) $R a=10^{3}$, (b) $R a=10^{4}$,

(c) $R a=10^{5}$, and (d) $R a=10^{6}$.

4.16 Nusselt-versus-Rayleigh number relationship for different height

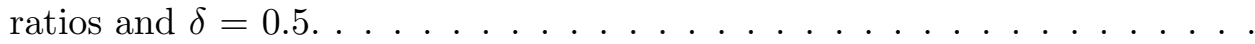

4.17 Dimensionless temperature $\left(T^{*}=T-T_{C} / T_{H}-T_{C}\right)$ profiles along the vertical centerline of the domain for different height ratios. $\delta=0.5$ and $R a=5 \times 10^{5}$.

4.18 Streamline and isotherm patterns for convection in a composite layer for $D a=10^{-6}, \delta=0.5$. (a) $R a=10^{3}$, (b) $R a=10^{4}$, (c) $R a=10^{5}$, and (d) $R a=10^{6}$.

4.19 Streamline and isotherm patterns for convection in a composite layer for $D a=10^{-4}, \delta=0.5$. (a) $R a=10^{3}$, (b) $R a=10^{4}$, (c) $R a=10^{5}$, and (d) $R a=10^{6}$.

4.20 Streamline and isotherm patterns for convection in a composite layer for $D a=10^{-2}, \delta=0.5$. (a) $R a=10^{3}$, (b) $R a=10^{4}$, (c) $R a=10^{5}$, and (d) $R a=10^{6}$.

4.21 Nusselt-versus-Rayleigh number relationship for different Darcy

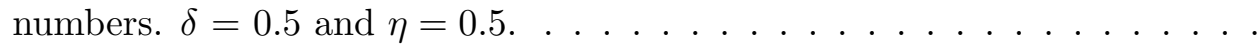

4.22 Dimensionless temperature $\left(T^{*}=T-T_{C} / T_{H}-T_{C}\right)$ profiles along the vertical centerline of the domain for different Darcy numbers. $\delta=0.5, \eta=0.5$ and $R a=5 \times 10^{5}$.

4.23 Streamline and isotherm patterns for convection in a composite layer for $A=2, \delta=0.5$. (a) $R a=10^{3}$, (b) $R a=10^{4}$, (c) $R a=10^{5}$, and (d) $R a=10^{6}$.

4.24 Streamline and isotherm patterns for convection in a composite layer for $A=4, \delta=0.5$. (a) $R a=10^{3}$, (b) $R a=10^{4}$, (c) $R a=10^{5}$, and (d) $R a=10^{6}$.

4.25 Streamline and isotherm patterns for convection in a composite layer for $A=6, \delta=0.5$. (a) $R a=10^{3}$, (b) $R a=10^{4}$, (c) $R a=10^{5}$, and (d) $R a=10^{6}$.

4.26 Streamline and isotherm patterns for convection in a composite layer for $A=6, \delta=0.75$. (a) $R a=10^{3}$, (b) $R a=10^{4}$, (c) $R a=10^{5}$, and (d) $R a=10^{6}$.

4.27 Nusselt-versus-Rayleigh number relationship for different aspect ratios. $\delta=0.5$ and $\eta=0.5 \ldots \ldots \ldots \ldots \ldots$

4.28 Dimensionless temperature $\left(T^{*}=T-T_{C} / T_{H}-T_{C}\right)$ profiles along the vertical centerline of the domain for different aspect ratios. $\delta=0.5, \eta=0.5$ and $R a=5 \times 10^{5} \ldots \ldots \ldots \ldots$ 
4.29 Streamline and isotherm patterns for convection in a composite layer for $\lambda=1, \delta=0.5$. (a) $R a=10^{3}$, (b) $R a=10^{4}$,

(c) $R a=10^{5}$, and (d) $R a=10^{6}$.

4.30 Streamline and isotherm patterns for convection in a composite layer for $\lambda=50, \delta=0.5$. (a) $R a=10^{3}$, (b) $R a=10^{4}$,

(c) $R a=10^{5}$, and (d) $R a=10^{6}$.

4.31 Streamline and isotherm patterns for convection in a composite layer for $\lambda=100, \delta=0.5$. (a) $R a=10^{3}$, (b) $R a=10^{4}$, (c) $R a=10^{5}$, and (d) $R a=10^{6}$.

4.32 Nusselt-versus-Rayleigh number relationship for different conductivity ratios. $\delta=0.5$ and $\eta=0.5 \ldots \ldots \ldots$. . . . . . .

4.33 Dimensionless temperature $\left(T^{*}=T-T_{C} / T_{H}-T_{C}\right)$ profiles along the vertical centerline of the domain for different conductivity ratios. $\delta=0.5, \eta=0.5$ and $R a=5 \times 10^{5}$.

4.34 Streamline and isotherm patterns for convection in a composite layer for $\operatorname{Pr}=7, \delta=0.5$. (a) $R a=10^{3}$, (b) $R a=10^{4}$, (c) $R a=10^{5}$, and (d) $R a=10^{6}$.

4.35 Streamline and isotherm patterns for convection in a composite layer for $\operatorname{Pr}=0.7, \delta=0.5$. (a) $R a=10^{3}$, (b) $R a=10^{4}$, (c) $R a=10^{5}$, and (d) $R a=10^{6}$.

4.36 Nusselt-versus-Rayleigh number relationship for different Prandtl numbers. $\delta=0.5$ and $\eta=0.5$

4.37 Dimensionless temperature $\left(T^{*}=T-T_{C} / T_{H}-T_{C}\right)$ profiles along the vertical centerline of the domain for different Prandtl numbers. $\delta=0.5, \eta=0.5$ and $R a=5 \times 10^{5}$.

4.38 Evolution of the flow and temperature fields in fluid-superposed porous layers heated uniformly from below. (a) $t^{*}=0$, (b) $t^{*}=1$, (c) $t^{*}=5,(\mathrm{~d}) t^{*}=10$, (e) $t^{*}=20$, (f) $t^{*}=40$, and (g) $t^{*}=60$. . $R a=10^{5}, \delta=1, \eta=0.5, \operatorname{Pr}=7, D a=10-6, A=2, \lambda=1$.

4.39 Evolution of the flow and temperature fields in fluid-superposed porous layers heated uniformly from below. (a) $t^{*}=0$, (b) $t^{*}=1$, (c) $t^{*}=5$, (d) $t^{*}=10$, (e) $t^{*}=20$, (f) $t^{*}=40$, and (g) $t^{*}=60$. . $R a=10^{5}, \delta=0.5, \eta=0.5, \operatorname{Pr}=7, D a=10-6, A=2, \lambda=1$.

5.1 Schematic of experimental setup showing the three different modules. . . . . . . . . . . . . . . . . . . .

5.2 Schematic of the convection cell showing (a) the top view and,

(b) the front view.

5.3 Schematic of the base plate assembly. (a) top view, and (b) side view. . . . . . . . . . . . . . . . . . . 
5.4 Schematic diagram of the top plate assembly showing the different components. . . . . . . . . . . . . . . .

5.5 Thermocouple locations at (a) main heater surface, (b) top plate, and (c) guard heater surface. . . . . . . . . . . . . . . . . . 126

5.6 (a) Position of the thermocouple frame inside the cavity.

(b) Thermocouple locations on the frame. . . . . . . . . . . . . . . . . 127

5.7 Power supply circuit to the main and guard heaters. . . . . . . . . . . 128

5.8 Overall experimental setup. . . . . . . . . . . . . . . . . . . . . 129

6.1 Thermal resistance network for the side wall. . . . . . . . . . . . . . . 134

6.2 Finite-element simulation of the heat transfer problem in the base plate. (a) Problem setup and boundary conditions, (b) finite-element grid used, (c) steady-state temperature, contours and $(\mathrm{d})$ heat flow lines. . . . . . . . . . . . . . . . 135

6.3 Thermal resistance network for the base plate. . . . . . . . . . . . . 136

6.4 Comparison of current experimental data with the numerical and experimental results of Rajen and Kulacki [60]. . . . . . . . . . . . . 139

6.5 Comparison of current experimental results with the numerical and experimental results of Lai and Kulacki [61]. . . . . . . . . . . . . . . . 139

6.6 Nusselt-versus-Rayleigh number relation for $\eta=0.5, \delta=0.44$.

Error bars indicate the total uncertainties. . . . . . . . . . . . . . . . . 143

6.7 Nusselt-versus-Rayleigh number relation for $\eta=0.67, \delta=0.44$. Error bars indicate the total uncertainties. . . . . . . . . . . . . . . 143

6.8 Nusselt-versus-Rayleigh number relation for $\eta=0.75, \delta=0.44$. Error bars indicate the total uncertainties. . . . . . . . . . . . . . . . . . 144

6.9 Nusselt-versus-Rayleigh number relation for $\eta=0.5, \delta=0.11$.

Error bars indicate the total uncertainties. . . . . . . . . . . . . . . . . . 144

6.10 Effect of height ratio, $\eta$, on the overall Nusselt-versus-Rayleigh number relations $(\delta=0.5) \ldots \ldots \ldots \ldots \ldots \ldots \ldots$

6.11 Effect of heater length ratio, $\delta$, on the overall Nusselt-versusRayleigh number relations $(\eta=0.5) \ldots \ldots \ldots \ldots$

6.12 Non-dimensional temperature profiles along the length of the convection cell and along the horizontal mid-plane at three different $R a$ values. The position of the heater is also shown on the $x$-axis. . . . . . . . . . . . . . . . . . 147

6.13 Non-dimensional temperature profiles along the depth of the convection cell and along the horizontal mid-plane at three different $R a$ values. . . . . . . . . . . . . . . . . . . . . 147 
7.1 Comparison of numerical and experimental data for $\delta=0.44$,

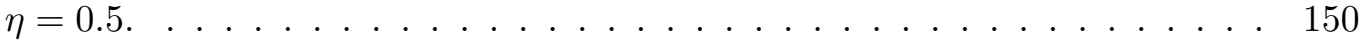

7.2 Comparison of numerical and experimental data for $\delta=0.44$,

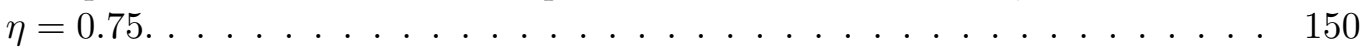

7.3 Comparison of the numerical and experimental results of Prasad and Kulacki [58], and Rajen and Kulacki [60]. . . . . . . . . . . . . 154

7.4 Comparison of simulations results for $\delta=1$ and $\eta=0.5,0.8$ with the experimental results of Prasad and Tian [24] . . . . . . . . . . . 155

7.5 Comparison of present results for $\delta=1$ with all published numerical studies. . . . . . . . . . . . . . . . . . . . 158

7.6 Comparison of present experimental results with all existing experiment studies. . . . . . . . . . . . . . . . . . 161 


\section{Nomenclature}

\begin{tabular}{|c|c|}
\hline$A$ & aspect ratio, $L / H$ \\
\hline$A_{1}$ & aspect ratio, $H / L$ \\
\hline$A_{\text {tot }}$ & total area of the heaters, $\mathrm{m}^{2}$ \\
\hline$A_{\text {base }}$ & area of the base plate, $\mathrm{m}^{2}$ \\
\hline$A_{\text {wall }}$ & area of the side wall, $\mathrm{m}^{2}$ \\
\hline$A_{\text {ins }}$ & area of the insulation, $\mathrm{m}^{2}$ \\
\hline$a$ & grid point coefficient, $\mathrm{m}^{2} / \mathrm{s}$ \\
\hline$a_{P}^{0}$ & grid point time constant, $\mathrm{m}^{2} / \mathrm{s}$ \\
\hline$b$ & right hand side of discretization equation \\
\hline$C$ & grid spacing coefficient \\
\hline$c_{K}$ & Kozeny constant \\
\hline$c_{p}$ & heat capacity, $\mathrm{J} / \mathrm{kg} \mathrm{K}$ \\
\hline$D$ & grid point parameter, $\mathrm{m}^{2} / \mathrm{s}$ \\
\hline$d$ & particle diameter, $\mathrm{m}$ \\
\hline$d_{p}$ & pore diameter, $\mathrm{m}$ \\
\hline$D a$ & Darcy number, $K / H^{2}$ \\
\hline$D a_{p}$ & Darcy number based on the particle diameter, $K / d^{2}$ \\
\hline$F$ & Forchheimer coefficient, $1.75 /\left(175^{1 / 2} \phi^{1 / 3}\right)$ \\
\hline$g$ & acceleration due to gravity, $\mathrm{m}^{2} / \mathrm{s}$ \\
\hline$G r_{\mathrm{m}}$ & porous media Grashof number, $g \beta H^{3}\left(T_{H}-T_{C}\right) / \nu^{2}$ \\
\hline$H$ & total height of the problem domain, $m$ \\
\hline$h$ & average heat transfer coefficient, $\mathrm{W} / \mathrm{m}^{2}$ \\
\hline$H_{1}$ & height of bottom porous layer in a two-layer system, m \\
\hline$H_{2}$ & height of top porous layer in a two-layer system, $\mathrm{m}$ \\
\hline
\end{tabular}




\begin{tabular}{|c|c|}
\hline$H_{\text {base }}$ & thickness of the base plate, $\mathrm{m}$ \\
\hline$H_{\mathrm{f}}$ & height of the fluid layer, $m$ \\
\hline$H_{\mathrm{m}}$ & height of the porous layer, $m$ \\
\hline$k_{1}$ & thermal conductivity of bottom porous layer in a two-layer system, $\mathrm{W} / \mathrm{m} \mathrm{K}$ \\
\hline$k_{2}$ & thermal conductivity of top porous layer in a two-layer system, W/m K \\
\hline$k_{\text {base }}$ & thermal conductivity of the base plate, $\mathrm{W} / \mathrm{m} \mathrm{K}$ \\
\hline$k_{\text {eff }}$ & effective thermal conductivity of Prasad et al. [43], W/m K \\
\hline$k_{\mathrm{f}}$ & thermal conductivity of the saturating fluid, $\mathrm{W} / \mathrm{m} \mathrm{K}$ \\
\hline$k_{\text {ins }}$ & thermal conductivity of the insulation, $\mathrm{W} / \mathrm{m} \mathrm{K}$ \\
\hline$k_{\mathrm{SO}}$ & thermal conductivity of the solid matrix, $\mathrm{W} / \mathrm{m} \mathrm{K}$ \\
\hline$k_{\mathrm{m}}$ & stagnant thermal conductivity of the porous layer, $\mathrm{W} / \mathrm{m} \mathrm{K}$ \\
\hline$k_{\text {wall }}$ & thermal conductivity of the side wall, $\mathrm{W} / \mathrm{m} \mathrm{K}$ \\
\hline$J$ & flow rate across control volume face, $\mathrm{m}^{2} / \mathrm{s}$ \\
\hline$K$ & permeability of the porous medium, $\mathrm{m}^{2}$ \\
\hline$K_{1}$ & permeability of the bottom porous layer in a two-layer system \\
\hline$K_{2}$ & permeability of the top porous layer in a two-layer system \\
\hline$K C$ & Kozeny-Carmen number, $c_{K} H / K$ \\
\hline$L$ & length of the problem domain, $m$ \\
\hline$M$ & coefficient matrix for the discretized equations \\
\hline$N$ & total number of grid points \\
\hline$n_{H}$ & number of thermocouples on heater surface \\
\hline$n_{C}$ & number of thermocouples on the top surface \\
\hline$N u$ & Nusselt number based on the fluid conductivity, $h H / k_{\mathrm{f}}$ \\
\hline$N u_{\mathrm{m}}$ & Nusselt number based on the stagnant conductivity, $h H / k_{\mathrm{m}}$ \\
\hline$N u_{B}$ & Nusselt number along the bottom surface of the problem domain \\
\hline$N u_{T}$ & Nusselt number along the top surface of the problem domain \\
\hline$q$ & heat energy supplied by the heaters, W \\
\hline$q_{\text {loss }}$ & total heat loss, W \\
\hline$q_{\mathrm{lb}}$ & heat loss through the base, $\mathrm{W}$ \\
\hline$q_{\mathrm{ls}}$ & heat loss through a single side wall, W \\
\hline$q_{\text {side }}$ & total heat loss through the side walls, W \\
\hline$q^{\prime \prime}$ & heat flux produced by the heaters, $\mathrm{W} / \mathrm{m}^{2}$ \\
\hline
\end{tabular}




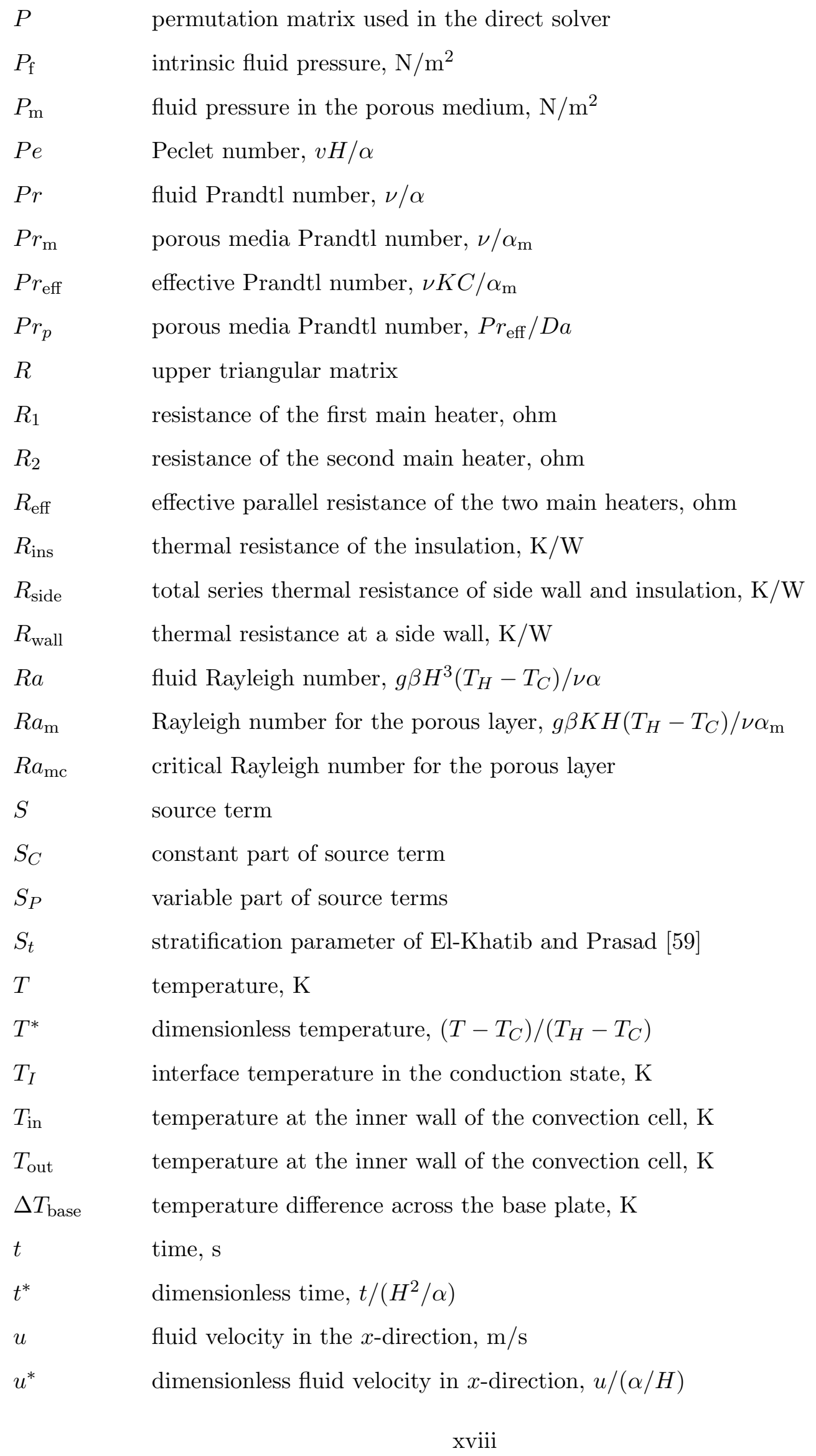




\begin{tabular}{|c|c|}
\hline$U_{A_{\text {tot }}}$ & uncertainty in $A_{\text {tot }}, \mathrm{m}^{2}$ \\
\hline$U_{c_{p}}$ & uncertainty in $c_{p}, \mathrm{~J} / \mathrm{kg} \mathrm{K}$ \\
\hline$U_{H}$ & uncertainty in $H, \mathrm{~m}$ \\
\hline$U_{k_{\mathrm{f}}}$ & uncertainty in $k_{\mathrm{f}}, \mathrm{W} / \mathrm{m} \mathrm{K}$ \\
\hline$U_{L_{H}}$ & uncertainty in $L_{H}, \mathrm{~m}$ \\
\hline$U_{N u}$ & uncertainty in $N u$ \\
\hline$U_{q_{\text {net }}}$ & uncertainty in $q_{\text {net }}, \mathrm{W}$ \\
\hline$U_{R a}$ & uncertainty in $R a$ \\
\hline$U_{T}$ & uncertainty in $T, \mathrm{~K}$ \\
\hline$U_{T_{C}}$ & uncertainty in the average top surface temperature, $\mathrm{K}$ \\
\hline$U_{T_{H}}$ & uncertainty in the average heater surface temperature, $\mathrm{K}$ \\
\hline$U_{W_{H}}$ & uncertainty in $W_{H}, \mathrm{~m}$ \\
\hline$U_{\alpha}$ & uncertainty in $\alpha, \mathrm{m}^{2} / \mathrm{s}$ \\
\hline$U_{\beta}$ & uncertainty in $\beta, 1 / \mathrm{K}$ \\
\hline$U_{\Delta T}$ & uncertainty in $\Delta T, \mathrm{~K}$ \\
\hline$U_{\mu}$ & uncertainty in $\mu, \mathrm{kg} / \mathrm{m} \mathrm{s}$ \\
\hline$U_{\nu}$ & uncertainty in $\nu, \mathrm{m}^{2} / \mathrm{s}$ \\
\hline$U_{\rho}$ & uncertainty in $\rho, \mathrm{kg} / \mathrm{m}^{3}$ \\
\hline$V$ & voltage drop across the main heaters, $\mathrm{V}$ \\
\hline$v$ & fluid velocity in the $y$-direction, $\mathrm{m} / \mathrm{s}$ \\
\hline$v^{*}$ & dimensionless fluid velocity in $y$-direction, $v /(\alpha / H)$ \\
\hline$v_{t}$ & vertical throughflow velocity of Chen $[11], \mathrm{m} / \mathrm{s}$ \\
\hline$W_{H}$ & width of the heaters, $\mathrm{m}$ \\
\hline$w_{\text {ins }}$ & width of the insulation, $\mathrm{m}$ \\
\hline$w_{\text {wall }}$ & width of the side wall, $\mathrm{m}$ \\
\hline$x, y, z$ & Cartesian co-ordinates, m \\
\hline$x^{*}, y^{*}, z^{*}$ & dimensionless co-ordinates, $x / H, y / H, z / H$ \\
\hline
\end{tabular}

\section{Greek letters}

$\alpha \quad$ thermal diffusivity, $\mathrm{m}^{2} / \mathrm{s}$

$\hat{\alpha} \quad$ Beavers-Joseph constant

$\beta \quad$ coefficient of thermal expansion of the fluid, $1 / \mathrm{K}$ 


\begin{tabular}{|c|c|}
\hline$\Gamma$ & generic diffusion coefficient, $\mathrm{m}^{2} / \mathrm{s}$ \\
\hline$\gamma$ & non-dimensional particle diameter, $d / H$ \\
\hline$\Delta x$ & length of control volume, $\mathrm{m}$ \\
\hline$\Delta y$ & width of control volume, $\mathrm{m}$ \\
\hline$\delta$ & heater-to-base length ratio, $L_{\mathrm{H}} / L$ \\
\hline$\delta_{1}$ & heater-to-total height length ratio, $L_{\mathrm{H}} / H$ \\
\hline$\delta x$ & grid spacing in $x$-direction, $\mathrm{m}$ \\
\hline$\delta y$ & grid spacing in $y$-direction, $\mathrm{m}$ \\
\hline$\epsilon$ & thermal diffusivity ratio, $\alpha_{\mathrm{f}} / \alpha_{\mathrm{so}}$ \\
\hline$\eta$ & ratio of porous layer height to total height, $H_{\mathrm{m}} / H$ \\
\hline$\eta_{1}$ & ratio of porous layer height to fluid layer height, $H_{\mathrm{m}} / H_{\mathrm{f}}$ \\
\hline$\zeta$ & vertical throughflow strength of Chen $[11], v_{t} H_{\mathrm{m}} / \alpha_{\mathrm{m}}$ \\
\hline$\Theta$ & vector of unknowns in discretization equation \\
\hline$\theta$ & generic diffusion variable $(T$ or $\omega)$ \\
\hline$\kappa$ & effective thermal conductivity ratio, $k_{\mathrm{m}} / k_{\mathrm{f}}$ \\
\hline$\Lambda$ & viscosity ratio, $\mu_{\mathrm{eff}} / \mu_{\mathrm{f}}$ \\
\hline$\lambda$ & thermal conductivity ratio, $k_{\mathrm{so}} / k_{\mathrm{f}}$ \\
\hline$\mu$ & dynamic viscosity of saturating fluid, N-s $/ \mathrm{m}^{2}$ \\
\hline$\nu$ & kinematic viscosity of saturating fluid, \\
\hline$\Xi$ & viscosity variation parameter of Chen and $\mathrm{Wu}[14]$ \\
\hline$\xi$ & horizontal to vertical permeability ratio in an anisotropic porous layer \\
\hline$\varpi$ & weighting factor of Prasad et al. [44] \\
\hline$\rho$ & density, $\mathrm{kg} / \mathrm{m}^{3}$ \\
\hline$\sigma$ & effective heat capacity ratio, $\left(\rho c_{p}\right)_{\mathrm{m}} /\left(\rho c_{p}\right)_{\mathrm{f}}$ \\
\hline$\phi$ & porosity the porous layer \\
\hline$\chi$ & horizontal to vertical thermal diffusivity ratio in an anisotropic porous layer \\
\hline$\psi$ & non-dimensional stream function \\
\hline$\Omega$ & relaxation parameter \\
\hline$\omega$ & non-dimensional vorticity \\
\hline
\end{tabular}

\section{Subscripts}

reference state 


\begin{tabular}{|c|c|}
\hline 1 & first heater \\
\hline 2 & second heater \\
\hline$\infty$ & ambient properties \\
\hline avg & average/mean value \\
\hline $\mathrm{B}$ & boundary grid point \\
\hline $\mathrm{b}$ & bottom surface \\
\hline base & base plate \\
\hline $\mathrm{C}$ & cooled wall \\
\hline $\mathrm{c}$ & critical value \\
\hline $\mathrm{cl}$ & centerline of the heaters \\
\hline cs & cross section of base plate \\
\hline $\mathrm{CN}$ & constant \\
\hline $\mathrm{E}$ & east grid point \\
\hline e & east face of control volume \\
\hline eff & effective properties \\
\hline $\mathrm{f}$ & fluid properties \\
\hline $\mathrm{H}$ & heated wall \\
\hline $\mathrm{i}$ & index \\
\hline in & inner wall of apparatus \\
\hline ins & insulation properties \\
\hline int & interface properties \\
\hline $\mathrm{j}$ & index \\
\hline loss & total loss \\
\hline 1 & total loss through the side walls \\
\hline $\mathrm{lb}$ & loss through base plate \\
\hline ls & loss through a single side wall \\
\hline $\mathrm{m}$ & porous layer properties \\
\hline $\mathrm{N}$ & north grid point \\
\hline $\mathrm{n}$ & north face of control volume \\
\hline ne & control volume north-east corner point \\
\hline net & net value \\
\hline nw & control volume north-west corner point \\
\hline
\end{tabular}




$\begin{array}{ll}\text { out } & \text { outside wall of apparatus } \\ \text { P } & \text { control volume grid point } \\ \text { S } & \text { south grid point } \\ \text { s } & \text { south fact of control volume } \\ \text { se } & \text { control volume south-east corner point } \\ \text { so } & \text { solid properties } \\ \text { Sw } & \text { control volume south-west corner point } \\ \text { side } & \text { side wall of apparatus } \\ \text { t } & \text { top surface } \\ \text { tot } & \text { total } \\ \text { W } & \text { west grid point } \\ \text { w } & \text { west control volume face }\end{array}$

\section{Superscripts}

$0 \quad$ value during previous time step

$1 \quad$ value during current time step

$+\quad$ positive $x$ or $y$ direction

- $\quad$ negative $x$ or $y$ direction

* dimensionless parameter 


\section{Chapter 1}

\section{Introduction}

\section{$1.1 \quad$ Background}

A porous medium can loosely be defined as a solid with an interconnected void space that is filled with one or more fluid phases [1]. Figure 1(a) shows a schematic diagram of an idealized porous medium that is comprised of uniform spherical particles saturated with a single fluid. In general, however, porous media, especially naturally occurring ones, have an irregular geometry as shown in Figure 1(b). Porous media are ubiquitous in nature and can also be found in several engineering applications. Examples of naturally occurring porous media include beach sand, sandstone, limestone, rye bread, wood, and the human lung, while engineering applications involving porous media include packed bed reactors, geothermal energy extraction, energy storage devices, and thermal insulation systems.

Due to the wide range of situations in which porous media are encountered, the study of heat transfer in these systems has received great deal of attention from both the scientific and engineering communities. One particular problem that has attracted significant attention is that of natural convection in horizontal porous layers uniformly heated from below. Beginning with the pioneering studies of Horton and Rogers [2] and Lapwood [3], this problem, which constitutes the porous media equivalent of the Rayleigh-Bénard problem for pure fluids, has been studied extensively over the last fifty years. Several variations of this problem have also been studied in great detail. These include convection in multi-layered porous media, convection with localized heat sources, and convection in anisotropic porous media. These studies have been primarily motivated by practical applications, such as the disposal of high level nuclear wastes in deep geological repositories,

cooling of electronic devices, design of energy efficient buildings, and geothermal energy extraction. This problem is also important from a fundamental scientific perspective as it an example of a system where a well-defined flow structure develops from an initial random 
(a)

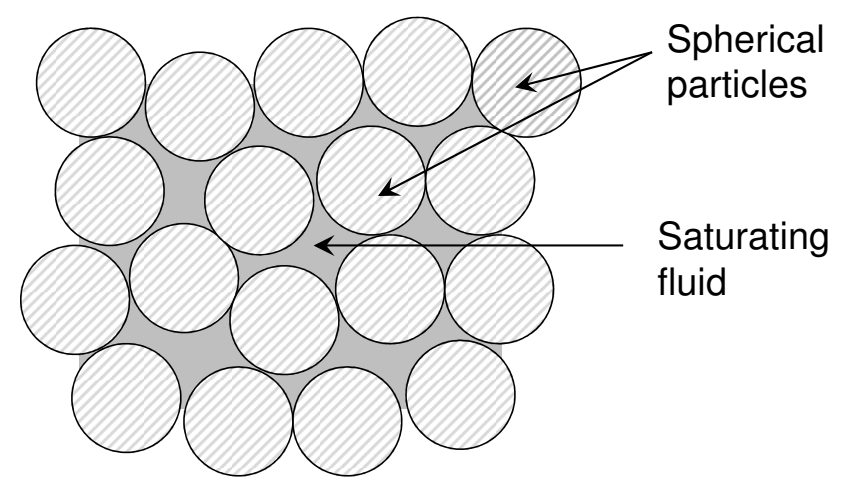

(b)

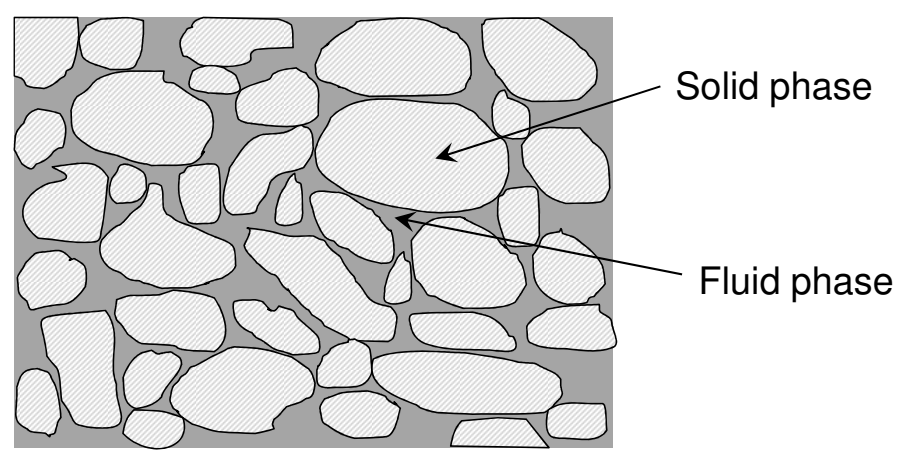

Figure 1.1: Schematic diagram of (a) an idealized saturated porous medium, and (b) a naturally occurring porous media.

disturbance and therefore allows for a fundamental investigation of stability modes. Additionally, the mathematical formulation of the problem is one of the simplest non-linear elliptic systems. As a result, it has been an ideal test problem for numerous numerical studies. Indeed, the amount of literature available on this topic is a measure alone of its scientific importance. Excellent review articles by Combarnous and Bories [4] and Cheng [5] give detailed accounts of the early advances in this field while, more recently, books by Neild and Bejan [1] and Kaviany [6] give a comprehensive overview of the current state of research in this area.

An important variation of this problem is that of natural convection in a horizontal composite system that consists of a fluid layer overlying a porous layer saturated with the same fluid. This problem is of great importance from a geophysical perspective. Thermal circulation in lakes and shallow coastal areas, flows in geysers and hot springs, and solutal convection in sub-soil water reservoirs are all examples of this problem. This problem also arises in several engineering and industrial applications such as fibrous and granular thermal insulations, water reservoirs, grain storage installations, solid-matrix heat exchangers, solidifying castings, and post-accident cooling of nuclear reactors. 
In spite of this universality, however, this problem has not been investigated in great depth. Most existing studies on this topic deal with the problem of onset of convection in composite system heated uniformly from below [7-19]. These studies have identified several important aspects of this problem, most importantly the fact that convection in such a composite system has a bimodal character and that the wavelength of the convective mode at the onset point depends on the fluid-to-porous layer height ratio [9]. Even then, existing studies do not agree on an exact critical point. This is primarily due to the fact that there is no agreement on the most accurate approach to modeling fluid motion in this system. In particular, there has been extensive debate on the appropriate form of the boundary conditions at the porous-fluid layer interface. Three different modeling approaches have been proposed and all of them predict somewhat different critical onset points. In recent years, there has been much work [15-19] in reconciling the different models and identifying reasons for the observed discrepancy of the results. However, the lack of conclusive experimental studies has led to the problem being, as yet, unresolved.

Similarly, the overall heat transfer characteristics of the system at high Rayleigh numbers are not very well understood. Only a handful of studies have explored this problem from a theoretical [20-23] perspective, and they report widely different results. This can, in part, be attributed directly to the different modeling approaches adopted in these studies. A comprehensive set experimental studies by Prasad and co-workers [24-26] are available but they have not been confirmed by other investigators. Also, no direct comparison of experimental and theoretical studies at high Rayleigh numbers has been published which has further compounded the validation of the numerical studies.

Another significant shortcoming of the literature on the dual-layer convection problem is that almost all existing studies consider the case where the composite layer is uniformly heated from below. A uniformly heated bottom, however, is most often an idealization that is rarely realized in nature where, many a times, the heat source is a local or concentrated one. This is especially true in geophysical environments where troughs of volcanic debris act as localized heat sources. In addition, many practical situations involving fluid-superposed layers deal with localized heat sources. It has been shown that in porous layers heated locally from below, the evolution of the temperature and flow fields, and also the overall heat transfer coefficients, are markedly influenced by the size of the heat source. As such, it is vital to examine the problem of natural convection in fluid-superposed porous layers heated locally from below. However, an extensive search of the literature of the last thirty years has yielded minimal information pertaining to this particular problem. This is a major shortcoming of the current state of knowledge of this topic which needs to be addressed in order to obtain a complete understanding of the problem and its various 
aspects. To meet this goal, a thorough investigation of the problem of convection in fluidsuperposed porous layers is undertaken in this thesis with an emphasis on the particular case of a localized bottom heat source.

\subsection{Aims and objectives of the thesis}

The primary aim of this thesis is to present a study of natural convection in horizontal fluid-superposed porous layers heated locally from below. The principal objective is to make a fundamental contribution to the existing literature on convection in porous layers. As this problem has not been studied previously, this thesis will add to prior studies on this topic and thus help to put together a complete overall picture of this problem. In order to meet these objectives, it is important to understand how the presence of a localized heat source at the base of a fluid-superposed porous layer causes flow instability and influences the evolution of the flow and the temperature fields. It is also desirable to understand the heat transfer characteristics of this system and its dependence on various parameters such as the size of the heat source, the fluid layer-to-porous layer height ratio, the particle size, the aspect ratio of the problem domain, the solid-to-fluid conductivity ratio, and the fluid Prandtl number. Therefore, the bulk of the present work is aimed at studying, in detail, the effects of the aforementioned parameters across a wide range of Rayleigh numbers with particular emphasis on the high Rayleigh number range that is important for most engineering applications.

A complete study of the effects of each individual parameter on the overall heat transfer rate and flow and temperature fields is however a formidable task. In order to systematically analyze the problem, it is studied from both a theoretical and experimental perspective. These two approaches are adopted for separate purposes. The theoretical component of the study focuses on numerically solving the governing equations of mass, momentum and energy conservation in order to understand the steady-state velocity and temperature fields, as well as to predict of the overall heat transfer rates. The advantage of numerical simulation is that the effects of each of the independent controlling parameters can be studied separately in detail to yield a comprehensive set of results for all the cases. An experimental study of comparable breadth would require enormous resources and is thus not feasible. Additionally, accurate experimental data is difficult to obtain for low Rayleigh numbers because of the high levels of uncertainty in the measurements. However, there are some limitations inherent in the numerical solutions which have to be addressed with experimental studies. First, the accuracy of the numerical results is open to question in the light of the fact that there is yet no universally accepted mathematical model for fluid flow in a dual-layer system. In addition, the highly non-linear nature of the governing equations 
at high Rayleigh numbers makes it computationally feasible to obtain numerical solutions only up to moderately high Rayleigh numbers; at higher Rayleigh numbers, computational costs can be prohibitive.

These drawbacks can be addressed by the experimental component of the study. A carefully designed set of experiments is sufficient to determine the validity of the numerical model. Additionally, these experiments can help in conclusively determining the most appropriate mathematical model for natural convection in a fluid-superposed porous layer system. Also, as opposed to the numerical simulations, it is much easier to obtain measurements of heat transfer rates at high Rayleigh numbers. Thus measurements will be made at high Rayleigh numbers for the most important parameters. A combination of numerical and experimental results can then be used to create a data set of heat transfer rates over a wide range of Rayleigh numbers for the most important controlling parameters. Therefore a combination of numerical and experimental studies will be necessary to meet the aims of this thesis.

\subsection{Structure and organization of the thesis}

In the next chapter, the literature relevant to the problem at hand is reviewed in detail with a particular emphasis on studies published over the last twenty five years. In particular, studies on convection in porous layers with uniform and localized heat sources, and in fluid-superposed porous layers heated uniformly are reviewed. The mathematical model and the numerical solution technique are discussed in detail in the following chapter. Chapter 4 discusses the numerical results in detail. Subsequently the design of the experimental setup is discussed. Chapter 6 gives the experimental results along with a comparison with selected numerical results. Finally the conclusions of the thesis are given along with recommendations for future work. 


\section{Chapter 2}

\section{Literature Review}

As mentioned earlier, the literature pertaining to the problem being studied in this thesis is very limited. However there is an extensive amount of literature on natural convection in porous and fluid-superposed porous layers. These studies are of fundamental importance as they identify the salient parameters that affect convection heat transfer in porous media. A thorough review of the relevant literature will thus provide the framework within which a coherent definition for the current problem can be constructed. To that end, the literature on convection in porous layers heated uniformly from below is reviewed first. Thereafter, the literature on porous media convection with localized bottom heat sources is studied. Finally, studies on convection in fluid-superposed porous layers heated uniformly from below are discussed. Based on these studies, it will be possible to identify the factors that are most likely to play a significant role in the present study.

\subsection{Natural convection in horizontal porous layers heated uniformly}

The problem of natural convection in a horizontal porous medium heated from below can be schematically represented as shown in Figure 2.1. This problem was first studied over fifty years ago by Horton and Rogers [2] with the aim of understanding the movement of convection currents in deep geological layers. Performing a linear stability analysis, they found that the convection occurs when $R a_{\mathrm{m}}=4 \pi^{2}$, where $R a_{\mathrm{m}}$ is the porous medium Rayleigh number. This problem was studied independently by Lapwood [3], who obtained an identical condition for the onset of convection. Since then, an extensive amount of work examining the various aspects of this problem has been done. As such, it would not be possible to give an exhaustive literature review of the subject. Instead, the literature review will focus on studies which are relevant to the problem at hand and can provide crucial insights into the fundamental aspects of the problem. Of particular importance are the experimental studies which measure the heat transfer characteristics of natural 


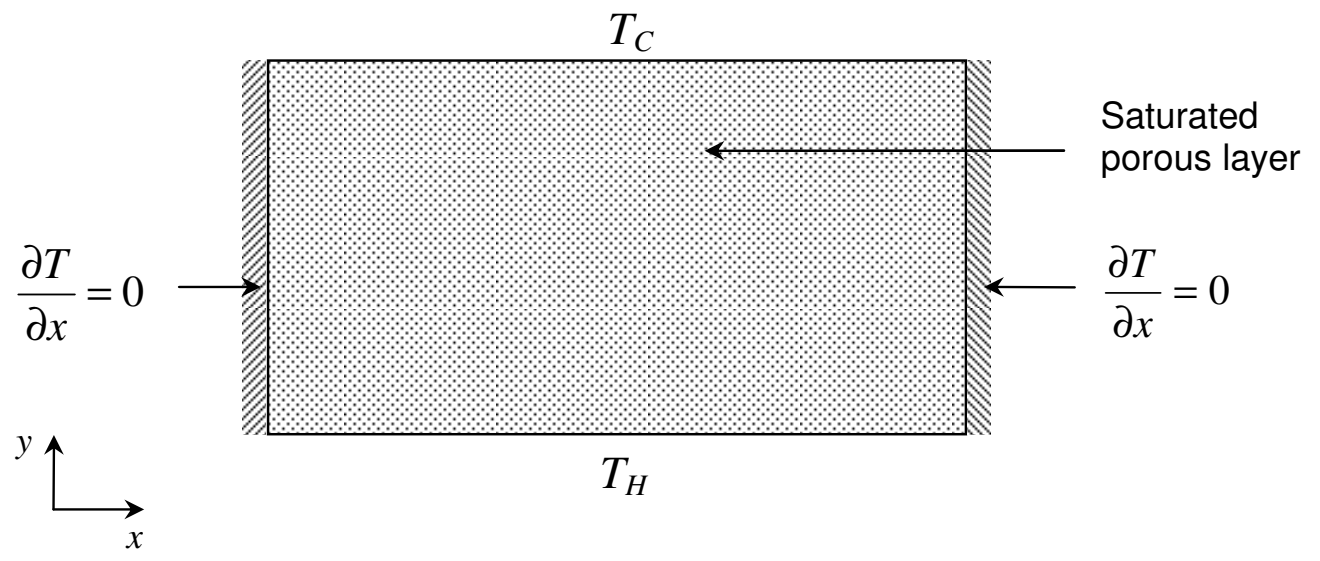

Figure 2.1: Schematic of the basic problem of natural convection in a horizontal porous layer heated uniformly from below.

convection in a porous layer.

A large number of experimental studies on natural convection in horizontal porous layers heated uniformly from below are available in the literature. Figure 2.2 shows a compilation of the primary experimental data for this problem [27]. The principal aim of experimental studies in this area has been to obtain a relationship of the form $N u_{\mathrm{m}}=$ $C \times R a_{\mathrm{m}}^{n}$, where $C$ and $n$ are constants, and the Nusselt number, $N u_{\mathrm{m}}$, and Rayleigh number, $R a_{\mathrm{m}}$, are,

$$
\begin{gathered}
N u_{\mathrm{m}}=\frac{h H}{k_{\mathrm{m}}}, \\
R a_{\mathrm{m}}=\frac{g \beta K H \Delta T}{\nu \alpha_{\mathrm{m}}} .
\end{gathered}
$$

Here, $k_{\mathrm{m}}$ and $\alpha_{\mathrm{m}}$ are the effective stagnant conductivity, and the diffusivity of the porous medium respectively and are,

$$
\begin{gathered}
k_{\mathrm{m}}=\phi k_{\mathrm{f}}+(1-\phi) k_{\mathrm{m}}, \\
\alpha_{\mathrm{m}}=\frac{k_{\mathrm{m}}}{\left(\rho c_{\mathrm{p}}\right)_{\mathrm{m}}}
\end{gathered}
$$

where $\phi$ is the porosity of the porous medium, and $\left(\rho c_{\mathrm{p}}\right)_{\mathrm{f}}$ is the heat capacity of the saturating fluid. 


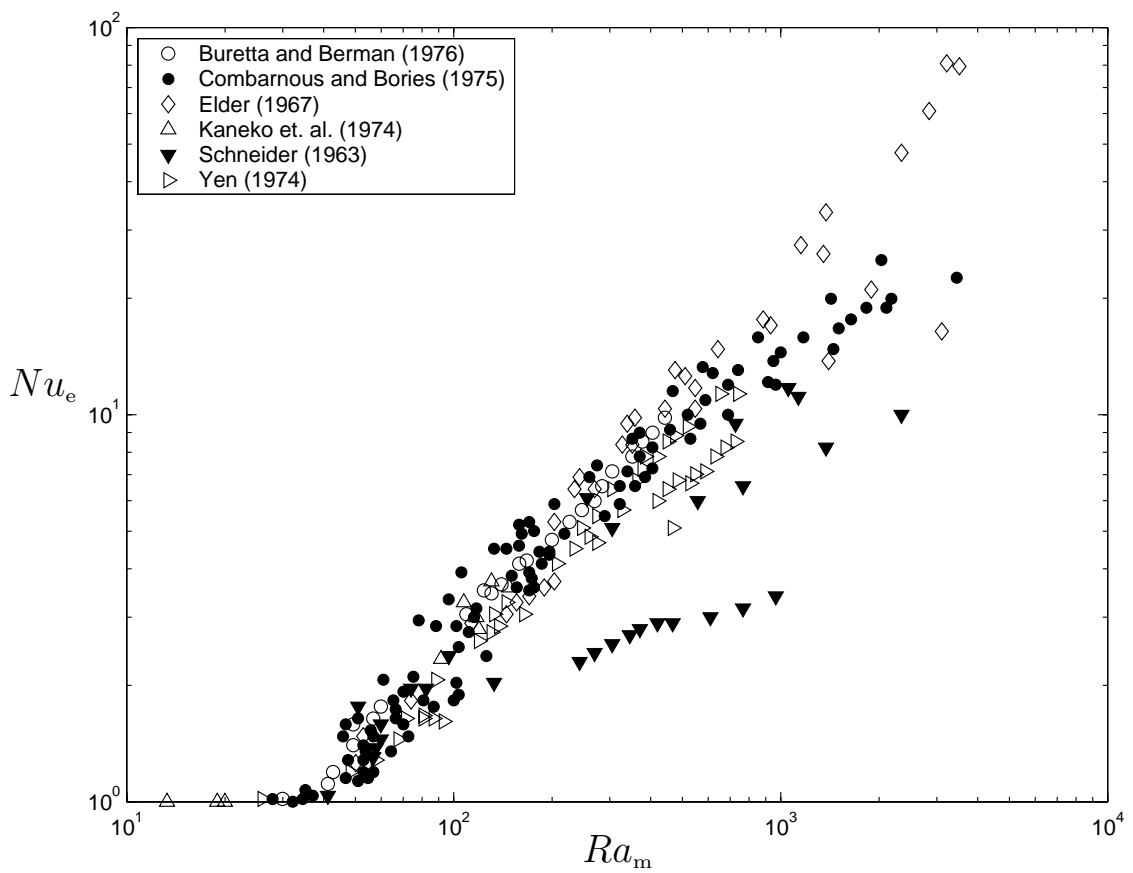

Figure 2.2: Compilation of experimental results of Nusselt number versus Rayleigh number for convection in a horizontal porous layer heated uniformly from below (Aichlmayr [27]).

Schneider [28] was the first to report experimental studies on free convection in a horizontal porous layer. He conducted experiments with a wide range of porous media and saturating fluids and found that for a given Rayleigh number there is a large variation in the Nusselt number for steel-oil, glass-water and glass-oil systems. This divergence is particularly pronounced at high Rayleigh numbers as can be seen in Figure 2.2. Schneider noted that the data can be roughly correlated at low Rayleigh numbers if the Nusselt number is based upon the effective stagnant conductivity of the saturated porous layer, $k_{\mathrm{m}}$. To correlate the data at relatively higher values of $R a_{\mathrm{m}}$, he proposed to base the Nusselt number upon the fluid thermal conductivity, $k_{\mathrm{f}}$, rather than $k_{\mathrm{m}}$, and found that this change reduced the scatter in the data. Schneider argued that when $R a_{\mathrm{m}}$ is large, the fluid thermal conductivity somehow exerts a greater influence on the heat transfer than the solid phase, and hence it is more appropriate to base the Nusselt number on $k_{\mathrm{f}}$.

Elder [29] also conducted experimental studies on natural convection in a horizontal porous layer heated uniformly from below. For his experiments he used a cylindrical test section with 3, 5, 8 and $18 \mathrm{~mm}$ DIA glass spheres, and expanded $6 \mathrm{~mm}$ DIA Styropor ${ }^{\circledR}$ balls as the porous layer. Distilled water was used as the saturating fluid. Elder found that around $R a_{\mathrm{m}} \sim 40$, there is an abrupt change in the value of the Nusselt number indicating 
that the onset of convection occurs when the Rayleigh number reaches the theoretically predicted critical value of $4 \pi^{2}$. Beyond this critical value, for low values of the Rayleigh number, he found that the data could be well correlated by the relation $N u_{\mathrm{m}}=R a_{\mathrm{m}} / 40 \pm$ $10 \%$. At higher values of $R a_{\mathrm{m}}$, however, he found that this is no longer possible. Elder noted that the departure of the data from this correlation closely follows the relationship $N u_{\mathrm{m}} \sim R a_{\mathrm{m}}^{1 / 4}$, which is valid for simple viscous convection. He therefore concluded that it is more appropriate to use the fluid Nusselt and Rayleigh numbers, $N u$ and $R a$, to correlate the data in the high Rayleigh number regime. He showed that correlating the data with $N u$ and $R a$ yielded relations that were remarkably similar to those reported in the literature for viscous laminar convection between horizontal planes.

Katto and Masuoka [30] performed experiments with a wide range of porous media to ascertain the criterion for the onset of convection. Their experiments were conducted in a cylindrical test section with glass, steel and aluminium spheres of different sizes as the porous material. To reduce the temperature difference between the upper and lower surfaces required for creating large Rayleigh numbers, they used compressed nitrogen gas as the saturating fluid. The main objective of the experiments was to determine whether parameters such as the size of the porous material, the porosity of the layer, and the solid-tofluid conductivity ratio affect the critical Rayleigh number. They found that the condition $R a_{\mathrm{m}}=4 \pi^{2}$ is remarkably accurate over a wide range of porous media provided the thermal diffusivity used in the definition of the Rayleigh number is given by equation (2.4).

In a subsequent study, Masuoka [31] performed experiments to determine the relationship between the Nusselt and the Rayleigh numbers. His experiments used 1.85 and $3.12 \mathrm{~mm}$ DIA glass beads as the porous layer and distilled water as the saturating fluid. The height of the porous layer was varied between $30 \mathrm{~mm}$ and $100 \mathrm{~mm}$. Masuoka found that near the critical Rayleigh number of 40, the Nusselt-versus-Rayleigh number relation can be expressed as $N u_{\mathrm{m}}=1+2\left(1-4 \pi^{2}\right) / R a_{\mathrm{m}}$. This relationship is valid up to $R a_{\mathrm{m}}=2 R a_{\mathrm{mc}}$, where $R a_{\mathrm{mc}}$ is the critical Rayleigh number for the onset of convection. Beyond $2 R a_{\mathrm{mc}}$ he found that the Nusselt number is independent of the layer height and the effective thermal conductivity of the porous medium, $k_{\mathrm{m}}$, and is directly proportional to $R a_{\mathrm{m}}$.

Combarnous and others [32-34] performed several experimental studies on natural convection in porous layers which are reported in Combarnous and Bories [4]. In these studies, polypropylene (4 mm DIA), glass (0.9, 2, 3, 4, $1.7 \mathrm{~mm}$ DIA), quartz $(1.9,2.25 \mathrm{~mm}$ DIA) and lead (4 mm DIA) spheres were used as the porous layer, and oil and water as the saturating fluids. Thus the solid-to-fluid conductivity ratio, $\lambda$, varied between 0.25 and 60 . The experiments were performed in a rectangular test section and the Rayleigh number, $R a_{\mathrm{m}}$, was varied from 0 to 2000. Experimental results showed that the $N u_{\mathrm{m}}$-versus- $R a_{\mathrm{m}}$ relation depends on the thermal characteristics of the medium. Instead of a single curve, the 
authors found that the data could represented by a family of curves for different $\lambda$ values. The authors suggested that this is probably due to the non-validity of the assumption of an infinite heat transfer coefficient between the solid and the fluid phases. They also found that the convection heat transfer has two distinct regimes: a steady regime, and a fluctuating convective state that appears at Rayleigh numbers in the range 240-280.

Kaneko et al. [35] measured the heat transfer characteristics of saturated horizontal and inclined porous layers. They used two different kinds of silica sands as the porous material, and heptane and ethanol as the saturating fluids. For horizontal porous layers, they found the critical Rayleigh number for the heptane-sand system to be 40 which is very close to the theoretical value of $4 \pi^{2}$. However, the critical Rayleigh number for the ethanolsand system was found to be 28 , which is much lower than the theoretical value. They also found the $N u_{\mathrm{m}}$ and $R a_{\mathrm{m}}$ data for the heptane-sand system could be well correlated with the linear relation $N u_{\mathrm{m}}=R a_{\mathrm{m}} / 40 \pm 10 \%$ through the range of $R a_{\mathrm{m}}$ (up to 200) used by them. Their data for the heptane-sand system is in close agreement with Schenider's data for steel-oil and glass-water systems, as well as Combarnous' data for a sand-oil system.

Yen [36] conducted experiments to study the influence of the density inversion of the saturating fluid on the onset of convection. He used 3 and $6 \mathrm{~mm}$ DIA glass beads for the porous layer and distilled water as the saturating fluid. In order to study the effect of density inversion of water, Yen performed two different sets of experiments. In one set of experiments, the temperature of the top surface was maintained at either $4{ }^{\circ} \mathrm{C}$ or $8{ }^{\circ} \mathrm{C}$ in order to eliminate the density inversion of water at $4{ }^{\circ} \mathrm{C}$. In another set of experiments, the upper boundary was maintained at $0{ }^{\circ} \mathrm{C}$ in order to capture the effect of density inversion. For the first case, Yen found that the data could be represented by the relation $N u_{\mathrm{m}}=0.05 R a_{\mathrm{m}}^{0.82}$ while for the second case, the expression $N u_{\mathrm{m}}=0.024 R a_{\mathrm{m}}^{0.85}$ was found to be satisfactory. Yen also noted excellent agreement with the previous results of Schneider [28], Masuoka [31] and Combarnous [32]. Additionally, he found that the effect of density inversion on overall heat transfer is significant and that this effect decreases with increase in temperature.

Buretta [37], and Buretta and Berman [38] measured convective heat transfer coefficients for a saturated permeable layer that was either heated uniformly from below or by internally distributed heat sources. They used a cylindrical test section with 3,6 and $14.3 \mathrm{~mm}$ DIA glass spheres as the porous layer and de-mineralized water as the saturating fluid. Experiments were performed with several different layer heights and porosities with Rayleigh numbers up to $10^{4}$. For the case of uniform bottom heating, they found the critical Rayleigh number to be 38 which is close to the theoretical estimate of $4 \pi^{2}$. They also found that that the slope of their Nusselt-versus-Rayleigh number relationship at the critical Rayleigh number is remarkably close to the theoretical slope of $1 / 2 \pi^{2}$ predicted by 
Busse and Joseph [39]. Their experimental results were also found to be in good agreement with the upper bound estimates of Gupta and Joseph [40]. Excluding the data for the 14.3 mm DIA beads for $R a_{\mathrm{m}}>400$, they found that their data can be well correlated by,

$$
\begin{array}{ll}
\log \left(N u_{\mathrm{m}} \pm 0.076\right)=1.154 \log R a_{\mathrm{m}}-1.823 & \left(40<R a_{\mathrm{m}}<1000\right) \\
\log \left(N u_{\mathrm{m}} \pm 0.35\right)=0.835 \log R a_{\mathrm{m}}-1.124 & \left(40<R a_{\mathrm{m}}<1000\right) .
\end{array}
$$

Seki et al. [41] performed experiments to measure convective heat transfer in saturated porous layers in the high Rayleigh number regime. They used a rectangular test chamber for their experiments with $3 \mathrm{~mm}, 5.2 \mathrm{~mm}$ and $16.4 \mathrm{~mm}$ DIA glass beads as the porous medium, and distilled water, ethyl alcohol, fluorocarbon R-11 and transformer oil as the saturating fluids. Experiments were performed with a large number of porous layer heights ranging from $16.4 \mathrm{~mm}$ to $103 \mathrm{~mm}$. The authors found that the effects of particle diameter, porous layer depth and fluid Prandtl number on the heat transfer at high Rayleigh numbers are exceptionally large. For low values of the non-dimensional bead diameter, $\gamma$, the $N u_{\mathrm{m}}$-versus- $R a_{\mathrm{m}}$ curve is linear. For larger values of $\gamma$, this linear relationship is valid only for small $R a_{\mathrm{m}}$; at larger values of $R a_{\mathrm{m}}$ significant deviations from the linear relationship occur. They noted that this non-linear relationship for values of $P r_{\mathrm{f}}$ ranging from 1.1 to 7.3 can be expressed as,

$$
\begin{array}{cc}
N u_{\mathrm{m}}=0.1 P r_{\mathrm{m}}^{0.132} \gamma^{-0.655} R a_{\mathrm{m}}^{0.5} & \left(200<R a_{\mathrm{m}}<1400\right), \\
N u_{\mathrm{m}}=0.88 P r_{\mathrm{m}}^{0.132} \gamma^{-0.655} R a_{\mathrm{m}}^{0.2} & \left(1400<R a_{\mathrm{m}}<40000\right),
\end{array}
$$

From the above studies, it can be deduced that, in general, it is not possible to correlate experimental data by a single Nusselt-versus-Rayleigh number correlation. Experimental data for glass-water, glass-oil and heptane-sand systems are in good agreement with theoretical predictions but data for steel-oil, lead-water and ethanol-sand systems are not, especially at high Rayleigh numbers. This shows that the Nusselt-versus-Rayleigh number relation depends on the system being considered.

To explain the spread in experimental data, several hypotheses have been proposed. While Schneider [28] and Elder [29] proposed to use the fluid Nusselt and Rayleigh numbers for correlating experimental data at high Rayleigh numbers, Combarnous and Bories $[4,42]$ hypothesized that the observed discrepancy is due to the non-validity of the assumption of thermal equilibrium between the solid and fluid phases. To substantiate their hypothesis, Combarnous and Bories [42] developed a two equation model to account for heat transfer between the solid and fluid phases and used it in conjunction with the Darcy flow model. 
Using data from their experiments, they solved the resulting system of equations and found that at high Rayleigh numbers, the Nusselt number is a strong function of the solid-tofluid conductivity ratio, $\lambda$. Consequently, when $\lambda \gg 1$, heat transfer occurs mainly by conduction through the solid phase, and the condition of local thermal equilibrium is not satisfied leading to a scatter in the experimental data.

Prasad [43] and Prasad et al. [44] on the other hand proposed that the scatter in the experimental data occurs because the effective thermal conductivity is not properly represented. They noted that a plot of $N u_{\mathrm{m}} / P r$ versus $G r_{\mathrm{m}}$ does not depend on the thermal conductivity of the medium and that when plotted in these coordinates, the water-glass and the heptane-glass data coincide. This implies that the two systems have the same effective Prandtl number, despite the fact that $\lambda$ is very different for the two systems. They therefore concluded that the effective thermal conductivity of the medium is influenced by the intensity of the convection. To account for the enhancement of conductivity by convection, they proposed an ad hoc model where the effective conductivity, $k_{\text {eff }}$, is represented by a weighted average of the fluid conductivity and the stagnant effective thermal conductivity and is given by,

$$
k_{\text {eff }}=\varpi k_{\mathrm{f}}+(1-\varpi) k_{\mathrm{m}},
$$

where the weighting factor $\varpi$ is given as $\varpi=\left(1-1 / N u_{\mathrm{m}}\right)$. They noted that a lot of the scatter observed in the literature could be eliminated by using this form of the effective conductivity.

Another explanation was put forward by Somerton [45], who proposed that the data spread could be substantially reduced by taking into account the effect of the fluid inertia (quadratic drag) which becomes increasingly important with increase in the Rayleigh number. Somerton noted that by analogy with the Rayleigh-Bénard problem for single phase natural convection, a Prandtl number dependence is expected but is not accounted for in the Darcy flow model. Because the Prandtl number dependence in the Rayleigh-Bénard problem arises from fluid inertia effects, he proposed that the extended Darcy momentum equation is more appropriate to describe the flow. To demonstrate this dependence, he showed that non-dimensional form of the of the extended Darcy momentum equation yields two new non-dimensional numbers, the porous medium Prandtl number, $P r_{\mathrm{m}}$, and the Cozeny-Karmen number, $K C$, which are,

$$
\begin{gathered}
P r_{\mathrm{m}}=\frac{\nu}{\alpha_{\mathrm{m}}}, \\
K C=\frac{c_{K} H}{K},
\end{gathered}
$$


where $c_{K}$ is the Cozeny constant. By defining an effective porous medium Prandtl number, $P r_{\text {eff }}\left(P r_{\text {eff }}=K C \times P r_{\mathrm{m}}\right)$, Somerton showed that the data of Combarnous and Bories [4] could be collapsed on to three different curves for three different $P r_{\mathrm{m}}$ regimes.

To verify the hypothesis of Somerton [45], Catton and co-workers performed a series of experimental and numerical studies [46-48]. First, Jonsson and Catton [46] experimentally investigated natural convection in a saturated porous medium heated from below. Their experiments were conducted in a cylindrical test chamber with glass, stainless steel, and lead spheres for the porous medium and water, mercury, and silicone oil as the saturating fluids. With this set of solid and fluid phases, they were able to achieve a range of $4.17 \times 10^{-5} \leq P r_{\text {eff }} \leq 2$. They found that the Nusselt number is a very strong function of the effective Prandtl number when $P r_{\text {eff }}<0.1$; when $P r_{\text {eff }}>0.1$, however, the Nusselt number is independent of $P r_{\text {eff }}$. Based on this result, they proposed the following Nusselt-versusRayleigh number correlations,

$$
\begin{gathered}
N u_{\mathrm{m}}=0.118 R a_{\mathrm{m}}^{0.65} P r_{\mathrm{eff}}^{0.132} \quad\left(P r_{\mathrm{eff}} \leq 0.1\right), \\
N u_{\mathrm{m}}=0.147 R a_{\mathrm{m}}^{0.65} \quad\left(P r_{\mathrm{eff}}>0.1\right),
\end{gathered}
$$

Georgiadis and Catton [47], and Catton [48] numerically solved the equations for the extended Darcy flow model using a finite-difference Galerkin scheme. They found good agreement with the experimental results of Jonsson and Catton [46] when $R a_{\mathrm{m}}<200$ and $P r_{\text {eff }}=1.25$, which corresponds to the glass-silicone oil system. They also found good agreement when $R a_{\mathrm{m}}<200$ and $P r_{\text {eff }}=0.02$, which corresponds to the glass-water system. For the stainless steel-water system where $P r_{\text {eff }}=0.02$, however, their numerical solution did not converge for $R a_{\mathrm{m}}>200$. These studies show that while the effect of the Prandtl number is important, it is not the sole factor that causes a scatter in the $N u_{\mathrm{m}}$-versus- $R a_{\mathrm{m}}$ data.

Close [49], on the other hand, argued that the two principal factors affecting convective heat transfer in a saturated porous layer are: (i) heat transfer between the solid and fluid phases, and (ii) the effective stagnant conductivity of the saturated porous layer, $k_{\mathrm{m}}$. Therefore, these two factors must be considered carefully when attempting to correlate any experimental data. From the two-equation model proposed by Combarnous and Bories [42] to describe solid-to-fluid phase heat transfer, and a model for $k_{\mathrm{m}}$ proposed by Yagi and Kunii [50], Close derived five dimensionless parameters which govern convective heat transfer in a porous layer. Based on these, he proposed a parametric equation to fit the data of Schneider [28], Combarnous [33] and Buretta [37]. Using a least-squares method for fitting, he proposed the formula, 


$$
\begin{gathered}
\frac{N u_{\mathrm{m}}}{N u_{i}}=1.572 \times 10^{-2} \times R a^{0.344}\left(\frac{k_{\mathrm{f}}}{k_{\mathrm{so}}}\right)^{0.227}\left(\frac{H}{d_{p}}\right)^{0.446}\left(\frac{\phi}{1-\phi}\right)^{0.496} \operatorname{Pr}^{0.279}, \\
N u_{i}=1+\sum_{1}^{i} 2\left(1-\frac{4 i^{2} \pi^{2}}{R a_{\mathrm{m}}}\right) .
\end{gathered}
$$

Here $N u_{i}$ is the overall Nusselt number which is the sum of the Nusselt numbers due to independent contribution by each of the $i$ convective modes. Close noted that equation (2.14) is successful in correlating experimental data for $N u_{\mathrm{m}}<10$, but discrepancies are present for $N u_{\mathrm{m}}>10$. To explain them, he suggested that Darcy's law is inadequate in describing the pressure gradients within the bed. He also noted that the almost identical value of the exponents for $\operatorname{Pr}$ and $k_{\mathrm{f}} / k_{\mathrm{so}}$ in equation (2.14) implied that Somerton's claim that the solid-to-fluid heat transfer in the bed is negligible compared to fluid inertia was not necessarily correct.

Wang and Bejan [51] applied scale analysis to the mass, momentum and velocity conservation equations with the aim of developing a single relation to correlate experimental data reported in the literature. Their analysis led them to conclude that the Nusselt-versusRayleigh number relation is characterized by two distinct regimes: the Darcy regime and the Forchheimer regime. On the basis of their scale analysis, they found that in the Darcy flow limit, $N u_{\mathrm{m}} \sim(1 / 40) R a_{\mathrm{m}}$, while in the Forchheimer flow limit, $N u_{\mathrm{m}} \sim\left(R a_{\mathrm{m}} \times P r_{p}\right)^{1 / 2}$. Here $\operatorname{Pr}_{p}$ is a porous medium Prandtl number that represents the transition between the Darcy and Forchheimer regimes. To fit experimental data reported in the literature, Wang and Bejan proposed the correlation,

$$
N u_{\mathrm{m}}=\left[\left(\frac{R a_{\mathrm{m}}}{40}\right)^{-1.65}+\left\{1896.4\left(R a_{\mathrm{m}} P r_{\mathrm{p}}\right)^{1 / 2}\right\}^{-1.65}\right]^{-1 / 1.65}
$$

With the exception of data points in Schneider's results for $\operatorname{Pr}_{\mathrm{p}}=12$ and in Jonsson and Catton's data for $P r_{\mathrm{p}}=28$, the authors found good agreement with published experimental data.

Kladias and Prasad [52] conducted a numerical investigation into natural convection in a square cavity heated from below. They considered a Brinkman-Forchheimer extended Darcy model for the fluid motion and performed a parametric study to investigate the effects of the Darcy number, fluid Prandtl number, and the thermal conductivity ratio on the Nusselt number. From the numerical solution they found that for $\operatorname{Pr}>10$, the Prandtl number does not have any significant influence on fluid flow. For $\operatorname{Pr}<10$, on the other 
hand, the Nusselt number was found to increase with the fluid Prandtl number. They also found that an increase in the conductivity ratio, $\lambda$, substantially increases the Nusselt number and the critical Rayleigh number. In addition they found that the effect of the Prandtl number intensifies as $\lambda$ increases. Based on these observations they concluded that the conductivity ratio strongly affects the contribution of the inertia terms in the momentum equation.

Subsequently, Kladias and Prasad [53] conducted experiments to determine the accuracy of their numerical model. Their experiments were conducted in a cavity with an aspect ratio $A_{1}=5$ and a uniformly heated base. They used glass, acrylic and steel spheres as the porous material, and water, glycol and heptane as the saturating fluid. The selected solid-fluid combinations and particle sizes were aimed at maximizing the range of the thermal conductivity ratios, as well as the Darcy, Rayleigh, and Prandtl numbers. In general, they found that the experimental results confirmed the functional relationships predicted by their numerical solutions. They found excellent agreement for glass-water, glass-glycol, and acrylic-water systems at high Rayleigh and Darcy numbers. For the steel-water and steel-glycol systems, however, they found that the agreement is marginal at low Rayleigh numbers and rapidly diverges at high Rayleigh numbers. The divergence is largest for the steel-glycol system. This discrepancy was not improved even when a dispersive thermal conductivity proposed by Georgiadis and Catton [47] was incorporated in the numerical model.

The above discussion illustrates several important aspects of porous media natural convection. The most important aspect is the existence of a unique critical point for the onset of convection irrespective of the nature of the porous matrix, the saturating fluid or the problem domain. Beyond the critical point, however, all of these factors affect the overall heat transfer rates. The parameters that most significantly affect heat transfer in the high Rayleigh number $\left(R a_{\mathrm{m}}\right)$ range the solid-to-fluid conductivity ratio and the fluid Prandtl number. Although the exact effect of these parameters is unclear, their importance is readily apparent. In addition, these studies show that the effect of fluid inertia is significant at high Rayleigh numbers and cannot be ignored. These findings are extremely important for the present study as the aforementioned parameters are also likely to play a significant role for convection in a fluid-porous layer composite system.

\subsection{Natural convection in horizontal porous layers heated locally}

The problem of free convection in horizontal porous layers heated locally from below is now considered. The prototypical problem is one where only a portion of the bottom surface of the porous layer is heated and the remaining bounding surfaces are either adiabatic 


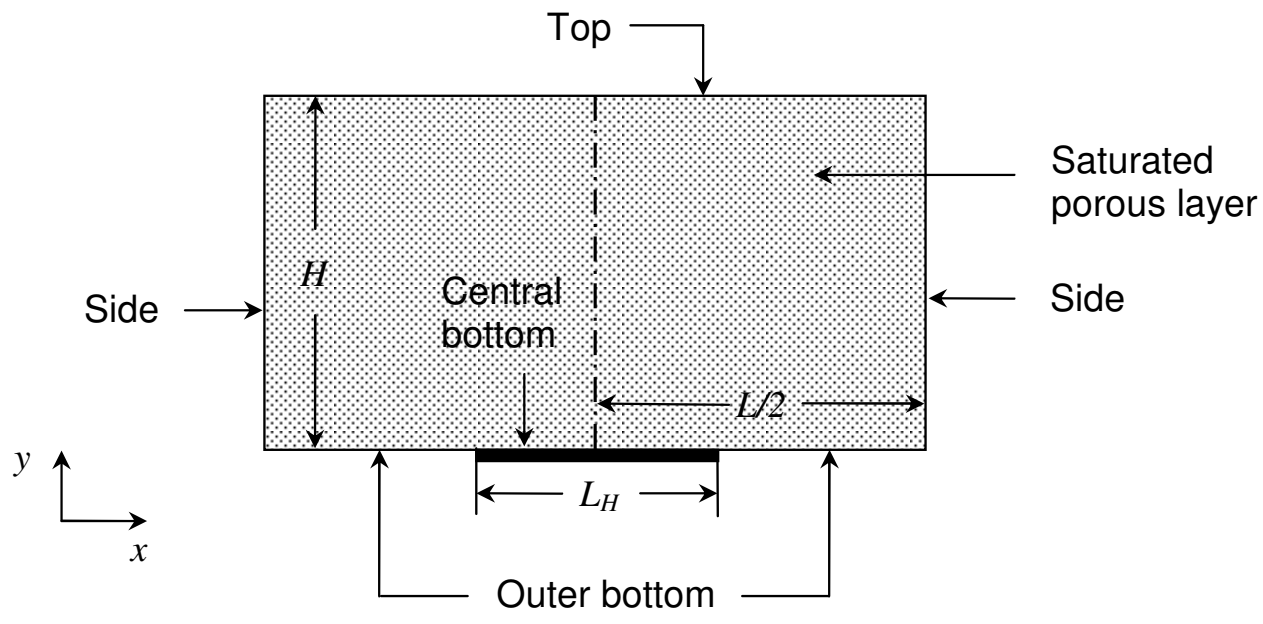

Figure 2.3: Two-dimensional domain of convection in a horizontal porous layer heated locally from below.

or isothermally cooled. Figure 2.3 shows a schematic diagram of the basic two dimensional problem where a rectangular cavity is filled with a saturated porous layer of height $H$ and width $L$ of which a central section of width $L_{H}$ is heated. Various thermal boundary conditions can be considered for this situation and these are listed in Table 2.1.

Elder [29] was the first to study natural convection in a horizontal porous layer with localized heating from below. He considered the case where all the walls of the cavity are held at a temperature $T_{C}$, except for the centrally heated portion which is held at a temperature $T_{H}$ (Case 1, Table 2.1). Using a finite-difference method, he numerically solved the governing equations for fluid flow and heat transfer and obtained the streamlines and isotherms for various values of the dimensionless heat source length, $\delta_{1}$. For a fixed aspect ratio $A=10$, when $\delta_{1}=1$, he found that the heated fluid rises as a thin column for $R a_{\mathrm{m}}$ as low as 50. For this heater size, recirculation of the fluid in the plume is very low (30\%). For $\delta_{1}=2$, two regions of opposite circulation are produced with a narrow fluid plume rising from the heated segment and a more diffuse, weaker return flow in the outer portion of the slab. For $\delta_{1}=3$, a pair of nearly square cells with opposite circulation, called Rayleigh cells, are produced over the heater and for $\delta_{1}=6$, three pairs of Rayleigh cells are produced. These results are shown in Figure 2.4. By performing a linear stability analysis, Elder found that Rayleigh cells would appear for $\delta_{1}=2,4$, and 6 .

Elder also examined the transient convection problem numerically and experimentally [54]. Using the same configuration as in his previous study, he studied the time dependent evolution of the flow and temperature fields when the localized region on the bottom is suddenly heated. He considered two different cases: (i) $A=4, \delta_{1}=2, R a_{\mathrm{m}}=400$, 
Table 2.1: The various thermal boundary conditions used for the local heating problem.

\begin{tabular}{ccccc}
\hline Case & Central Bottom & Outer Bottom & Sides & Top \\
\hline \hline 1 & $T=T_{H}$ & $T=T_{C}$ & $T=T_{C}$ & $T=T_{C}$ \\
2 & $T=T_{H}$ & $T=T_{C}$ & $\frac{\partial T}{\partial n}=0$ & $T=T_{C}$ \\
3 & $T=T_{H}$ & $\frac{\partial T}{\partial n}=0$ & $\frac{\partial T}{\partial n}=0$ & $T=T_{C}$ \\
4 & $\frac{\partial T}{\partial n}=-\frac{q^{\prime \prime}}{k}$ & $\frac{\partial T}{\partial n}=0$ & $\frac{\partial T}{\partial n}=0$ & $T=T_{C}$ \\
5 & $T=T_{H}$ & $T=T_{C}$ & $T=T_{C}+\frac{y}{H}\left(T_{T}-T_{C}\right)$ & $T=T_{T}$ \\
\hline
\end{tabular}

$0 \leq t^{*} \leq 0.1$, and (ii) $A=10, \delta_{1}=8, R a_{\mathrm{m}}=200,0 \leq t^{*} \leq 0.4$. In the first case he found that flow evolution begins with the formation of a pair of eddies with counter-circulations at the edge of the heater producing a rapidly rising column of hot fluid. With time another pair of eddies forms near the edges leading to a rapid re-adjustment of the flow field there. A small double eddy is then formed at the center of the heater which eventually grows and merges with the end eddy to form a single circulating cell in the half cavity. The bulk of the heat is carried by the hot plume rising from the center of the heater. In the second case, the initial onset of convection is identical to the first case with a pair of eddies forming at the edge of the heater. However, in this case, Elder found that successive circulating cells grow above the heater starting from the edge and moving towards the center with an approximately constant velocity. For his experiments, Elder used a Hele-Shaw cell, $5 \mathrm{~cm}$ $\times 20 \mathrm{~cm}$ with a plate spacing of $4.0 \mathrm{~mm}$, and silicone oil as the fluid. The heated section had a width of $10 \mathrm{~cm}$, while all the other surfaces were maintained at a constant temperature. The visualization streamlines were found to be qualitatively similar to the numerical simulations.

Horne and O'Sullivan [55] performed a numerical and experimental investigation of convection in a horizontal layer with localized heating from below in order to examine the stability of convective flow and determine the possibility of oscillatory or other unsteady flows. They considered the case where the sides of the cavity are adiabatic, and the top and outer bottom surfaces are maintained at a temperature $T_{C}$ (Case 2 in Table 2.1). They used a domain with an aspect ratio $A=2$ and $\delta=0.25,0.5$ and 0.75 . Their results show that for localized heating the system is self-restricting and either stabilizes into a steady multi-cellular flow or regularizes to a periodic oscillatory flow depending upon the values of $R a_{\mathrm{m}}$ and $\delta$. For $\delta=0.25$, they observed oscillatory flow at $R a_{\mathrm{m}}$ approximately greater than 450 while for $\delta=0.5$ this was seen at $R a_{\mathrm{m}}>480$. Within a single oscillation, 

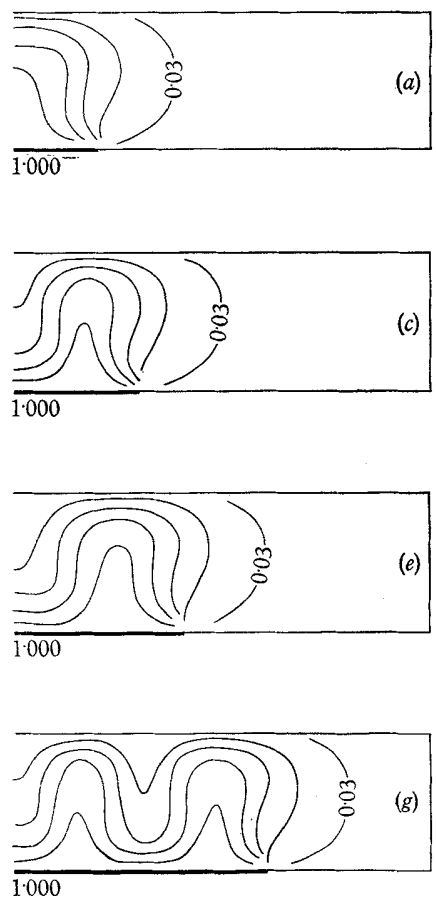
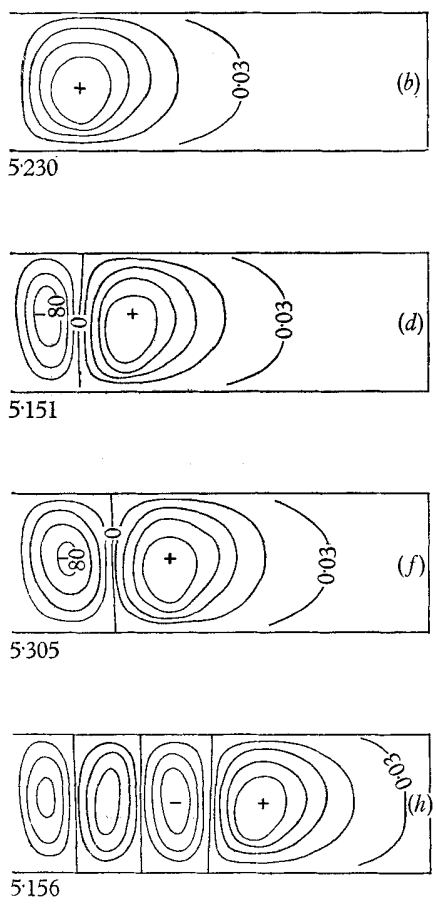

Figure 2.4: Isotherms (left column) and streamlines (right column) in a horizontal porous layer of aspect ratio $A=10$ and Rayleigh number $R a_{\mathrm{m}}=80$ for various values of the dimensionless heater length $\delta_{1}$. (a) and (b), $\delta_{1}=2 ;$ (c) and (d), $\delta_{1}=3$; (e) and (f), $\delta_{1}=4 ;(\mathrm{g})$ and $(\mathrm{h}), \delta_{1}=6$. (Elder [29]).

approximately mushroom shaped isotherms with plume like flow above the heated region were found to occur. This is shown in Figure 2.5. For $\delta=0.75$, however, the authors found no oscillatory flow in the Rayleigh number range $250<R a_{\mathrm{m}}<1250$. Instead, a tricellular steady flow pattern was seen. The authors suggest that this happens because in this case the length of the unheated segment is comparable to the cell width and thus reinforces the steady cellular flow pattern in spite of disturbances. Horne and O'Sullivan also performed experiments with a Hele-Shaw cell to verify their numerical findings. In their experiments they observed the same oscillatory flow pattern predicted by their simulations. The authors noted that this oscillatory flow is best exemplified by periodic observations of 'tongues' of fluid in the ascending and descending regions of the flow.

In a subsequent paper, Horne and O'Sullivan [56] extended their previous study to examine whether oscillatory flow in the porous layer is triggered by circulating thermal disturbances or by boundary layer instabilities. For this they considered the case where the top surface of the fluid is unconfined and compared the results with those obtained with a confined fluid. The setup and boundary conditions were identical to their earlier work except that they only studied the case where half the base was heated $(\delta=0.5)$. They found that the constant pressure boundary condition at the top surface for the case of 


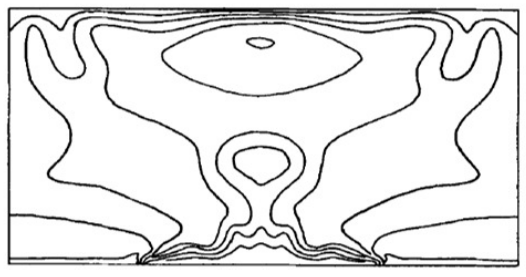

(a)

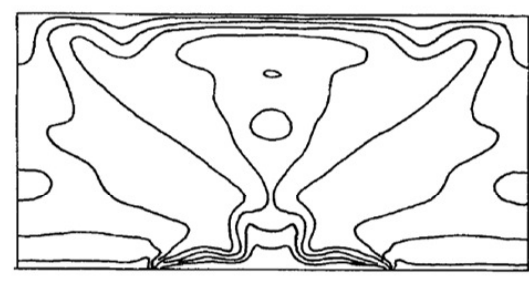

(c)

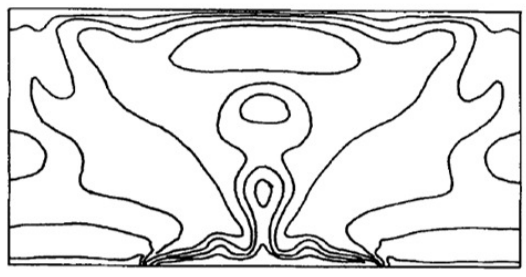

(b)

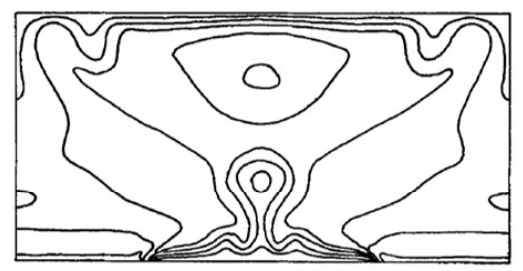

(d)

Figure 2.5: Computed isotherms during a single oscillation for a localized heating problem in a porous layer of aspect ratio $A=2, R a_{\mathrm{m}}=750$, heater length $\delta_{1}=0.5$ (Horne and O'Sullivan [55]).

unconfined fluid allows only cold descending fluid. As such there is no descending fluid, and hence no recirculation of thermal disturbances. In spite of this result, they found oscillatory flow patterns which are similar to those formed when the fluid is confined, although for the unconfined case, the ascending disturbances have larger amplitudes and velocities, and their onset occurs at lower values of $R a_{\mathrm{m}}$. Thus oscillatory disturbances have their origin in the thermal boundary layer on the heated bottom surface. It was also found that in both cases the frequency of the oscillation is proportional to $R a_{\mathrm{m}}^{3 / 2}$.

In a series of studies, Kulacki and co-workers examined the effect of a localized bottom heating on the flow patterns and heat transfer rates. Prasad and Kulacki [57] numerically studied the case when the central portion of the bottom surface is heated to a temperature $T_{H}$ while the remaining bottom surface area and the sides are adiabatic, and the top surface is kept at $T_{C}$ (Case 3, Table 2.1). To examine the effect of the size of the heat source and the horizontal extent of the porous layer, they considered two cases: (i) the width of the layer, $L$, was varied by keeping the height of the layer and length of the heater fixed $\left(\delta_{1}=1\right)$, and (ii) the aspect ratio is fixed $(A=2)$ for $\delta_{1}=0.4,1.0$ and 1.6. They found that for fixed $\delta_{1}$ and $A$, the conduction field is modified and a symmetrical circulatory flow field is setup for $R a_{\mathrm{m}}$ as low as 10 . With increasing the $R a_{\mathrm{m}}$ to 100 , the isotherms move upwards in a plume-like motion and produce a highly stratified region near the top surface. This plume-like flow is strengthened when $R a_{\mathrm{m}}$ is increased to 1000 . Due to strong buoyancy effects, they found that the velocities are higher near the heated segment and the returning cold fluid moves with a higher velocity towards the heated center. The 
temperature gradients and velocities were found to decrease continually with increase in horizontal distance from the heat source. The authors also found that heat transfer rate always increases with $R a_{\mathrm{m}}$. However, due to the thermal stratification, the rate of increase of $N u_{\mathrm{m}}$ (based on the area of the upper surface) decreased with increasing $R a_{\mathrm{m}}$. For fixed $A$, the overall heat transfer increases asymptotically with $\delta_{1}$. For a fixed $\delta_{1}$, however, an increase in the aspect ratio affects heat transfer in only a small region of the entire domain. They also found that for a fixed $R a_{\mathrm{m}}$ and $\delta_{1}, N u_{\mathrm{m}}$ increases with $A$ and then decreases to an asymptotic value. They did not, however, observe any oscillatory behavior.

In a subsequent study, Prasad and Kulacki [58] extended their earlier work to examine the effects of the size and strength of the heat source for a relatively large layer with $A=10$. Numerical results were obtained for $\delta=0.1,0.2,0.4,0.6$ and 0.8 and $R a_{\mathrm{m}}$ up to 1000. The authors found that the convective flow in the half-cavity is unicellular as long as the length of the heated segment is smaller than or equal to the height of the porous layer i.e. $\delta_{1}=2$. An increase in the size of the heat source beyond $\delta_{1}=2$ results in a multi-cellular flow behavior. In this case the main convective cell with clockwise flow moves to the right of the half cavity and a new counter-clockwise flow cell is produced on the left side, above the heater. Also, with increasing $R a_{\mathrm{m}}$ the authors found the isotherms to get more and more skewed. The local heat transfer rate at the upper surface was found to be a strong function of the number, size and strength of the convective cells. Also, the energy lost per unit area of the heat source was found to always increase with a reduction in the heater size for $\delta<0.4$. Similarly, the overall heat transfer coefficient was found to always increase with the size of the heat source and to be directly proportional to the length of the heater, $L_{H}$, when $\delta>0.4$ and $R a_{\mathrm{m}}>100$.

El-Khatib and Prasad [59] extended the above studies to consider the case where a linear thermal stratification is present in a horizontal porous layer that is locally heated from below. They considered a configuration where $T_{H}$ is the temperature of the centrally placed bottom heater, $T_{C}$ the temperature of the remaining bottom surface, $T_{T}$ the temperature of the top surface and the sides are subjected to a linear temperature gradient (Case 5, Table 2.1). The thermal stratification is thus expressed by the parameter $S_{t}=\left(T_{T}-T_{C}\right) /\left(T_{H}-\right.$ $\left.T_{C}\right)$. Numerical calculations were performed for $A=2, \delta=0.5,0 \leq S_{t} \leq 10$, and $R a_{\mathrm{m}}$ up to 1000 . They found that when $S_{t}=0$, i.e. when there is no stratification, the effect of an increase in $R a_{\mathrm{m}}$ is to produce a plume like flow above the heated region. With an increase in $R a_{\mathrm{m}}$, the domain affected by the heat source, as well as the amount of heat lost by the heater, increases. On the other hand, for a fixed value of $R a_{\mathrm{m}}$, an increase in $S_{t}$ reduces the convective velocities and hence the energy lost by the heat source. This indicates that there exists a critical value of $S_{t}$, as a function of $R a_{\mathrm{m}}$, beyond which the heat source gains energy. In fact under certain circumstances, it was seen that a part of the heated segment 
can gain energy while the other part is losing heat. Also, for $S_{t}>1$ the energy gained by the upper surface is almost independent of $R a_{\mathrm{m}}$.

Rajen and Kulacki [60], on the other hand, performed a numerical and experimental study of convection in a horizontal porous layer for the case where the central bottom heater emits a uniform heat flux instead of being maintained at a constant temperature. The remaining part of the lower surface and the side walls were adiabatic while the top surface was maintained at a constant temperature $T_{C}$ (Case 4, Table 2.1). They used finite element method to numerically solve the governing equations. For $A=16, \delta=1 / 12$ and $R a_{\mathrm{m}}=5$ they observed no convective flow in the right half cavity. However, for $R a_{\mathrm{m}}=50$, a plume like flow was seen above the heated surface although much of the domain was found to remain unaffected by the heat source. For $\delta=1 / 2$ and $R a_{\mathrm{m}}=50$ they found that several convective rolls were formed over the heater; the number and strength of these rolls was found to increase with $R a_{\mathrm{m}}$. For their experiments they used $6 \mathrm{~mm}$ DIA glass beads for the porous layer and distilled water as the saturating fluid. Experiments were performed in a rectangular test section and the strip heaters were used to vary the heated fraction of the base from $1 / 12$ to $1 / 2$. They obtained $N u_{\mathrm{m}}$-versus- $R a_{\mathrm{m}}$ relations for different values of $A$ and $\delta$ and found very good agreement between their theoretical predictions and experimental results. An interesting aspect observed by them was that the inflexion in the $N u_{\mathrm{m}}$ versus Ram curve observed for a fully heated bottom disappears when the base is partially heated. They also found that for a fixed $A$ and $R a_{\mathrm{m}}$, an increase in $\delta$ decreases the Nusselt number.

Lai and Kulacki conducted an experimental study of free and mixed convection with localized heating [61]. Experiments were performed using $3 \mathrm{~mm}$ DIA glass beads and water as the saturated porous medium. The porous layer under consideration had an aspect ratio of $A=21$ and a constant depth of $5.08 \mathrm{~cm}$. For natural convection, their measured heat transfer coefficients were found to be smaller than those predicted by numerical simulations for $\delta_{1}=1$, and larger for $\delta_{1}=3$ and 5. Overall, however, they found good agreement between their experimental results and theoretical predictions. For natural convection, they found that the Nusselt-versus-Rayleigh number relation is of the form $N u_{\mathrm{m}}=0.269 R a_{\mathrm{m}}^{0.451}$. The authors noted that improved agreement between their measurements and the predicted values could be obtained by using the effective conductivity, $k_{\text {eff }}$ (equation 2.9 ), for defining the Nusselt number. The authors also did not observe the oscillatory behavior predicted by Elder [29, 54] and Horne and O'Sullivan [55, 56].

Lai and Kulacki [62] also performed a numerical study of steady-state natural convection in two-dimensional layered porous media heated partially from below. In their study, the central bottom heater was kept at a constant temperature, the remaining bottom surface and the sides were adiabatic and the top surface was at $T_{C}$. The boundary conditions at 
the interface of the two porous layers were the continuity of temperature, pressure, normal flow and heat flow. A finite difference scheme was used to solve the governing equations. Calculations were made over a wide range of permeability ratios $0.01<K_{1} / K_{2}<100$ and layer height ratios, $H_{1} /\left(H_{1}+H_{2}\right)$ (subscripts 1 and 2 refer to the bottom and top layer, respectively). The authors found that for $H_{1} /\left(H_{1}+H_{2}\right)=0.25,0.5$ and 0.75 , the flow and temperature fields for a layered structure with $K_{1} / K_{2}>1$ are completely different from those with $K_{1} / K_{2}<1$. Heat transfer always begins as conduction in the less permeable layer and as convection in the layer with higher permeability. With an increase in the base Rayleigh number (Rayleigh number for the lower layer), $R a_{\mathrm{m} 1}$, the convection from the more permeable layer begins to penetrate the less permeable layer and eventually both the layers reach a convective mode for sufficiently high values of $R a_{\mathrm{m} 1}$. For $K_{1} / K_{2}<1$, this penetration is in the form of an inverse plume. For fixed $R a_{\mathrm{m} 1}$, the strength of the circulation in the convective cell was found to increase with the height ratio. For $K_{1} / K_{2}>1$, the overall Nusselt number was found to be less than that for a homogeneous porous layer but greater than that for $K_{1} / K_{2}<1$. Also for a given $R a_{\mathrm{m} 1}$, the Nusselt number was found to increase with the sub-layer thickness ratio for $K_{1} / K_{2}>1$. However, for $K_{1} / K_{2}<1$, the Nusselt number first decreases with the thickness ratio until the convection cells are formed in both layers, and then it increases with the thickness ratio.

Jendoubi and Kulacki [63] extended the above work to consider mixed convection in a doubly layered porous medium heated locally from below and above. The primary aim of their study was to ascertain whether the heat transfer results could alone be used to determine whether the heating is from the top or the bottom. Their problem set up was identical to that of Lai and Kulacki [62], and they used a finite volume method to numerically solve the governing equations. Results were obtained for a fixed aspect ratio $A=20$ and various values of the permeability ratio, $K_{1} / K_{2}$, conductivity ratio, $k_{1} / k_{2}$, height ratio, $H_{1} /\left(H_{1}+H_{2}\right)$ (subscripts 1 and 2 refer to the bottom and top layer, respectively), and non-dimensional heater length, $\delta_{1}$. For the special case of bottom heating with no external flow, i.e., natural convection in a two-layer composite medium, the authors found that Nusselt number depends strongly on both the Rayleigh number and the height ratio. For $K_{1} / K_{2}>1$, increasing the height ratio increases the Nusselt number especially at higher Rayleigh numbers. Similarly for a given height ratio and conductivity ratio, increasing $K_{1} / K_{2}$ leads to an increase in the Nusselt number. For $K_{1} / K_{2}=1$, i.e. for a single porous layer, the Nusselt number was found to decrease with increase in the heater length particularly at high Rayleigh numbers.

The studies of convection in porous layers heated locally from below show how presence of a localized heat source at the base affects the temperature and velocity profiles as well as the overall heat transfer rates. In particular, these studies show that there exists 


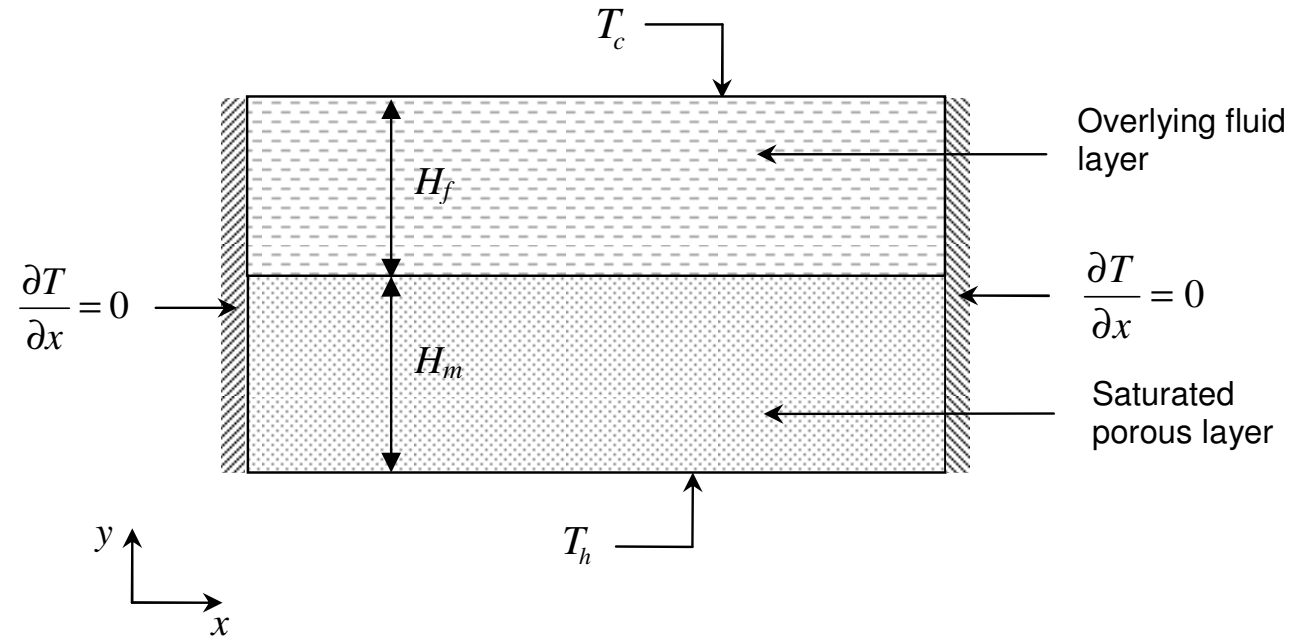

Figure 2.6: Schematic of the problem of natural convection in superposed fluid and porous layers heated uniformly from below.

a complex relationship between the heater length ratio and the aspect ratio such that for every aspect ratio there is a particular heater length for which the overall heat transfer rate is a maximum. These two parameters must thus be carefully considered when studying convection in a composite system with a localized heat source. In addition, the studies of convection in layered porous media are of immense relevance to the present study because the problem being investigated is a special case of the layered media problem for the limiting case of $K_{2} \rightarrow \infty$. As there are no available studies relevant to the present problem, these past studies can give significant insight into the nature of the results that can be expected from this study.

\subsection{Convection in fluid-superposed porous layers heated uniformly}

Having examined the literature for convection in a single porous layer heated from below, we now look at the literature on convection in fluid-superposed porous layers. For this problem, the literature almost exclusively points to the case where the bottom surface is uniformly heated. The prototypical problem can be represented as shown in Figure 2.6. A saturated porous layer with an overlying fluid layer is held between two impermeable surfaces. The lower surface is held at a higher temperature than the upper surface and side walls are kept adiabatic.

Sun [7] was probably the first investigator to examine the onset of convection in this composite system and conducted a comprehensive analytical and experimental study of convective stability. Using the perturbation method, he predicted the critical Rayleigh numbers for a variety of thermal and hydrodynamic conditions at the surface. He also 
performed experiments to determine the onset of convection and considered the case where there is internal heat generation in the porous layer. He studied the influence of various parameters such as the layer depth ratio, porosity, permeability, density, and diffusivity and found that the predicted critical Rayleigh number for various bed height ratios compared well with his experimental data for $0.826<\eta<1$. He also found that for a fixed Rayleigh number, the Nusselt number decreases with an increase in the height of the porous bed.

This problem was later studied analytically by Nield [8] who used a linear stability analysis to predict the onset of convection in a two-layer system that extended infinitely in the horizontal direction. He applied the Beavers-Joseph [61] condition at the fluid-porous layer interface and included the possibility of a Marangoni effect at the deformable upper surface. Nield, however, did not solve the resulting tenth-order eigenvalue problem because of its tedious nature. Instead he obtained the stability criterion for limiting values of various parameters such as the fluid viscosity, height ratio, and conductivity ratio.

On the other hand, Rhee et al. [62] experimentally measured the heat transfer rates in a composite system consisting of a porous layer made up of heat generating particles that was cooled by a layer of fluid from above. Their experiments were conducted in a cylindrical test section with $6.35 \mathrm{~mm}$ DIA steel balls as the porous layer and distilled water as the saturating fluid. The sides and the bottom of the test section were kept insulated while the top surface was maintained at a constant temperature. The steel balls were inductively heated by a 10 turn, $210 \mathrm{~mm}$ ID induction coil that was placed around the lower section of the test section. The height of the particulate bed was either $26 \mathrm{~mm}(\eta=$ $0.244)$ or $52 \mathrm{~mm}(\eta=0.122)$ while the height of the overlying liquid layer was parametrically varied from 0 to $209 \mathrm{~mm}$. Their results showed that the critical Rayleigh number for the porous layer, $R a_{\mathrm{m}}$, decreases rapidly as the fluid-to-porous layer depth ratio, $\eta_{1}$, approaches unity; above this value of $\eta_{1}, R a_{\mathrm{m}}$ reaches an asymptotic value of 12 . In the absence of the overlying liquid layer, however, the critical Rayleigh number is 46 , indicating that the presence of the liquid layer facilitates the onset of convection. The presence of the liquid layer was also seen to increase the rate of heat transfer through the bed. For a liquid layer about one fifth the height of the porous layer, the authors observed a threefold increase in the heat transfer rate. The authors noted that this was due to the inflow and outflow of fluid from the porous bed. They also noted that for $\eta_{1}>1$, the heat transfer data can be correlated by the relation $N u_{\mathrm{m}}=0.19 R a_{\mathrm{m}}^{0.69}$.

The problem of a fluid layer overlying a volumetrically heated porous bed was studied analytically by Somerton and Catton [63] who conducted a linear stability analysis to predict the onset of convection in this system. Instead of using Darcy's law, they used Brinkman's extension of Darcy's law to model fluid flow in the porous layer. This allowed 
them to avoid using the Beavers-Joseph boundary condition [46] at the interface and instead use more generalized boundary conditions describing the continuity of velocity and shear stress at the interface. The resulting equations were solved approximately by using the Galerkin method. They found the following: (i) the critical wave number is a function of the fluid-to-bed depth ratio, $\eta_{1}$, the conductivity ratio, $\lambda$, and the Darcy number, $D a$; (ii) the sole parameter controlling the onset of convection is a single Rayleigh number for the entire system; (iii) a larger $\lambda$ tends to produce a more stable fluid layer while a large $D a$ tends to produce a less stable fluid layer; (iv) the presence of the overlying fluid layer is destabilizing and can drive fluid motion in the porous bed. They, however, did not measure the heat transfer characteristics of the system.

Poulikakos et al. [20] studied the problem of flow instability in a horizontal composite layer beyond the critical Rayleigh number regime. They numerically solved the full equations for transient flow and used the Beavers-Joseph boundary condition at the porous layer-fluid layer interface. In their study the authors used a fixed Prandtl number, thermal diffusivity ratio and porous-to-total layer height ratio $(\eta=0.5)$. The parameters varied were the aspect ratio, $A(0.4-2)$, the fluid Rayleigh number, $R a\left(10^{2}-10^{6}\right)$ and the Darcy number, $D a\left(10^{-7}-10^{-4}\right)$. They obtained plots showing the streamlines and isotherms for various values of these parameters and found that with increasing Rayleigh number, the velocities of the two-dimensional cellular rolls increase but their numbers remains fixed. Also with increasing Rayleigh number, a reversal in flow behavior of the rolls occurs. However the authors did not observe any significant change in the Nusselt number with increase in the Darcy number but found that it decreases with increasing aspect ratio. They also found that the critical Rayleigh number is in the range of 500-600. Beyond this range, the Nusselt-versus-Rayleigh number correlation is given by $N u \approx 0.129 R a^{0.33}$.

Poulikakos [21] extended the above study by using a general flow model to describe flow through the porous media. This model incorporated the Brinkman and Forchheimer extensions to the Darcy model to account for the effects of macroscopic shear and flow inertia. Poulikakos used a single set of conservation equations for both the fluid and the porous layers. This was accomplished by using a binary parameter which assumes the value zero in the fluid region and unity in the porous region, thus eliminating terms such as the Darcy term in the fluid region. By this methodology, no explicit account is needed at the interface between the fluid and the porous layers. Numerical solutions were obtained by using a finite-volume technique for various values of parameters such as the aspect ratio, $A(1-5)$, the height ratio, $\eta(0.1-0.8)$, the fluid Rayleigh number, $R a\left(10^{3}-10^{5}\right)$, the Darcy number, $D a\left(10^{-5}-10^{-3}\right)$ and the Forchheimer coefficient, $F(0-1.0)$. Figure 2.7 shows the streamlines and isotherms for one of these cases. Poulikakos found that the velocity of convection motion increases with an increase in the Rayleigh number when all 


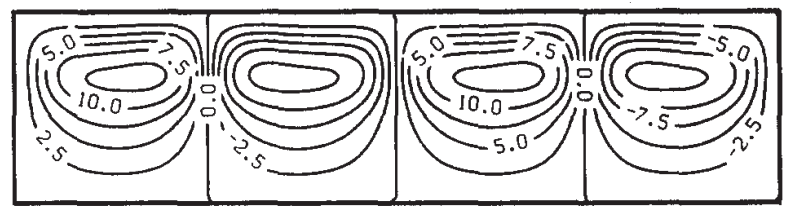

(a)

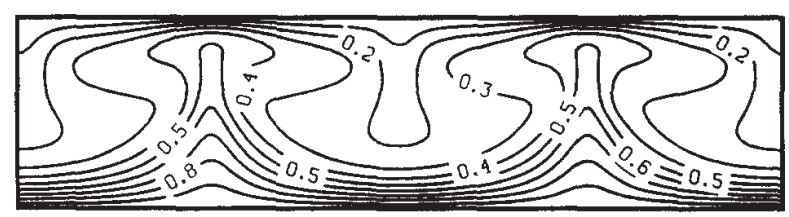

(b)

Figure 2.7: Streamlines (a) and isotherms (b) for natural convection in fluid superposed porous layer for $R a=10^{5}, A=4, \eta=0.5, D a=10^{-4}$, and $F=0.5$ (Poulikakos $[21])$.

other parameters are held constant. Also, the number of cells doubles when the Rayleigh number attains the value of $2 \times 10^{5}$. With increase in the height of the overlying fluid, a drastic increase in the overall Nusselt number occurs. Poulikakos noted that beyond a critical height ratio of $\eta \approx 0.75$ the flow field ceases to have any effect on heat transfer and heat removal is primarily by conduction. He also observed that an increase in $D a$ increases the Nusselt number.

In a series of studies, Chen and Chen $[9,10,22]$ investigated the convective instabilities and heat transfer characteristics of fluid-superposed porous layers both analytically and experimentally. In connection to the problem of directional solidification of concentrated alloys, Chen and Chen [9] first considered the problem of salt-finger convection in this two-layer system and used a linear stability analysis to determine the onset of finger convection. They used Darcy's law to model fluid flow in the porous layer and applied the Beavers-Joseph boundary condition at the fluid-porous layer interface. The additional effect of a salinity gradient was included in their conservation equations in order to model salt-finger convection. The authors used a fourth order Runge-Kutta shooting method to solve the resulting eigenvalue problem. To check the accuracy of their problem formulation and method of solution, they considered, as a special case, the pure thermal convection problem. They found that the parameters which affect the critical condition are the fluidto-porous layer height ratio, $\eta_{1}$, the Darcy number, $D a$, the thermal diffusivity ratio, $\epsilon$, and the Beavers-Joseph constant, $\hat{\alpha}$. For fixed values of $D a, \epsilon$ and $\hat{\alpha}$, they found that the marginal stability curve is bimodal. For $\eta_{1}<0.12$, the long wave branch is most unstable and the convection is dominated by the porous layer. For $\eta_{1}>0.12$, the short wave branch is the most unstable and the convection is dominated by the fluid layer. They also found 
that critical Rayleigh number decreases precipitously with increase in the depth ratio, $\eta_{1}$.

In a subsequent paper, Chen and Chen [10] conducted experiments to verify their earlier findings which were in disagreement with the experimental results of Sun [7]. For their experiments they used a rectangular test section with $3 \mathrm{~mm}$ DIA glass beads for the porous layer and water, $60 \%$ and $90 \%$ glycerin-water solutions, and $100 \%$ glycerin as the saturating fluids. The depth ratio $\eta_{1}$ was varied from 0.0 to 1.0. Fluids of increasing viscosity were used for cases with larger $\eta_{1}$ in order to keep the temperature difference across the test section within reasonable limits. The experimental results showed: (i) a precipitous decrease in the critical Rayleigh number as the depth of the fluid layer is increased from zero, and (ii) an eightfold decrease in the critical wavelength between $\eta_{1}=0.1$ and 0.2 . These findings confirmed their earlier theoretical predictions. They also visualized the convective cells and found them to be three dimensional.

Chen and Chen [22] further performed a computational analysis to study the heat transfer characteristics of the two-layer system analyzed in their previous studies. To model fluid flow in the porous layer, they considered the modified form of Darcy's equation which included the Brinkman and Forchheimer terms to account for viscous and inertial effects. The boundary conditions at the fluid-porous layer interface were the continuity of velocity, temperature, heat flux, normal stress and shear stress. The flow was assumed to be twodimensional and periodic in the horizontal direction with a wavelength equal to the critical value of the wavelength at onset, as predicted by the linear stability theory. The authors used a combined Galerkin and finite-difference method to numerically solve the governing equations. They found that convection remains steady for $R a_{\mathrm{m}} \leq 20 R a_{\mathrm{mc}}$ when the depth ratio, $\eta_{1}$, is varied between 0.1 and 1.0. For $\eta_{1}<0.13$ (the critical height ratio), the Nusselt number increases sharply with $R a_{\mathrm{m}}$, whereas at larger $\eta_{1}$ the increase is very moderate. Heat transfer rates predicted by the numerical scheme for $\eta_{1}=0.1$ and 0.2 show good agreement with their earlier experimental results [10].

Chen [11] conducted a linear stability analysis of the convective instability in the composite system with through-flow in the vertical direction. He found that in such a physical configuration, both stabilizing and destabilizing factors due to vertical throughflow could be enhanced allowing a more precise control of the buoyancy driven instability in either the fluid or the porous layer. For a depth ratio $\eta_{1}=0.1$, the onset of convection occurs in both fluid and porous layers, the relation between the critical Rayleigh number for the porous layer, $R a_{\mathrm{mc}}$, and the through-flow strength, $\zeta$, is linear, and the effect of the Prandtl number, $\operatorname{Pr}$, is insignificant. For $\eta_{1}>0.2$, the onset of convection was largely confined to the fluid layer, and the relation became $R a_{\mathrm{mc}} \sim \zeta^{2}$ for most of the cases considered except for $\operatorname{Pr}=1$ where the relation was $R a_{\mathrm{mc}} \sim \zeta^{3}$.

Further analytical studies were conducted by Chen and co-workers [12-14]. Chen 
et al. [12] studied the onset of thermal convection in a system consisting of a fluid layer overlying a porous layer with anisotropic permeability and thermal diffusivity. Flow in the porous medium was assumed to be governed by Darcy's law and the Beavers-Joseph condition was applied at the interface between the two layers. The linear perturbation equations were solved numerically by using a shooting technique based on a hybrid AdamsBashforth finite-difference method. The authors found that the effects of anisotropy on the onset of thermal convection are most profound for small values of the depth ratio, $\eta_{1}$. For fixed values of the vertical permeability in the porous medium, decreasing the value of the horizontal-to-vertical permeability ratio, $\xi$, led to stabilization of the superposed layer configuration because of increased resistance to motion in the porous medium. For larger values of $\eta_{1}$, the onset of motion was increasingly confined to the fluid layer, with the transport of heat through the porous layer primarily by conduction. Accordingly, the authors concluded that the influence of $\xi$ on the stability characteristics for larger $\eta_{1}$ was less significant than the effects of an anisotropic thermal conductivity.

Chen and Hsu [13] extended this work to cover a wide range of depth ratios, $\eta$, horizontal-to-vertical permeability ratios, $\xi$, and horizontal-to-vertical thermal diffusivity ratios, $\chi$. The authors found that for $\eta_{1}<0.1$, the critical Rayleigh number, $R a_{\mathrm{mc}}$, is essentially an explicit function of $\chi / \xi$ and the corresponding critical wave number, $a_{\mathrm{mc}}$, is a function of $(\xi \times \chi)^{1 / 4}$. Because for $\eta_{1}<0.1$ the porous layer dominates the two-layer system by convection, the anisotropic and inhomogeneous effects are significant as demonstrated by the well defined dependence of the critical Rayleigh number on the permeability and diffusivity ratios. For $\eta_{1}>0.1$, however, the authors did not obtain any explicit function for $R a_{\mathrm{mc}}$ or $a_{\mathrm{mc}}$ in terms of $\xi$ and $\chi$. They noted that because the onset of convection is largely confined to the fluid layer for $\eta_{1}>0.1$, the anisotropic and inhomogeneous effects characteristics of the porous layer are not significant.

On the other hand, Chen and $\mathrm{Wu}[14]$ numerically studied the effects of variable viscosity on the stability characteristics of the motionless overlying fluid. They used Darcy's law to model fluid flow in the porous layer and applied the Beavers-Joseph boundary condition at the interface. The viscosity variation was represented by the relation $\Xi=\log \left(\nu_{\max } / \nu_{\min }\right)$, where $\nu$ is the kinematic viscosity of the fluid. They found that the stability characteristics in terms of the critical Rayleigh number, $R a_{\mathrm{mc}}$, the critical wave number, $a_{\mathrm{mc}}$, and the critical flow patterns, are all profoundly influenced by the viscosity variation. The intrinsic features of the critical flow are mainly determined by the values of $\Xi$ and the depth ratio, $\eta_{1}$. The authors also identified three critical flow patterns on the basis of varying $\Xi$ and $\eta_{1}$ and found that the transition between any two of these three flow patterns is a bimodal instability.

Prasad and co-workers [24-26] conducted a series of experimental studies to visualize 
the flow patterns and measure the heat transfer characteristics of a fluid superposed porous medium uniformly heated from below. They used an immersion method to measure the refractive index of acrylic spheres, and matched it with the refractive index of the silicon fluid. This technique allowed the visualization of interesting flow interactions between the porous bed and overlying fluid layer. Aluminum particles were used for visualizing the flow patterns. In one set of experiments, Prasad and Tian [24] used glass beads of sizes 6, 15 and $24 \mathrm{~mm}$ DIA and varied the height ratio in the range $0.067 \leq \eta \leq 1$. The extended visualization studies showed that the flow field is highly complex and three dimensional, and active flow interactions between the overlying fluid and the packed beds were seen even when $R a_{\mathrm{m}} \sim \mathcal{O}(1)$. Strong, jet-like, vertical convective flows were seen to move from the underlying porous layer to the upper fluid layer. This results in a sharp drop in the critical Rayleigh number for the porous layer from $4 \pi^{2}$ as $\eta$ is reduced below unity. The authors reported that the observed flow patterns qualitatively matched those predicted by Poulikakos et al [20]. The authors also found that for small bead diameters, Nu decreases with increase in $\eta$. For larger bead diameters, $N u$ first decreases with $\eta$ until $\gamma_{\min }$ and then increases again. For the largest beads, a couple of inflexions on the $N u-\gamma$ curve were seen.

In another set of experiments [25], 12.7 and $25.4 \mathrm{~mm}$ DIA acrylic balls were used and the height ratio, $\eta$, was varied between 0 and 1 . The visualization studies showed that flow channels through large voids produce highly asymmetric and complicated flow structures. The number of convective rolls was found to depend on $\eta$. The heat transfer rate was found to generally increase with the Rayleigh number but its dependence on the non-dimensional particle size, $\gamma$, and the porous bed height was found to be very complex. This can be seen in Figure 2.8. Generally, the average Nusselt number increases with $\gamma$ (i.e., with increase in bead diameter), but it was found that there may exist some values of $\eta$ for which the heat transfer rate for a smaller $\gamma$ is higher. Also, the Nusselt number first decreases with an increase in $\gamma$ and reaches a minimum at $\gamma_{\min }$. Any further increase in porous layer height beyond this minimum value augments the heat transfer rate, and the Nusselt number curves show peaks at $\gamma_{\min } \leq \gamma \leq 1$. A general correlation for the Nusselt number was obtained in the form $N u=C R a^{n}$, where the constants $C$ and $n$ were found to depend on $\eta$ and $\gamma$.

Prasad [26] extended these studies further to include the influence of Prandtl number and different solid-fluid conductivity ratios. Experiments were conducted with acrylic, aluminum, and glass spheres for the porous bed, and two kinds of silicone oils and ethylene glycol as the saturating fluids. He found that the complex relationship between Nusselt number and porous layer height remains unaltered with a variation in thermal conductivity ratio and/or the Prandtl number. Also, an increase in thermal conductivity of the solid matrix was seen to enhance the heat transfer rate. Further, Prasad found that the composite 


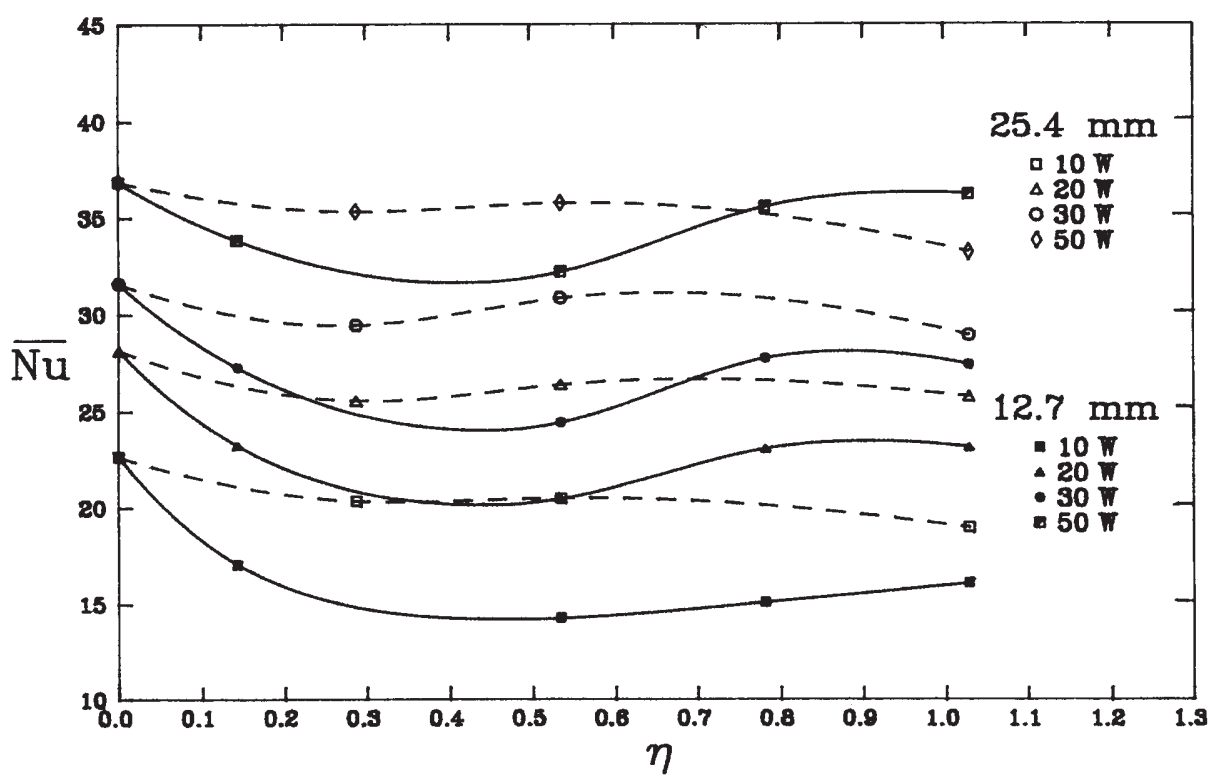

Figure 2.8: Overall Nusselt number versus height ratio for convection in a fluid-superposed porous layer. (Prasad et al. [25]).

fluid and porous layer can transport more energy than the just a pure fluid layer provided the porous matrix is highly permeable and the solid-to-fluid conductivity ratio is large. The effect of Prandtl number seemed to follow the trend reported for a cavity completely filled with the porous layer, particularly in high Darcy and Rayleigh number regimes.

Kim and Choi [23] numerically solved the stability problem for the onset of convection in an overlying fluid-porous layer composite system with the aim of validating the boundary conditions at the interface. Their scheme was able to accurately predict the number of circulating cells formed at the onset point. They found that when the depth ratio, $\eta_{1}$, is greater than 0.12 , the number of circulating cells increases continuously as the Rayleigh number increases, which in turn increases the Nusselt number continuously. However, for $\eta_{1}<0.1$, the recirculating cells constantly readjust their positions and sizes in the composite layer as the Rayleigh number increases. They also confirmed the abrupt and steep drop in the critical Rayleigh number with increase in the fluid depth ratio as observed by Chen and Chen [22]. However, they found that the number of cells in the supercritical convection regime does not increase monotonically with the Rayleigh number. Also, the corresponding Nusselt number variation is quite different from the numerical results of Chen and Chen $[22]$.

Recently, there has been some renewed interest in this topic. Carr and Straughan [67] provided an accurate numerical calculation for the instability that arises due to penetrative convection when water overlies and saturates a layer of porous material. In their study, the lower (porous) surface was maintained at $0{ }^{\circ} \mathrm{C}$ and the upper (fluid) surface 
was stress free with its temperature being above $0{ }^{\circ} \mathrm{C}$. This physical picture is capable of encompassing water at the density maximum of $4{ }^{\circ} \mathrm{C}$ in the layer and was thus capable of describing a model for patterned ground formation under water layers. To account for the fact that the density may have a maximum in the layer they adopt an equation of state which expresses the density in the buoyancy force as a quadratic function of temperature. They used a D2-Chebyshev tau method applicable to the porous-fluid layer thermal convection problem. Their results show significant convective penetration from the underlying porous layer into the upper fluid layer.

Steven [68] performed an experimental study to measure the heat transfer characteristics of a fluid superposed porous layer system heated from below. His experiments were performed in a cylindrical test chamber with $6 \mathrm{~mm}$ DIA glass beads as the porous layer and water as the saturating fluid. Heat transfer rates were measured for four different values of the height ratio $\eta$. Steven observed temperature fluctuations within the system at various radial and vertical locations. He also found that for a given Rayleigh number, the overall Nusselt number for a composite system is higher than that for a pure porous layer indicating the overlying layer enhances the overall rate of heat transfer. However, the overall Nusselt number does not change significantly with $\eta$ for different Rayleigh numbers.

Hirata and co-workers have recently published a few studies examining the problem of convective stability in superposed fluid and porous layers [16-19]. The primary aim of these studies has to been to investigate how the modeling of fluid flow in the porous region, and the boundary conditions at the fluid-porous layer interface affect the prediction of the onset of convection. In the first of these studies, Hirata et al. [16,17] carried out a linear stability analysis with three different modeling approaches: (i) a one-domain approach, in which the porous layer is treated as a pseudo fluid and the entire composite system is modeled with a single set of conservation equations; (ii) a two-domain approach where the fluid flow in the porous layer is modeled by Darcy's law, and the Beavers-Joseph condition is applied at the interface; and (iii) a modified two-domain approach where fluid flow in the porous layer is modeled by Brinkman's extension to Darcy's law, and the interfacial boundary conditions are the continuity of velocity, temperature, heat flux, normal stress and shear stress. The resulting eigenvalue problem was solved with a generalized integral transform technique. The authors found that the marginal stability curves obtained with the two-domain and modified two-domain approaches are in close agreement with each other, but differ significantly with the curves obtained with the one-domain approach, indicating that the mathematical formulation of the problem has great influence on the stability results. Their results also show that the effect of the including the Brinkman's term in the momentum equation is minimal.

An extension of the above study was subsequently published by Hirata et al. [18] 
in which the authors performed a linear stability analysis using the modified two-domain approach described above but with a different set of interfacial conditions. Instead of considering the continuity of shear stress at the interface, the authors incorporated a stress-jump boundary condition proposed by Ochoa-Tapia and Whitaker $[69,70]$. The other interfacial conditions were the continuity of velocity, temperature, heat flux and normal stress. The authors found that the stress-jump co-efficient strongly influences the bimodal marginal stability curves. For small fluid-to-porous layer height ratios, increasing the stress jump coefficient causes the convection in the fluid layer to become unstable. The convection in the porous layer, however, remains unaffected by the magnitude the stress-jump coefficient. Since convection in the fluid layer occurs due to perturbation of large wave numbers, the authors concluded that the stress-jump condition induces a more unstable situation at large wave numbers.

In another paper, Hirata et al. [19] repeated their earlier study on stability analysis with the one-domain, two-domain and modified two-domain approaches. The goal of this study was to examine the cause for the differences in the marginal stability curves obtained with the one-domain and the two-domain approaches. The conservation equations and the boundary conditions for the three approaches were the same as that used in their earlier studies. However, in their problem formulation for the one domain approach, they incorporated the hypothesis proposed by Kataoka [71] that the average properties of the porous medium like the porosity, permeability and effective diffusivity are Heaviside step functions and hence their differentiation must be considered in the meaning of distributions. Using this approach they found that the marginal stability curves for the one domain and two domain approaches are almost identical and show almost the same bimodal behavior irrespective of the depth ratio. Based on their results the authors concluded that the one and two domain approaches are identical provided that the one domain approach is properly interpreted mathematically, i.e., in the meaning of distributions.

From these studies it is evident that the ratio of the fluid layer height to the porous layer height exerts a significant influence on the flow and temperature profiles in both the fluid and the porous layers. It also has a significant influence on overall heat transfer rates through the composite system. Numerical studies have shown that the boundary conditions at the fluid-porous layer interface have a great effect on convective stability in such a system. Visualization studies of the flow patterns have also shown that there is significant interaction among the overlying fluid and the underlying porous layer. These results are extremely relevant to the present study as interfacial effects are also likely to play a significant role in determining flow patterns, temperature distributions and heat transfer rates when only a fraction of the base of such a composite system is heated. Hence careful attention must be paid in choosing the governing equations and boundary conditions during 
problem formulation.

\subsection{Closure}

From the preceding discussion it is clear that there exists a significant literature pertaining to the problem of natural convection in horizontal porous and fluid-superposed porous layers heated from below. These studies have examined various fundamental aspects of the problem and have enabled a thorough understanding of the principal governing criterion. In particular, they have identified how parameters such as the solid-to-fluid conductivity ratio, the fluid Prandtl number and fluid-to-porous layer height ratio affect convection heat transfer in these systems. However, the majority of them consider the case where the heat source is uniformly distributed along the bottom. The few studies that do consider a localized bottom heat source deal almost exclusively with saturated porous layers. There are apparently no published studies on natural convection in fluid-superposed porous layers heated locally from below. This is a significant shortcoming for the literature on natural convection in porous media. As mentioned earlier, a localized bottom heat source is often a more realistic boundary condition than a uniformly heated boundary. It is thus both important and timely to examine the problem of convection in a fluid-superposed porous layer with a concentrated bottom heat source. Such a study would give a more complete

picture of natural convection in fluid-superposed porous layers and complement existing studies on convection in such composite systems with uniform bottom heating. 


\section{Chapter 3}

\section{Mathematical Formulation and Numerical Method}

In this chapter, the mathematical formulation for the problem being studied is presented, and the numerical solution technique is discussed in detail. The governing equations for natural convection in two-dimensional fluid-superposed porous layers are described first. Thereafter, the boundary conditions for the problem are elucidated. In particular, the boundary conditions at the interface of the fluid and porous layers are discussed in detail. The one-domain formulation, which describes convection in a composite fluid-porous layer system with a single set of equations, is then derived, and the governing equations are presented in dimensionless form using the vorticity-stream function formulation. Finite difference equations are then derived using the control-volume method. The quadratic upwind interpolation for convective kinetics (QUICK) scheme [80] for handling convective terms, is also discussed in detail. Thereafter, a brief discussion on the direct method used for solving the system of linear equations is given. Also, the post-processing of the numerical data is discussed. Finally, an analysis is presented for the stability, convergence and accuracy of the numerical technique.

\subsection{Mathematical Formulation}

\subsubsection{Governing equations}

The dual fluid-porous layer system being examined in the present study is shown schematically in Figure 1. A horizontal fluid layer of thickness $H_{\mathrm{f}}$ extends over a saturated porous layer of thickness $H_{\mathrm{m}}$. The two-layer system is confined in a two-dimensional enclosure of overall height $H$ and length $L$, and is bounded on all four sides by impermeable boundaries. The two vertical walls are assumed to be adiabatic, the upper horizontal wall is held at a constant temperature $T_{C}$, and the lower boundary has a centrally heated portion 


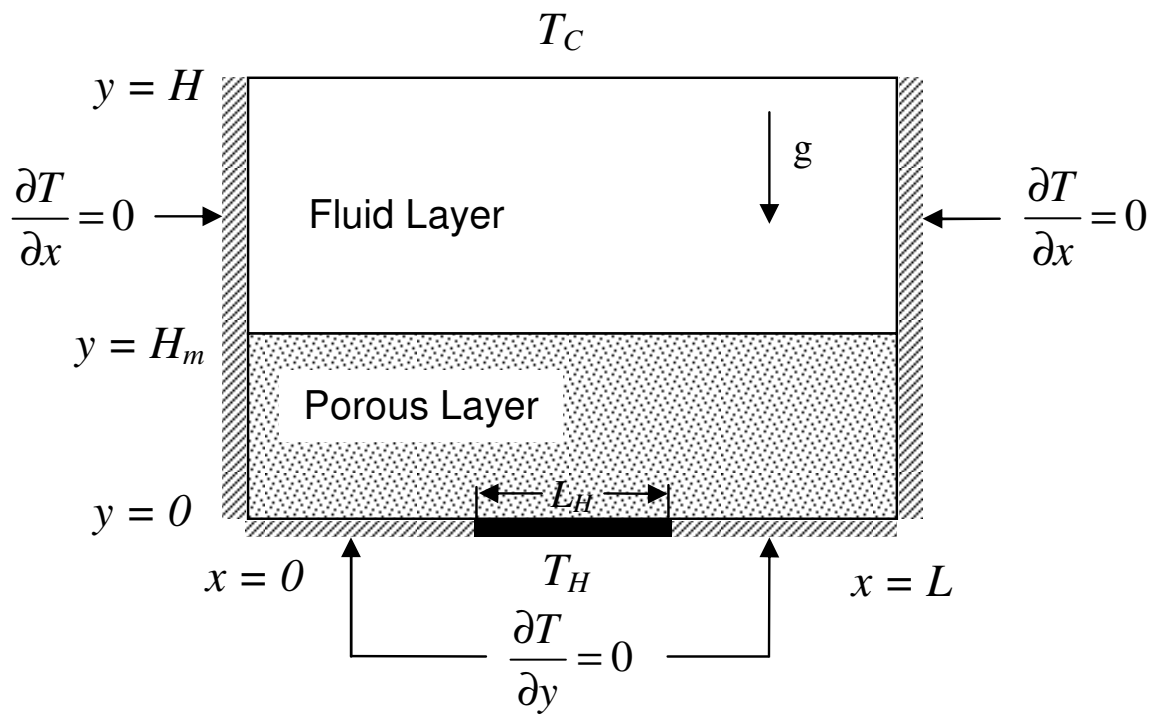

Figure 3.1: Domain of the composite two-layer problem.

of length $L_{H}$ that is held a constant temperature $T_{H}$ while the remaining portion is kept adiabatic. In addition, it is assumed that the system is potentially unstable, i.e., $T_{H}>T_{C}$ causing a buoyancy-driven flow instability. A Cartesian co-ordinate system is chosen with the $x$-axis horizontal and the $y$-axis pointing vertically upwards.

For the given system, two sets of continuity, momentum and energy equations describe natural convection heat transfer, one for each layer. For the fluid layer, the governing equations are,

$$
\begin{gathered}
\nabla \cdot \mathbf{u}_{\mathrm{f}}=0 \\
\rho\left[\frac{\partial \mathbf{u}_{\mathrm{f}}}{\partial t}+\left(\mathbf{u}_{\mathrm{f}} \cdot \nabla\right) \mathbf{u}_{\mathrm{f}}\right]=-\nabla P_{\mathrm{f}}+\mu_{\mathrm{f}} \nabla^{2} \mathbf{u}_{\mathrm{f}}+\beta \rho\left(T_{\mathrm{f}}-T_{0}\right) \mathbf{g} \\
\frac{\partial T_{\mathrm{f}}}{\partial t}+\left(\mathbf{u}_{\mathrm{f}} \cdot \nabla\right) T_{\mathrm{f}}=\alpha_{\mathrm{f}} \nabla^{2} T_{\mathrm{f}} .
\end{gathered}
$$

Here $\mathbf{u}_{\mathrm{f}}$ is the fluid velocity vector $\left(\mathbf{u}_{\mathrm{f}}=u_{\mathrm{f}} \mathbf{i}+v_{\mathrm{f}} \mathbf{j}\right), \rho$ denotes the fluid density, $T$ the time, $P_{\mathrm{f}}$ the fluid pressure, $\mu_{\mathrm{f}}$ the fluid dynamic viscosity, $\beta$ the coefficient of thermal expansion of the fluid, $T_{\mathrm{f}}$ the fluid temperature, $T_{0}$ a reference temperature and $\alpha_{\mathrm{f}}$ the fluid thermal diffusivity. The gravitational acceleration vector $\mathbf{g}$ vector points in the negative $y$ direction, and subscript 'f' denotes the fluid. In accordance with the Boussinesq approximation, the fluid density is assumed to be constant everywhere except in the buoyancy term of the 
momentum equation where its dependence on temperature is assumed to be linear and is,

$$
\rho=\rho_{0}\left[1-\beta\left(T_{\mathrm{f}}-T_{0}\right)\right]
$$

where $\rho_{0}$ denotes the fluid density at the reference temperature $T_{0}$.

The corresponding continuity, momentum and energy equations for the porous layer are,

$$
\begin{gathered}
\nabla \cdot \mathbf{u}_{\mathrm{m}}=0 \\
\rho\left[\frac{\partial \mathbf{u}_{\mathrm{m}}}{\partial t}+\left(\mathbf{u}_{\mathrm{m}} \cdot \nabla\right) \frac{\mathbf{u}_{\mathrm{m}}}{\phi}\right]=-\nabla P_{\mathrm{m}}+\mu_{\mathrm{eff}} \nabla^{2} \mathbf{u}_{\mathrm{m}}-\left(\frac{\mu \phi}{K}\right) \mathbf{u}_{\mathrm{m}} \\
-\left(\frac{\rho \phi F}{\sqrt{K}}\right)\left|\mathbf{u}_{\mathrm{m}}\right| \mathbf{u}_{\mathrm{m}}+\beta \rho\left(T-T_{0}\right) \mathbf{g}, \\
\sigma \frac{\partial T_{\mathrm{m}}}{\partial t}+\left(\mathbf{u}_{\mathrm{m}} \cdot \nabla\right) T_{\mathrm{m}}=\alpha_{\mathrm{eff}} \nabla^{2} T_{\mathrm{m}} .
\end{gathered}
$$

Here $\mathbf{u}_{\mathrm{m}}, T_{\mathrm{m}}$ and $P_{\mathrm{m}}$ represent the volume averaged velocity, temperature, and pressure in the porous medium, $\phi$ denotes the porosity, $K$ the permeability, $\mu_{\text {eff }}$ the effective viscosity of the Brinkman term and $F$ the Forchheimer inertial coefficient. The heat capacity ratio $\sigma$ is defined as,

$$
\sigma=\frac{\phi\left(\rho c_{p}\right)_{\mathrm{f}}+(1-\phi)\left(\rho c_{p}\right)_{\mathrm{so}}}{\left(\rho c_{p}\right)_{\mathrm{f}}}
$$

while the effective thermal diffusivity is defined as $\alpha_{\text {eff }}=k_{\text {eff }} /\left(\rho c_{p}\right)_{\mathrm{f}}$ where the effective conductivity $k_{\text {eff }}$ is,

$$
k_{\mathrm{eff}}=\phi k_{\mathrm{f}}+(1-\phi) k_{\mathrm{so}}
$$

The values of the parameters $K$ and $F$ depend on the nature of the porous medium. In this study, we consider the porous medium to comprise randomly packed spheres. For such a porous medium, the permeability and the Forchheimer coefficient are,

$$
\begin{gathered}
K=\frac{d^{2} \phi^{3}}{175(1-\phi)^{2}}, \\
F=\frac{1.75 \phi^{3 / 2}}{\sqrt{175}},
\end{gathered}
$$

where $d$ is the diameter of the spherical particles. 

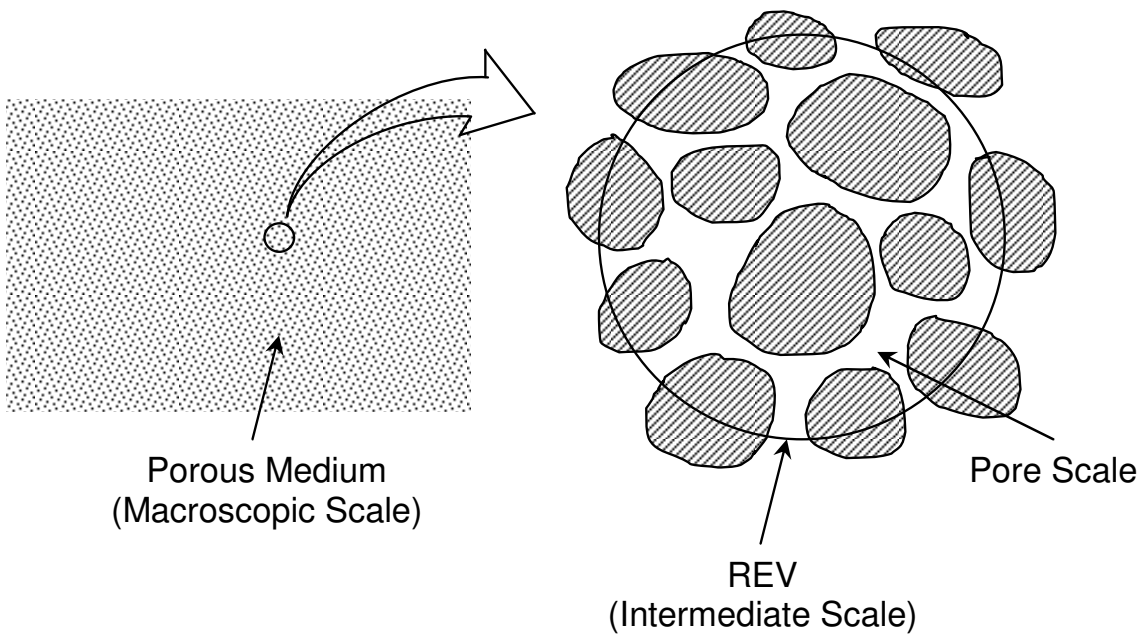

Figure 3.2: Schematic representation of a representative elementary volume (REV) showing the different scales of analysis in porous media.

It is instructive at this point to consider in some detail the form of the governing equations for transport through a porous medium as given above. The continuity, momentum and energy conservation equations for the porous layer are valid at the macroscopic scale of the porous medium and represent the volume averaged forms of these equations over a representative elementary volume (REV). This REV includes both the solid and the fluid components of the porous medium and is chosen in a manner such that it is much larger than the pore volume, but much smaller than the volume of the entire porous medium. A schematic representation of an REV is shown in Figure 3.2. These volume-averaged equations are useful from an engineering standpoint but are at the expense of the loss of detailed information concerning the microscopic structure of the porous medium. In particular, these equations do not account for the tortuosity of the porous medium, the nature of the boundaries between the solid and fluid phases, and the actual variation of quantities such as the pressure within the pores. However, the gross macroscopic effects of these factors are still retained in the form of parameters such as the porosity, permeability, and effective viscosity; these quantities can be related to the statistical properties of the porous medium under consideration as shown above. As such the volume averaged equations can adequately capture the effects of the microstructure of the porous medium at a macroscopic level without making the problem mathematically intractable. For this reason, the volume averaged governing equations are generally of immense importance in engineering analysis of porous media transport.

Another important aspect that needs to be discussed is the form of the energy equation given by equation (3.7). By writing a single energy equation for both the fluid and 
the solid phases, it has been implicitly assumed that a condition of local thermal equilibrium between the two phases exists in the porous layer. This is a reasonable assumption when the solid-to-fluid conductivity ratio, $\lambda$, is close to unity. For larger conductivity ratios, especially for $\lambda \gg 1$, however, this condition is not strictly true. In that case, separate energy equations are needed for the solid and the fluid phases and the thermal resistance between the phases needs to be taken into account. This two-equation model, however, makes the numerical solution much more involved. As such, it will not be considered in the present study. An equilibrium model such as the present one can, however, highlight several important aspects of the problem for large conductivity ratios. As a result, this model will be used to study problems with conductivity ratios as high as $\lambda=100$, though it must be borne in mind that the results are only for qualitative understanding and may not yield correct quantitative predictions.

\subsubsection{Boundary Conditions}

For the given problem, the appropriate boundary conditions at the system boundaries are,

$$
\begin{gathered}
u_{\mathrm{f}}=v_{\mathrm{f}}=0, \quad T_{\mathrm{f}}=T_{C}, \quad \text { at } \quad y=H, \\
u_{\mathrm{m}}=v_{\mathrm{m}}=0, \quad \begin{cases}T_{\mathrm{m}}=T_{H} & \text { at } y=0, \frac{L-L_{H}}{2} \leq x \leq \frac{L+L_{H}}{2}, \\
\frac{\partial T_{\mathrm{m}}}{\partial y}=0 & \text { at } y=0, \text { elsewhere, }\end{cases} \\
u=v=0, \quad \frac{\partial T}{\partial x}=0, \quad \text { at } \quad x=0, L .
\end{gathered}
$$

The initial boundary conditions can be written,

$$
u=0, \quad v=0, \quad T=0, \quad \text { at } \quad t=0 .
$$

In addition, the following boundary conditions need to be satisfied at the fluidporous layer interface,

$$
\begin{aligned}
\mathbf{u}_{\mathrm{f}} & =\mathbf{u}_{\mathrm{m}}, \\
-P_{\mathrm{f}}+2 \frac{\partial v_{\mathrm{f}}}{\partial y} & =-P_{\mathrm{m}}+\frac{2}{\phi} \frac{\partial v_{\mathrm{m}}}{\partial y} \\
\frac{\partial \mathbf{u}_{\mathrm{f}}}{\partial y} & =\frac{1}{\phi} \frac{\partial \mathbf{u}_{\mathrm{m}}}{\partial y}
\end{aligned}
$$




$$
\begin{gathered}
T_{\mathrm{f}}=T_{\mathrm{m}}, \\
k_{\mathrm{f}} \frac{\partial T_{\mathrm{f}}}{\partial y}=k_{\mathrm{m}} \frac{\partial T_{\mathrm{m}}}{\partial y} .
\end{gathered}
$$

The above boundary conditions express the continuity of velocity, normal and tangential shear stress, temperature, and heat flux across the fluid-porous layer interface. These boundary conditions are however the subject of much controversy and there is no consensus yet on which set of boundary conditions accurately captures the fluid dynamics and heat flow at the interface. Of particular interest are the boundary conditions which express the continuity of tangential velocity and tangential shear stress at the interface. As discussed in the previous chapter, several different approaches have been proposed to model the interfacial velocity and shear stress. Beavers and Joseph [64] demonstrated that the tangential velocity is discontinuous and that this discontinuity could be expressed in terms of a semi-empirical slip boundary condition. Ochoa-Tapia and Whitaker $[69,70]$ on the other hand have used volume averaging to model flow behavior at the interface and have proposed a stress-jump boundary condition to account for the discontinuity in the tangential shear stress at the interface. All these boundary conditions, however, have been derived for the special case of Poisseuille flow adjacent to a porous layer and can only be applied in conjunction with Darcy's law in the porous layer. The interfacial boundary conditions for buoyancy driven flow in composite fluid-porous systems, however, have not been clearly elucidated. In addition, boundary conditions that describe either the discontinuity of velocity or tangential shear stress at the interface involve an empirical parameter whose value must be determined experimentally.

Due to the lack of a consistent formulation for the interfacial boundary conditions, the continuity boundary conditions will be considered in the present study. A study by Singh and Thorpe [72] found that for buoyancy driven flow in composite layers, the particular form of the interfacial boundary conditions has little effect on the flow patterns and overall heat transfer rates for Darcy numbers up to $10^{-3}$. At higher Darcy numbers, Darcy's law breaks down, and boundary conditions modeling the discontinuity of velocity or shear stress can no longer be applied. However the continuity boundary conditions, which are used in conjunction with the Darcy-Brinkman formulation, can be used for all values of the Darcy number. These boundary conditions are therefore applicable for a wide range of porous media. The use of the continuity conditions has another advantage in that it allows one to use the one-domain formulation in which the two sets of governing equations for the fluid and porous layers can be combined into a single set of equations. In other words, both 
the porous and the fluid layers can be modeled as a single domain by one set of equations, the solution of which satisfies the continuity of velocity, stress, temperature, and heat flux at the interface. This approach significantly simplifies the numerical solution as no special modifications have to be made to the computer program to accommodate the interfacial conditions. Therefore the one-domain formulation will be used in the present study.

\subsubsection{One-domain Formulation}

The one-domain formulation requires a single set of governing equations for the entire composite domain. In the present study, these equations will be presented using the stream function-vorticity representation for the momentum equation. With this formulation, the pressure term in the momentum equation can be eliminated, which enormously simplifies the numerical solution. In order to derive the one-domain formulation, the following dimensionless parameters are defined,

$$
\begin{aligned}
& x^{*}=\frac{x}{H} \quad u^{*}=\frac{u}{\left(\alpha_{\mathrm{f}} / H\right)} \quad T^{*}=\frac{T-T_{C}}{T_{H}-T_{C}}, \\
& y^{*}=\frac{y}{H} \quad v^{*}=\frac{v}{\left(\alpha_{\mathrm{f}} / H\right)} \quad t^{*}=\frac{t}{\left(H^{2} / \alpha_{\mathrm{f}}\right)} \text {, }
\end{aligned}
$$

where the superscript ( )* denotes dimensionless quantities. Also, the dimensionless stream function and vorticity are,

$$
\begin{gathered}
\omega^{*}=\frac{\partial v^{*}}{\partial x^{*}}-\frac{\partial u^{*}}{\partial y^{*}} \\
u^{*}=-\frac{\partial \psi^{*}}{\partial y^{*}} \quad v^{*}=\frac{\partial \psi^{*}}{\partial x^{*}} .
\end{gathered}
$$

With the above definitions, the dimensionless stream function-vorticity equation is,

$$
\frac{\partial^{2} \psi^{*}}{\partial x^{* 2}}+\frac{\partial^{2} \psi^{*}}{\partial y^{* 2}}=\omega^{*}
$$

Note that the above equation satisfies the continuity equation identically.

Using the above definitions, the non-dimensional governing equations for the fluid layer are,

$$
\begin{gathered}
\frac{\partial \omega^{*}}{\partial t^{*}}+\left(\mathbf{u}^{*} \cdot \nabla\right) \omega^{*}=\operatorname{Pr} \nabla^{2} \omega^{*}+\operatorname{Ra} \operatorname{Pr} \frac{\partial T^{*}}{\partial x^{*}}, \\
\frac{\partial T^{*}}{\partial t^{*}}+\left(\mathbf{u}^{*} \cdot \nabla\right) T^{*}=\nabla^{2} T^{*}
\end{gathered}
$$


where the Rayleigh and the Prandtl numbers are,

$$
\begin{gathered}
R a=\frac{g \beta H^{3}\left(T_{H}-T_{C}\right)}{\nu \alpha_{\mathrm{f}}}, \\
\operatorname{Pr}=\frac{\nu}{\alpha_{\mathrm{f}}} .
\end{gathered}
$$

Similarly, the governing equations for the porous layer are,

$$
\begin{aligned}
& \phi \frac{\partial \omega^{*}}{\partial t^{*}}+\left(\mathbf{u}^{*} \cdot \nabla\right) \omega^{*}=\operatorname{Pr} \phi \nabla^{2} \omega^{*}-\left(\frac{\operatorname{Pr} \phi^{2}}{D a}+\frac{F \phi^{2}}{\sqrt{D a}}\left|\mathbf{u}^{*}\right|\right) \omega^{*} \\
&+\frac{F \phi^{2}}{\sqrt{D a}}\left(u^{*} \frac{\partial\left|\mathbf{u}^{*}\right|}{\partial y^{*}}-v^{*} \frac{\partial\left|\mathbf{u}^{*}\right|}{\partial x^{*}}\right)+\operatorname{RaPr} \phi^{2} \frac{\partial T^{*}}{\partial x^{*}}, \\
& \sigma \frac{\partial T^{*}}{\partial t^{*}}+\left(\mathbf{u}^{*} \cdot \nabla\right) T^{*}=\kappa \nabla^{2} T^{*},
\end{aligned}
$$

where the Darcy number and conductivity ratio are,

$$
\begin{gathered}
D a=\frac{K}{H^{2}}, \\
\kappa=\frac{k_{\mathrm{m}}}{k_{\mathrm{f}}} .
\end{gathered}
$$

To derive the one-domain formulation for the vorticity transport equation, one needs to consider the definitions of the permeability, $K$, and Darcy number, $D a$. In the fluid layer the porosity has no meaning, i.e. $\phi \equiv 1$, and the permeability and Darcy number are,

$$
\phi \rightarrow 1 \Rightarrow K \rightarrow \infty, D a \rightarrow \infty
$$

Substituting the above in equation (3.29), it can be seen that the vorticity transport equation for the porous layer reduces to that for the fluid layer in the limit of the porosity approaching unity. Thus equation (3.29) alone is sufficient to represent vorticity transport in the composite fluid-porous layer system. The porosity thus acts as a switching parameter that allows the vorticity transport equation to take on the appropriate form depending on whether the solution domain lies in either the fluid or the porous layer. This conditionality can be expressed as,

$$
\phi= \begin{cases}1, & \text { in the fluid layer } \\ 0, & \text { in the porous layer. }\end{cases}
$$


The one domain formulation for the energy equation can be derived in a similar manner. For the fluid layer the thermal conductivity ratio, $\sigma$, stagnant conductivity, $k_{\mathrm{m}}$, and the conductivity ratio, $\kappa$, can be written,

$$
\phi \rightarrow 1 \Rightarrow \sigma \rightarrow 1, k_{\mathrm{m}} \rightarrow k_{\mathrm{f}}, \kappa \rightarrow 1
$$

Thus in the limiting case of the porosity approaching unity, the energy equation for the porous layer reduces to the energy equation for the fluid layer. This equation is therefore the one-domain representation of the energy equation

Therefore, for the one-domain formulation, the dimensionless governing equations are,

$$
\begin{gathered}
\frac{\partial \omega}{\partial t}+(\mathbf{u} \cdot \nabla) \omega=\operatorname{Pr} \phi \nabla^{2} \omega+\operatorname{RaPr} \phi^{2} \frac{\partial T}{\partial x} \\
\phi \frac{\partial \omega}{\partial t}+(\mathbf{u} \cdot \nabla) \omega=\operatorname{Pr} \phi \nabla^{2} \omega-\left(\frac{\operatorname{Pr} \phi^{2}}{D a}+\frac{F \phi^{2}}{\sqrt{D a}|\mathbf{u}|) \omega}\right. \\
+\frac{F \phi^{2}}{\sqrt{D a}}\left(u \frac{\partial|\mathbf{u}|}{\partial y}-v \frac{\partial|\mathbf{u}|}{\partial x}\right)+\operatorname{RaPr} \phi^{2} \frac{\partial T}{\partial x}, \\
\sigma \frac{\partial T}{\partial t}+(\mathbf{u} \cdot \nabla) T=\kappa \nabla^{2} T
\end{gathered}
$$

where the superscript, ()$^{*}$, has been dropped for convenience. Dimensionless quantities will henceforth be written without any superscripts.

The corresponding dimensionless boundary conditions are,

$$
\begin{aligned}
& u=v=0, \quad \omega=\frac{\partial^{2} \psi}{\partial y^{2}}, \quad T=0, \quad \text { at } \quad y=1, \\
& u=v=0, \quad \omega=\frac{\partial^{2} \psi}{\partial y^{2}}, \quad \begin{cases}T=1 & \text { at } \quad y=0, \frac{L-L_{H}}{2 H} \leq x \leq \frac{L+L_{H}}{2 H} \\
\frac{\partial T}{\partial y}=0 & \text { at } y=0, \text { elsewhere }\end{cases} \\
& u=v=0, \quad \omega=\frac{\partial^{2} \psi}{\partial x^{2}}, \quad \frac{\partial T}{\partial x}=0, \quad \text { at } \quad x=0, A
\end{aligned}
$$

The dimensionless initial conditions are,

$$
u=v=0, \quad \psi=\omega=0, \quad T=0, \quad \text { at } \quad t \leq 0
$$




\subsection{Numerical solution of the governing equations}

An important question that arises during numerical simulations is the accuracy of the results in describing the actual physical process being studied. The numerical method selected must ensure that the discretized equations possess the physical properties of the original differential equations while giving stable, consistent, and accurate solutions. It is thus vital to select an appropriate numerical technique. In this section, the numerical solution technique used in the present study is discussed in detail. In addition, various aspects of the numerical solution, such as the effects of spatial discretization, convergence criterion, and truncation and round off errors, will be discussed. Because all of these factors combine to limit the accuracy of the simulations, a thorough understanding of these issues will enable correct interpretation of the results.

\subsubsection{Derivation of the finite difference equations}

The first step in the numerical solution of the governing equations is to derive the discretized form of the governing differential equations. Usually this can be done using several different techniques. In the present study, the control-volume formulation developed by Spalding and co-workers $[73,74]$ is used. In this method, the computational domain is covered with a set of non-overlapping control-volumes, each of which contains a single grid point. The governing equations are then integrated over each control-volume and piecewise profiles expressing the variation of the dependent variable between the grid points are used to evaluate the resulting integrals. The result is a set of discretized equations containing the values of the dependent variable for a set of grid points. This approach ensures that the conservation laws are satisfied over any control-volume and, thus, over the entire calculation domain. This characteristic exists for any number of grid points, not just in a limiting sense that the number of grid points has to be sufficiently large. Thus, even coarse-grid solutions exhibit integral balance which makes this technique highly attractive and useful.

The equations to be solved for the present problem can be classified into two major classes of differential equations. The stream function-vorticity equation is an elliptic partial differential equation similar to Poisson's equation while the vorticity transport and the energy equations are advection-diffusion equations, which have mixed characteristics. These two classes of differential equations have different solution techniques and each will be considered separately.

\subsubsection{Stream function-vorticity equation}

The solution of the stream function-vorticity equation will be considered first. Rewriting equation (3.36), 


$$
\frac{\partial}{\partial x}\left(\frac{\partial \psi}{\partial x}\right)+\frac{\partial}{\partial y}\left(\frac{\partial \psi}{\partial y}\right)-\omega=0
$$

In order to derive the finite-difference equations, consider a single control-volume as shown in Figure 3.3. The focus is on the grid point $P$ which has grid points $E, W, N$, and $S$ as its neighbors. Here $E$ denotes the east side, i.e., the positive $x$-direction and $W$ denotes the negative $x$-direction. Similarly, $N$ and $S$ denote the positive and negative $y$-directions, respectively. The control volume faces are denoted by $e, w, n$, and $s$, and the control volume corners are denoted by $n e, s e, s w$, and $n w$. In the present study a uniform grid is considered. As such, the control volume faces are located exactly midway between the grid points. Integrating equation (3.43) over a control volume one has,

$$
\begin{gathered}
\int_{s}^{n} \int_{w}^{e} \frac{\partial}{\partial x}\left(\frac{\partial \psi}{\partial x}\right) \mathrm{d} x \mathrm{~d} y+\int_{w}^{e} \int_{s}^{n} \frac{\partial}{\partial y}\left(\frac{\partial \psi}{\partial y}\right) \mathrm{d} x \mathrm{~d} y-\int_{s}^{n} \int_{w}^{e} \omega \mathrm{d} x \mathrm{~d} y=0, \\
\text { or, } \quad\left[\left(\frac{\partial \psi}{\partial x}\right)_{e}-\left(\frac{\partial \psi}{\partial x}\right)_{w}\right] \Delta y+\left[\left(\frac{\partial \psi}{\partial y}\right)_{n}-\left(\frac{\partial \psi}{\partial y}\right)_{s}\right] \Delta x-\bar{\omega} \Delta x \Delta y=0,
\end{gathered}
$$

where $\bar{\omega}$ represents the average value of $\omega$ over the control volume. In order to evaluate the derivatives in equation (3.45) suitable profile assumptions for the stream function are needed. Assuming a piecewise linear profile,

$$
\begin{aligned}
& \left(\frac{\partial \psi}{\partial x}\right)_{e}=\frac{\left(\psi_{E}-\psi_{P}\right)}{(\delta x)_{e}} \\
& \left(\frac{\partial \psi}{\partial x}\right)_{w}=\frac{\left(\psi_{P}-\psi_{W}\right)}{(\delta x)_{w}} \\
& \left(\frac{\partial \psi}{\partial y}\right)_{n}=\frac{\left(\psi_{N}-\psi_{P}\right)}{(\delta y)_{n}}, \\
& \left(\frac{\partial \psi}{\partial y}\right)_{s}=\frac{\left(\psi_{P}-\psi_{S}\right)}{(\delta y)_{s}} .
\end{aligned}
$$

Here $(\delta x)_{e},(\delta x)_{w},(\delta y)_{n}$, and $(\delta y)_{s}$ represent the distances between the grid point $P$ and the grid points $E, W, N$, and $S$, respectively (Figure 3.3). Substituting equation (3.46) in (3.45) and simplifying, one has,

$$
C_{E}\left(\psi_{E}-\psi_{P}\right)-C_{W}\left(\psi_{P}-\psi_{W}\right)+C_{N}\left(\psi_{N}-\psi_{P}\right)-C_{S}\left(\psi_{P}-\psi_{S}\right)-\omega_{P} \Delta x \Delta y=0
$$


where $\omega_{P}$ is the value of vorticity at the grid point $P$. Here it has been assumed that the value of $\omega$ at grid point $P$ prevails over the entire control volume. Thus $\bar{\omega}$ can be substituted with $\omega_{P}$. Also, the coefficients $C_{E}, C_{W}, C_{N}$ and $C_{S}$ are,

$$
\begin{gathered}
C_{E}=\frac{\Delta y}{(\delta x)_{e}}, \\
C_{W}=\frac{\Delta y}{(\delta x)_{w}}, \\
C_{N}=\frac{\Delta x}{(\delta y)_{n}}, \\
C_{S}=\frac{\Delta x}{(\delta y)_{s}} .
\end{gathered}
$$

Finally, the discretized equation for the grid point $P$ can be written,

$$
C_{P} \psi_{P}-\left(C_{E} \psi_{E}+C_{W} \psi_{W}+C_{N} \psi_{N}+C_{S} \psi_{S}\right)=-\omega_{P} \Delta x \Delta y
$$

where

$$
C_{P}=C_{E}+C_{W}+C_{N}+C_{S}
$$

The discretized equation given by equation (3.49) can be written for every grid point in the computational domain thus producing a set of simultaneous linear algebraic equations, the solution of which will give the value of the stream function at every grid point. The solution of a large set of algebraic equations, however, introduces additional challenges with respect to convergence characteristics and computational cost which must be considered carefully. These issues will be discussed later in this chapter.

\subsubsection{Vorticity transport and energy equations}

The discretization of the vorticity transport and energy equations will now be considered. Both these equations are of the advection-diffusion type. Such equations have mixed characteristics, and their mathematical classification changes with a change in the magnitude of the velocity vector, and in a more general formulation, the Peclet number, $P e$. At low values of $P e$, these equations have predominantly elliptic characteristics, whereas at high $P e$ they are primarily hyperbolic in nature. As such, special consideration must be given to the solution of these equations to ensure that the resulting solutions are valid over a wide range of Peclet numbers. This is achieved by treating the advection and diffusion parts

of the equation separately using different techniques. To illustrate, consider the following generic advection-diffusion equation, 
a)

\begin{tabular}{|l|l|l|l|l|l|l|l|}
\hline$\bullet$ & $\bullet$ & $\bullet$ & $\bullet$ & $\bullet$ & $\bullet$ & $\bullet$ & $\bullet$ \\
\hline$\bullet$ & $\bullet$ & $\bullet$ & $\bullet$ & $\bullet$ & $\bullet$ & $\bullet$ & $\bullet$ \\
\hline$\bullet$ & $\bullet$ & $\bullet$ & $\bullet$ & $\bullet$ & $\bullet$ & $\bullet$ & $\bullet$ \\
\hline$\bullet$ & $\bullet$ & $\bullet$ & $\bullet$ & $\bullet$ & $\bullet$ & $\bullet$ & $\bullet$ \\
\hline$\bullet$ & $\bullet$ & $\bullet$ & $\bullet$ & $\bullet$ & $\bullet$ & $\bullet$ & $\bullet$ \\
\hline
\end{tabular}

NN

b)

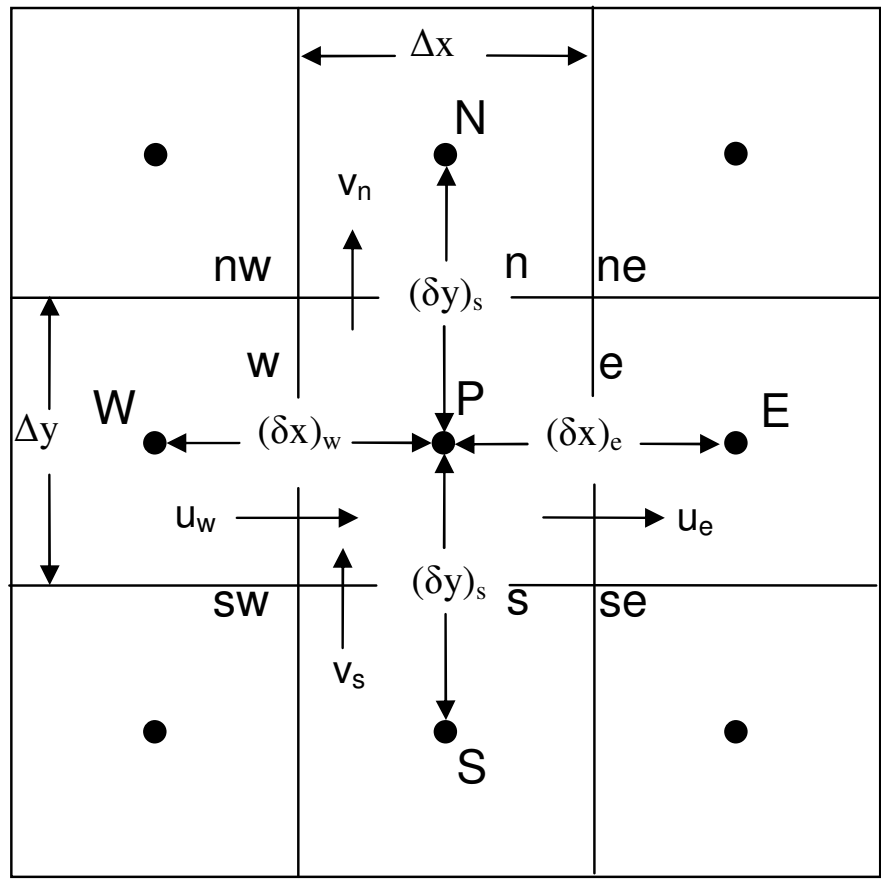

- EE
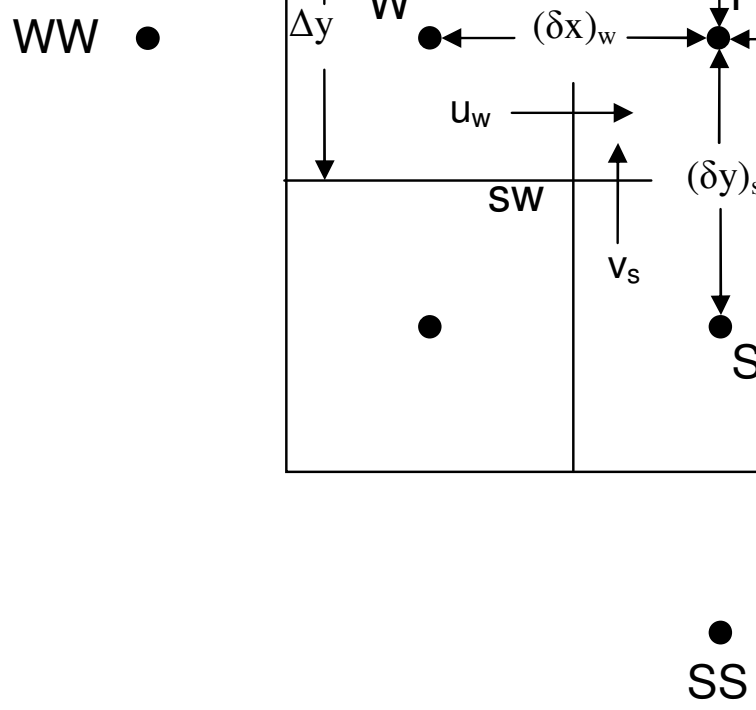

Figure 3.3: Schematic of (a) the computational grid, and (b) a single control volume. 


$$
\frac{\partial \theta}{\partial t}+(\mathbf{u} \cdot \nabla) \theta=\Gamma \nabla^{2} \theta+S
$$

Here $\theta$ represents either vorticity or temperature, $\Gamma$ denotes the diffusion coefficient, and $S$ represents the source-term. For the vorticity transport equation, $\Gamma=\operatorname{Pr}$, and the source term is,

$$
\begin{aligned}
S & =-\left(\frac{\operatorname{Pr} \phi^{2}}{D a}+\frac{F \phi^{2}}{\sqrt{D a}}|\mathbf{u}|\right) \omega+\frac{F \phi^{2}}{\sqrt{D a}}\left(u \frac{\partial|\mathbf{u}|}{\partial y}-v \frac{\partial|\mathbf{u}|}{\partial x}\right)+\operatorname{RaPr} \phi^{2} \frac{\partial T}{\partial x} \\
& =S_{C N}+S_{P} \omega .
\end{aligned}
$$

Here, $S_{C N}$ represents the constant part of the source terms and $S_{P}$ is the coefficient of $\omega$. Writing the source-term as the sum of $S_{C N}$ and $S_{P}$ linearizes it and incorporates it into the overall linear framework. The above linearization is in accordance with the procedure for source-term linearization in the control volume formulation [74]. For the energy equation, $\Gamma=\kappa$ and $S=0$. In conservative form, equation (3.51) can be written,

$$
\frac{\partial \theta}{\partial t}+\frac{\partial}{\partial x}(u \theta)+\frac{\partial}{\partial y}(v \theta)=\frac{\partial}{\partial x}\left(\Gamma \frac{\partial \theta}{\partial x}\right)+\frac{\partial}{\partial y}\left(\Gamma \frac{\partial \theta}{\partial y}\right)+S
$$

The above equation is discretized by integrating it over a single control volume and over the time interval $t$ to $t+\Delta t$,

$$
\begin{aligned}
\int_{s}^{n} \int_{w}^{e} \int_{t}^{t+\Delta t}\left[\frac{\partial \theta}{\partial t}+\right. & \left.\frac{\partial}{\partial x}(u \theta)+\frac{\partial}{\partial y}(v \theta)\right] \mathrm{d} t \mathrm{~d} x \mathrm{~d} y \\
& =\int_{s}^{n} \int_{w}^{e} \int_{t}^{t+\Delta t}\left[\frac{\partial}{\partial x}\left(\Gamma \frac{\partial \theta}{\partial x}\right)+\frac{\partial}{\partial y}\left(\Gamma \frac{\partial \theta}{\partial y}\right)+S\right] \mathrm{d} t \mathrm{~d} x \mathrm{~d} y
\end{aligned}
$$

To simplify the above equation, each side of the equation will be considered separately. For the right hand side (RHS) of equation (3.54), integration over the control volume gives,

$$
\begin{aligned}
\operatorname{RHS}=\int_{t}^{t+\Delta t}\left[\left\{\left(\Gamma \frac{\partial \theta}{\partial x}\right)_{e}-\left(\Gamma \frac{\partial \theta}{\partial x}\right)_{w}\right\} \Delta y+\left\{\left(\Gamma \frac{\partial \theta}{\partial y}\right)_{n}-\left(\Gamma \frac{\partial \theta}{\partial y}\right)_{s}\right\} \Delta x\right. \\
\left.+\left(S_{C N}+S_{P} \theta\right) \Delta x \Delta y\right] \mathrm{d} t
\end{aligned}
$$

As before, profile assumptions describing the variation of $\theta$ between the grid points are required in order to evaluate the derivatives in equation (3.55). Similar to the stream function, a piecewise linear profile is assumed for $\theta$, 


$$
\begin{aligned}
& \left(\Gamma \frac{\partial \theta}{\partial x}\right)_{e}=\frac{\Gamma_{e}\left(\theta_{E}-\theta_{P}\right)}{(\delta x)_{e}} \\
& \left(\Gamma \frac{\partial \theta}{\partial x}\right)_{w}=\frac{\Gamma_{w}\left(\theta_{P}-\theta_{W}\right)}{(\delta x)_{w}} \\
& \left(\Gamma \frac{\partial \theta}{\partial y}\right)_{n}=\frac{\Gamma_{n}\left(\theta_{N}-\theta_{P}\right)}{(\delta y)_{n}} \\
& \left(\Gamma \frac{\partial \theta}{\partial y}\right)_{s}=\frac{\Gamma_{s}\left(\theta_{P}-\theta_{S}\right)}{(\delta y)_{s}}
\end{aligned}
$$

Here $\Gamma_{e}, \Gamma_{w}, \Gamma_{n}$ and $\Gamma_{s}$ denote the diffusion coefficients at the control volume faces $e, w$, $n$, and $s$ respectively. Substituting equation (3.56) in (3.55) one obtains,

$$
\begin{aligned}
\text { RHS }=\int_{t}^{t+\Delta t}[ & \left\{\frac{\Gamma_{e}\left(\theta_{E}-\theta_{P}\right)}{(\delta x)_{e}}-\frac{\Gamma_{w}\left(\theta_{P}-\theta_{W}\right)}{(\delta x)_{w}}\right\} \Delta y \\
& \left.+\left\{\frac{\Gamma_{n}\left(\theta_{N}-\theta_{P}\right)}{(\delta y)_{n}}-\frac{\Gamma_{s}\left(\theta_{P}-\theta_{S}\right)}{(\delta y)_{s}}\right\} \Delta x+\left(S_{C N}+S_{P} \theta\right) \Delta x \Delta y\right] \mathrm{d} t .
\end{aligned}
$$

One now needs to complete the integration over the time-step, which can be done using several different methods. In the present study a fully implicit scheme time integration scheme will be used. The advantage of this technique is that it is unconditionally stable and ensures that physically realistic solutions are always obtained. The time integration in this scheme is given as,

$$
\int_{t}^{t+\Delta t} \theta \mathrm{d} t=\theta^{1} \Delta t
$$

where $\theta^{1}$ represents the value of $\theta$ during the current iteration. The fully implicit scheme therefore postulates that at any time $t$ the value of $\theta$ drops abruptly from its value during the previous iteration, $\theta^{0}$, to its current value and stays at that value for the rest of the time step. Thus the temperature during the time step is characterized by $\theta^{1}$. Using this scheme, equation (3.57) can be written,

$$
\begin{aligned}
\mathrm{RHS}= & {\left[\frac{\Gamma_{e}\left(\theta_{E}-\theta_{P}\right)}{(\delta x)_{e}}-\frac{\Gamma_{w}\left(\theta_{P}-\theta_{W}\right)}{(\delta x)_{w}}\right] \Delta y \Delta t } \\
& +\left[\frac{\Gamma_{n}\left(\theta_{N}-\theta_{P}\right)}{(\delta y)_{n}}-\frac{\Gamma_{s}\left(\theta_{P}-\theta_{S}\right)}{(\delta y)_{s}}\right] \Delta x \Delta t+\left(S_{C N}+S_{P} \theta\right) \Delta x \Delta y \Delta t
\end{aligned}
$$


where the superscript ' 1 ' has been dropped for convenience. Similarly the left hand side (LHS) of equation (3.54) can be written,

$$
\text { LHS }=\left(\theta_{P}-\theta_{P}^{0}\right) \Delta x \Delta y+\left(u_{e} \theta_{e}-u_{w} \theta_{w}\right) \Delta y \Delta t+\left(v_{n} \theta_{e}-v_{s} \theta_{w}\right) \Delta x \Delta t .
$$

Equating equations (3.59) and (3.60), the discretized form of the general transport equation can be written,

$$
\begin{aligned}
\left(\theta_{P}-\theta_{P}^{0}\right) \frac{\Delta x \Delta y}{\Delta t}+\left(u_{e} \theta_{e}-u_{w} \theta_{w}\right) \Delta y+\left(v_{n} \theta_{e}-v_{s} \theta_{w}\right) \Delta x \\
=\left[\frac{\Gamma_{e}\left(\theta_{E}-\theta_{P}\right)}{(\delta x)_{e}}-\frac{\Gamma_{w}\left(\theta_{P}-\theta_{W}\right)}{(\delta x)_{w}}\right] \Delta y+\left[\frac{\Gamma_{n}\left(\theta_{N}-\theta_{P}\right)}{(\delta y)_{n}}-\frac{\Gamma_{s}\left(\theta_{P}-\theta_{S}\right)}{(\delta y)_{s}}\right] \Delta x \\
+\left(S_{C N}+S_{P} \theta\right) \Delta x \Delta y .
\end{aligned}
$$

The above discretization can be further simplified to,

$$
\begin{aligned}
\left(\theta_{P}-\theta_{P}^{0}\right) a_{P}^{0}+\left(J_{e} \theta_{e}-J_{w} \theta_{w}\right)+\left(J_{e} \theta_{e}-J_{w} \theta_{w}\right)=D_{e}\left(\theta_{E}-\theta_{P}\right) & \\
& -D_{w}\left(\theta_{P}-\theta_{W}\right)+D_{n}\left(\theta_{N}-\theta_{P}\right)-D_{s}\left(\theta_{P}-\theta_{S}\right)+\left(S_{C N}+S_{P} \theta\right) \Delta x \Delta y
\end{aligned}
$$

where the time constant $a_{P}^{0}$ and the flow rates across the control volume faces, $J_{e}, J_{w}, J_{n}$, and $J_{s}$, are,

$$
\begin{gathered}
a_{P}^{0}=\frac{\Delta x \Delta y}{\Delta t} \\
J_{e}=u_{e} \Delta y, \\
J_{w}=u_{w} \Delta y, \\
J_{n}=v_{n} \Delta x, \\
J_{s}=v_{s} \Delta x .
\end{gathered}
$$

Also, the coefficients $D_{e}, D_{w}, D_{n}$, and $D_{s}$ are,

$$
\begin{gathered}
D_{e}=\frac{\Gamma_{e} \Delta y}{(\delta x)_{e}}, \\
D_{w}=\frac{\Gamma_{w} \Delta y}{(\delta x)_{w}}, \\
D_{n}=\frac{\Gamma_{n} \Delta x}{(\delta y)_{n}},
\end{gathered}
$$




$$
D_{s}=\frac{\Gamma_{s} \Delta x}{(\delta y)_{s}}
$$

Equation (3.62) reveals that the discretized equation contains the value of $\theta$ at the four control volume faces, which arises from the discretization of the convective part of the transport equation. In order to complete the discretization equation, an interpolation formula for the face values of $\theta$ in terms of its values at the neighboring grid points is required. This interpolation can be done using several different techniques. The two most commonly used methods are central differencing and upwind differencing. Both of these methods however suffer from severe limitations. The central difference scheme tends to be dispersive and produces oscillatory behavior in the obtained solution for strongly convective flows. Also, this scheme often leads to physically unrealistic solutions [81]. On the other hand, the upwind differencing scheme gives rise to false, or artificial, diffusion which tends to smear out sharp changes or gradients in the solution. In addition, the upwind scheme has a first-order truncation error. As such, excessively fine grids are needed in order to attain a grid independent solution.

The problems associated with both the central and upwind differencing schemes can be overcome by using a three-point upstream-weighted quadratic interpolation scheme for each wall value. This scheme, known as Quadratic Upwind Interpolation for Convective Kinetics, or QUICK, was first proposed by Leonard [75] and has since been tested against several model problems [76-78]. This scheme simultaneously possesses good accuracy and convective stability and has been found to be superior to other numerical schemes [79, 80]. It has also been applied to various engineering problems by different investigators and has been found to give highly accurate results. Owing to its many advantages, this scheme will be used in the present study.

\subsubsection{The QUICK scheme}

Since the original publication of the QUICK scheme, several modifications have been proposed for implementing the scheme for a variety of problems more complex than the one originally inspected by Leonard [75]. In this study a modified version of the QUICK scheme proposed by Hayase et al. [81] will be used. This scheme has been specifically developed within the framework of the control volume formulation and can handle problems involving unsteady convection with source terms. To illustrate the basic concepts of this scheme, consider equation (3.62). According to the QUICK scheme, the control volume face values of $\theta$ are, 


$$
\begin{gathered}
\theta_{e}= \begin{cases}\theta_{P}+S_{e}^{+}, & J_{e}>0, \\
\theta_{E}+S_{e}^{-}, & J_{e} \leq 0,\end{cases} \\
\theta_{w}= \begin{cases}\theta_{W}+S_{w}^{+}, & J_{w}>0, \\
\theta_{P}+S_{w}^{-}, & J_{w} \leq 0,\end{cases} \\
\theta_{n}= \begin{cases}\theta_{P}+S_{n}^{+}, & J_{n}>0, \\
\theta_{N}+S_{n}^{-}, & J_{n} \leq 0,\end{cases} \\
\theta_{s}= \begin{cases}\theta_{S}+S_{s}^{+}, & J_{s}>0, \\
\theta_{P}+S_{s}^{-}, & J_{s} \leq 0,\end{cases}
\end{gathered}
$$

where

$$
\begin{array}{cl}
S_{e}^{+}=\frac{1}{8}\left(3 \theta_{E}^{0}-2 \theta_{P}^{0}-\theta_{W}^{0}\right) & S_{e}^{-}=\frac{1}{8}\left(3 \theta_{P}^{0}-2 \theta_{E}^{0}-\theta_{E E}^{0}\right) \\
S_{w}^{+}=\frac{1}{8}\left(3 \theta_{P}^{0}-2 \theta_{W}^{0}-\theta_{W W}^{0}\right) & S_{e}^{-}=\frac{1}{8}\left(3 \theta_{W}^{0}-2 \theta_{P}^{0}-\theta_{E}^{0}\right) \\
S_{n}^{+}=\frac{1}{8}\left(3 \theta_{N}^{0}-2 \theta_{P}^{0}-\theta_{S}^{0}\right) & S_{e}^{-}=\frac{1}{8}\left(3 \theta_{P}^{0}-2 \theta_{N}^{0}-\theta_{N N}^{0}\right) \\
S_{s}^{+}=\frac{1}{8}\left(3 \theta_{P}^{0}-2 \theta_{S}^{0}-\theta_{S S}^{0}\right) & S_{e}^{-}=\frac{1}{8}\left(3 \theta_{S}^{0}-2 \theta_{P}^{0}-\theta_{N}^{0}\right)
\end{array}
$$

Here the superscript ' 0 ' indicates a value from the previous iteration and the grid points $E E, W W, N N$, and $S S$ are as shown in Figure 3.3. The QUICK scheme introduces a parabolic correction term (3.67a-d) to the standard upwind differencing scheme and can thus be regarded as a higher order upwind differencing scheme. The truncation error of the QUICK scheme is third-order, and it is thus often referred to as a third-order upwind scheme. The third-order truncation error significantly reduces the false diffusion that is inherent in the upwind scheme while retaining its convective stability.

The final discretization equation for the general transport equation can now be written. Substituting equations (3.66) and (3.67) into (3.62), and simplifying, the general discretization equation is,

$$
a_{P} \theta_{P}=a_{E} \theta_{E}+a_{W} \theta_{W}+a_{N} \theta_{N}+a_{S} \theta_{S}+b
$$

where

$$
a_{e}=D_{e}+\left\langle-J_{e}, 0\right\rangle,
$$




$$
\begin{gathered}
a_{w}=D_{w}+\left\langle J_{w}, 0\right\rangle, \\
a_{n}=D_{n}+\left\langle-J_{n}, 0\right\rangle, \\
a_{s}=D_{s}+\left\langle J_{s}, 0\right\rangle, \\
a_{P}=a_{E}+a_{W}+a_{N}+a_{S}+a_{P}^{0}-S_{P} \Delta x \Delta y, \\
b=a_{P}^{0} \theta_{P}^{0}+S_{C} \Delta x \Delta y+\left(S_{e}^{0}-S_{w}^{0}+S_{n}^{0}-S_{s}^{0}\right) .
\end{gathered}
$$

Here the operator $\langle X, Y\rangle$ denotes the greater of $X$ and $Y$. The terms $S_{e}^{0}, S_{w}^{0}, S_{n}^{0}$ and $S_{s}^{0}$ are the correction terms for the QUICK scheme which are treated as additional source terms. They are,

$$
\begin{gathered}
S_{e}^{0}=S_{e}^{+}\left\langle F_{e}, 0\right\rangle-S_{e}^{-}\left\langle-F_{e}, 0\right\rangle, \\
S_{w}^{0}=S_{w}^{+}\left\langle F_{w}, 0\right\rangle-S_{w}^{-}\left\langle-F_{w}, 0\right\rangle, \\
S_{n}^{0}=S_{n}^{+}\left\langle F_{n}, 0\right\rangle-S_{n}^{-}\left\langle-F_{n}, 0\right\rangle, \\
S_{s}^{0}=S_{s}^{+}\left\langle F_{s}, 0\right\rangle-S_{s}^{-}\left\langle-F_{s}, 0\right\rangle .
\end{gathered}
$$

Equation (3.68) represents the general form of the discretization equation for any advection-diffusion type of transport equation. As seen earlier, a discretization equation can be written for every grid point in the computational domain. The solution of the vorticity transport and energy equations therefore reduces to a solution of a set of algebraic equations.

\subsubsection{Calculation of the Velocities}

In order to solve the vorticity transport and energy equations, the velocities at the control volume faces are needed, and discretized equations for calculating the velocities are required. Recalling the definition of the velocities, it can be seen that the discretized form of equation (3.23) will give the required discretized equations. Therefore the velocities at the control volume faces can be written as,

$$
\begin{array}{ll}
u_{e}=\frac{\psi_{s e}-\psi_{n e}}{\Delta y}, & u_{w}=\frac{\psi_{s w}-\psi_{n w}}{\Delta y}, \\
v_{n}=\frac{\psi_{n e}-\psi_{n w}}{\Delta x}, & v_{s}=\frac{\psi_{s e}-\psi_{s w}}{\Delta x},
\end{array}
$$

where $u_{e}, u_{w}, u_{n}$, and $u_{s}$ are the velocities at the control volume faces $e, w, n$, and $s$, respectively, and $\psi_{n e}, \psi_{s e}, \psi_{n w}$, and $\psi_{s w}$ are the values of the stream function at the 
four control volume corners. In writing the above equations, the earlier assumption of a piecewise linear profile for $\psi$ has been used. From equations (3.73) and (3.74), it can be seen that the values of $\psi$ at the control volume corners are required to evaluate the velocities. However, because the value of $\psi$ is available only at the grid points, interpolation formulas for evaluating it at the control volume corners are needed. These interpolation formulas are,

$$
\begin{aligned}
& \psi_{n e}=\frac{1}{2}\left[\left(\frac{\psi_{P}+\psi_{E}}{2}\right)_{i}+\left(\frac{\psi_{P}+\psi_{E}}{2}\right)_{i-1}\right], \\
& \psi_{n w}=\frac{1}{2}\left[\left(\frac{\psi_{P}+\psi_{W}}{2}\right)_{i}+\left(\frac{\psi_{P}+\psi_{W}}{2}\right)_{i-1}\right], \\
& \psi_{s e}=\frac{1}{2}\left[\left(\frac{\psi_{P}+\psi_{E}}{2}\right)_{i}+\left(\frac{\psi_{P}+\psi_{E}}{2}\right)_{i+1}\right], \\
& \psi_{s w}=\frac{1}{2}\left[\left(\frac{\psi_{P}+\psi_{W}}{2}\right)_{i}+\left(\frac{\psi_{P}+\psi_{W}}{2}\right)_{i+1}\right] .
\end{aligned}
$$

where $i$ represents the $i^{\text {th }}$ row in the computational grid. Once again, the piecewise linear profile of $\psi$ has been used to obtain these interpolation formulas.

\subsubsection{Treatment of Boundary Conditions}

The discretization equations given for the stream function-vorticity, vorticity transport, and energy equations are only valid for grid points that are situated in the interior of the computational domain. Additional considerations must be given for the treatment of grid points that are located adjacent the system boundaries and the fluid-porous layer interface. These grid points will henceforth be referred to as boundary grid points. The discretization equations for the boundary grid points must be derived separately so as to correctly incorporate the appropriate boundary conditions. Of particular importance is the derivation of discretization equations for boundary grid points in the QUICK scheme. Also, special consideration must be given for incorporating the Neumann boundary conditions for the energy equation.

First, consider the boundary conditions for the stream function-vorticity equation. Since this equation does not have any convective terms, and is only subjected to Dirichlet boundary conditions, derivation of the discretization equations for the boundary grid points is straightforward. Recalling the discretization equation (3.49), it can be seen that depending on which boundary is considered, either $\psi_{E}, \psi_{E}, \psi_{E}$, or $\psi_{E}$ will be known. For example, on the left boundary, $\psi_{W}$ is known, and the discretization equation can be written,

$$
C_{P} \psi_{P}-\left(C_{E} \psi_{E}+C_{N} \psi_{N}+C_{S} \psi_{S}\right)=-\omega_{P} \Delta x \Delta y
$$


where the boundary condition $\psi_{W}=0$ has been applied. This equation is applicable to the all left hand boundary grid points. Similar equations can be derived for grid points located at other boundaries.

The treatment of the boundary conditions for the vorticity transport equation will now be considered. Because this equation also has Dirichlet boundary conditions, the discretization equation for the boundary grid points can be written similar to that for the stream function-vorticity equation. For example, for a grid point on the left boundary of the system the discretization equation can be written,

$$
a_{P} \omega_{P}-\left(a_{B} \omega_{B}+a_{E} \theta_{E}+a_{N} \theta_{N}+a_{S} \theta_{S}\right)=b,
$$

where $\omega_{B}$ is the value of the vorticity at the boundary grid point $B$ (Figure 3.4) and the definitions of $a_{P}, a_{E}, a_{N}, a_{S}$, and $b$ are the same as before. Unlike the stream function equation however the value of $\omega_{B}$ is not given but instead must be calculated from the stream function-vorticity equation. For example, on the left boundary the vorticity is,

$$
\omega=\frac{\partial^{2} \psi}{\partial x^{2}}
$$

and $\omega_{B}$ must be evaluated from a finite-difference formula for the second-order stream function derivative. Several finite-difference formulations have been proposed for this purpose. In this study the formulation proposed by Orszag and Israeli [82] has been used as it has been found to very efficient for numerical solutions of the vorticity-stream function formulation of the Navier-Stokes equation [83]. According to this formulation $\omega_{B}$ is,

$$
\omega_{B}=\frac{10 \psi_{1, j}-\psi_{2, j}}{3 \Delta x^{2}}
$$

where $\psi_{1, j}$ and $\psi_{2, j}$ are the values of the stream function along and first and second vertical grid lines, respectively. In a similar manner, the vorticity values along the other boundaries can be calculated.

Another aspect that needs special consideration is the evaluation the parabolic correction terms for the QUICK scheme. Because the QUICK scheme involves a three-point quadratic interpolation, with two of the points taken from the upstream side, discretized equations for the boundary grid points cannot be written using the standard formulation as one will then need to consider points which lie outside the calculation domain. To illustrate consider a left boundary grid point as shown in Figure 3.4. From equation (3.67b) it can be seen that $S_{e}^{+}$cannot be calculated for this grid point as $\theta_{W}^{0}$ lies outside the domain. Similar observations can be made for grid points on other boundaries. To address this situation, different quadratic interpolation formulas are used for the boundary grid points, 


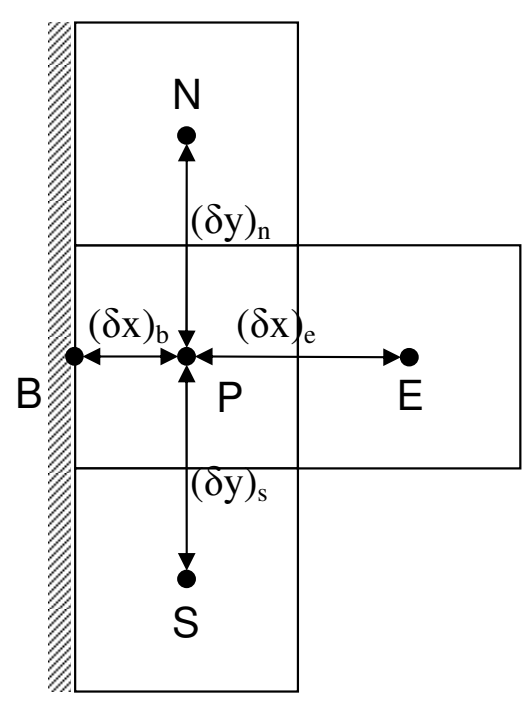

Figure 3.4: Boundary control volume on an adiabatic boundary.

$$
\begin{aligned}
& \text { Left Boundary : } S_{e}^{+}=\frac{1}{3}\left(\omega_{B}^{0}-\omega_{E}^{0}\right), \\
& \text { Right Boundary : } S_{w}^{-}=\frac{1}{3}\left(\omega_{W}^{0}-\omega_{B}^{0}\right), \\
& \text { Left Boundary : } S_{n}^{+}=\frac{1}{3}\left(\omega_{N}^{0}-\omega_{B}^{0}\right), \\
& \text { Left Boundary : } S_{s}^{-}=\frac{1}{3}\left(\omega_{S}^{0}-\omega_{B}^{0}\right),
\end{aligned}
$$

where $\omega_{B}^{0}$ is the boundary value of the vorticity. The equations for $S_{e}^{-}, S_{w}^{+}, S_{n}^{-}$and $S_{s}^{+}$are as given in equation (3.67).

Finally, the boundary conditions for the energy equation will be discussed. Since the temperature boundary condition at the top and bottom surfaces are of the Dirichlet type, the discretization equations for the top and bottom boundary grid points can be derived in the same manner as shown for the vorticity transport equation. Similarly the quadratic interpolation formulas for the boundary grid points will be identical to equation (3.80). Therefore, only the discretization equations for the Neumann boundary conditions at the left and right system boundaries are needed. To do this, consider once again a control volume on the left hand boundary of the system as shown in Figure 3.4. Because this boundary is adiabatic, the temperature needs to be evaluated at both the grid points $P$ 
and $B$. As such, discretization equations are needed for both these points. First, consider the grid point $P$. Since the left boundary is adiabatic and impermeable,

$$
\left(\Gamma \frac{\partial T}{\partial x}\right)_{w}=0, \quad u_{w}=0
$$

Following the discretization process shown in the previous section, one gets $D_{w}=0$ and $J_{w}=0$ which gives $a_{W}=0$. The discretization equation for $P$ can thus be written,

$$
\begin{gathered}
a_{P} \theta_{P}-\left(a_{E} \theta_{E}+a_{N} \theta_{N}+a_{S} \theta_{S}\right)=b, \\
a_{P}=a_{E}+a_{N}+a_{S}+a_{P}^{0} .
\end{gathered}
$$

where all the terms have their usual definitions. To derive a discretized equation for calculating the temperature at point $B$, equation $(3.56 \mathrm{~b})$ can be re-written for the boundary control volume shown in Figure (3.4) as,

$$
\left(\Gamma \frac{\partial T}{\partial x}\right)_{w}=\frac{\Gamma_{B}\left(T_{P}-T_{B}\right)}{(\delta x)_{B}}
$$

Because the boundary is adiabatic, the above equation gives $T_{B}=T_{P}$. Thus the temperature on the boundary is equal to the temperature at the grid point adjacent to the boundary. The discretized equations for control volumes on the right hand boundary can be obtained in a similar manner.

To complete the treatment of boundary conditions, the discretized equations for the grid points located adjacent to the fluid-porous layer interface will be discussed briefly. The discretized equation given by equation (3.68) is valid for the interfacial grid points. However, special consideration must be given to the diffusion coefficients at the interface. In order to derive the interfacial diffusion coefficients, the harmonic mean formulation of Patankar [74] will be used. According to this formulation, the interfacial diffusion coefficient, $\Gamma_{\text {int }}$, is,

$$
\Gamma_{\text {int }}=\frac{2 \Gamma_{\mathrm{f}} \Gamma_{\mathrm{m}}}{\Gamma_{\mathrm{f}}+\Gamma_{\mathrm{m}}}
$$

where $\Gamma_{\mathrm{f}}$ and $\Gamma_{\mathrm{m}}$ are the diffusion coefficients in the fluid and porous layers respectively. Thus for interfacial control volumes in the fluid layer, $\Gamma_{s}$ will be replaced by $\Gamma_{\text {int }}$, while for interfacial control volumes in the porous layer, $\Gamma_{n}$ will be replaced by $\Gamma_{\text {int }}$.

\subsubsection{Solution of the discretization equations}

The discretization process results in a set of linear algebraic equations of the form,

$$
M \Theta=b,
$$


where $M$ is a $N \times N$ matrix ( $N$ is the total number of grid points) and $\Theta$ is a vector of unknowns. An important characteristic feature of the matrix $M$ is that it is very sparse. Because the discretized equations have non-zero coefficients for only the neighboring grid points, only $5 \times N$ of the total $N^{2}$ entries in the matrix A are non-zero. In addition, the non-zero elements in the matrix have a specific fill pattern, a tri-diagonal structure with two additional diagonals on either side creating a symmetric penta-diagonal structure. Because matrix inversion is a computationally demanding task, it is important to consider linear solvers that can take advantage of this matrix structure.

In general, linear solvers can be classified as either direct or iterative. Direct methods typically do not take advantage of matrix sparseness and involve a fixed number of operations to obtain the final solution which is determined to machine accuracy. Also, because direct methods involve a large number of arithmetic operations, they involve high computational costs. Iterative methods on the other hand, can easily be formulated to take advantage of the matrix sparseness. Because these methods successively improve the solution, iterations can be stopped when the solution at any given iteration has been obtained to a sufficient level of accuracy and not have to incur the expense of obtaining the machine-accurate solution. Thus iterative solvers are most often used in computational fluid dynamics (CFD). Recently, however, much research has been devoted to the development of direct methods that take advantage of the matrix sparseness and also obtain solutions to machine accuracy. These solvers offer the advantages of both direct and iterative techniques and are thus highly attractive.

In this study, a state-of-the-art fast direct solver developed specifically for symmetric sparse matrices has been used [82]. This solver uses an up-looking Cholesky factorization for sparse matrices to obtain the final solution and can be readily implemented in the commercial software package MATLAB ${ }^{\circledR}$. In order to test the performance of this method, it is compared with two standard iterative techniques most commonly encountered in CFD studies: successive over relaxation (SOR) and preconditioned conjugate gradient (PCG). In each case tested, the direct method gives significantly lower solution times and better accuracy. For this reason, the direct method has been used for the simulations in the present study. A detailed description of the solution algorithm of this direct solver is given in Appendix A.

\subsubsection{General solution algorithm}

As seen previously, the numerical solution process basically involves the solution of three non-dimensional equations - the stream function-vorticity equation, the vorticity transport equation and the energy equation. Owing to the inherent coupling of these equations, as well as their strongly non-linear nature, an iterative solution process is employed. 
The solution is started from the given initial conditions and is marched forward in time through a series of successive iterations until steady-state is reached. This process thus emulates the temporal evolution of the flow and temperature fields from an initial state to steady-state. Every intermediate solution during the iterative process captures the transient state of the system at a particular instant. This final solution is the actual solution of the original non-linear problem that is obtained by the iterative solution of a set of linear equations. The solution process can be summarized as follows:

1. Initial values for the stream function, vorticity and temperature are assigned for all the grid points.

2. The convergence criterions are set and the iterative process is started.

3. Interpolation formulas are used to calculate the values of the stream function at the control volume corners. Using these values, the velocities at the control volume faces are calculated using (3.73) and (3.74).

4. The vorticity transport equation is then solved. For this, the discretization equation is recast into the form given by (3.84) and the direct method is invoked to solve the system of equations.

5. Using the obtained value of the vorticity, the stream function-vorticity equation is solved. As done for the vorticity equation, the discretization equation is expressed in matrix form and the resulting set of linear equations is solved using the direct method.

6. The energy equation is solved with a method similar to the vorticity transport equation.

7. Iterations are continued until steady state is reached.

\subsection{Post-processing of data}

Having described the numerical solution procedure for the present problem, the post-processing of the numerical data will be discussed briefly. It will be recalled that the aim of the numerical simulations is to understand the evolution of the flow and temperature fields and predict the overall heat transfer rates. Because the numerical solution gives a set of numeric values for the temperature, stream function and vorticity for the entire computational domain, graphical representation of the numerical data in terms of streamline and isotherm plots can be used to visualize and understand the flow patterns and temperature distribution. 
The overall heat transfer rate can be calculated by expressing it in dimensionless form using the conduction-referenced Nusselt number. The Nusselt number characterizes the non-dimensional temperature gradient, and it can be calculated at any point within the computational domain. In the present study, the average Nusselt number over the heated surface is determined by,

$$
\begin{aligned}
N u & =\frac{q}{k_{\mathrm{f}}\left(L_{H} / H\right)\left(T_{H}-T_{C}\right)} \\
& =\kappa\left(\frac{H}{L_{H}}\right) \int_{0}^{L_{H} / H}\left(\frac{\partial T}{\partial y}\right)_{y=0} \mathrm{~d} x .
\end{aligned}
$$

Here $q$ is the heat supplied to the system though the heater at the base and $L_{H}$ is the length of the heater. The temperature gradient is calculated using the first order accurate backward finite difference formula

$$
\left(\frac{\partial T}{\partial y}\right)_{y=0}=\frac{T_{H}-T_{1}}{(\delta x) / 2}
$$

where $T_{1}$ represents the non-dimensional temperatures along the first row of grid points situated just above the heater surface. It must be noted that higher order backward difference formulas in general give faster convergence to the grid independent value of the Nusselt number. However, in the present case it is seen that the first-order formula given in equation (3.86) has much better convergence characteristics. This is discussed in detail in Chapter 4 where the convergence characteristics of first and second order accurate finite difference formulas are compared. The integration in equation (3.85) is carried out using Simpson's rule.

\subsection{Convergence and accuracy of the numerical scheme}

\subsubsection{Convergence criterion}

For an iterative solution technique, there exists a residual error caused by the difference between the exact solution of the finite-difference equations, and the solution obtained when the iterations are stopped. Obviously, this error could be reduced to zero if the number of iterations were to be made very large. This is however not feasible in most practical scenarios due to the enormous computational costs that would be incurred. Thus it is essential to clearly define a convergence criterion in order to terminate the solution loop. The convergence criterion is usually expressed by: 1) calculating the total fractional change of each dependent variable in successive iterations, or 2) calculating the fractional change in the maximum value of each dependent variable in successive iterations. If this fractional 
change is less than a particular tolerance value, the solution is said to have converged. In the present study, the first formulation is adopted which can be expressed mathematically,

$$
\frac{\sum_{i=1}^{m} \sum_{j=1}^{n}\left|\vartheta_{i, j}^{n+1}-\vartheta_{i, j}^{n}\right|}{\sum_{i=1}^{m} \sum_{j=1}^{n}\left|\vartheta_{i, j}^{n+1}\right|}<\tau,
$$

where $\vartheta$ represents $\psi, \omega$ and $T, r$ represents the iteration number and $\tau$ is the tolerance limit. Also, $m$ and $n$ represent the number grid points in the $\mathrm{x}$ and $\mathrm{y}$ directions, respectively. The choice of the value tolerance limit is rather arbitrary. In the present study, the value of the tolerance limit is set at $10^{-5}$.

\subsubsection{Relaxation parameter}

Because of the strongly non-linear nature of the governing equations and the iterative process employed in the solution, the use of under relaxation parameters is required in order to ensure convergence. Under relaxation slows down the changes in the value of the dependent variable from iteration to iteration and prevents divergence in the solution. As

with other aspects of a numerical technique, there are no universal rules governing the selection of a particular method for introducing under relaxation or selecting a value for the relaxation parameter. As such exploratory simulations are required to determine the most efficient technique. After extensive trial runs, it was found that under relaxation of the stream function-vorticity equation alone is sufficient to ensure convergence in all cases. The procedure given by Patankar [74] is used for introducing the relaxation parameter. To briefly outline the procedure, equation (3.49) is re-written,

$$
\psi_{P}=\psi_{P}^{0}+\left(\frac{\sum_{i} C_{i} \psi_{i}-\omega_{P} \Delta x \Delta y}{C_{P}}-\psi_{P}^{0}\right)
$$

where the summation runs over the four adjacent grid point $E, W, N$ and $S$, and $\psi_{P}^{0}$ represents the value of the stream function from the previous iteration. From the above equation it can be seen the term within the parenthesis represents the change in the value of $\psi$ during the current iteration. This change can be modified by introducing a relaxation parameter $\Omega$, so that,

$$
\psi_{P}=\psi_{P}^{0}+\Omega\left(\frac{\sum_{i} C_{i} \psi_{i}-\omega_{P} \Delta x \Delta y}{C_{P}}-\psi_{P}^{0}\right) .
$$

Finally, the discretization equation with the relaxation parameter can be written, 


$$
\frac{C_{P}}{\Omega} \psi_{P}-\left(C_{E} \psi_{E}+C_{W} \psi_{W}+C_{N} \psi_{N}+C_{S} \psi_{S}\right)=-\omega_{P} \Delta x \Delta y+(1-\Omega) \frac{C_{P}}{\Omega} \psi_{P}^{0}
$$

It can be seen that when the iterations converge, i.e. when $\psi_{P}$ equals $\psi_{P}^{0}$, the original discretization equation is recovered. After several test runs, a value of 0.8 is selected for the relaxation parameter.

\subsubsection{Grid convergence}

The accuracy of the solutions depends strongly on the grid spacing. In general, an accurate solution is obtained only when the grid is sufficiently fine. However, a truly grid independent solution may be a difficult to obtain when the computational cost is taken into consideration. This is further compounded by the fact that grid convergence cannot be uniquely quantified as there are several parameters which can be used to provide an estimate for grid convergence. Also, grid independence is strongly dependent on the convergence criterion that is set for the iterative solution, as well as on different parameters, such as the Rayleigh number and the size of the heat source.

There are no universal rules for testing grid convergence. Thus exploratory calculations are required at the beginning of every computation to provide some useful information about the behavior of the solution scheme. From such computations, an appropriate grid size can be selected depending upon the value of the Rayleigh number, aspect ratio and heater size. In the present study, three different parameters are used to check for the grid convergence of the solution. These are the average Nusselt number along the heater surface, the maximum stream function, and the fractional energy difference between the top and bottom surfaces. Generally, grid refinement is continued until the percentage change in all these parameters is less than $1 \%$. The use of multiple parameters to quantify the grid convergence behavior of the solution increases the overall accuracy of the obtained results.

\subsubsection{Energy balance}

As mentioned above, an overall energy balance serves as a check on the accuracy of the results. The energy balance is verified by taking the fractional difference of the total energy input and output,

$$
\begin{aligned}
\Delta E & =\left[\frac{(\text { Total Heat Input })-(\text { Total Heat Output })}{(\text { Total Heat Input })}\right] \\
& =\left(\frac{N u_{b}-N u_{t}}{N u_{b}}\right)
\end{aligned}
$$

where $N u_{b}$ and $N u_{t}$ are the Nusselt number along the bottom and top surfaces and are 
defined as,

$$
\begin{aligned}
N u_{b} & =\frac{q_{b} H}{k_{\mathrm{f}} L\left(T_{H}-T_{C}\right)} \\
& =\kappa\left(\frac{H}{L}\right) \int_{0}^{L / H}\left(\frac{\partial T}{\partial y}\right)_{y=0} \mathrm{~d} x . \\
N u_{t} & =\frac{q_{t} H}{k_{\mathrm{f}} L\left(T_{H}-T_{C}\right)} \\
& =\left(\frac{H}{L}\right) \int_{0}^{L / H}\left(\frac{\partial T}{\partial y}\right)_{y=1} \mathrm{~d} x .
\end{aligned}
$$

For the exact solution, the value of $\Delta E$ is equal to zero because the finite difference equations have been derived using the control volume approach which, as mentioned earlier, possesses the characteristic of the integral conservation of energy. Therefore, from a theoretical point of view, the total heat output must be equal to the total heat input, thus making the value of $\Delta E$ zero. However, due to the accumulation of round-off errors, the value of $\Delta E$ is not zero but a very small number. This value depends on several parameters, such as the Rayleigh number, aspect ratio, the Darcy number and the size of the heat source. 


\section{Chapter 4}

\section{Numerical Results}

In this chapter, results from the numerical simulations are presented. As mentioned earlier, the aim of the numerical simulations is to predict the overall heat transfer coefficients, and understand the evolution of the flow and temperature fields in fluid-superposed porous layers heated locally from below. To that end, results from both steady-state and transient numerical solutions are presented here. First, the stability and convergence characteristics of the numerical solution are discussed. Thereafter, the validity of the numerical solution technique is demonstrated by comparing selected numerical results to prior studies on natural convection in porous layers, fluid layers, and fluid-superposed porous layers. The steady-state heat transfer characteristics of the system under consideration are then studied in detail. In particular, the effects of the various non-dimensional controlling parameters, such as the heater-to-base length ratio, the porous-to-fluid layer height ratio, the overall aspect ratio, the Darcy number, the solid-to-fluid conductivity ratio, and the fluid Prandtl number are investigated. Finally, the temporal evolution of the convective flow is examined and results are compared to the transient behavior of composite systems heated uniformly from below.

\subsection{Convergence, accuracy and stability of the numerical scheme}

The first step in determining the validity of the numerical solution technique is to test the convergence, accuracy and stability of the scheme. In the present study, the convergence of the numerical scheme is determined by evaluating the average Nusselt number along the heater surface and the maximum absolute value of the stream function, $\left|\psi_{\max }\right|$, for successively finer grids and calculating the percentage change in both these quantities with grid refinement. The solution is considered to be grid independent when any further change in grid size produces a less than one percent change in $N u$ and $\left|\psi_{\max }\right|$. The parameters used in the simulations for testing grid convergence are: $\delta=0.5, \eta=0.5, A=2, D a=$ 
(a)

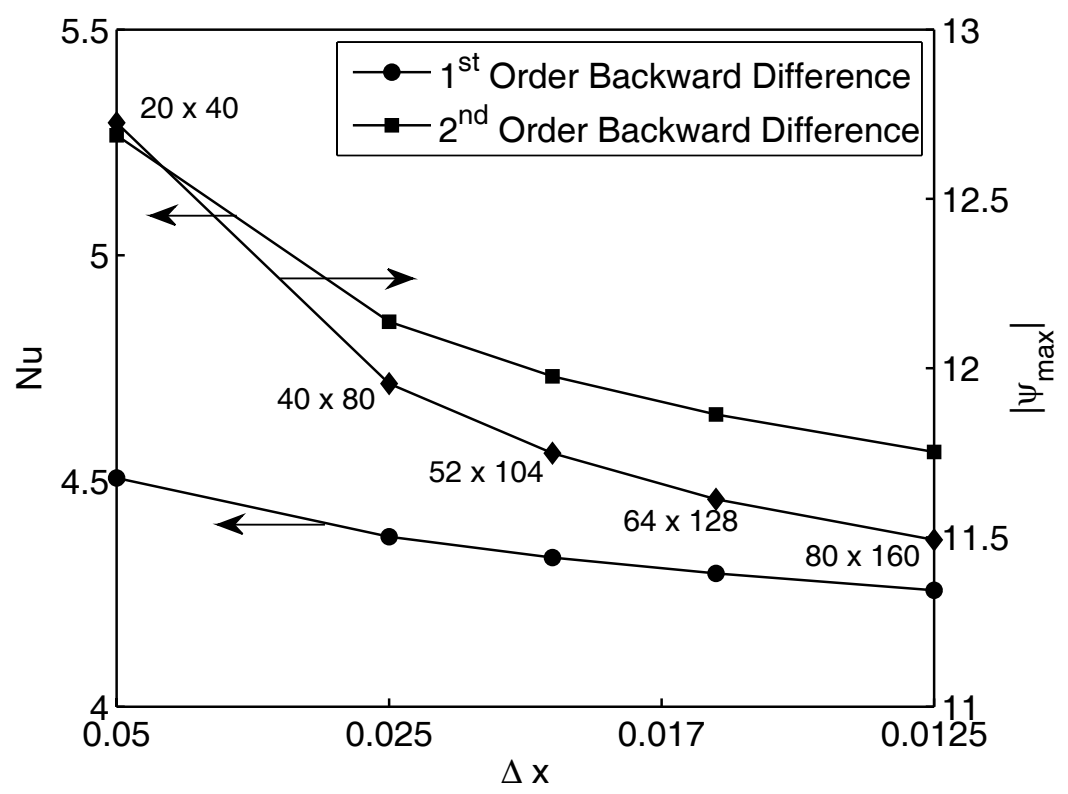

(b)

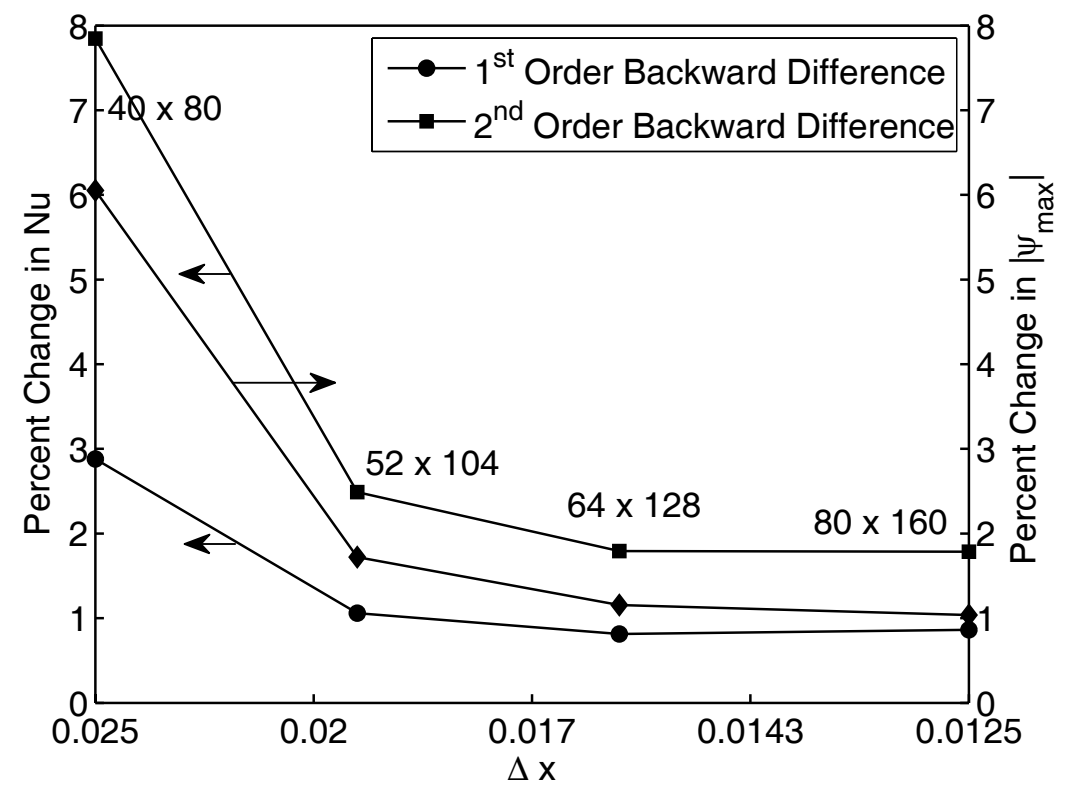

Figure 4.1: Convergence characteristics of the numerical scheme. (a) Change in average Nusselt number, $N u$ and maximum value of the stream function, $\left|\psi_{\max }\right|$ with grid refinement. (b) Percentage change in $N u$ and $\left|\psi_{\max }\right|$ with successive grid refinement. 


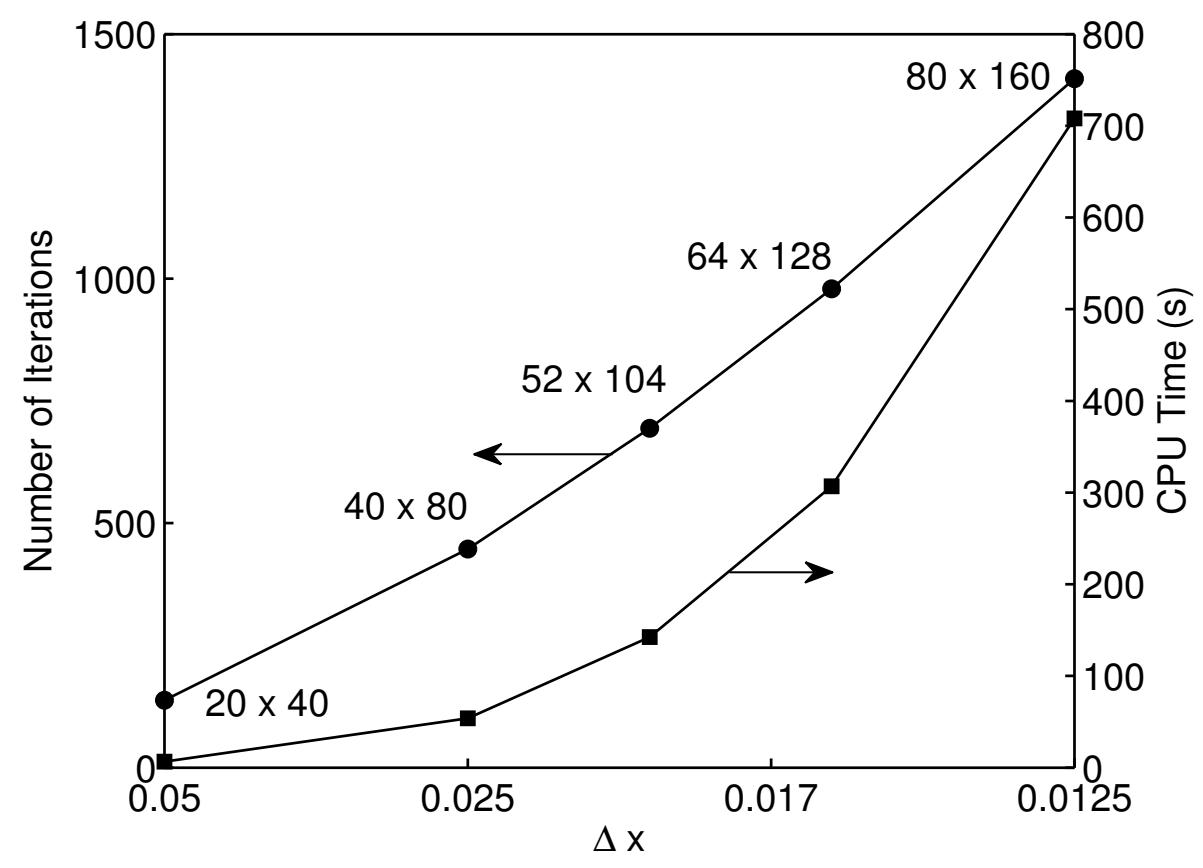

Figure 4.2: Grid convergence of the solution in terms of the number of iterations and the CPU time.

$10^{-6}, \operatorname{Pr}=7, \lambda=1$, and $R a=10^{5}$, where all the symbols have their usual definitions. In addition, for all the simulations reported in this chapter, the following parameters are kept fixed: $\phi=0.4, F=0.5$, and $\Omega$ (relaxation parameter) $=0.8$.

The grid convergence behavior of the solution is shown graphically in Figure 4.1. In addition, the values of the Nusselt number evaluated from both first and second-order backward difference formulas for the temperature gradient at the heater surface are shown. As can be seen, the Nusselt number calculated using a first-order finite difference scheme converges more rapidly to the final value than the second-order scheme. The reason for this is that for localalized heat sources, the temperature gradients along the heater edges are very high and heat flow there occurs in both the normal and lateral directions. Hence using higher-order finite difference schemes to calculate the normal temperature gradient at the heater surface requires an excessively fine grid. It was found that a grid size of $120 \times 240$ is required to achieve similar results using both first and second-order finite difference schemes. Using a first-order scheme, however, the solution converges at grid size of $52 \times 104$. This is further confirmed by noting that further grid refinement produces only about a one percent change in the value of $|\psi|_{\max }$. This trend is noticed in all the solutions reported later in this chapter. For this reason, a first-order backward difference scheme is selected for calculating the Nusselt number in all subsequent results. It can also be seen that for grids coarser than $40 \times 80$, grid refinement causes a much larger percentage change in $N u$ and $|\psi|_{\max }$ 


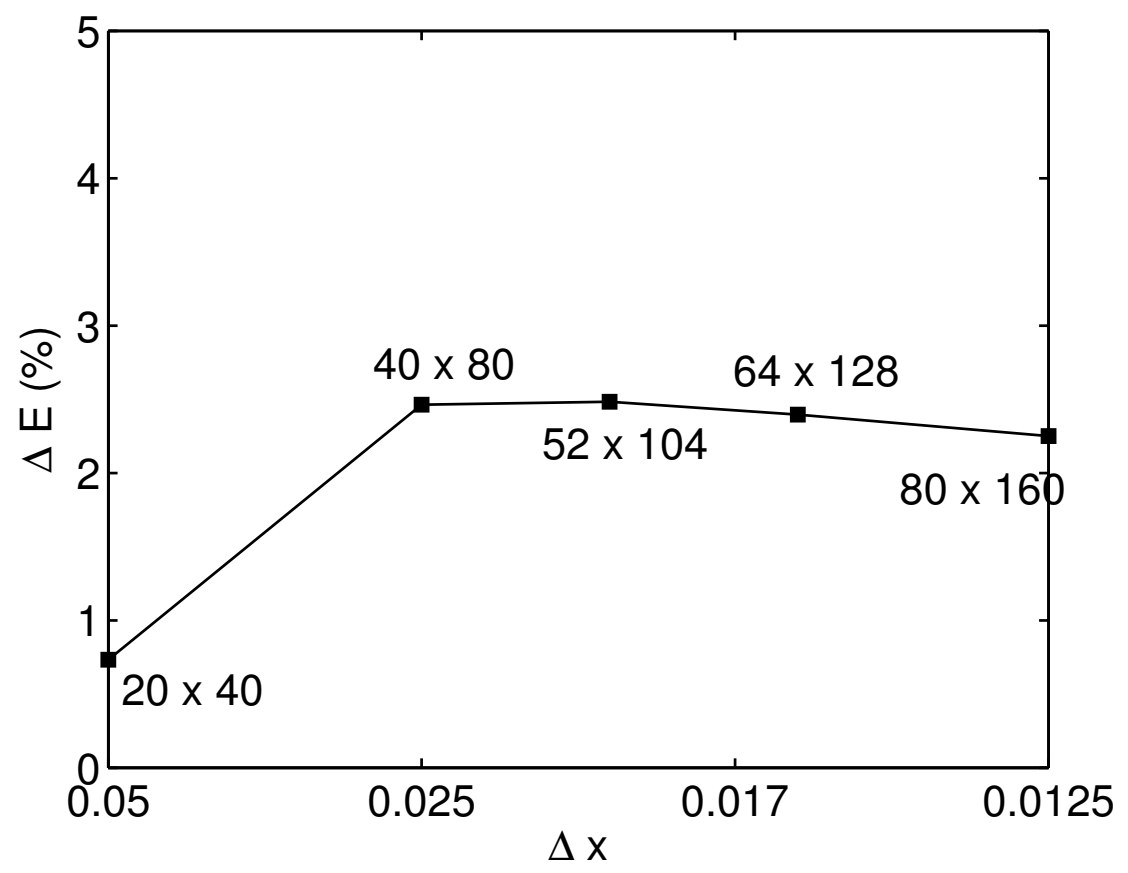

Figure 4.3: Energy balance given in terms of the percentage difference between the heat fluxes at the top and bottom surfaces.

than any successive grid refinement. As such, this is the minimum grid size selected for all calculations. Figure 4.2 shows the convergence behavior of the solution in terms of the number of iterations and CPU time.

As a further check on the accuracy of the solutions, the energy balance is calculated for all solutions. The energy balance for the test problem is shown in Figure 4.3. It can be seen that the heat fluxes at the top and bottom surfaces differ by about $2.5 \%$ when the solution converges. This difference is somewhat higher when compared to problems with uniform heating along the base where the energy balance is always less than one percent at similar grid sizes, all other parameters being kept constant. This is again, most likely due to the strong lateral temperature gradients near the heater edges. It is probable that a nonuniform grid with a high number of control volumes along the base may more accurately capture the temperature gradients and give a better energy balance. However, with the energy balance less than $2.5 \%$ for most of the cases run in the present study, a non-uniform grid was not considered so as to put all of the results on an equal basis.

Finally, it is also worthwhile to look at the stability of the numerical solution technique. As mentioned in the previous chapter, a fully implicit time integration scheme is adopted in the present study. This scheme is unconditionally stable, and therefore no oscillations are expected in the Nusselt number during the progress of the solution towards steady-state. Figure 4.4, which shows the time evolution of the Nusselt number, confirms 


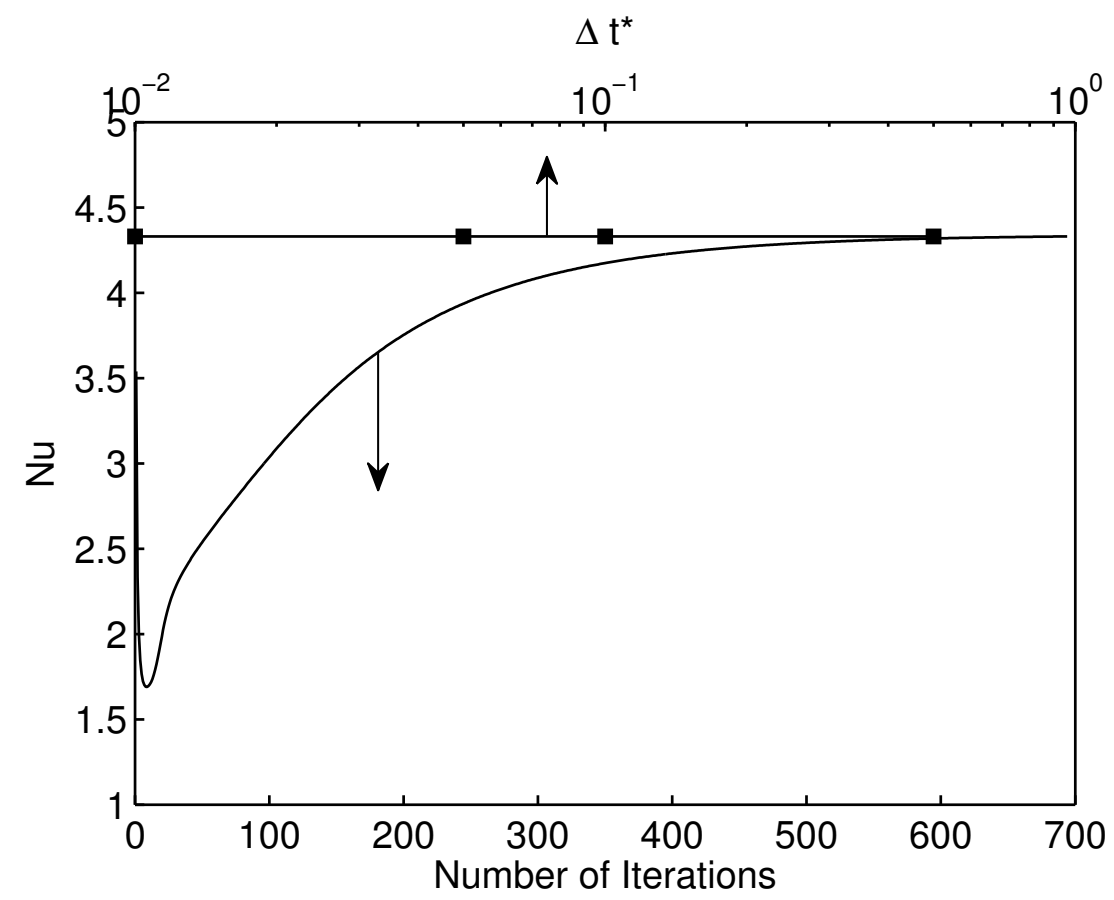

Figure 4.4: Time evolution of the Nusselt number the effect of the time step.

this expectation. In addition, it can be seen that the time step selected does not have any effect on the final solution, as is to be expected for a fully implicit scheme. For this reason, a time step of 0.1 is selected for all computations as it gives good balance between accuracy and computational time.

\subsection{Verification of the numerical scheme}

In order to verify the numerical solution technique, numerical results are first obtained for three base problems: natural convection in a fluid layer, natural convection in a saturated porous layer, and natural convection in a fluid-superposed porous layer, all heated uniformly from below. The first problem is the well known Rayleigh-Bénard problem, while the second problem is the equivalent of the Rayleigh-Bénard problem for porous media, also known in the literature as the Horton-Rogers-Lapwood problem. Both these problems have been extensively studied, and well established solutions for them are available in the literature. These two problems are therefore ideal for testing the accuracy of the numerical scheme. In addition, numerical results are also compared to past studies on convection in fluid-superposed porous layers, although it must be noted that there are no standard benchmark studies available in the literature for this problem. The various parameters used in these simulations are given in Table 4.1. 
Table 4.1: Parameters used in the simulations for code validation.

\begin{tabular}{|l|l|l|l|l|}
\hline Base Problem & $\begin{array}{l}\text { Parameters } \\
\text { Studied }\end{array}$ & $\begin{array}{l}\text { Range of } \\
\text { Values }\end{array}$ & $\begin{array}{l}\text { Fixed } \\
\text { Parameters }\end{array}$ & $\begin{array}{l}\text { Data } \\
\text { Presented }\end{array}$ \\
\hline \hline $\begin{array}{l}\text { 1. Rayleigh-Benard } \\
\text { Problem }\end{array}$ & $R a, N u$ & $R a=10^{3}-10^{5}$ & $\begin{array}{l}A=2 \\
P r=7\end{array}$ & $N u$-vs- $R a$ \\
\hline $\begin{array}{l}\text { 2. Horton-Rogers- } \\
\text { Lapwood Problem }\end{array}$ & $R a_{\mathrm{m}}, N u_{\mathrm{m}}$ & $R a_{\mathrm{m}}=10-350$ & $\begin{array}{l}A=2 \\
P r=7\end{array}$ & $N u_{\mathrm{m} \text {-vs- } R a_{\mathrm{m}}}$ \\
& & & $\begin{array}{l}\mathrm{Da}=10^{-6} \\
\lambda=1\end{array}$ & \\
\hline $\begin{array}{l}\text { 3. Fluid-Superposed } \\
\text { Porous Layer } \\
\text { Problem }\end{array}$ & $R a, N u$ & $R a=10^{3}-10^{5}$ & $\begin{array}{l}A=2 \\
P r=7\end{array}$ & $N u$-vs- $R a$ \\
& & & $D a=10^{-6}$ & \\
\hline
\end{tabular}

The first test of the numerical solution technique is to compare results for the Rayleigh-Bénard problem. Recalling the definition of the height ratio, $\eta$, and the heater length, $\delta$, it can be seen that this problem is a special case of the present problem for $\eta=0$ and $\delta=1$. Figure 4.5 shows the comparison of the present numerical results with the numerical solutions of Clever and Busse [85], and Ouertatani et al. [86], as well as with experimental results from various investigators compiled by Hollands et al. [87]. As can be seen, the current numerical results are slightly lower than those of Clever and Busse [85] and slightly higher than benchmark numerical solutions of Ouertatani et al. [86]. The maximum difference, however, is less than seven percent in either case. The numerical results also show excellent agreement with the experimental data compiled by Hollands et al. [87], especially at high Rayleigh numbers. The present numerical code slightly under predicts the onset of convection, and the Nusselt numbers near the onset point. The critical Rayleigh number is found to be $\sim 1800$ whereas the theoretical value for the critical Rayleigh number is 1708 . This discrepancy is most likely due to the effect of the lateral walls on convective motion. As Catton has pointed out $[68,69]$, lateral walls introduce additional shear, which delays the onset of convection and subsequent convective motion. Because the domain studied here has a relatively short horizontal extent $(L / H=2)$, the effect of the lateral walls is significant at low Rayleigh numbers.

Comparison to literature results for the Horton-Rogers-Lapwood problem is shown in Figures $4.6(\mathrm{a}, \mathrm{b})$. This problem is a special case of the current problem for $\eta=1$ and $\delta=1$. Figure 4.6(a) shows the comparison with the numerical results of Caltagirone [88] 


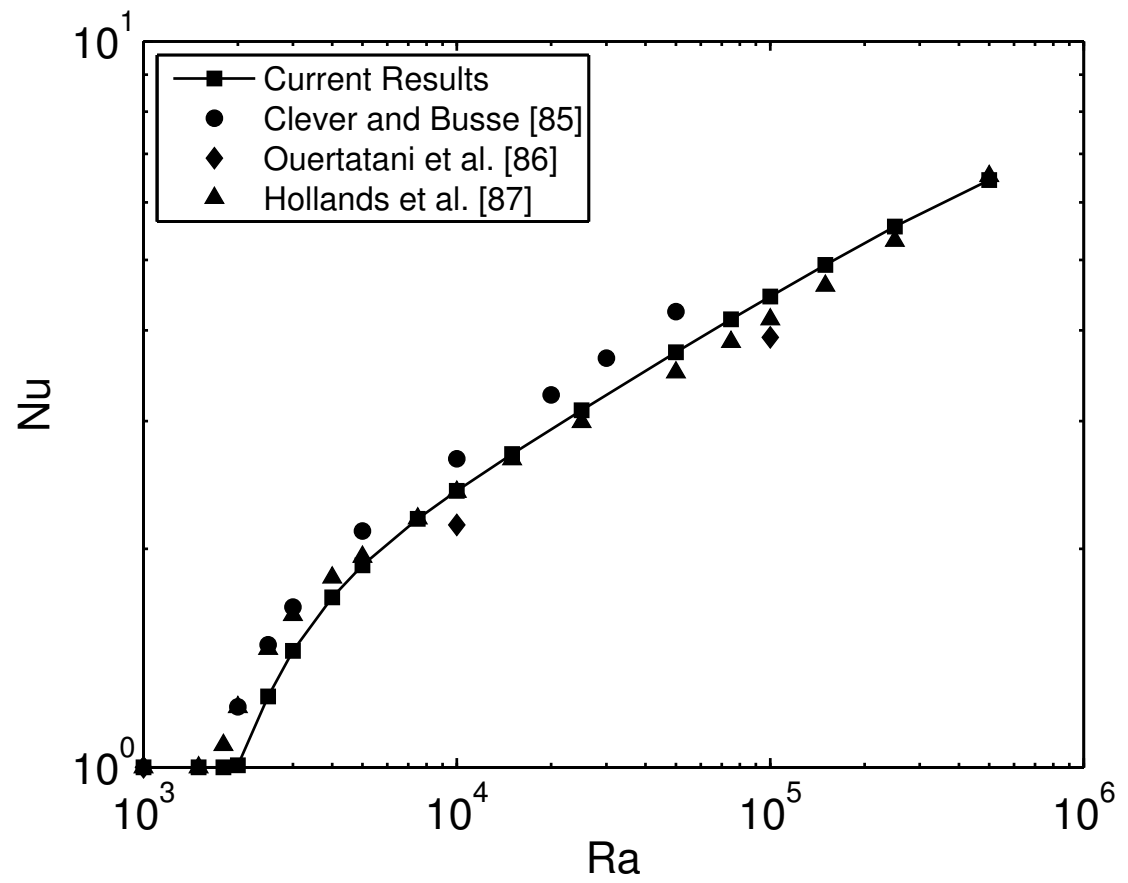

Figure 4.5: Nusselt-versus-Rayleigh number relations for the Rayleigh-Bénard problem showing comparison with literature data.

and Combarnous and Bories [4]. It can be seen that the present numerical results show excellent agreement with these studies. The critical Rayleigh number $\left(R a_{\mathrm{m}}\right)$ for the onset of convection predicted by the present results is 40 , which is very close to the theoretical value of 39.48. It is well known that for convection in saturated porous layers, there exists a second critical point beyond which oscillatory convection prevails and no steady-state is attained. The value of this second critical Rayleigh number is approximately in the range $380-390$. In the present study, no attempt was made to evaluate this second critical Rayleigh number although it must be noted that no unique steady-state solution was obtained for $R a_{\mathrm{m}}>400$. Figure 4.5 also shows excellent agreement of the present numerical results with the experimental results of Elder [29], Buretta and Berman [38], and Combarnous and Bories [4] except at very high $R a_{\mathrm{m}}$ where the code slightly under predicts the Nusselt number. This trend has been reported in earlier numerical studies [4]. Combarnous and Bories have suggested that this may be due to the effects of the variation of material properties with temperature as well as due to the breakdown of the assumption of thermal equilibrium between the solid and fluid phases, both of which can become pronounced at high Rayleigh numbers.

Finally, numerical results for the problem of convection in fluid-superposed porous layers heated uniformly from below are compared to literature data. Figures 4.7 (a,b) show the comparison of the present numerical results to the numerical results of Poulikakos 
(a)

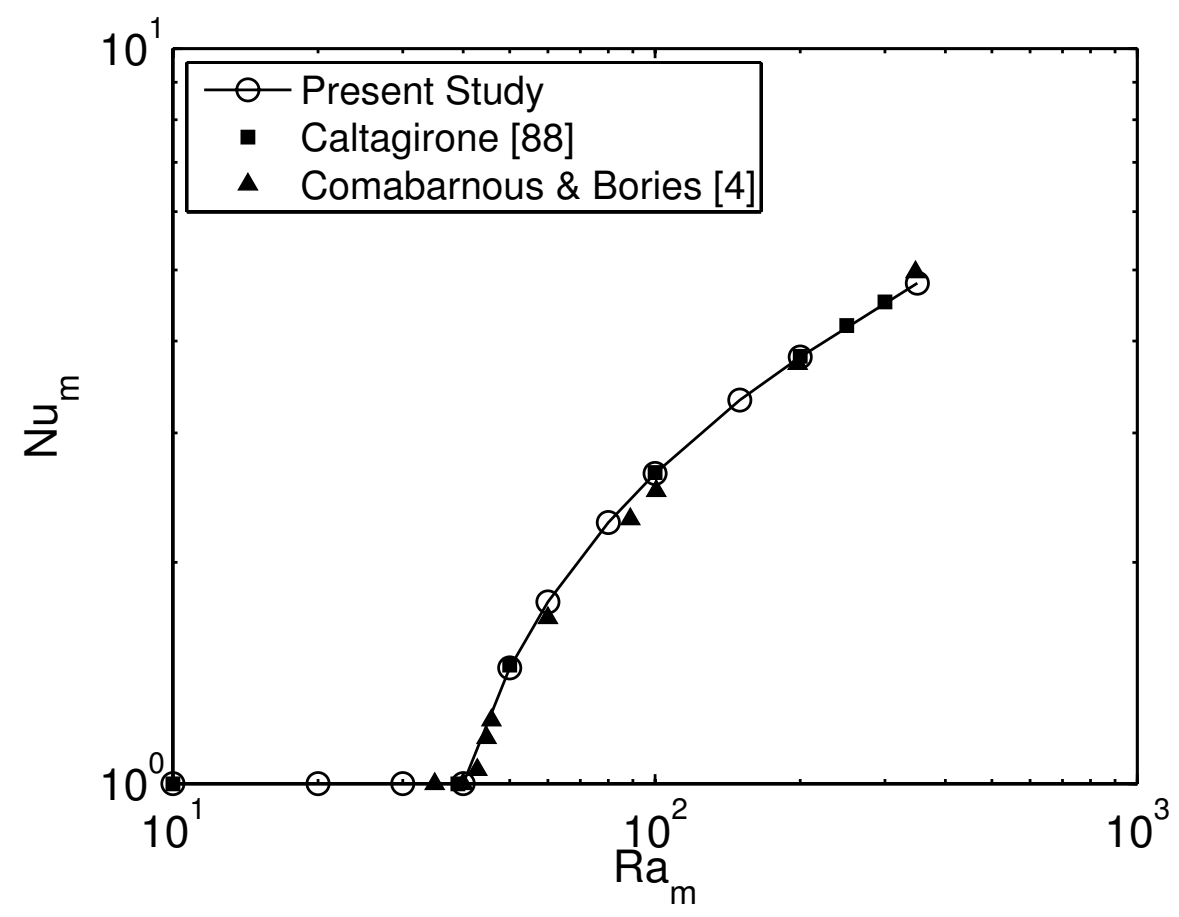

(b)

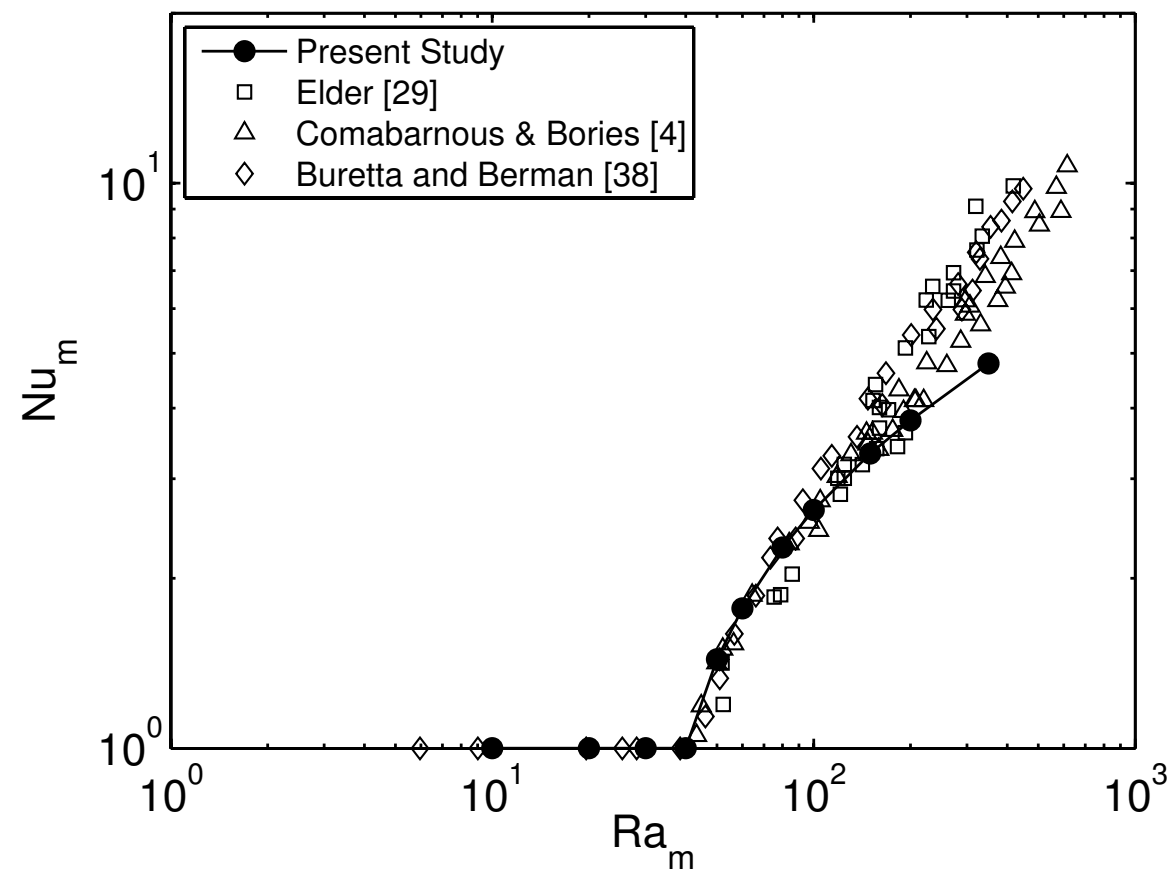

Figure 4.6: Nusselt-versus-Rayleigh number relations for the Horton-Rogers-Lapwood problem. (a) Comparison to numerical studies. (b) Comparison to experimental data. 
[20] and Poulikakos et al. [21]. It can be seen that the present numerical results agree reasonably well with the results of Poulikakos [20]. The present method predicts a slightly lower critical Rayleigh number, and also over predicts the Nusselt number near the onset point, compared to the data of Poulikakos. Also, no change in the number of circulating cells is seen at Rayleigh numbers greater than $2 \times 10^{5}$. However, the maximum difference in the Nusselt numbers is only about $6 \%$. The present study uses a one-domain formulation, similar to that in the Poulikakos study [20], and the agreement with his results provides sufficient verification for the present numerical scheme.

On the other hand, the numerical results differ significantly with the results of Poulikakos et al. [21] as can be seen from Figure 4.7(b). This can be attributed to the different modeling of the interfacial boundary conditions. Poulikakos et al. [21] use the Beavers-Joseph slip boundary condition at the interface which differs from the continuity boundary conditions implicitly assumed in the present model. As mentioned earlier, no unique formulation for the interfacial boundary conditions exists in the literature and hence it is impossible to truly ascertain the validity of the present model by comparison to prior studies. This issue is revisited in the final chapter while commenting on the comparison between the numerical and experimental results.

\subsection{Convection in fluid-superposed porous layers heated locally}

The dimensionless governing equations for the present problem contain seven dimensionless parameters which determine the overall heat transfer characteristics. These parameters are the heater-to-base length ratio, $\delta$, the porous layer-to-total height ratio, $\eta$, the overall aspect ratio, $A$, the Darcy number, $D a$, the fluid Prandtl number, $\operatorname{Pr}$, the solid-to-fluid conductivity ratio, $\lambda$, and the overall Rayleigh number, $R a$. The presence of so many independent parameters not only makes a complete computational study of the problem a formidable task, it also makes a systematic presentation of the results a challeng-

ing one. In order to perform a thorough parametric analysis of the problem, the effect of each individual parameter on the flow and temperature fields, and the overall heat transfer rates, is studied and analyzed separately. This will aid in identifying the most important parameters and developing a general heat transfer correlation that is applicable to a wide range of problems where this system is encountered. The values of the parameters used in the calculations are summarized in Table 4.2.

\subsubsection{Effect of the heater-to-base length ratio}

The effect the heater length ratio will be considered first. In order to clearly understand the effects of having a localized heat source at the base, it is important to first understand the flow and temperature fields. Figures 4.8, 4.9 and 4.10 show the steady-state 
(a)

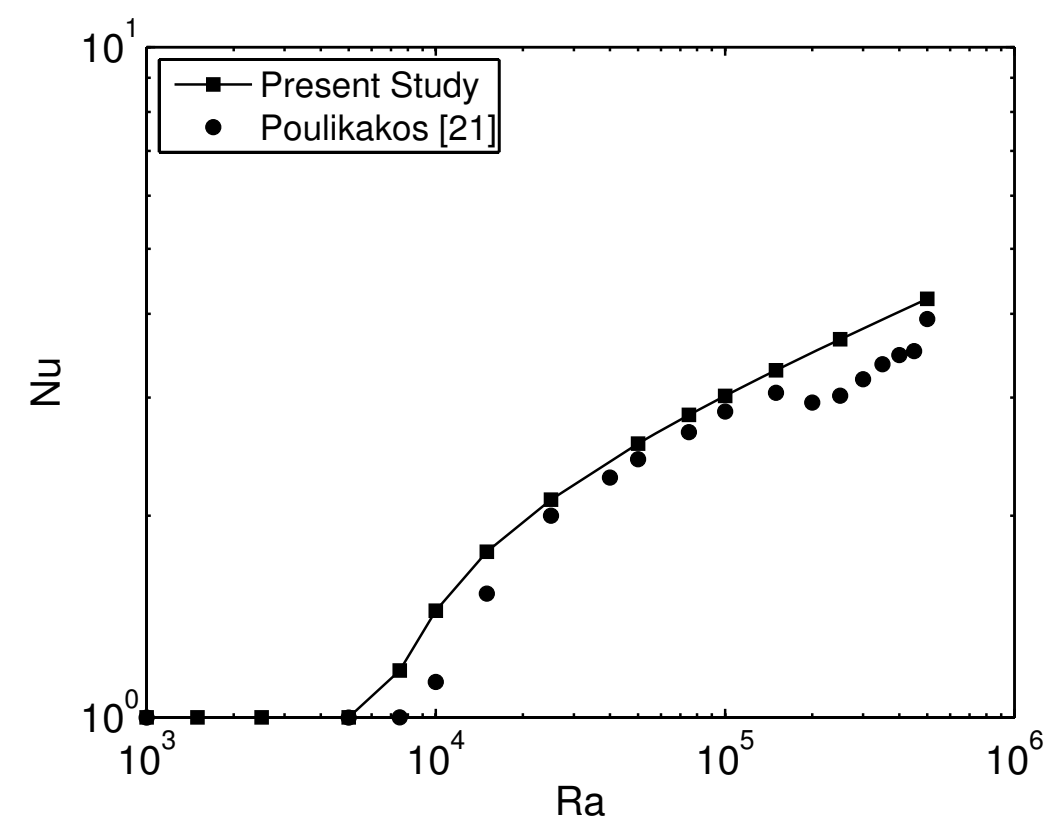

(b)

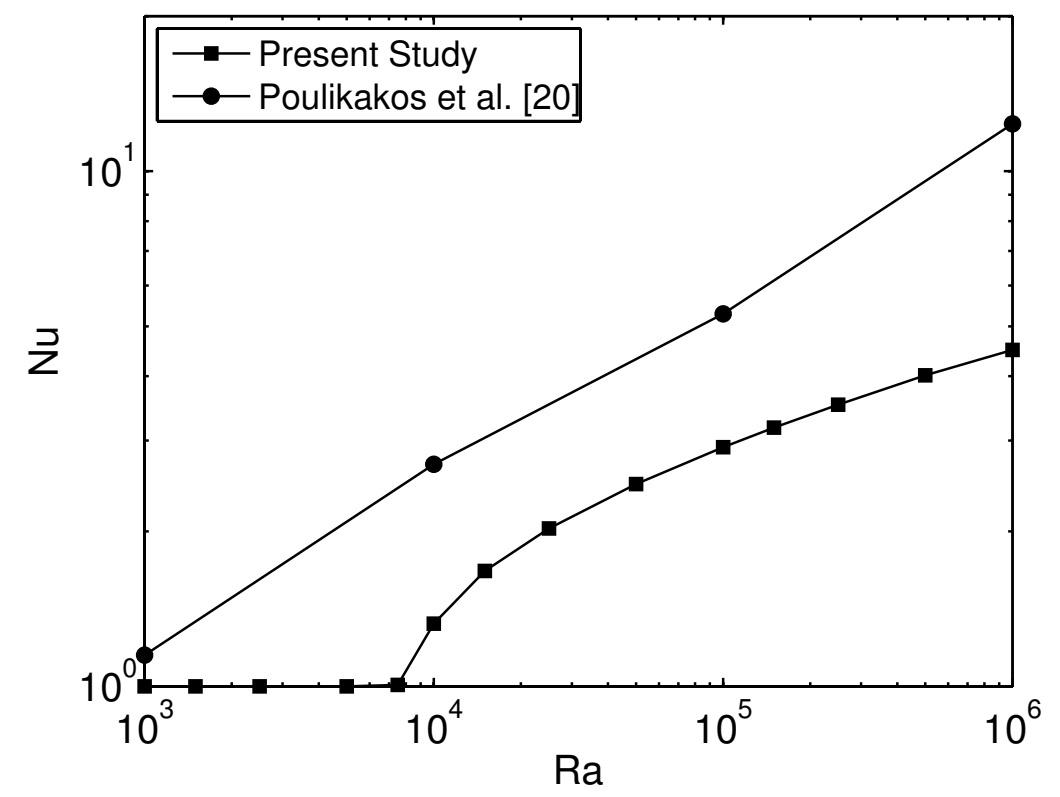

Figure 4.7: Nusselt-versus-Rayleigh number relations for convection in fluid-superposed porous layers. (a) Comparison with numerical results of Poulikakos [21]. (b) Comparison with numerical results of Poulikakos et al. [20]. 
Table 4.2: Values of the parameters used in the various simulations.

\begin{tabular}{|c|c|c|c|c|}
\hline Section & $\begin{array}{l}\text { Parameters } \\
\text { Studied }\end{array}$ & Range of Values & $\begin{array}{l}\text { Fixed } \\
\text { Parameters }\end{array}$ & Grid Size \\
\hline $\begin{array}{l}\text { 4.3.1 Effect of } \\
\text { the heater } \\
\text { length } \\
\text { fraction }(\delta)\end{array}$ & $R a, \delta$ & $\begin{array}{l}R a=10^{3}-10^{6} \\
\delta=0.25,0.5,1\end{array}$ & $\begin{array}{l}\eta=0.5 \\
D a=10^{-6} \\
A=2 \\
\operatorname{Pr}=7 \\
\lambda=1\end{array}$ & $\begin{array}{l}40 \times 80\left(R a<10^{4}\right) \\
52 \times 104\left(R a<10^{6}\right) \\
64 \times 128\left(R a \geq 10^{6}\right)\end{array}$ \\
\hline $\begin{array}{l}\text { 4.3.2 Effect of } \\
\text { the height } \\
\text { ratio }(\eta)\end{array}$ & $R a, \eta$ & $\begin{array}{l}R a=10^{3}-10^{6} \\
\eta=0.25,0.5,0.75\end{array}$ & $\begin{array}{l}\delta=0.5 \\
D a=10^{-6} \\
A=2 \\
\operatorname{Pr}=7 \\
\lambda=1\end{array}$ & $\begin{array}{l}\text { For } \eta=0.25,5 \\
40 \times 80\left(R a<10^{4}\right) \\
52 \times 104\left(R a<10^{6}\right) \\
64 \times 128\left(R a \geq 10^{6}\right) \\
\text { For } \eta=0.75 \\
40 \times 80\left(R a<10^{4}\right) \\
80 \times 160\left(R a>10^{4}\right)\end{array}$ \\
\hline $\begin{array}{l}\text { 4.3.3 Effect of } \\
\text { the Darcy } \\
\text { number }(D a)\end{array}$ & $R a, D a$ & $\begin{array}{l}R a=10^{3}-10^{6} \\
D a= \\
10^{-2}, 10-4,10^{-6}\end{array}$ & $\begin{array}{l}\delta=0.5 \\
\eta=0.5 \\
A=2 \\
\operatorname{Pr}=7 \\
\lambda=1\end{array}$ & $\begin{array}{l}40 \times 80\left(R a<10^{4}\right) \\
52 \times 104\left(R a<10^{6}\right) \\
64 \times 128\left(R a \geq 10^{6}\right)\end{array}$ \\
\hline $\begin{array}{l}\text { 4.3.4 Effect of } \\
\text { the aspect } \\
\text { ratio }(A)\end{array}$ & $R a, A$ & $\begin{array}{l}R a=10^{3}-10^{6} \\
A=2,4,6 \\
\delta=0.5(A=2,4,6) \\
\delta=0.75(A=6)\end{array}$ & $\begin{array}{l}\eta=0.5 \\
D a=10^{-6} \\
\operatorname{Pr}=7 \\
\lambda=1\end{array}$ & $\begin{array}{l}\text { For } A=2 \\
40 \times 80\left(R a<10^{4}\right) \\
52 \times 104\left(R a<10^{6}\right) \\
64 \times 128\left(R a \geq 10^{6}\right) \\
\text { For } A=4,6 \\
40 \times 80\left(R a<10^{4}\right) \\
64 \times 128\left(R a>10^{4}\right)\end{array}$ \\
\hline $\begin{array}{l}\text { 4.3.5 Effect of the } \\
\text { conductivity } \\
\text { ratio }(\lambda)\end{array}$ & $R a, \lambda$ & $\begin{array}{l}R a=10^{3}-10^{6} \\
\lambda=1,50,100\end{array}$ & $\begin{array}{l}\delta=0.5 \\
\eta=0.5 \\
D a=10^{-6} \\
A=2 \\
\operatorname{Pr}=7\end{array}$ & $\begin{array}{l}40 \times 80\left(R a<10^{4}\right) \\
52 \times 104\left(R a<10^{6}\right) \\
64 \times 128\left(R a \geq 10^{6}\right)\end{array}$ \\
\hline $\begin{array}{l}\text { 4.3.6 Effect of } \\
\text { the Prandtl } \\
\text { number }(P r)\end{array}$ & $R a, \operatorname{Pr}$ & $\begin{array}{l}R a=10^{3}-10^{6} \\
\operatorname{Pr}=0.7,7\end{array}$ & $\begin{array}{l}\delta=0.5 \\
\eta=0.5 \\
D a=10^{-6} \\
A=2 \\
\lambda=1\end{array}$ & $\begin{array}{l}40 \times 80\left(R a<10^{4}\right) \\
52 \times 104\left(R a<10^{6}\right) \\
64 \times 128\left(R a \geq 10^{6}\right)\end{array}$ \\
\hline
\end{tabular}


(a)

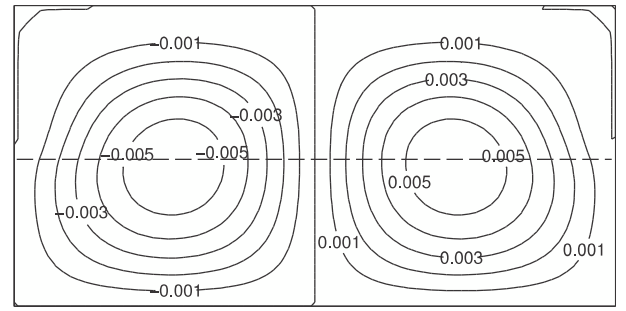

(b)

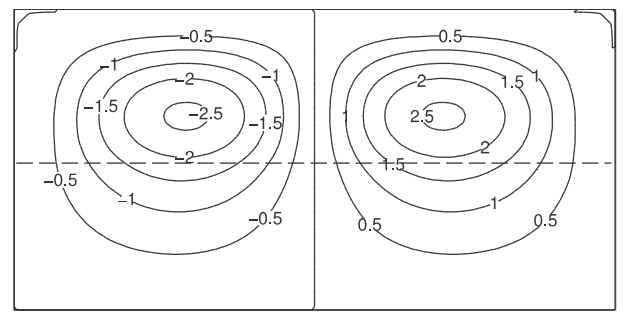

(c)

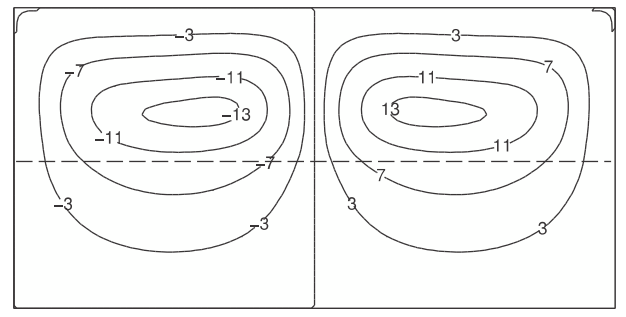

(d)

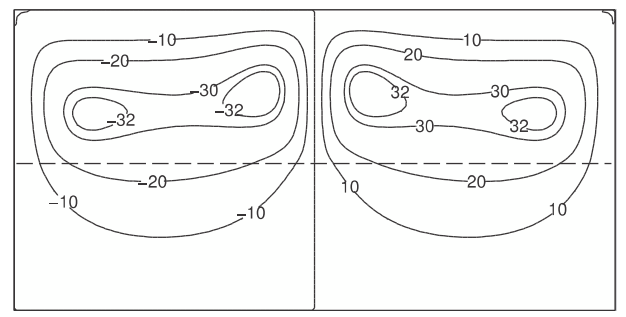

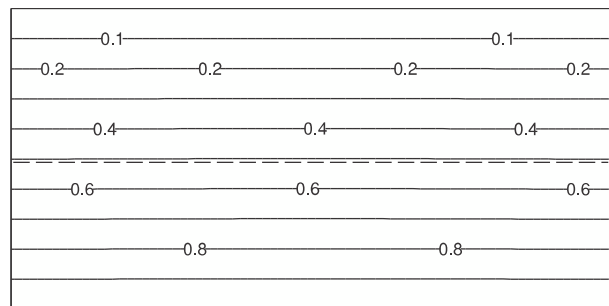
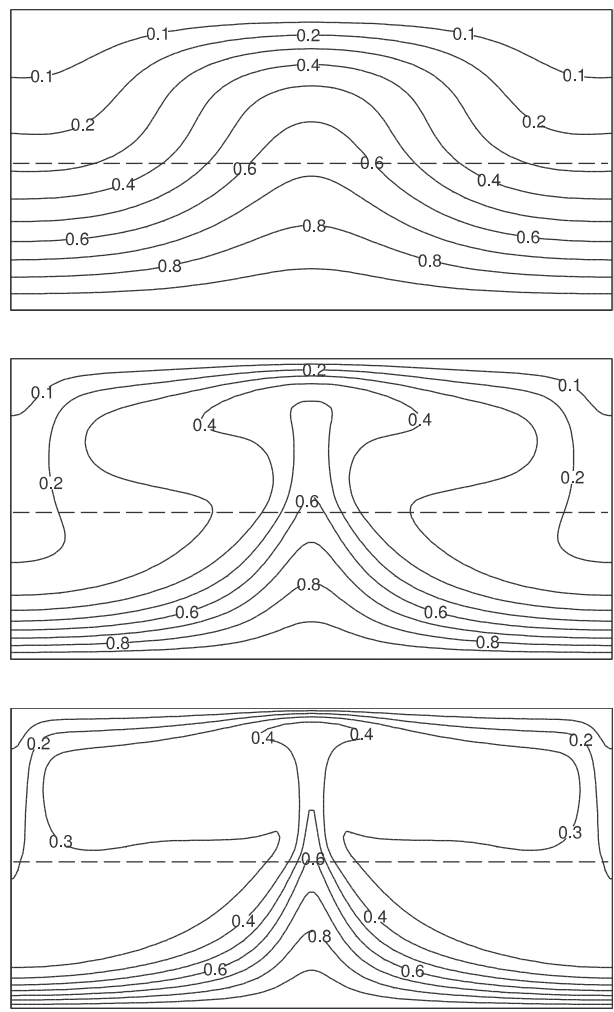

Figure 4.8: Streamline and isotherm patterns for convection in a composite layer for $\delta=1$ and $\eta=0.5$. (a) $R a=10^{3}$, (b) $R a=10^{4}$, (c) $R a=10^{5}$, and (d) $R a=10^{6}$.

streamline and isotherm patterns for $\eta=0.5$ and $\delta=1,0.5$ and 0.25 , respectively at four different Rayleigh numbers. For $\delta=1$, i.e., when the base is uniformly heated, there is no convective motion at $R a=10^{3}$ and the system is in the conduction mode. Although the streamline patters show a cellular convective motion, these are meaningless as the gradient of the stream function is extremely low. When the Rayleigh number increases to $10^{4}$, however, convective motion commences and two pairs of convective cells circulating in opposite directions appear. The corresponding isotherms show a rising thermal plume.

It may be noted that convective motion is confined to the overlying fluid layer with some penetrative convection in to the underlying porous layer. The issue of penetrative convection in composite systems has been the subject of a large number of numerical studies in the literature, but no definitive conclusion can be drawn from the evidence available. Some 
(a)
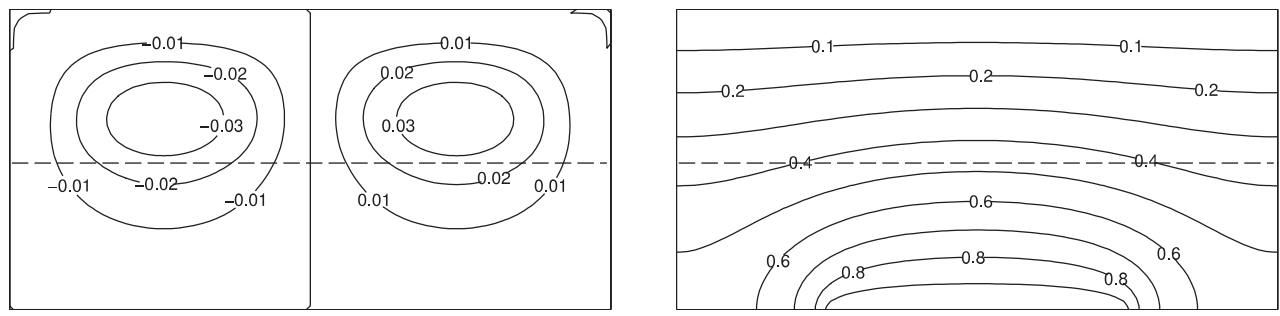

(b)
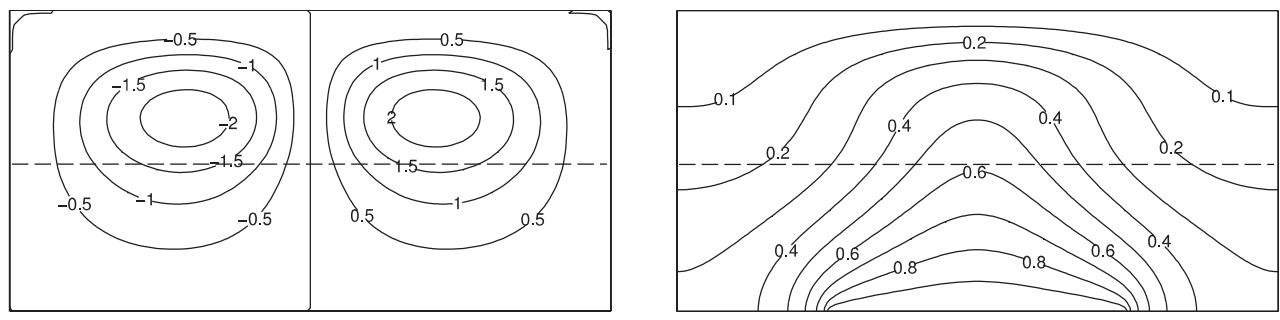

(c)
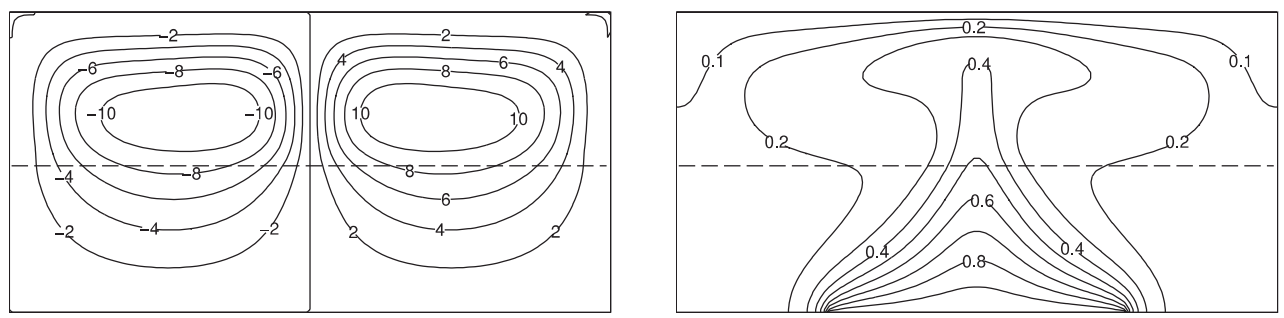

(d)
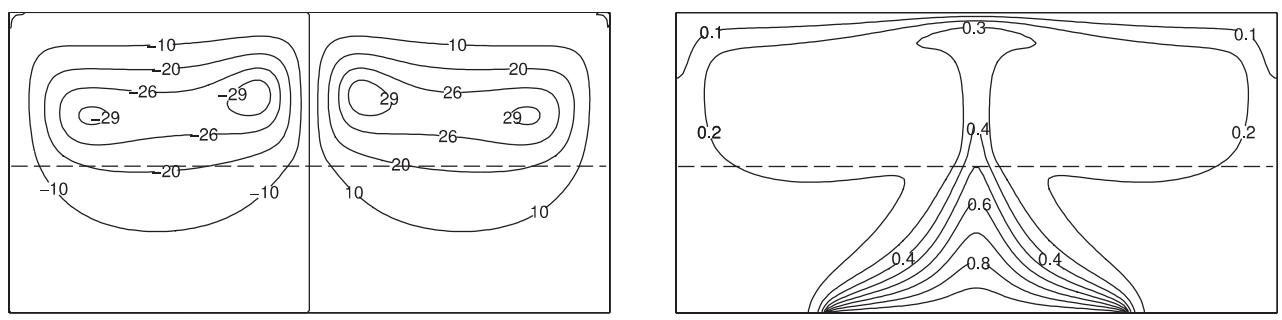

Figure 4.9: Streamline and isotherm patterns for convection in a composite layer for $\delta=0.5$ and $\eta=0.5$. (a) $R a=10^{3}$, (b) $R a=10^{4}$, (c) $R a=10^{5}$, and (d) $R a=10^{6}$.

studies report the occurrence of penetrative convection $[20,21]$ while others $[22,23]$ report that convection is limited to only the upper fluid layer with minimal or no penetration in to the lower porous layer. This lack of argument cannot be simply attributed to the modeling of the interfacial boundary conditions given that studies using a particular boundary condition have reported different results. Visualization studies by Prasad and co-workers have shown that penetrative convection does indeed occur [24-26]. However, owing to the highly porous nature and large pore sizes of the porous media used in their studies, this conclusion cannot be considered to be generic. At this point, it is sufficient to say that the present results are in accordance with the mathematical formulation of the problem which assumes the continuity of $x$ - and $y$-direction velocities across the porous-fluid layer interface, and hence, implicitly allows for penetrative convection. Thus the present results indicate that 
(a)

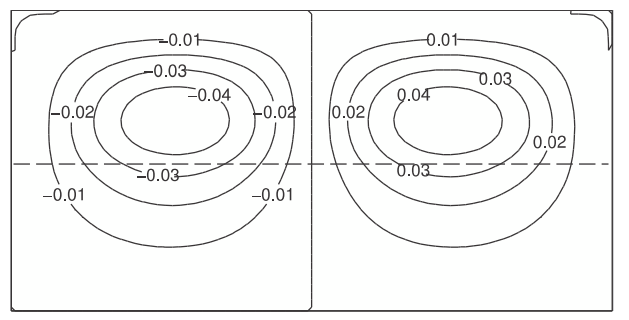

(b)

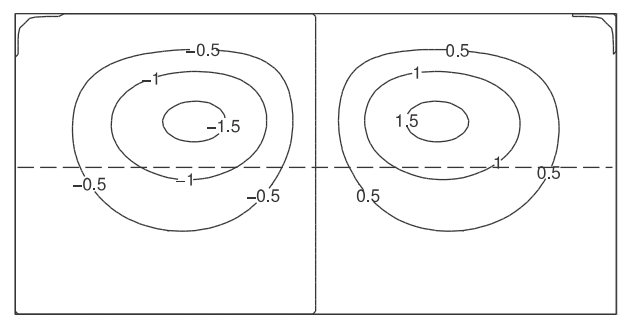

(c)

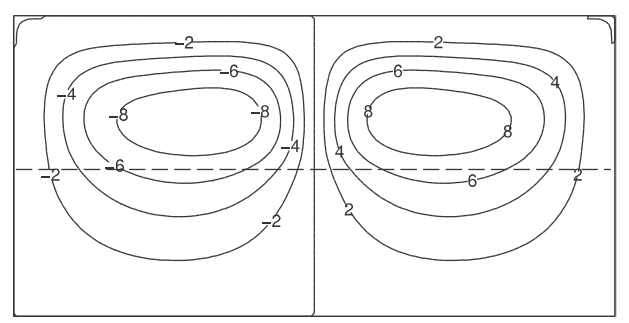

(d)

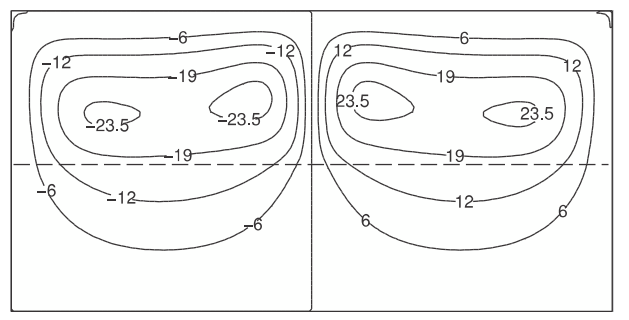

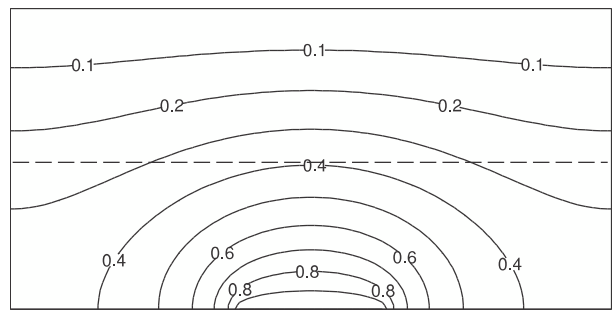
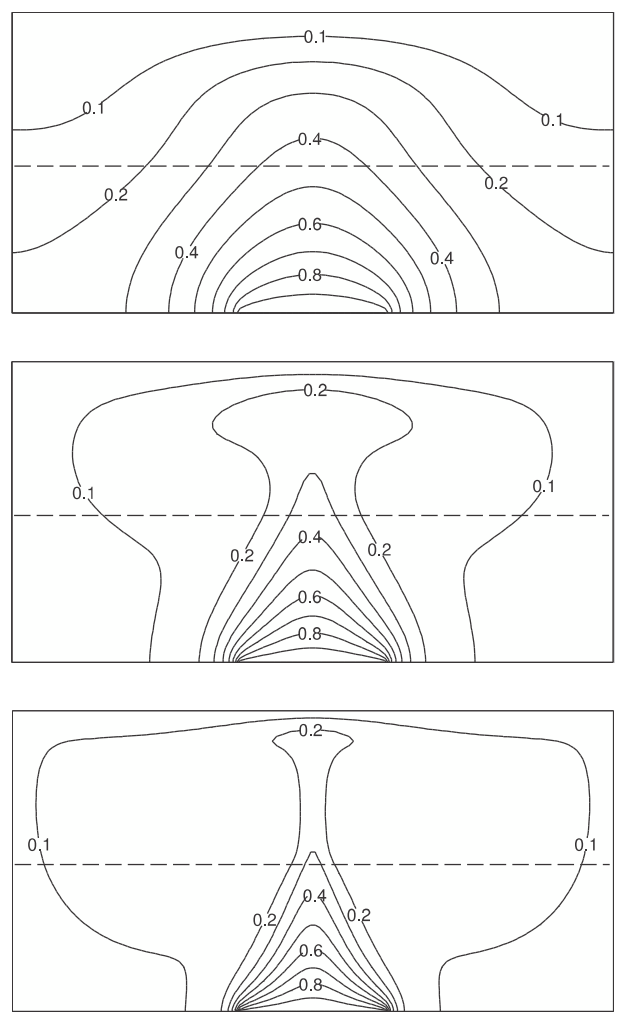

Figure 4.10: Streamline and isotherm patterns for convection in a composite layer for $\delta=$ 0.25 and $\eta=0.5$. (a) $R a=10^{3}$, (b) $R a=10^{4}$, (c) $R a=10^{5}$, and (d) $R a=10^{6}$.

beyond the critical Rayleigh number, a state of pure conduction with no fluid motion in the underlying porous layer never exists. It must be noted, however, that convective motion in the porous layer occurs primarily in the vicinity porous-fluid layer interface. In the region away from the interface, there is little convective motion and heat transfer is mainly by conduction.

With increase in the Rayleigh number to $10^{5}$, the velocities of the convective motion in the overlying fluid layer increase, though the extent of flow penetration in to the lower porous layer does not seem to increase significantly. The individual cells appear to be horizontally stretched and the isotherm patterns show a narrow plume rising along the centerline of the cavity. With further increase in the Rayleigh number to $10^{6}$, this plume becomes narrower and the convective motion in the upper fluid layer becomes more intense. 
When the size of the heater covers only half of the base, i.e., for $\delta=0.5$, the isotherm patterns change significantly. However, the streamline patters show little noticeable change except that at $R a=10^{3}$ a cellular convective pattern can be seen. Though the gradient of the stream function at this Rayleigh number is small, it is not negligible, which indicates that a circulatory fluid motion with low velocity exists. This fluid motion does not however significantly enhance the overall heat transfer rate and the isotherms are essentially identical to ones obtained for the conduction solution $(R a \sim 1)$. This shows that convection is not the dominant mode of heat transfer and, hence, the cellular fluid motion cannot be attributed to the onset of convective flow. Rather, the circulatory fluid motion arises due to the presence of the localized heat source. At the edge of the heated section of the base a horizontal temperature gradient exists which triggers a circulatory fluid motion. This phenomenon has also been observed in studies on convection in porous and fluid layers with localized heat sources $[20-22,27]$. These cells are often referred to as "end cells" as they primarily appear at the heater edges.

An interesting observation that can be made is that these cells are not located at the heater edges, but rather in the overlying fluid layer. This phenomenon can be explained by recalling that for convection in fluid-superposed porous layers, fluid motion is always confined to the overlying fluid layers except for very large height ratios $(\eta>0.91)$. The present results show that this is true even when the heat source is localized. Thus, irrespective of whether the source of instability is either latent in the system or imposed by a local heat source, the mode of convection is essentially the same. This is a significant finding of the present study and shows that localized heat sources do not change the fundamental convective modes. Rather the heat source merely acts as a trigger for the onset of convective motion and the ensuing flow patterns remain unaffected by whether heating at the base is uniform or localized.

With increasing $R a$, the overall nature of the flow remains essentially the same as that for a uniform heat source. The flow remains confined to the fluid layer and the extent of penetration in to the lower porous layer does not increase. The isotherms however differ significantly from those of a uniform heat source. With increasing $R a$, the thermal plume rising above the heat source is seen to become narrower, as seen in the case of a uniformly heated source. However, the porous layer outside the pluming region and away from the interface remains essentially isothermal and does not participate in heat transfer. The temperature in this quiescent region is very close to the upper surface temperature, indicating that region essentially has a pool of cold stagnant fluid. This situation is in contrast to the case of a uniformly heated base where there porous region away from the plume has heat transfer by conduction.

A further decrease in the heater length ratio to $\delta=0.25$ shows much of the same 


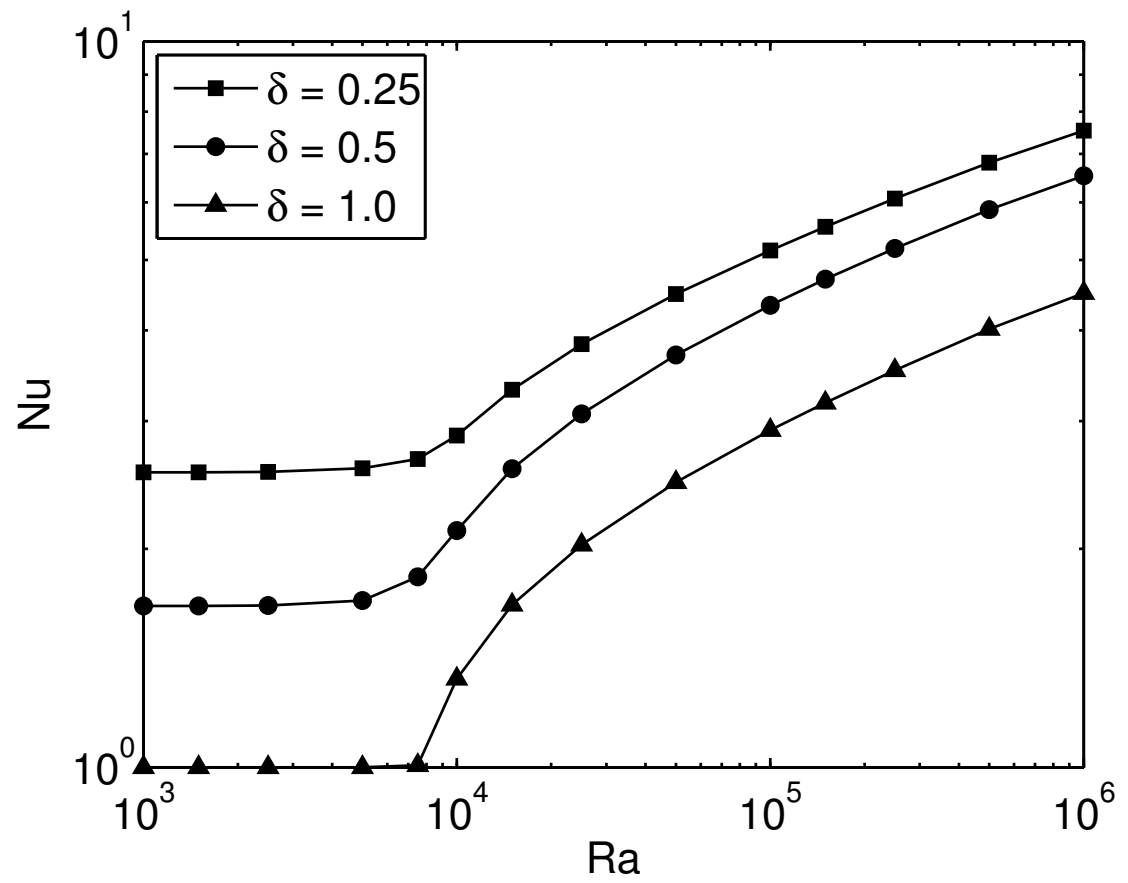

Figure 4.11: Nusselt-versus-Rayleigh number relationship for different heater length ratios and $\eta=0.5$.

characteristics as described above. A circulatory pattern is seen to exist at $R a=10^{3}$ and its form is almost the same as that seen for $\delta=0.5$, further confirming that is pattern is merely due to the instability created by a horizontal temperature gradient at the heater edge. At higher Rayleigh numbers, the streamline patterns are similar to those for $\delta=$ 0.5 which shows that the flow patterns are unaffected by the heater size. The isotherm patterns show a narrow plume rising from the center of the heater and, as noted earlier, the porous region outside the pluming zone remains relatively unaffected and is essentially isothermal. Interestingly, much of the fluid region, and hence, much of the composite domain is essentially isothermal at $R a=10^{6}$. This is likely to get more pronounced as the heater size shrinks further until, in the limiting case of a point heat source, the region outside the rising plume is likely to be completely quiescent.

The effect of the localized heat source on the overall heat transfer coefficient is shown in Figure 4.11. For a uniformly heated base, the critical Rayleigh number for the onset of convection is $\sim 7.5 \times 10^{3}$ below which the average Nusselt number is unity indicating that heat transfer occurs solely by conduction. When the heater size is finite, however, the Nusselt number is no longer unity even at low values of $R a$. This is simply a consequence of the fact that the presence of a localized heat source initiates a circulatory motion due to the temperature gradient at the heater edge, as mentioned above. Hence a pure conduction state never exists for a localized heat source. This, however, does not imply the dominant mode of 
heat transfer is convection. As Figure 4.11 shows, even for discrete heat sources, the Nusselt number remains approximately constant till the critical point is reached. Hence while the pre-critical point heat transfer regime is not purely a conduction regime, it certainly is conduction dominated. Also noteworthy is that while the transition to the convective regime is a discrete one for $\delta=1$, the transition to the convective regime is a smooth one. This aspect has been previously observed for convection in porous and fluid layers with discrete heat sources $[29,57-61]$ and is a consequence of the fact that when the heat source is localized, a circulatory pattern already exists in the cavity before convection becomes dominant. As such, there is no sudden initiation of convective motion but a gradual strengthening of the existing base flow pattern.

The most interesting aspect however is that over the entire range of Rayleigh number, the average Nusselt number over the heater increases with decrease in the size of the heat source. This too has been previously observed with respect to convection in pure fluid and porous layers. This observation can be understood by going back to the discussion on the flow and isotherm patterns. As mentioned earlier, for a uniformly heated base, heat transfer away from the pluming region is primarily by conduction, which for a low solidto-fluid conductivity ratio ( $\lambda=1$ here) is fairly low. On the other hand, for a discrete heater, most the heater area falls in the pluming region and hence almost all the heat input is carried away by convection. In this case conduction heat transfer occurs only near the edges of the heated section. With further decrease in $\delta$ almost all the energy supplied is carried away by convective flow. Therefore with a decrease in the size of the heater more and more of the energy input at the base is transferred to the top via convection. It can thus be deduced that a localized heat source is more efficient in channeling away the energy influx at the heater via the rising thermal plume thus leading to higher Nusselt numbers for lower heater lengths.

Finally, to conclude the discussion on the effects of a localized heat source, the temperature profiles along the centerline of the cavity at $R a=10^{5}$, are shown in Figure 4.12. It can be seen that with decrease in the size of the heated fraction of the base, the temperature profiles along the centerline of the plume change significantly. For example the temperature at the interface $(y / H=0.5)$ decreases with decrease in the heater length. This is true for temperatures throughout the entire cavity height. This can be understood by looking at the isotherm patterns. With decreasing heater size, much of the temperature drop along the plume occurs very close to the heater while in the rest of the plume, the temperature changes gradually. This directly leads to the fact that with decreasing heater size, the plume draws away more and more of the heat that is supplied to the system. 


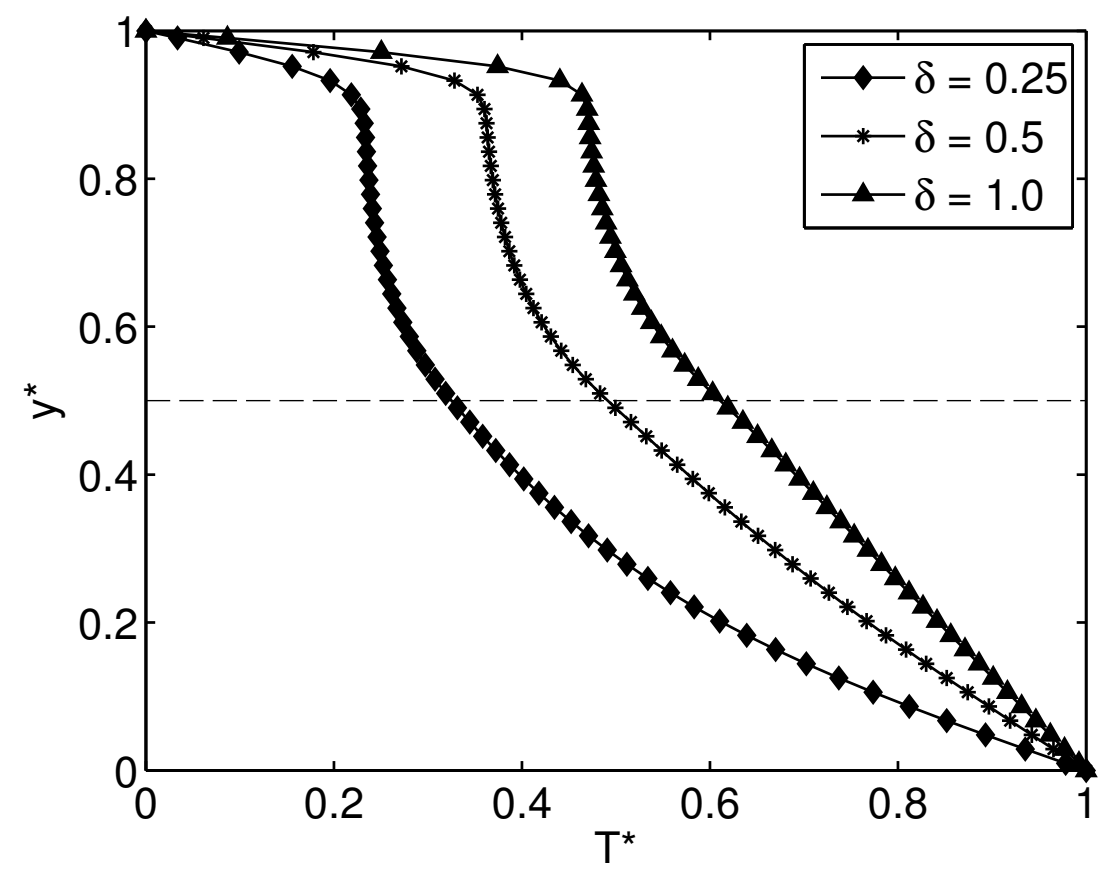

Figure 4.12: Dimensionless temperature $\left(T^{*}=T-T_{C} / T_{H}-T_{C}\right)$ profiles along the vertical centerline of the domain for different heater length ratios. $\eta=0.5$ and $R a=$ $5 \times 10^{5}$.

\subsubsection{Effect of the porous layer-to-total height ratio}

Similar to the approach adopted earlier, description of the effect of the porous-tototal height ratio will begin with a discussion of the flow and temperature fields at different height ratios. In these simulations, all parameters are kept fixed except for the height ratio. Figures 4.13, 4.14 and 4.15 show the streamline and isotherm patterns for three different height ratios, $\eta=0.5,0.25$ and 0.75 , respectively, and $\delta=0.5, A=2, D a=10^{-6}, \operatorname{Pr}=7$, and $\lambda=1$.

First, the case of $\eta=0.5$ will be discussed as this case $(\eta=0.5, \delta=0.5)$ has already been encountered in the previous section. To briefly summarize the previous discussion, the salient feature of the flow pattern is that the convective motion is restricted to the overlying fluid layer with some penetration in to the underlying porous layer. Also, a circulatory motion triggered by the presence of a localized heat source is seen at $R a \leq 10^{3}$, although convection is still not the dominant mode of heat transfer. These two observations point to the fact that a state of pure conduction heat transfer never exists in the underlying porous layer when a localized heat source is present. Thus a sharp critical point for the onset of convection cannot be defined for a localized heat source; a critical point in this case merely indicates that convection becomes the dominant mode of heat transfer. With increase in the Rayleigh number, the velocities of convective motion increase and at very high $R a$ 
(a)
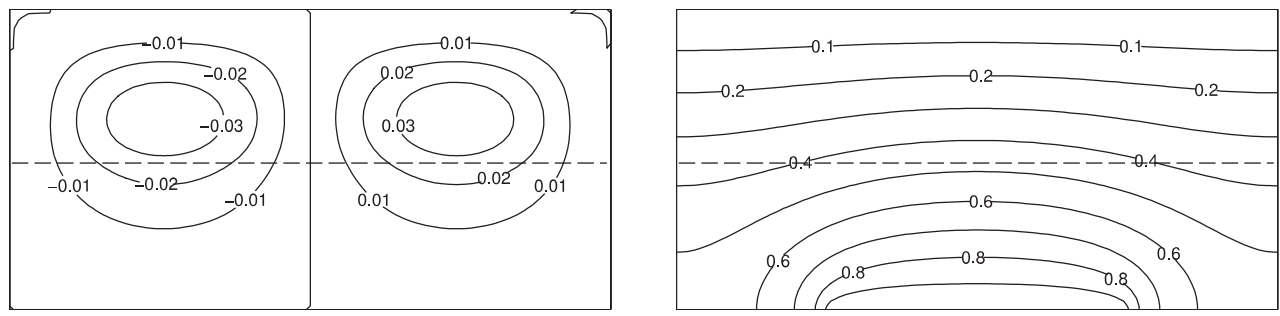

(b)
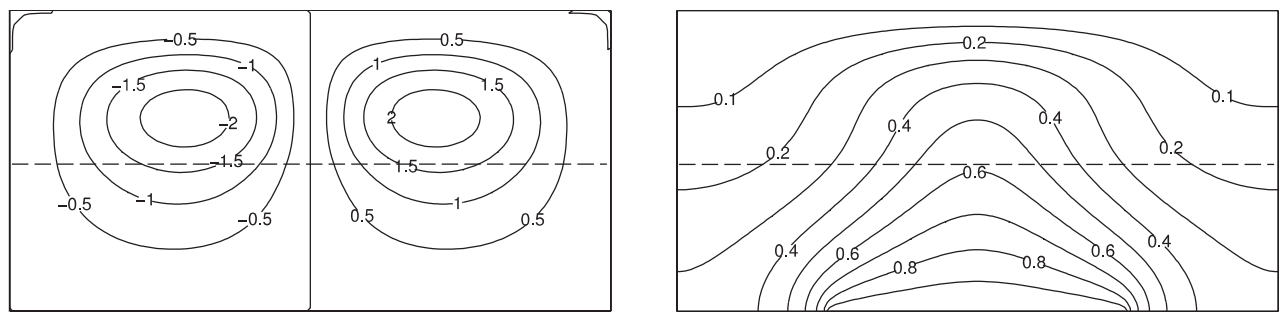

(c)
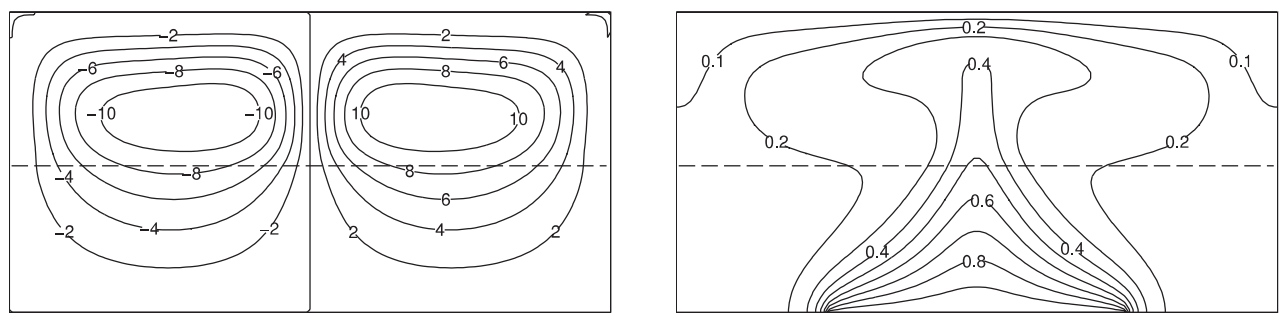

(d)
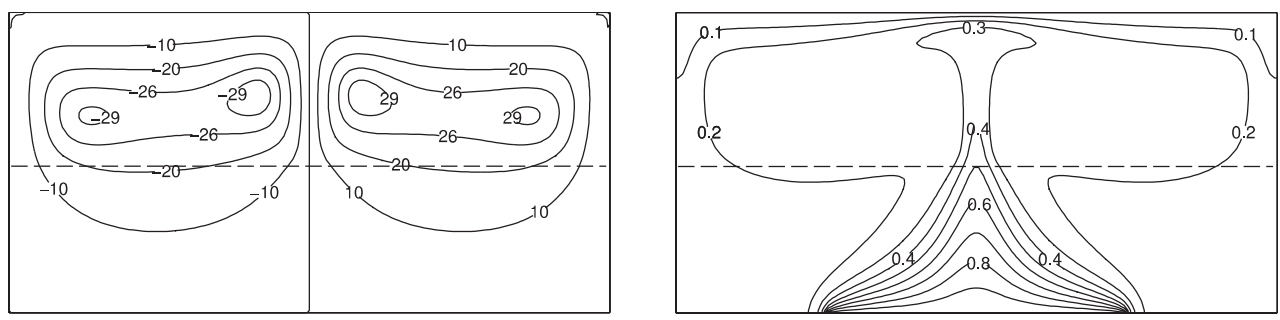

Figure 4.13: Streamline and isotherm patterns for convection in a composite layer for $\delta=$ 0.5 and $\eta=0.5$. (a) $R a=10^{3}$, (b) $R a=10^{4}$, (c) $R a=10^{5}$, and (d) $R a=10^{6}$.

recirculating patterns can be seen within the same convective cell. The isotherm patterns for $\eta=0.5$ indicate that that with increasing $R a$, the plume rising from the central portion of the heater becomes narrower and the region outside the plume in the porous layer is essentially isothermal.

When the height ratio drops to $\eta=0.25$, i.e., when only a quarter of the entire cavity is occupied by the porous layer, the streamline and isotherm patterns are very much similar to those obtained for pure fluid convection with a localized heat source. As noted earlier, the convection motion is restricted to the overlying fluid layer with some penetration in to the underlying porous layer. However, since three-quarters of the entire domain is occupied by the fluid layer, convective motion exists in almost the entire cavity. At $R a=10^{3}$, circulatory motion driven by the localized heat source can be seen. The absolute maximum 
(a)

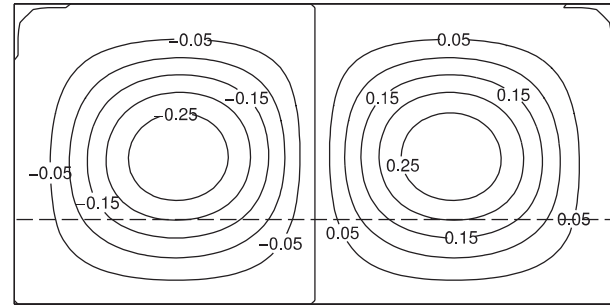

(b)

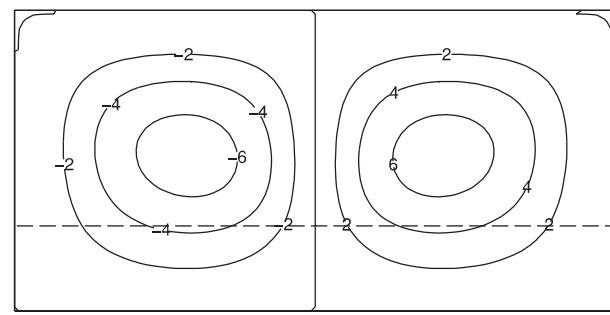

(c)

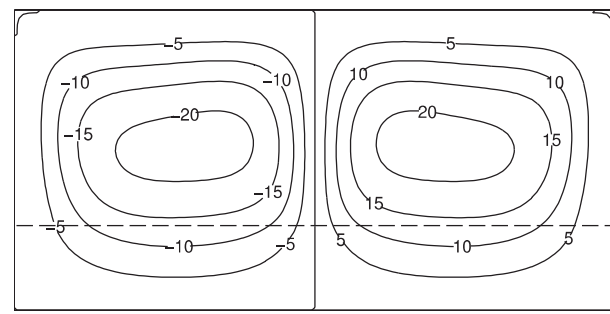

(d)

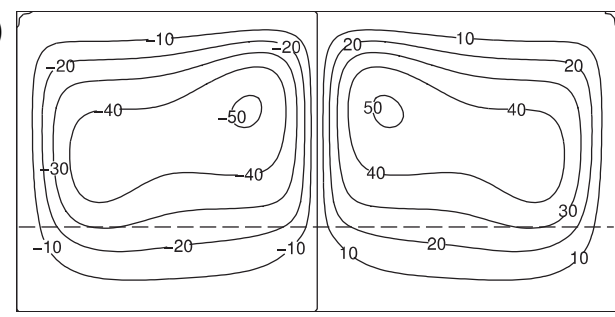

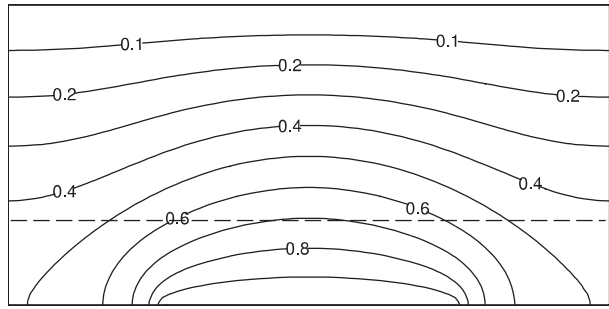
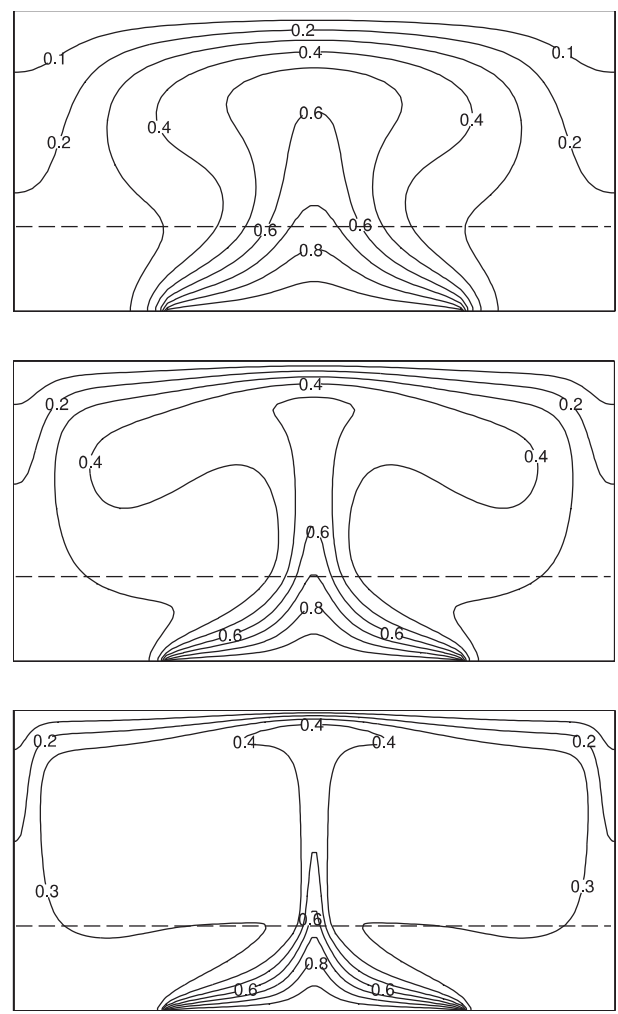

Figure 4.14: Streamline and isotherm patterns for convection in a composite layer for $\delta=$ 0.5 and $\eta=0.25$. (a) $R a=10^{3}$, (b) $R a=10^{4}$, (c) $R a=10^{5}$, and (d) $R a=10^{6}$.

value of the stream function at this Rayleigh number, however, is an order of magnitude higher than that for $\eta=0.5$ indicating that the intensity of fluid motion is much higher. This flow behavior is to be expected generally considering the fact that the porous layer provides the greatest resistance to fluid motion within the composite domain. Therefore a reduction in the total amount of porous matrix in the domain reduces the overall resistance to fluid motion and leads to higher fluid velocities. This trend is noticed at all values of $R a$. Based on this observation, it can be deduced that the overall heat transfer rate increases with a decrease in the height ratio owing to a reduction in the resistance to fluid motion by the porous layer and an associated increase in convection. With an increase in the Rayleigh number the velocity of the fluid motion increases. The shape of the convection cells however is different from those of $\eta=0.5$. The cells are almost square in shape and 
(a)

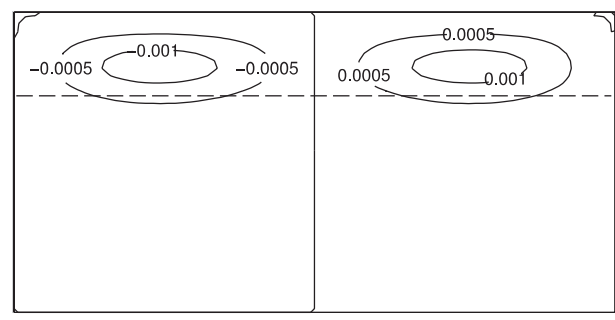

(b)

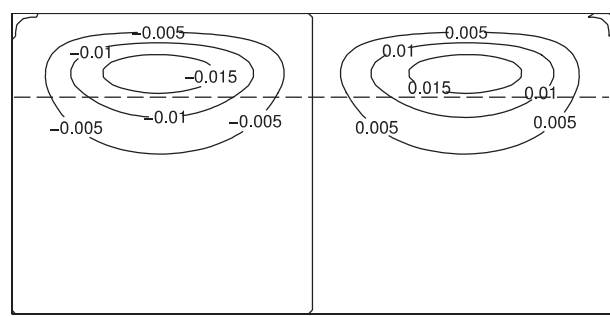

(c)

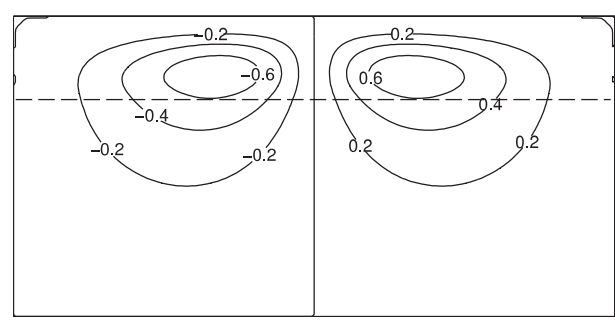

(d)

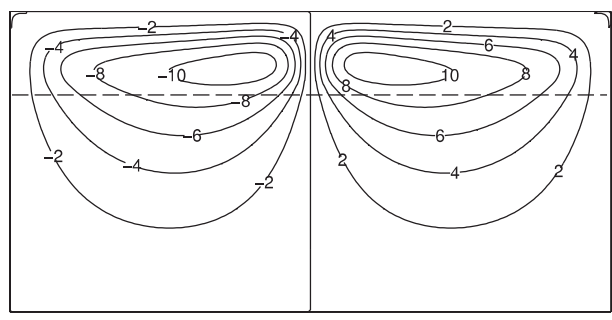

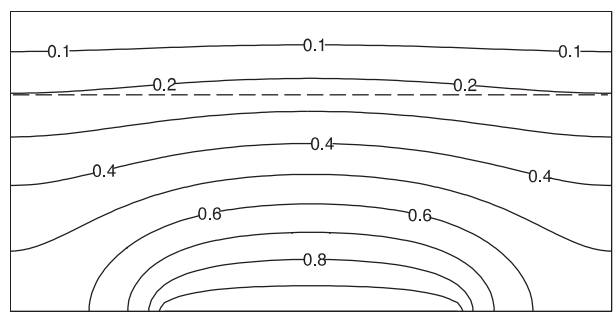
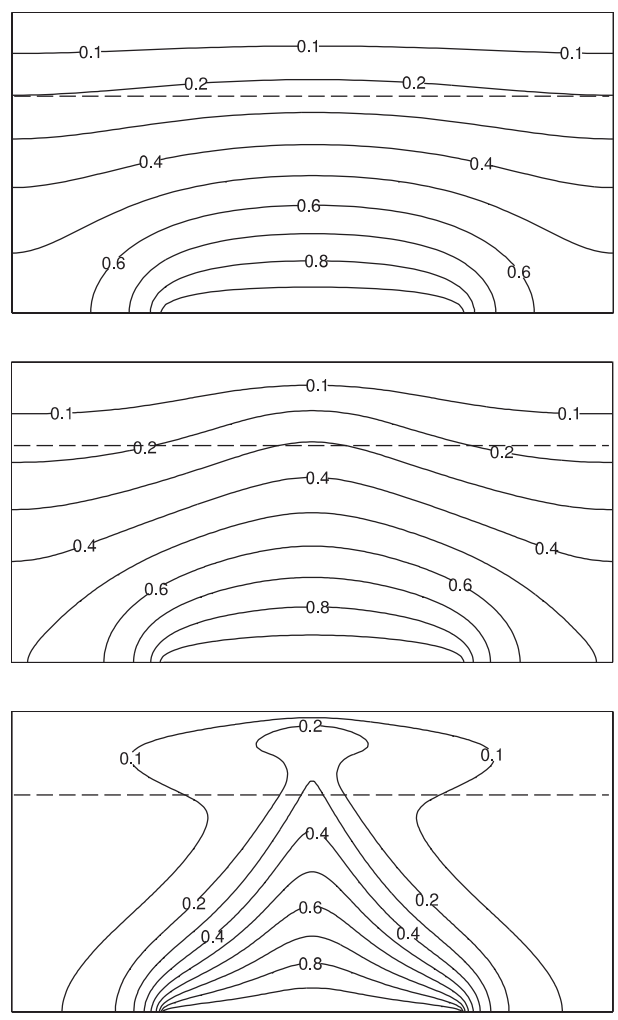

Figure 4.15: Streamline and isotherm patterns for convection in a composite layer for $\delta=$ 0.5 and $\eta=0.75$. (a) $R a=10^{3}$, (b) $R a=10^{4}$, (c) $R a=10^{5}$, and (d) $R a=10^{6}$.

the size of the core region, where the flow velocities are highest, is noticeably larger as a direct consequence of the convection being dominated by the overlying fluid layer. As the fluid motion is restricted primarily to the fluid layer, the size and shape of the circulating cells adjust to fit the fluid space. With the aspect ratio fixed, the width of the cells are constrained. However the height of the cells changes with change in the height of the fluid layer. Thus, as the fluid layer height increases, the cells become squarer; the opposite happens as the fluid height layer decreases. At $R a=10^{6}$, two pockets of recirculating cells can be seen on opposite sides of the same convective cell and their size is noticeable larger.

The isotherm patterns are similar to those observed for $\eta=0.5$. The central feature is a plume like flow along the mid-section of the cavity, which becomes narrower as the Rayleigh number increases. With convective motion being prevalent almost throughout the 
entire cavity, no noticeable quiescent isothermal region exists, even in the porous layer. The plume itself becomes wider at it approaches the upper surface and has an umbrella like shape near the upper surface.

When the porous layer occupies three-quarters of the cavity, i.e., for $\eta=0.75$, the flow and isotherm patterns change significantly. At $R a=10^{3}$, the heater driven instability causes a pair of circulation cells to form. However the maximum absolute value of the stream function is extremely low making the flow velocities almost negligible. Hence it can be concluded that there is little or no fluid motion in the system at this Rayleigh number. When the Rayleigh number increases to $10^{4}$, the circulation velocities are still very low. The isotherms show practically no change indicating that heat transfer is still primarily by conduction. With further increase in $R a$ to $10^{5}$, the isotherm patterns change slightly indicating that convection is taking over as the dominant mechanism of heat transfer. As mentioned earlier, the transition to convection dominated heat transfer takes place gradually for discrete heat sources, and gradual transition can be seen here too. Finally when the Rayleigh number increases to $10^{6}$, a convection dominant flow can be seen with the accompanying plume like flow.

For high values of the height ratio, several important aspects can be noticed. The first is that even at $R a=10^{6}$, convective flow occurs with fairly low velocities as can be seen from the value of the stream function. This is obviously due the fact that increase in the porous layer height increases the resistance to convective motion. Another way to look at it is in terms of the porous medium Rayleigh number, $R a_{\mathrm{m}}$ which is defined as,

$$
R a=\frac{R a_{\mathrm{m}}}{D a \eta^{3} \kappa T_{I}}
$$

where $T_{I}$ is the interface temperature. It has been shown using linear stability theory that with increase in the height of the porous layer, the critical value of $R a_{\mathrm{m}}$ for the onset of convection increases $[7,9]$. From equation (4.1) it can be seen that this increases the value of $R a$ for the onset of convective motion. This implies that with an increase in the height of the porous layer, much higher values of $R a$ are needed to induce convective motion of higher intensities. Another important aspect of the flow is that with an increase in the Rayleigh number, flow penetration into the underlying porous layer increases. This is in contrast to the flow patterns at lower values of $\eta$ where the degree of flow penetration is independent of the Rayleigh number. The reason for this is not entirely clear. It may be that penetration takes place simply to accommodate the flow pattern which does not have sufficient space within the fluid layer to develop. The isotherm patterns indicate that the plume like flow has not fully developed even at $R a=10^{6}$. Based on the previous results, it can be anticipated that with an increase in the Rayleigh number, a narrow plume like flow 


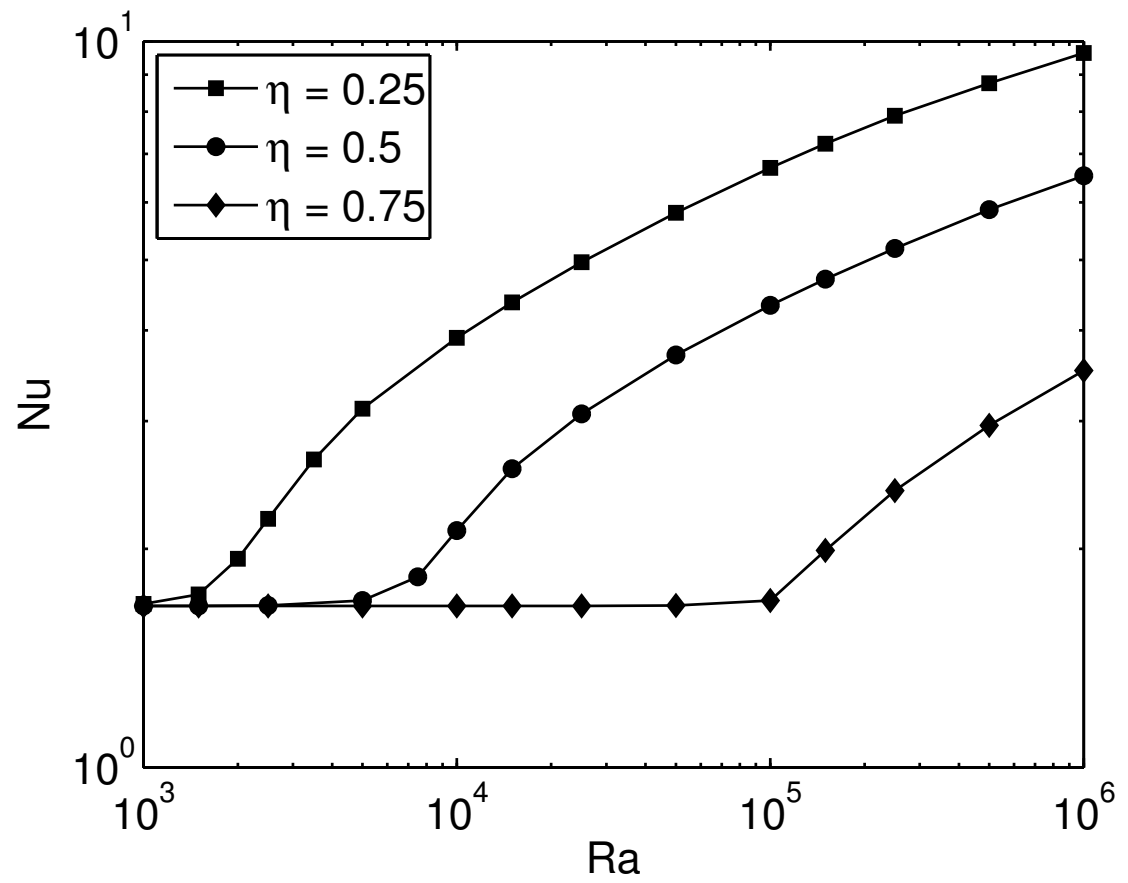

Figure 4.16: Nusselt-versus-Rayleigh number relationship for different height ratios and $\delta$ $=0.5$.

will eventually develop.

Further insights into the effects of the flow fields can be gained by looking at the overall heat transfer coefficients. Figure 4.16 shows the Nusselt-versus-Rayleigh number relation for three different height ratios. The results mirror the inferences that can be drawn from an analysis of the flow and temperature fields. As seen earlier, the onset point for convection dominated heat transfer increases with the increase in the fraction of the cavity occupied by the porous layer, which is also in accordance with the predictions of the linear stability theory. However the Nusselt number in the conduction regime remains the same at all height ratios and is expected as the heater size remains fixed. At Rayleigh numbers higher than the critical point, overall heat transfer coefficients are higher for lower height ratios. This too is a direct consequence of the fact that the velocities of convective motion for a given Rayleigh number is higher when the value of $\eta$ is lower, i.e., when the thickness of the overlying fluid layer is higher. This trend has also been noticed in numerical studies by Chen and Chen [22] and experimental studies by Prasad and co-workers [24-26]. Finally, it must be noted that the Nusselt number curves all have the same pattern irrespective of the height ratio. In fact, a close look at the curves will show that they are almost parallel indicating a common mechanism of heat transfer with a different point of origin that is dictated by the height ratio. This can be further confirmed by looking at the dimensionless temperature profiles along the centerline of the cavity as shown in Figure 4.17. It can be 


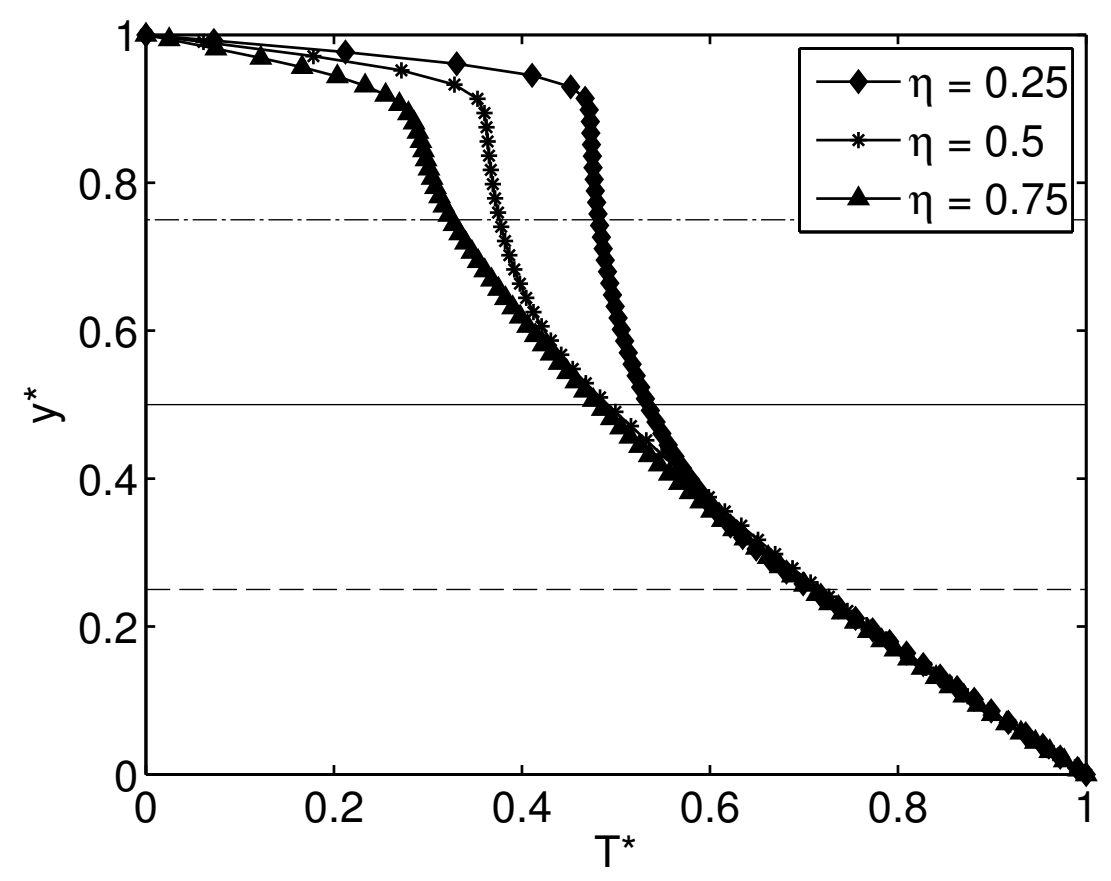

Figure 4.17: Dimensionless temperature $\left(T^{*}=T-T_{C} / T_{H}-T_{C}\right)$ profiles along the vertical centerline of the domain for different height ratios. $\delta=0.5$ and $R a=5 \times 10^{5}$.

seen that the temperature profiles for all height ratios converge to the same value within the porous layer and the difference among them is noticeable only in the fluid layer. Within the fluid layer, centerline temperatures increase with the height ratio leading to higher heat transfer flux at the upper boundary.

\subsubsection{Effect of the Darcy number}

The Darcy number is dimensionless measure of the permeability of the porous medium and it is of vital importance to understand how heat transfer coefficients change with change in the permeability of the system. It must be noted that the Darcy number in the present study is defined as $D a=K / H^{2}$, where $H$ is the overall height of the composite system. This definition of the Darcy number thus depends on the system under consideration and does not express the intrinsic Darcy number of the porous layer which would be based on a length scale suitable for the porous medium. However a Darcy number based on a different length scale can be derived directly from the current definition of $D a$. For example, a Darcy number based on the pore diameter, $d$, can be defined,

$$
D a_{p}=\frac{K}{d^{2}}=D a \gamma^{2}
$$

where $D a_{p}$ is the Darcy number based on the particle diameter, and $\gamma$ is the non-dimensional particle diameter. Thus conclusions based on the current definition of the Darcy number 
(a)
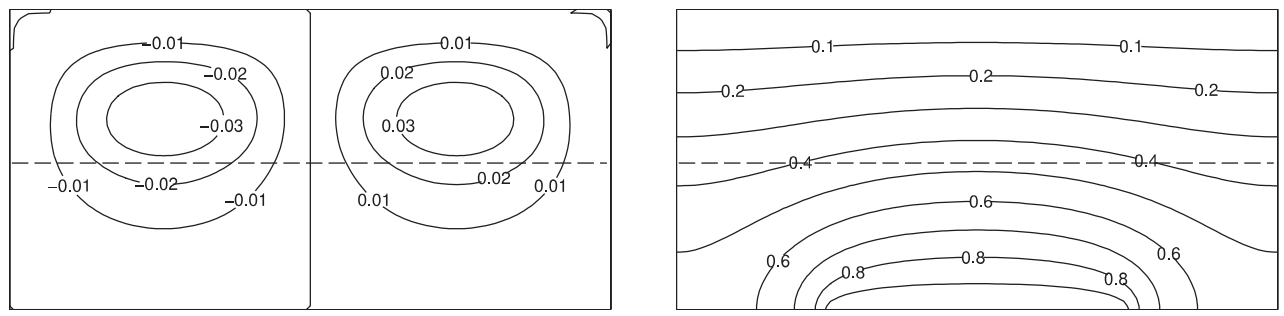

(b)
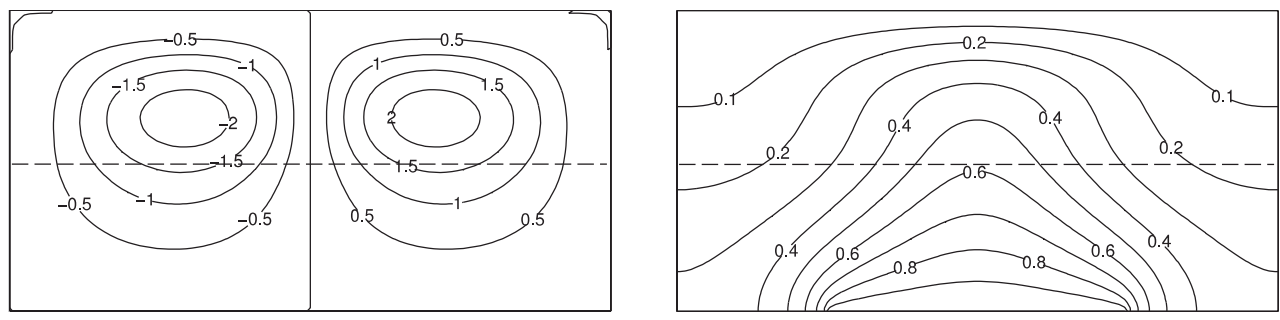

(c)
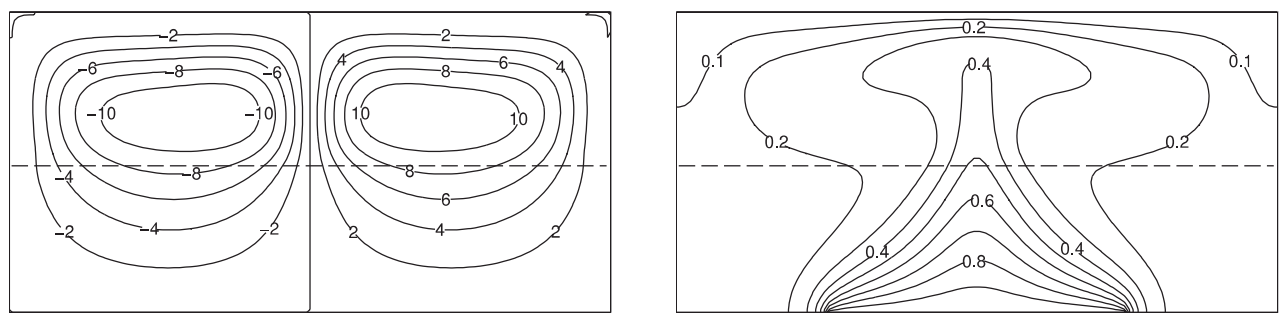

(d)
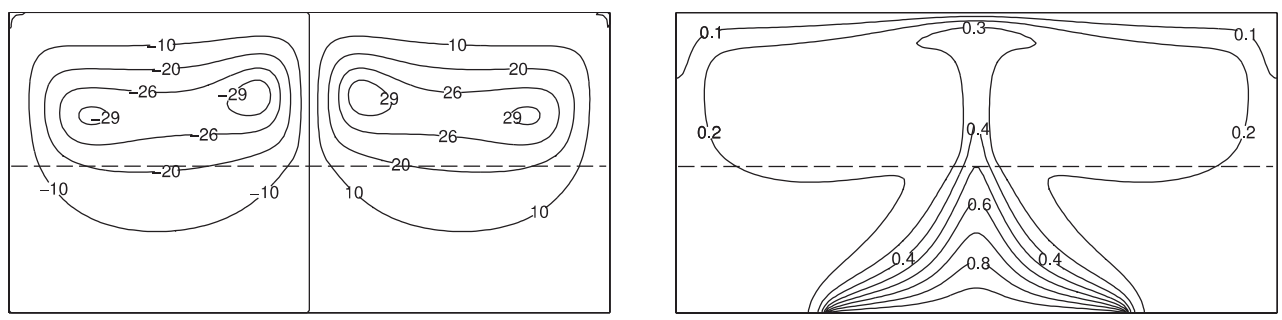

Figure 4.18: Streamline and isotherm patterns for convection in a composite layer for $D a=$ $10^{-6}, \delta=0.5$. (a) $R a=10^{3}$, (b) $R a=10^{4}$, (c) $R a=10^{5}$, and (d) $R a=10^{6}$.

are also applicable to alternative definitions of the Darcy number; numerical results for such definitions may be readily obtained from the present results by using equation (4.2), or a similar definition.

In the present study, three different Darcy numbers are considered: $10^{-6}, 10^{-4}$, and $10^{-2}$. As before, the discussion will begin with an analysis of the streamline and isotherm patterns, followed by a study of the Nusselt-Rayleigh number relationships. In addition, the case of $D a=10^{-6}$ will not be discussed in detail as this has already been extensively analyzed in the previous two sections. Instead, this case will be considered to be the "benchmark" and the discussion will follow the comparison of the other two cases with this case. Figures 4.18, 4.19 and 4.20 show the streamline and isotherm patterns at $D a=10^{-6}, 10^{-4}$, and $10^{-2}$ at four different Rayleigh numbers. Comparing the flow 
(a)

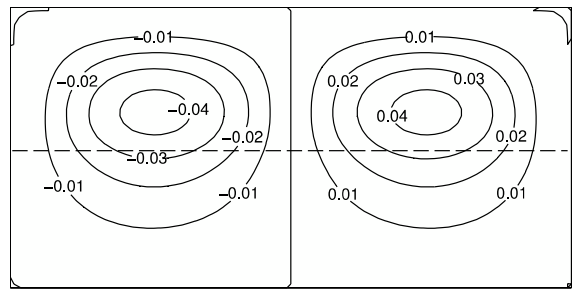

(b)

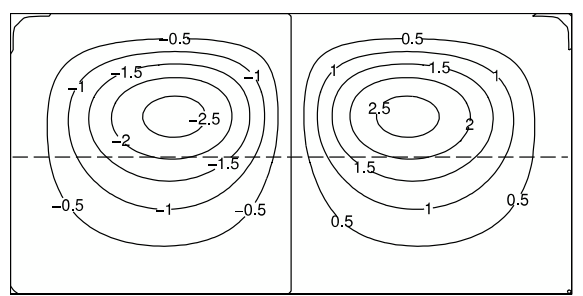

(c)

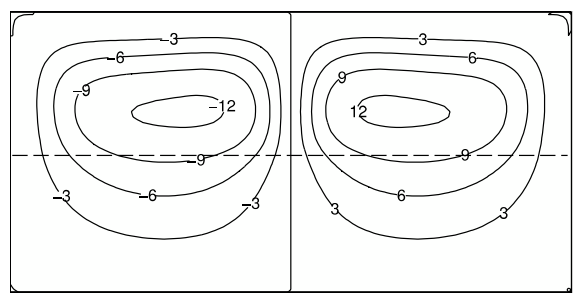

(d)

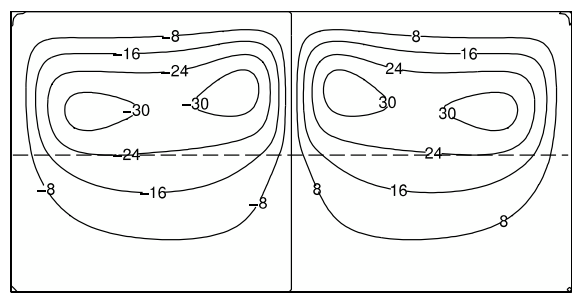

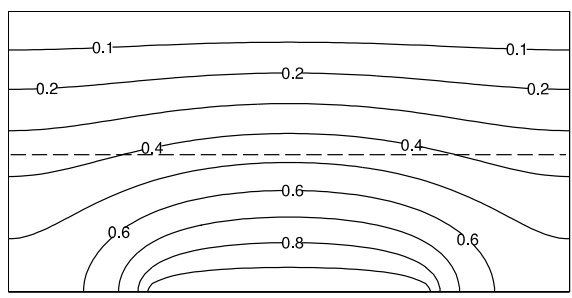
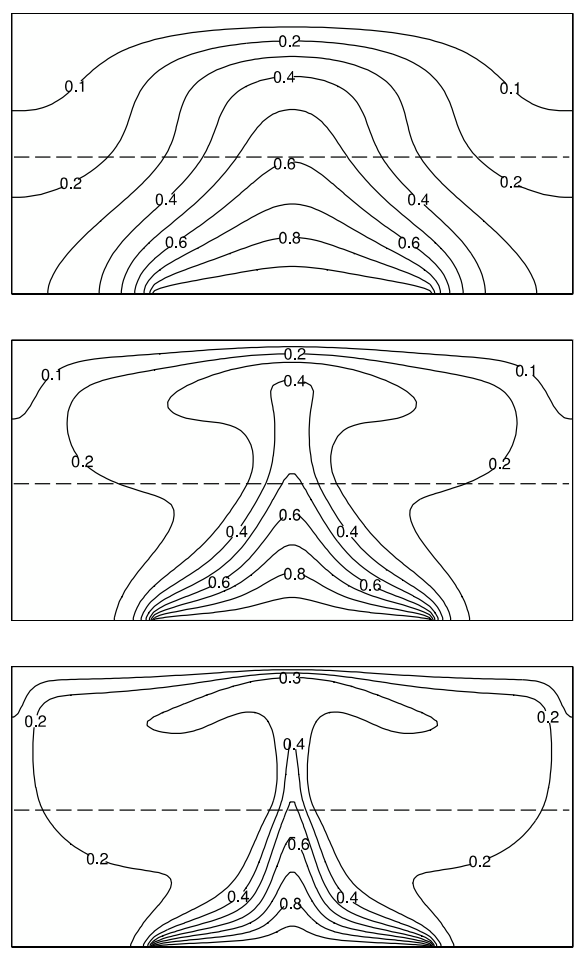

Figure 4.19: Streamline and isotherm patterns for convection in a composite layer for $D a=$ $10^{-4}, \delta=0.5$. (a) $R a=10^{3}$, (b) $R a=10^{4}$, (c) $R a=10^{5}$, and (d) $R a=10^{6}$.

patterns for $D a=10^{-6}$, and $10^{-4}$ it can be seen that there is not much difference between them. At $R a=10^{3}$, the localized heater driven convection patterns can be seen. The fluid velocities for $D a=10^{-4}$ are slightly higher than those for $10^{-6}$ as indicated by the slight difference in the value of the stream function. This, trend, is expected as a porous medium with a higher Darcy number offers lower resistance to fluid motion, and hence allows for higher flow velocities. Isotherm patterns, however, show that heat transfer is still conduction dominated at this value of $R a$.

With an increase in the value of the Rayleigh number to $10^{4}$, a convection dominated flow pattern can be seen for both values of the Darcy number. Fluid velocities increase and a pluming pattern can be seen to start forming in both cases. The values of the stream function indicate that flow velocities are almost identical in both cases. This is also the case when the Rayleigh number is increased to $10^{5}$. The flow and temperature fields are 
(a)
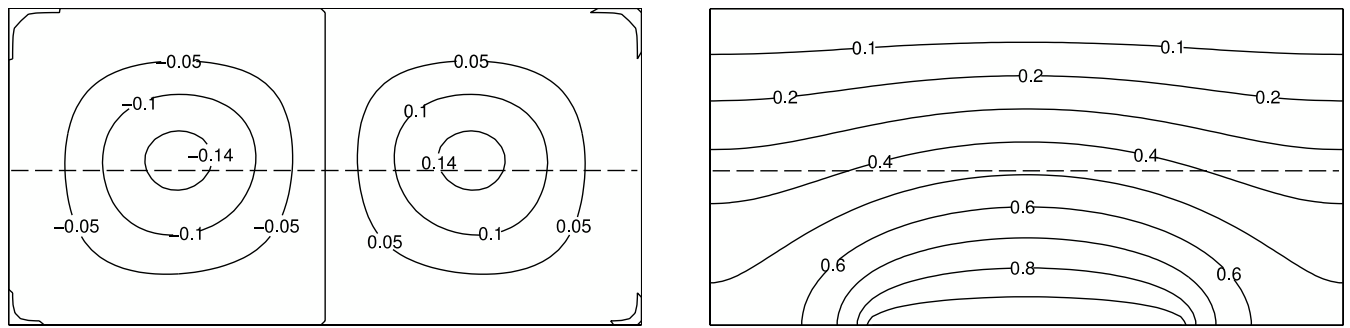

(b)
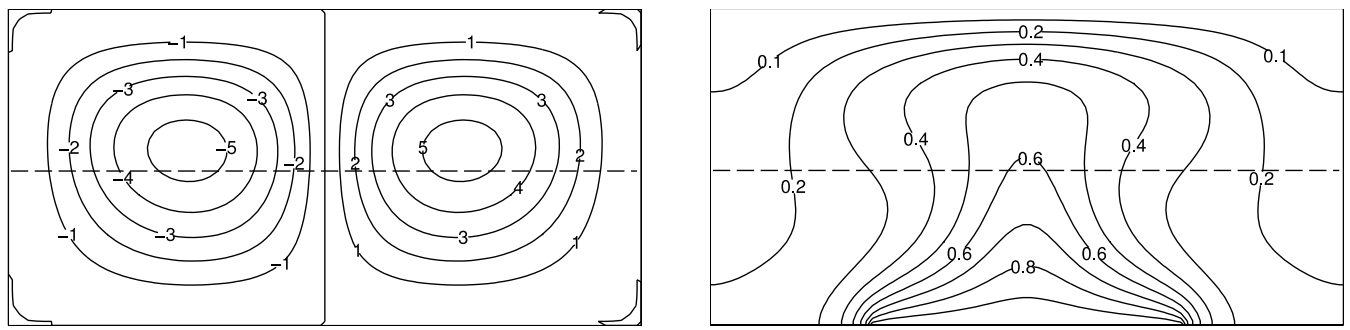

(c)
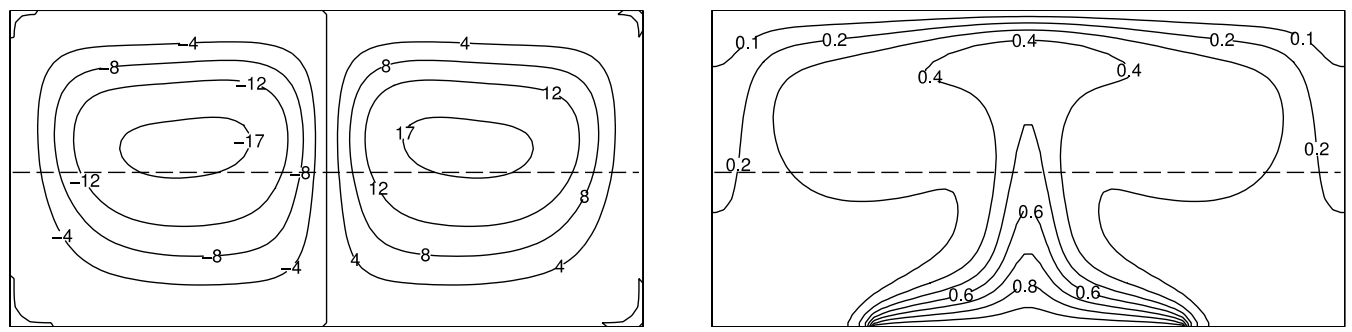

(d)
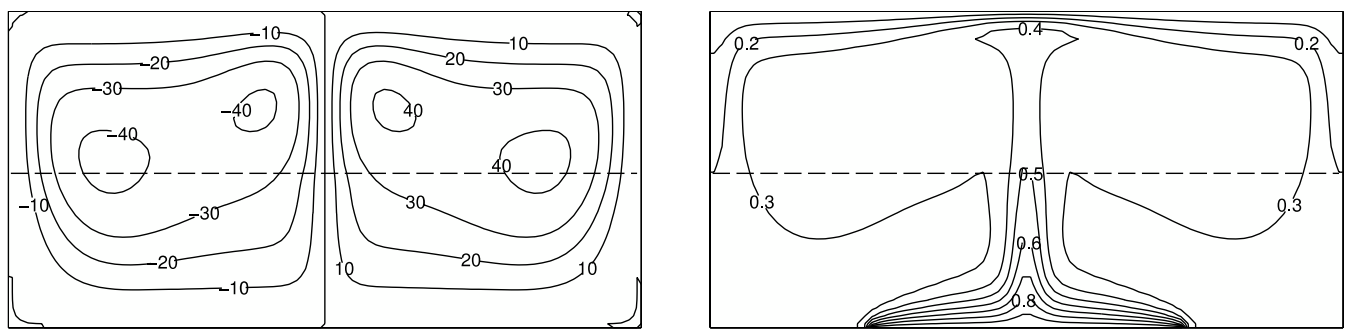

Figure 4.20: Streamline and isotherm patterns for convection in a composite layer for $D a=$ $10^{-2}, \delta=0.5$. (a) $R a=10^{3}$, (b) $R a=10^{4}$, (c) $R a=10^{5}$, and (d) $R a=10^{6}$.

virtually identical indicating that an increase in the Darcy number from $10^{-6}$ to $10^{-4}$ has little effect on the overall flow patterns, and hence, the heat transfer coefficients. When the Rayleigh number increases to $10^{6}$, however, there is a noticeable difference between the two cases. The streamlines show that while the flow velocities are comparable there is increased penetration of the flow into the underlying porous layer for $D a=10^{-4}$. The isotherm patterns also show that the average temperatures in the fluid layer increase with an increase in the Darcy number. These observations point to the fact that significant differences in flow patterns, and as a consequence, overall heat transfer rates between these two cases can be seen only at very high Rayleigh numbers.

When the Darcy number is further increased to $10^{-2}$, significant changes can be 


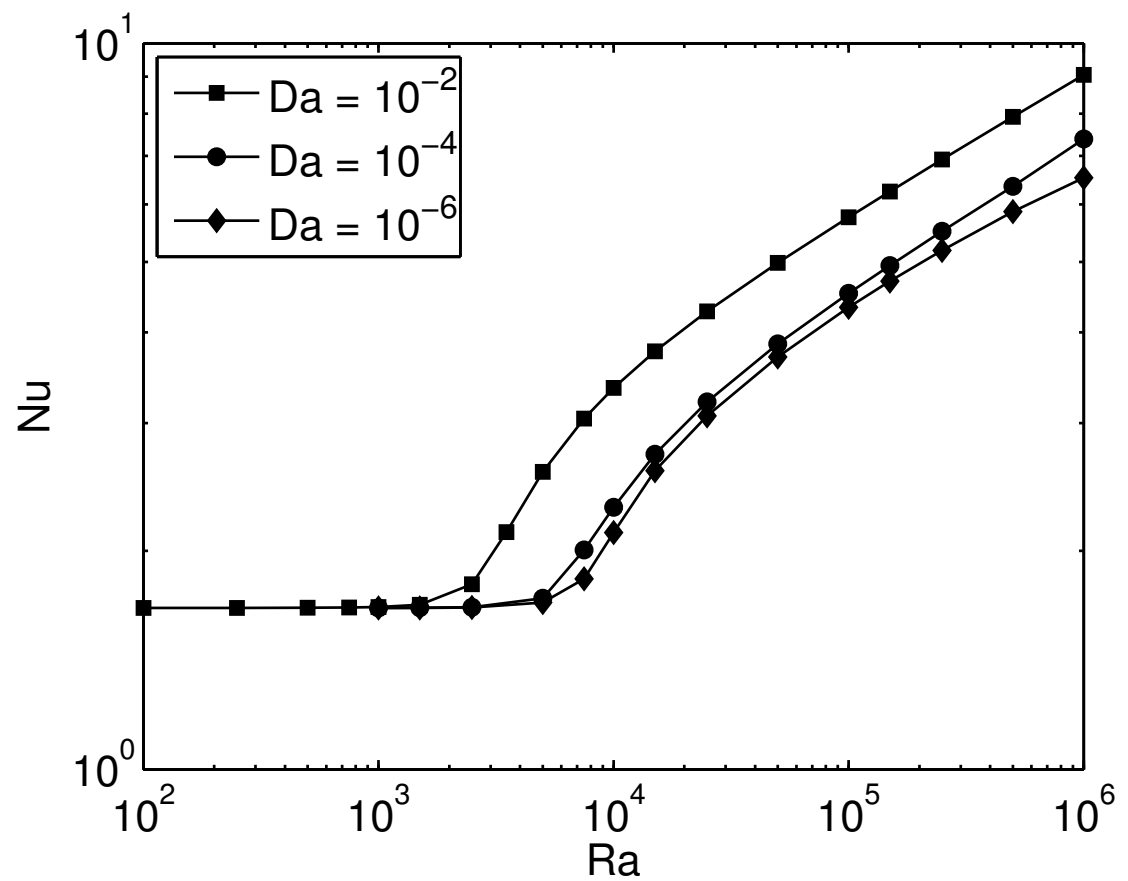

Figure 4.21: Nusselt-versus-Rayleigh number relationship for different Darcy numbers. $\delta=$ 0.5 and $\eta=0.5$.

seen. The most important one is that the flow is now no longer confined to the upper fluid layer, but is instead spread out across the two layers. This is a direct consequence of the increased permeability of the porous layer which allows much higher levels of flow penetration. The higher value of the Darcy number also means that the porous layer offers minimal resistance to convective flow which leads to higher flow velocities. With an increase in the Rayleigh number, the intensity of the convective motion increases and the convective cells acquire an almost square shape, indicating that convective motion in the fluid and porous layers is comparable. In fact the flow patterns at $R a=10^{6}$ bear a great resemblance to those seen in the Rayleigh-Bénard connection. Further insight can be gained by looking at the isotherm patterns. At $R a=10^{3}$, the isotherms show that the system is still in the conduction mode. At $R a=10^{4}$, a plume like flow is seen which is much more developed as compared to the isotherms for lower Darcy number flows. Interestingly, the thermal plume has an almost uniform character throughout the height of the cavity whereas at lower Darcy numbers, the plume had different characteristics in the fluid and porous regions. Thus it can be deduced that the temperature profile along the plume will be significantly different from the profiles for lower Darcy numbers. This aspect will be discussed shortly.

The overall heat transfer results for different Darcy numbers are shown in Figure 4.21. As can be seen, the Darcy number has an effect on both the onset of convection as well as the overall heat transfer coefficients beyond the critical point. As the Darcy number 


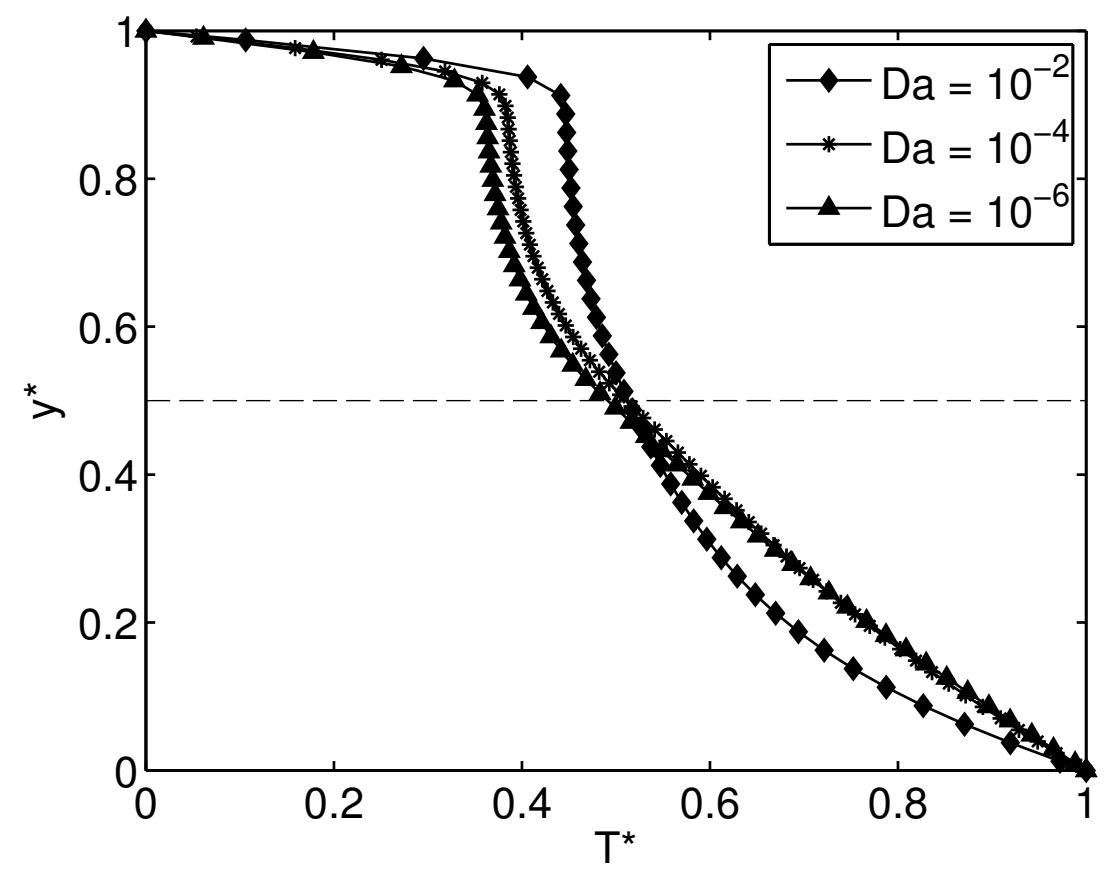

Figure 4.22: Dimensionless temperature $\left(T^{*}=T-T_{C} / T_{H}-T_{C}\right)$ profiles along the vertical centerline of the domain for different Darcy numbers. $\delta=0.5, \eta=0.5$ and $R a=5 \times 10^{5}$.

increase from $10^{-6}$ to $10^{-4}$, the critical Rayleigh number where convection takes over as the dominant heat transfer mode decreases slightly, although this is not very clearly visible. With further increase in $D a$, however, the critical Rayleigh number drops noticeably. The value of the Nusselt number in the conduction dominated heat transfer regime however is the same for all Darcy numbers as the heater length is kept fixed. Once convection starts dominating, the heat Nusselt numbers for $D a=10^{-6}$ and $10^{-4}$ are very close to each other except at very high Rayleigh numbers where the effects of lower permeability can be seen. It is likely that this effect is due to the fact that inertial effects start dominating the flow. The heat transfer coefficients for $D a=10^{-2}$ are, however, much higher than those at lower Darcy numbers. This result is expected based on the prior discussion of the flow patterns which shows that high levels of convective penetration into, and reduced resistance to fluid motion by, the underlying porous layer leads to increased heat transfer. The dimensionless temperature profiles along the centerline of the cavity (Figure 4.22) also indicate that the temperature distribution for $D a=10^{-2}$ is significantly different from those Darcy numbers. This is especially true for the temperatures within the porous layer and clearly shows that the porous layer at such high values of the Darcy number has an almost fluid like character. These results may be of high importance for applications where the porous layer is loosely packed. 
(a)
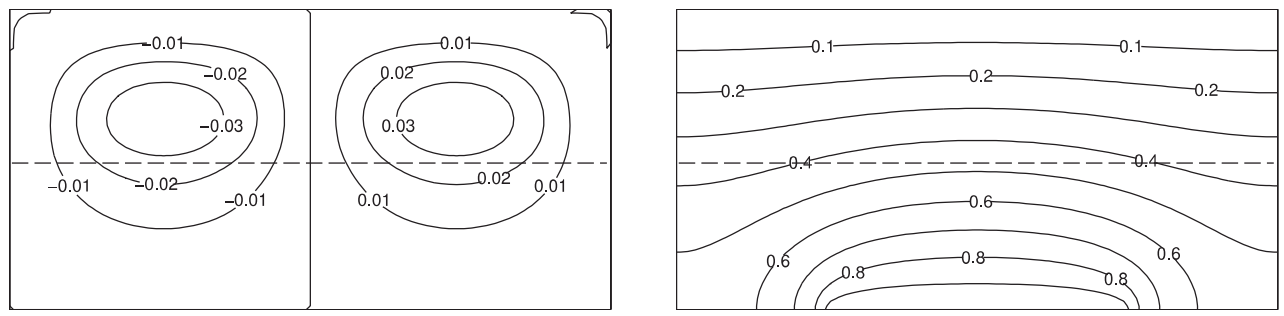

(b)
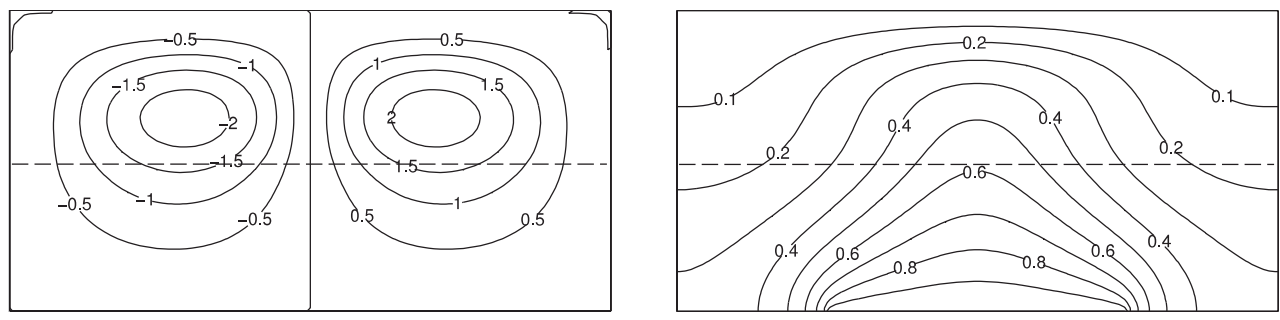

(c)
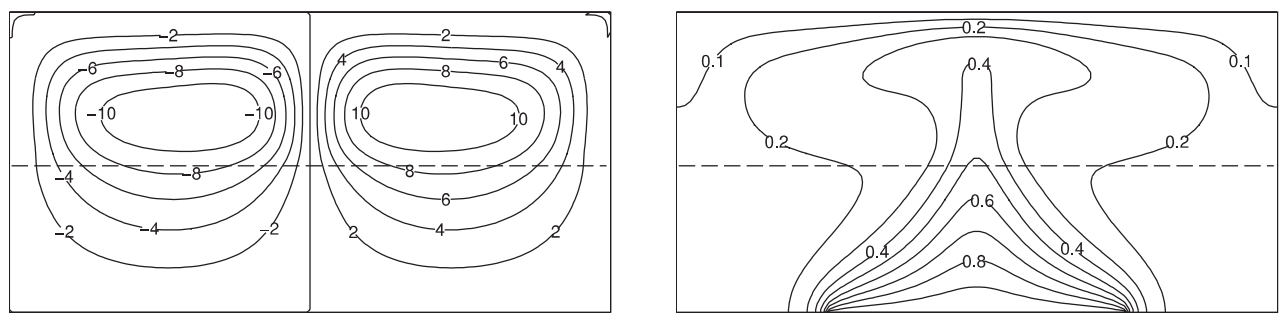

(d)
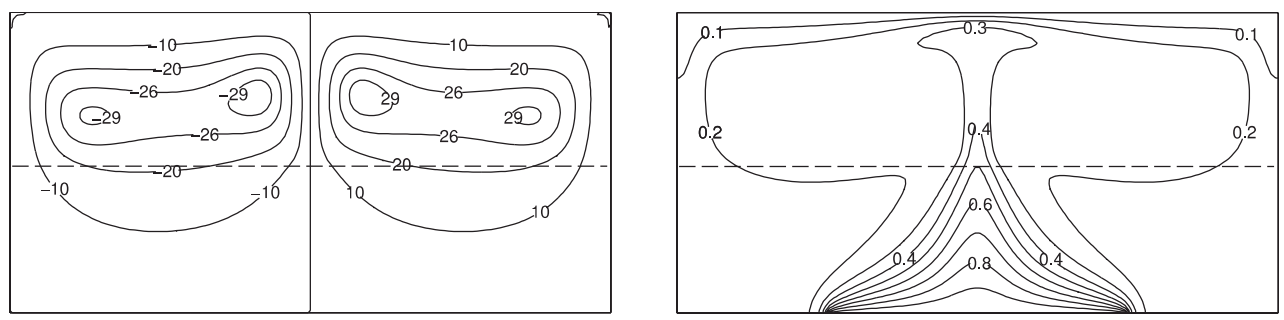

Figure 4.23: Streamline and isotherm patterns for convection in a composite layer for $A=2$, $\delta=0.5$. (a) $R a=10^{3}$, (b) $R a=10^{4}$, (c) $R a=10^{5}$, and (d) $R a=10^{6}$.

\subsubsection{Effect of the aspect ratio}

The aspect ratio is a very important parameter in determining the overall heat transfer rates, especially when the heat source is localized. Prior investigations of convection in fluid and porous layers with localized heat sources have shown that there is a complex relation between the aspect ratio and the heater length. It is therefore worthwhile to investigate whether such a relation exists for convection in fluid superposed porous layers. In the present study, three different aspect ratios are considered: $A=2,4$, and 6 . Figures $4.23,4.24$, and 4.25 show the streamline and isotherm patterns at four different Rayleigh numbers for aspect ratios $A=2,4$, and 6 respectively. The parameters kept constant in these simulations are $\eta=0.5, D a=10^{-6}, \lambda=1$ and $P r=7$. From 4.23(a) and 4.24(a) it can be seen that the aspect ratio does not have any significant influence on the flow 
(a)
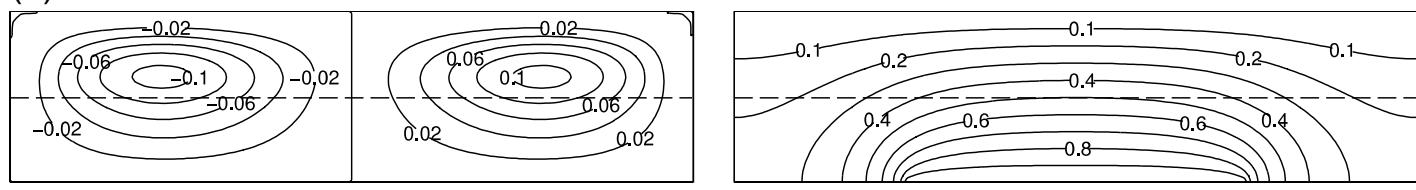

(b)
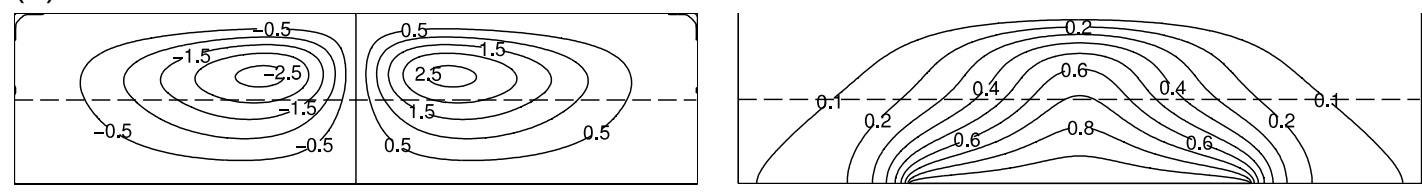

(c)
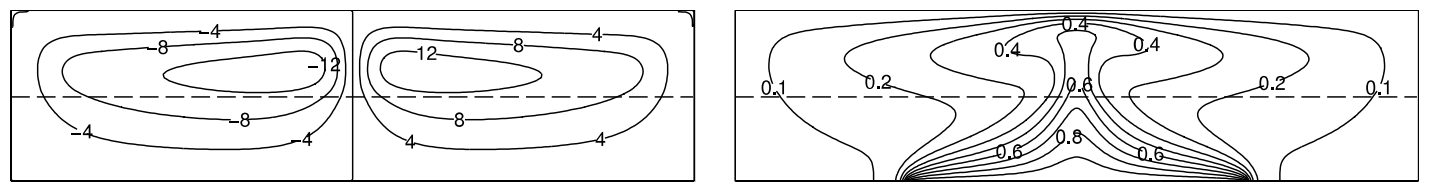

(d)
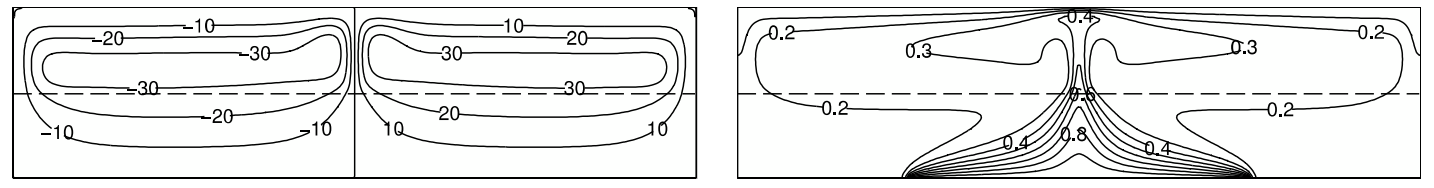

Figure 4.24: Streamline and isotherm patterns for convection in a composite layer for $A=4$, $\delta=0.5$. (a) $R a=10^{3}$, (b) $R a=10^{4}$, (c) $R a=10^{5}$, and (d) $R a=10^{6}$.

and temperature fields at $R a=10^{3}$. The circulating flow triggered by the heater-edge temperature gradient can be seen for both aspect ratios. Interestingly, the cells for $A=4$ are far apart from each other and are almost centered on the vertical lines drawn at the edges of the heater, further indicating their origin point. This feature cannot be seen at lower aspect ratios owing to the smaller horizontal extent of the domain. Also, the velocity of the convective motion is slightly higher at $A=4$. The corresponding isotherms, however, indicate that convection dominated heat transfer has not yet commenced in either system. At $R a=10^{4}$, convection dominated heat transfer can be seen for both aspect ratios. It can be seen that for $A=4$, the shape and the position of the circulating cells changes once convective flow is underway. The cells move away from their original positions and towards each other. This happens because the cells are associated with the thermal plume and therefore move towards the center of the heater to align themselves with the developing convective flow. The flow, however, remains confined to the upper fluid layer. As the Rayleigh number increases further to $10^{5}$, the cells stretch outwards towards the lateral walls. This behavior can be more prominently seen for the larger aspect ratio domain. The values of the stream function however have almost the same values for both aspect ratios indicating that the flow velocities are comparable in the two cases. The isotherm patterns 


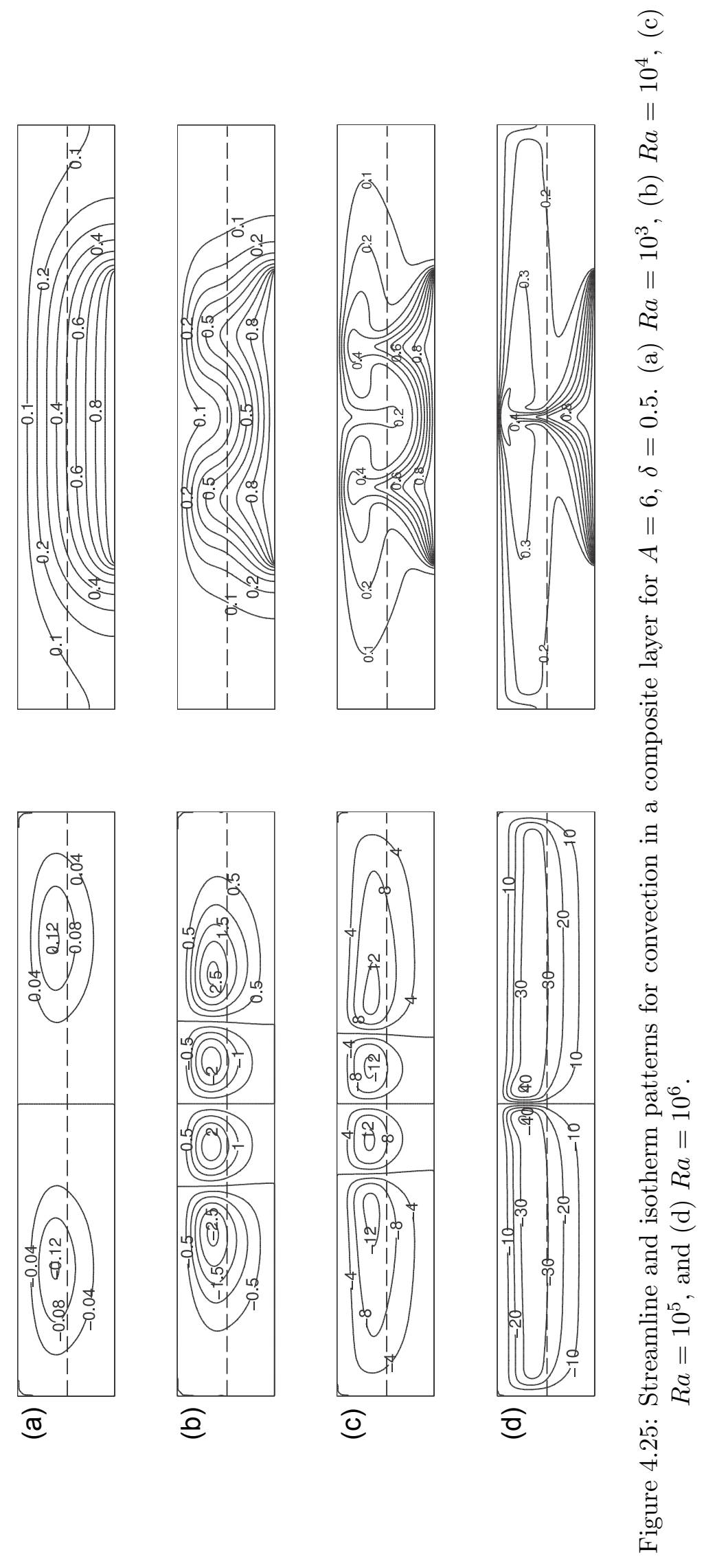


show that the rising thermal plume becomes narrower and takes on a well defined shape in both the long and short domains. Further increase in the Rayleigh number causes the circulating cells to stretch further and the thermal plume to become narrower.

An interesting aspect of the flow is that the number of circulating cells does not increase with an increase in the aspect ratio. This is in contrast to the case of a uniformly heated base where the wavelength and hence the number of the convective cells is determined by the aspect ratio of the domain. Thus for a domain with $A=4$, there would be four circulating cells when the base is uniformly heated. However when the heating is localized, the number of cells is a function of both the aspect ratio and the heater-to-base length ratio. This phenomenon has been observed in studies on convection in porous layers with localized heating by Elder [29] and Prasad and Kulacki $[57,58]$. Thus in the present case the values of $\delta$ and $A$ are such that only two cells are observed.

When the aspect ratio increases to $A=6$, several interesting flow patterns are seen. At $R a=10^{3}$, circulatory cells triggered by the heater edge temperature gradient can be seen. As for lower aspect ratios, the flow is conduction dominated which can be clearly seen from the isotherm patterns. With an increase in the Rayleigh number to $10^{4}$, convection dominated flow commences and two pairs of circulating cells are seen. This is a further example of how the number of cells depends on both the heater length and the aspect ratio. Within each pair of cells, there is, however, a conspicuous lack of symmetry; the cells towards the center of the domain are smaller while those near the lateral walls are larger. This is primarily a consequence of the readjustment of the size and position of the cells due the particular combination of the heater length and aspect ratio. With further increase in the Rayleigh number to $10^{5}$, the same flow pattern is seen with higher flow velocities. Also, the cells near the lateral walls stretch further horizontally to cover the entire domain. The isotherm patterns show two thermal plumes, each associated with one pair of convective cells. When $R a=10^{6}$, however, a very interesting phenomenon occurs. The cells in each circulating pair merge to create a single pair of cells which span the entire domain. The isotherm patterns now show a single narrow rising plume reflecting the flow restructuring. This readjustment occurs at around $R a=5 \times 10^{5}$. This phenomenon is very unique and is not seen when the base is uniformly heated. It seems that the origin of this phenomenon lies in the asymmetry of the flow structure that was noted earlier and due to this asymmetry the cells near the vertical walls keep growing laterally while the inner cells remain fixed in size. When these outer cells cannot grow further outward, they start growing inward and swallow up the inner cells to give rise to a two cell flow structure.

To further illustrate this point, consider the above case of $A=6$ but with a heater length fraction $\delta=0.75$ instead of $\delta=0.5$. The streamlines and isotherms for this configuration are shown in Figure 4.26. At $R a=10^{4}$, three pairs of circulating cells are seen, 


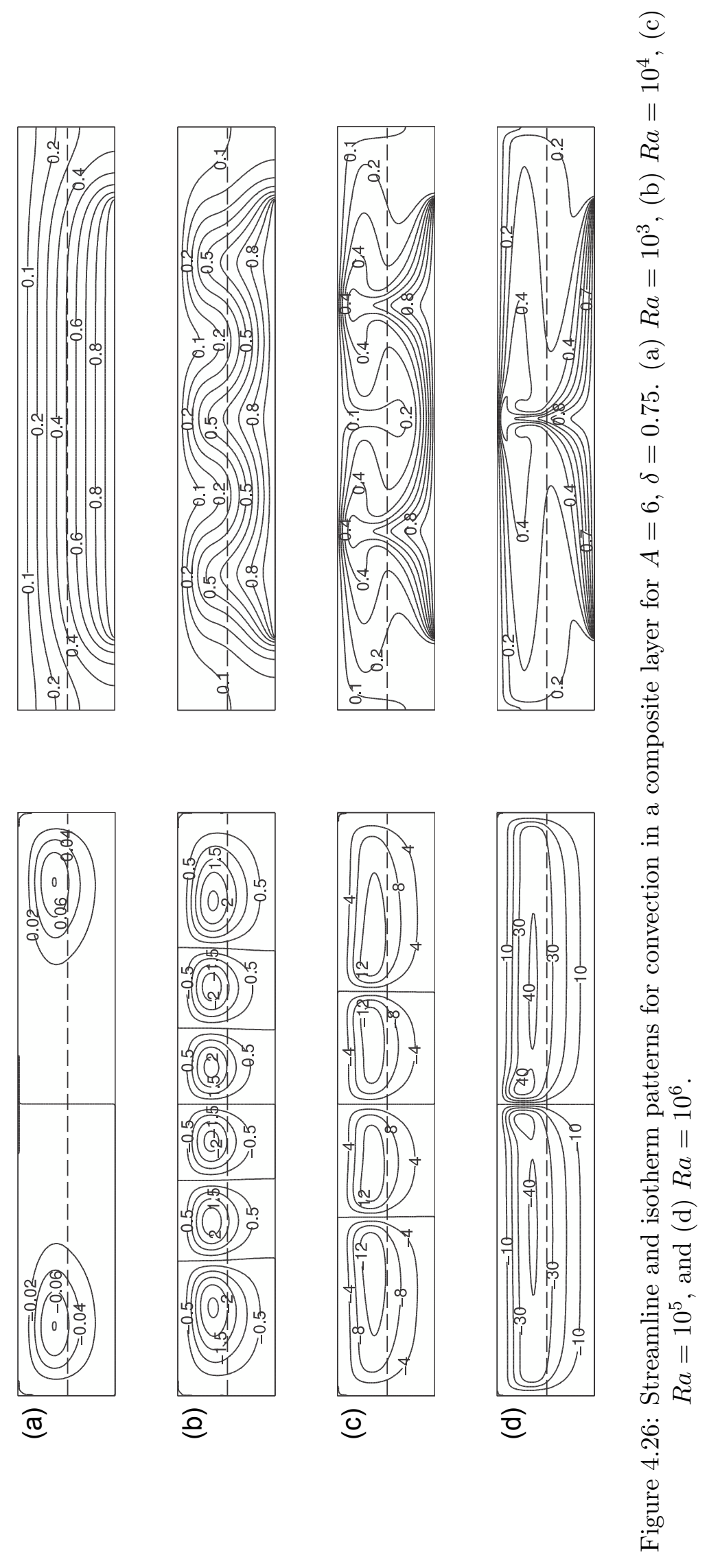


the same number of cells that would be present if the base were uniformly heated. As seen before, due to the presence of a local heat source, the outermost pairs of cells are asymmetrical. As a result, when the Rayleigh number is increased to $10^{5}$, restructuring of the flow takes place and two pairs of circulating cells are formed. However, the asymmetry in the system still persists. Thus further increase in the Rayleigh number leads to further flow restructuring, and at $R a=10^{6}$, a single pair of circulating cells remain. These cells span the entire horizontal extent of the cavity although the largest fluid velocities are in the overlying fluid layer. The isotherm patterns show that with increase in the Rayleigh number, the number of thermal plumes decreases progressively. These results show that when the heat source at the base is localized, the number of circulating cells is a function of the aspect ratio, heater length ratio, and the Rayleigh number. This phenomenon has not been observed in prior studies on convection in horizontal fluid or porous layers heated locally from below. Poulikakos [21] has reported the occurrence of flow restructuring at high Rayleigh numbers in fluid-superposed porous layers heated uniformly. However, no such phenomenon was observed in the present study for $\delta=1$. It may thus well be that such rearrangement of convection patterns is an artifact of the numerical solution although it is impossible to say this at the present with any degree of certainty. Thus, this aspect needs to be explored further experimentally.

The Nusselt-versus-Rayleigh number relation for different aspect ratios is shown in Figure 4.27. As can be seen, the curves for all aspect ratios have the same pattern across the entire range of Rayleigh numbers although the overall heat transfer rate decreases with an increase in the aspect ratio. In each case, however, the critical Rayleigh number for the onset of convection dominated heat transfer is approximately the same. The curves also show several interesting features which are a direct consequence of the flow patterns discussed earlier. Looking at the curves for $A=2$ and 4 , it can be seen that beyond the onset of convection dominated flow, Nusselt numbers for $A=4$ increase at a higher rate than that for $A=2$ until $R a \approx 5 \times 10^{4}$, after which they remain approximately parallel indicating that the overall heat transfer rate increases at approximately the same rate for both cases. This can be explained by considering the flow patterns in the two systems. At low Rayleigh number, a fraction of the domain for $A=4$ does not have any fluid motion. However, the streamline patterns for $A=2$ indicate that fluid motion covers almost the entire horizontal extent of the domain. Hence the system with a lower aspect ratio is able to more efficiently transfer heat by convection. However, with increase in $R a$, the flow spreads further towards the side walls for the system with the larger aspect ratio and the stagnant fluid near the walls now participates in convection. For smaller aspect ratio domains, however, the flow cannot further spread laterally as it has already reached its maximum extent. As such, the heat transfer coefficient $A=4$ increases more rapidly. Once 


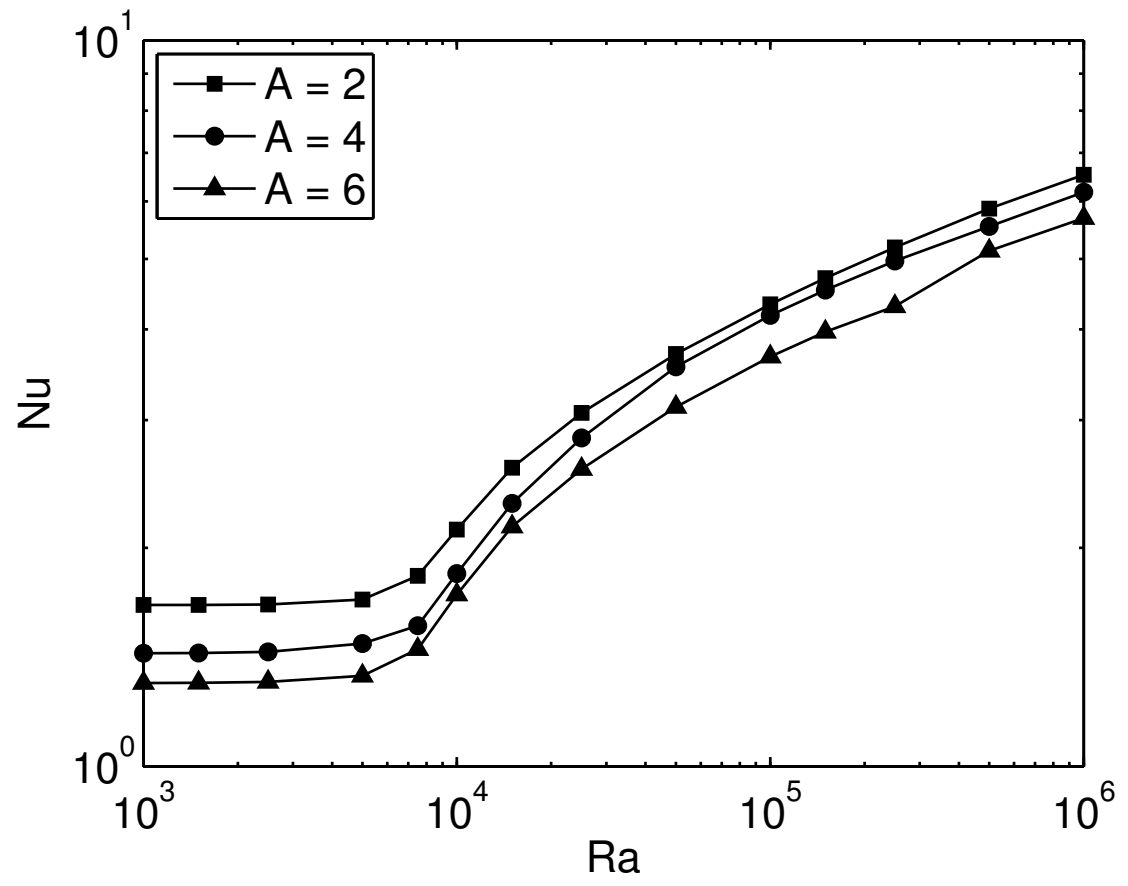

Figure 4.27: Nusselt-versus-Rayleigh number relationship for different aspect ratios. $\delta=$ 0.5 and $\eta=0.5$.

the flow has spread to its maximum lateral extent for the longer system, however, the rate of increase of the Nusselt number becomes identical for both the aspect ratios. For $A=6$, the curve is almost parallel to that of $A=2$ until $R a \approx 2.5 \times 10^{5}$. Beyond this Rayleigh number however there is an abrupt change in the slope of the curve, and Nusselt numbers beyond this point are much closer to those for lower aspect ratios. This is a direct consequence of the flow restructuring that creates a single pair of circulating cells. When the number of cells is larger, there are pockets of stagnant fluid between the cells which do not participate in convection. However, when the cells merge, all the fluid within the domain participates in convective motion leading to higher overall rates of heat transfer.

The dimensionless temperature profiles along the centerline of the cavity at $R a=10^{5}$ are shown in Figure 4.28. These profiles show some very interesting patterns. It can be seen that all along the cavity height, the centerline temperatures increase with the aspect ratio. This pattern is remarkably similar to the temperature centerline temperature profiles for different values of the heater length ratio $\delta$, shown earlier in Figure 4.12. It can be seen that for $A=2$, the temperature drops much more rapidly within the porous layer than for $A=4$ and 6 . This implies that the temperature gradient near the heater increases with a decrease in the aspect ratio. This leads to a more efficient channeling of the heat input from the heater and causes the lower aspect ratio domain to have higher heat transfer coefficients. 


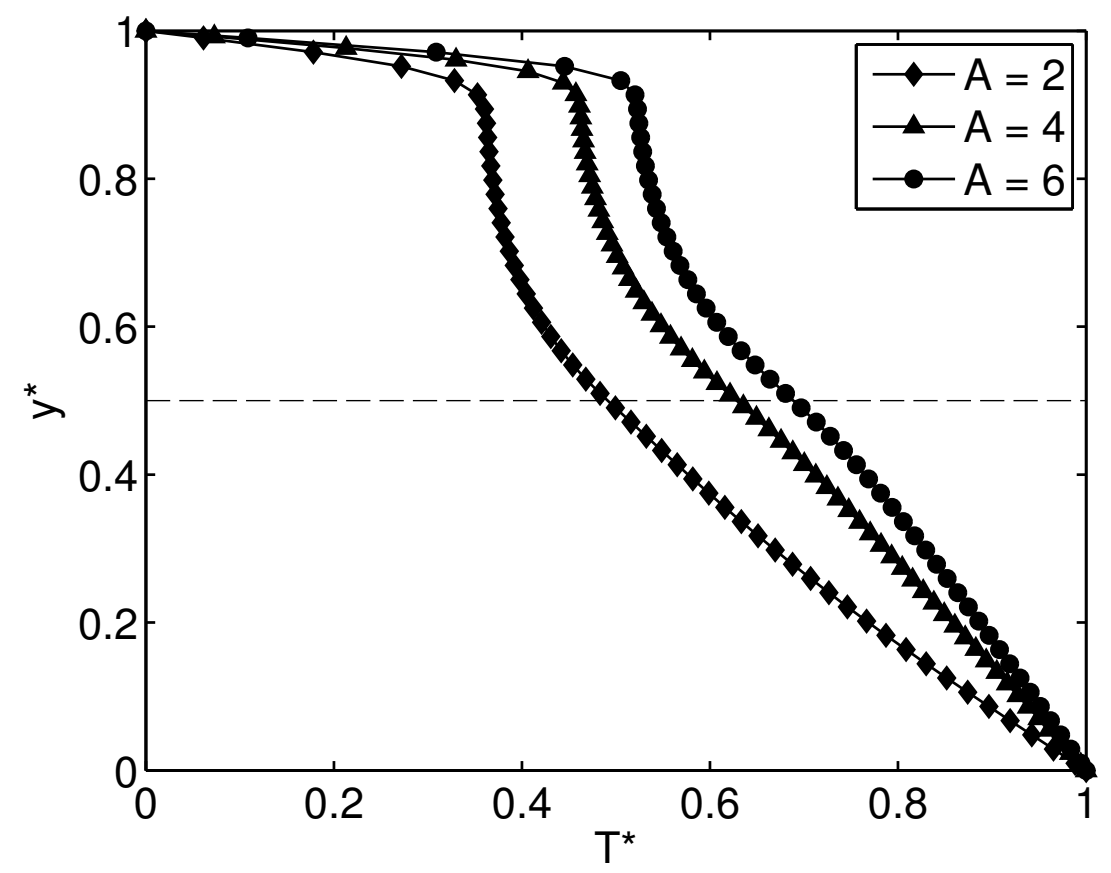

Figure 4.28: Dimensionless temperature $\left(T^{*}=T-T_{C} / T_{H}-T_{C}\right)$ profiles along the vertical centerline of the domain for different aspect ratios. $\delta=0.5, \eta=0.5$ and $R a=5 \times 10^{5}$.

\subsubsection{Effect of the solid-to-fluid conductivity ratio}

All simulation thus far have been carried out for the case where the conductivities of the solid and fluid phase are the same, i.e., $\lambda=1$. In reality, however, this condition is rarely satisfied, and many practical applications involve high solid-to-fluid conductivity ratios. Modeling of convection in porous media with high conductivity ratios, however, poses several challenges. First, a suitable model for the effective stagnant conductivity must be selected as improper representation of this parameter can significantly affect the overall results. Secondly, for very high conductivity ratios, the assumption of thermal equilibrium between the fluid and solid phases breaks down and, as a result, a single energy equation can no longer be used. Instead a two-equation model that accounts for the thermal resistance at the solid-fluid interface must be used which makes the numerical solution of the problem highly involved.

In order to systematically study the effect of the conductivity ratio it is important to look first look at the modeling of the effective conductivity. Aichlmayr and Kulacki [27] have shown that the effective conductivity data from various literature sources can be grouped primarily into three categories based on the conductivity ratio: small conductivity ratios $(1 \leq \lambda<10)$, intermediate conductivity ratios $(10 \leq \lambda<1000)$, and high conductivity ratios $(\lambda \geq 1000)$. They postulate that these ranges demarcate the relative importance of 
(a)
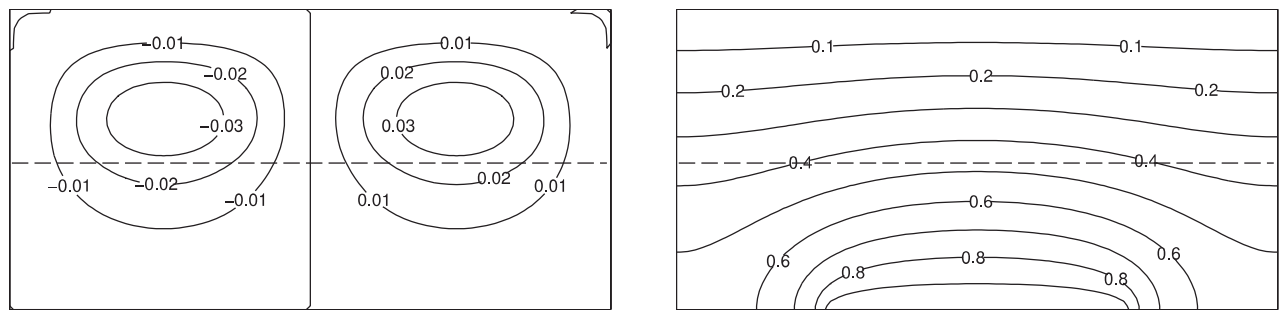

(b)
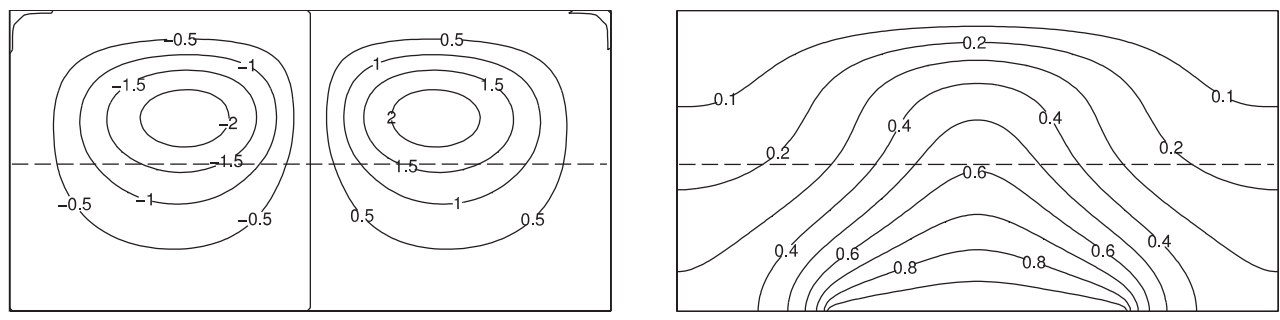

(c)
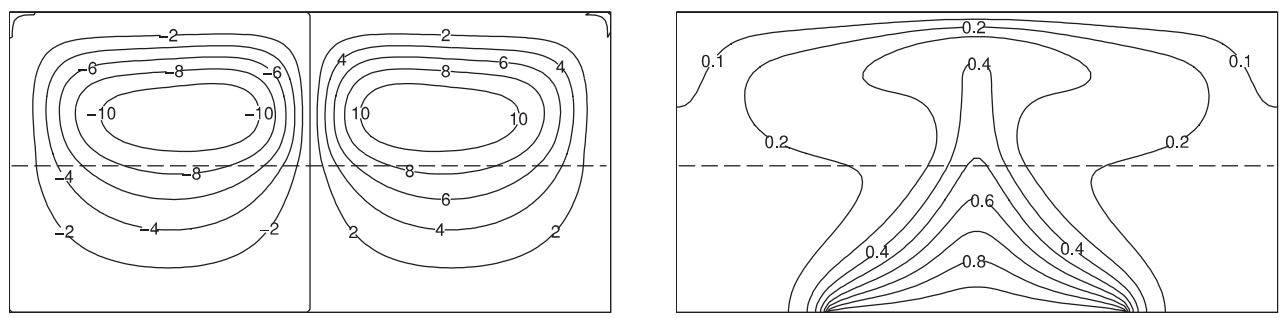

(d)
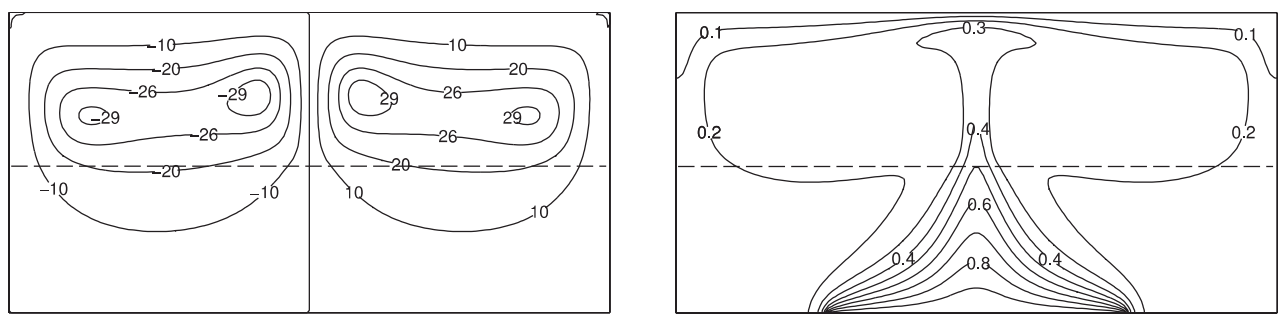

Figure 4.29: Streamline and isotherm patterns for convection in a composite layer for $\lambda=1$, $\delta=0.5$. (a) $R a=10^{3}$, (b) $R a=10^{4}$, (c) $R a=10^{5}$, and (d) $R a=10^{6}$.

the interfacial effects between the solid and fluid phases. High conductivity ratio systems typically have strong interfacial effects and, as a result, require the consideration of separate models for the solid and fluid phases. Owing to the difficulty of solving two-equation models, high conductivity ratio systems will not be considered here. Instead study will focus on low and intermediate conductivity ratios. Three different values of the conductivity ratio are considered here: $\lambda=1,50$, and 100. These values have been chosen based on the effective conductivities of glass-water $(\lambda=1.08)$, glass-air $(\lambda=48)$, and steel-water $(\lambda=$ 102) systems, all of which are very relevant for practical applications. To calculate the effective conductivity, two different models are used. For $\lambda=1$ the mixture model is used, while for $\lambda=50$ and $\lambda=100$, the model of Kunii and Smith [89] is used.

The streamline and isotherm patterns for $\lambda=1,50$, and 100 for different Rayleigh 
(a)

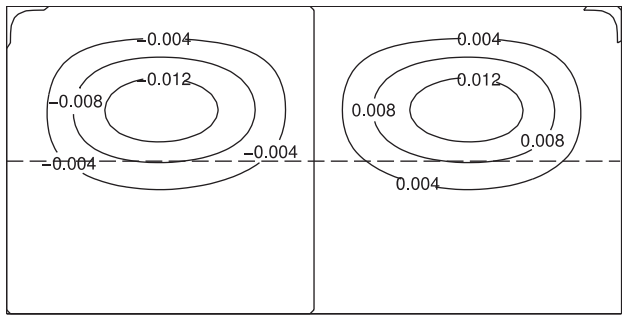

(b)

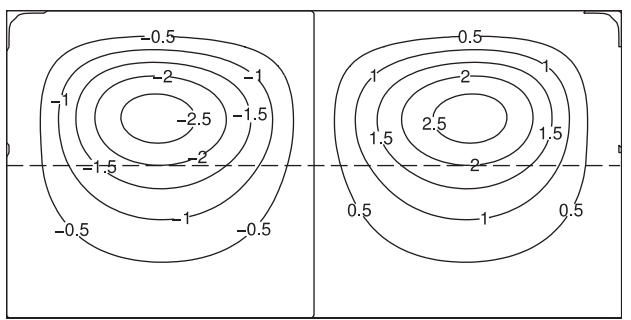

(c)

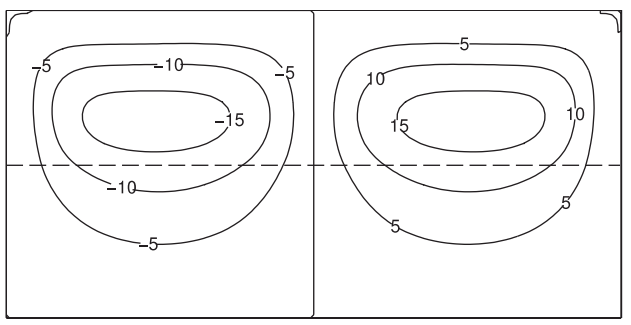

(d)

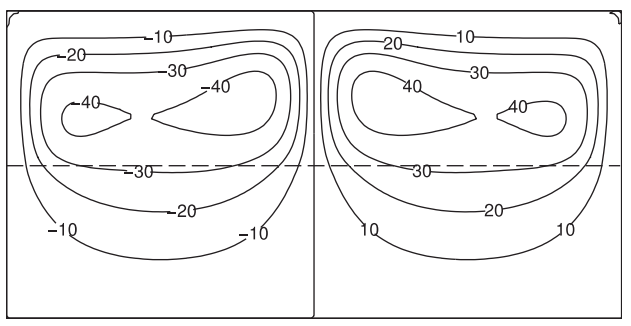

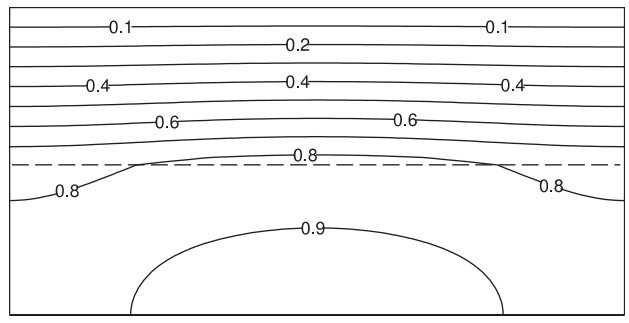
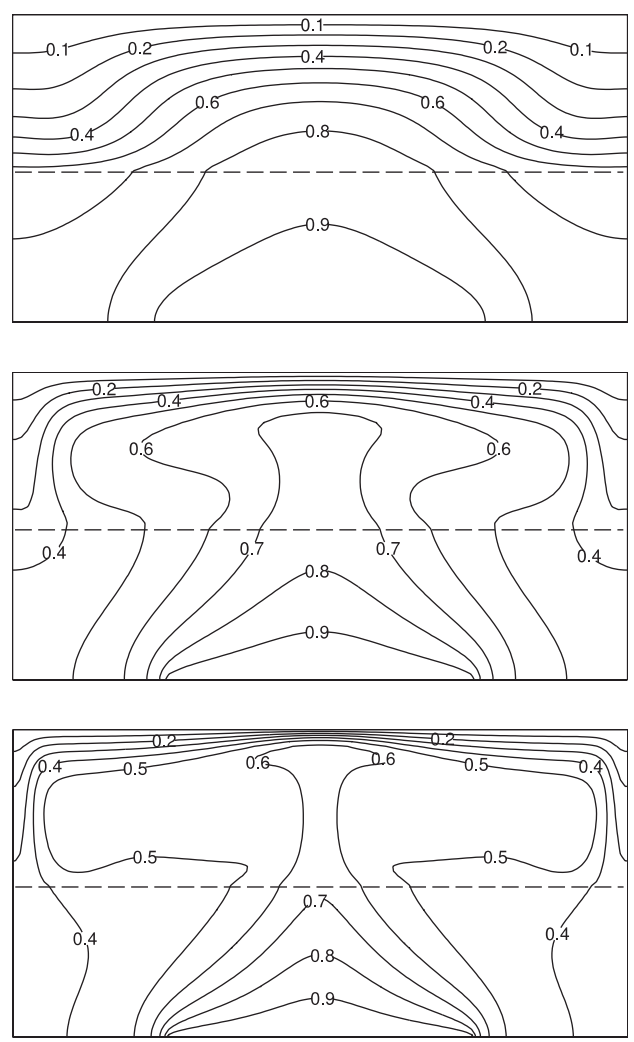

Figure 4.30: Streamline and isotherm patterns for convection in a composite layer for $\lambda=$ 50, $\delta=0.5$. (a) $R a=10^{3}$, (b) $R a=10^{4}$, (c) $R a=10^{5}$, and (d) $R a=10^{6}$.

numbers are shown in Figures 4.29, 4.30, and 4.31 respectively. In all these simulations the following parameters are fixed: $\delta=0.5, \eta=0.5, A=2, D a=10^{-6}$, and $\operatorname{Pr}=7$. The case of $\lambda=1$ has been discussed previously and will be discussed only with respect to the results for the other conductivity ratios. Comparing all the figures, it can be seen that there are significant differences in both the streamline and the isotherm patterns. As before, for $R a=10^{3}$, circulation patterns created by the presence of a localized heat source are seen in all cases and the heat transfer is primarily by conduction. The isotherm patterns, however, show significant differences. For $\lambda=1$, a gradual temperature change occurs across the entire domain. On the other hand, for $\lambda=50$ and $\lambda=100$ the temperature gradient across the porous layer is very small and the entire layer is approximately at a constant 
(a)

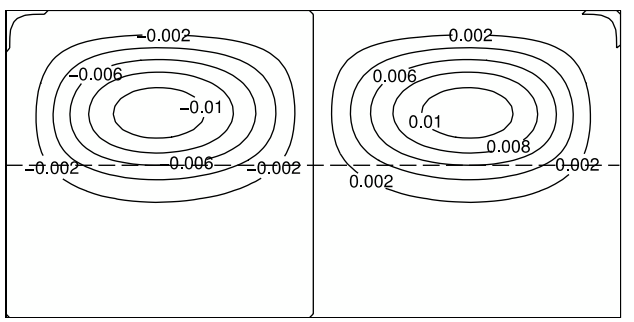

(b)

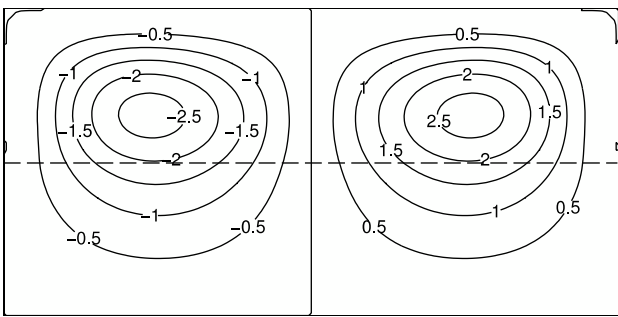

(c)

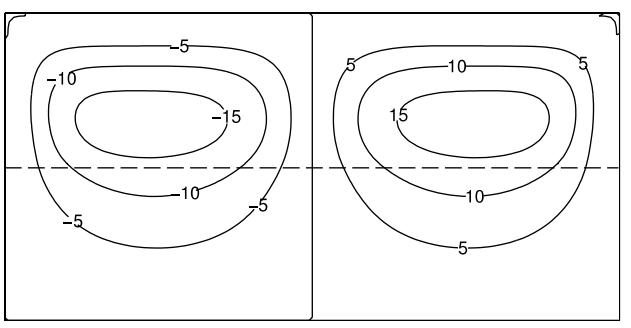

(d)

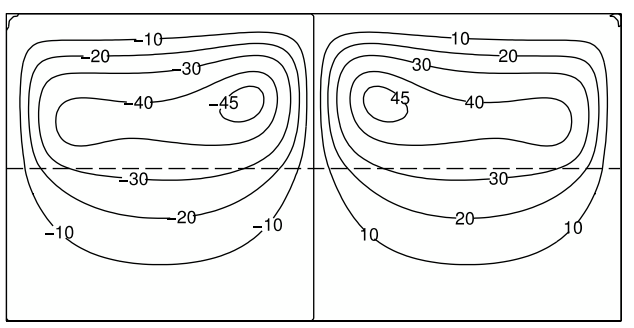

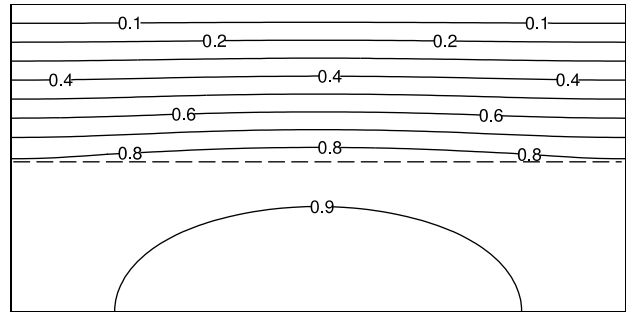
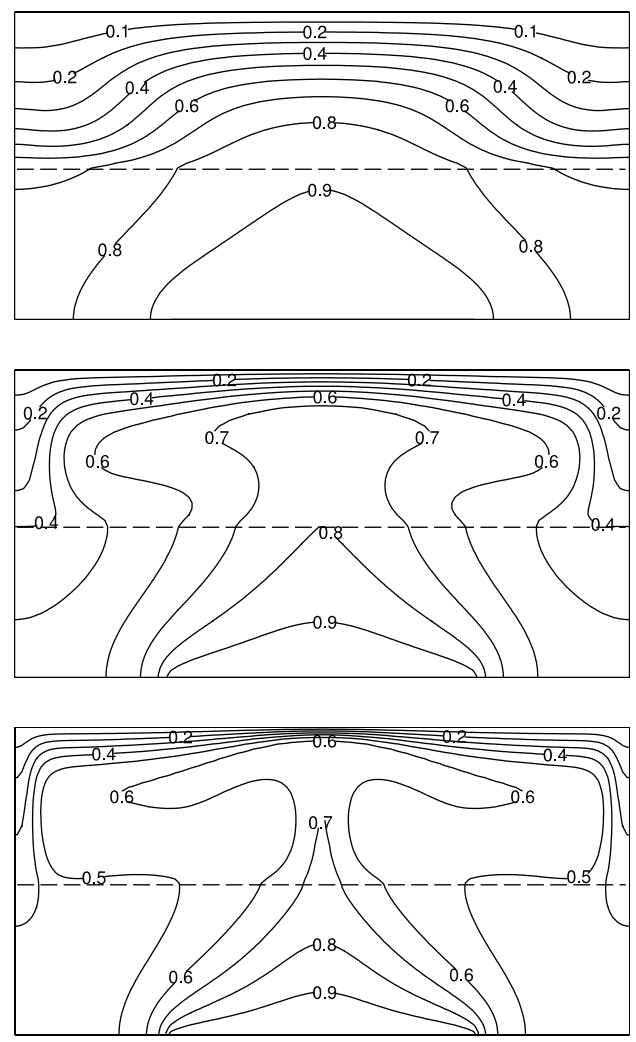

Figure 4.31: Streamline and isotherm patterns for convection in a composite layer for $\lambda=$ $100, \delta=0.5$. (a) $R a=10^{3}$, (b) $R a=10^{4}$, (c) $R a=10^{5}$, and (d) $R a=10^{6}$.

temperature. The major temperature drop takes place in the fluid layer indicating the presence of a high temperature gradient there. This is a direct consequence of the higher conductivity of the porous layer. Expressed differently, the saturated porous layer has a lower conduction resistance than the overlying fluid layer. As a result, a small temperature difference exists across the underling layer.

When the Rayleigh number is increased to $10^{4}$, convective flow patterns are seen in all the three cases. The flow patterns for $\lambda=1$ appear slightly different than that for $\lambda$ $=50$ and 100 in terms of the direction of the velocity vectors. Also, for $\lambda=1$, the flow is more prominent near the center of the domain whereas for $\lambda=50$ and 100, the flow is more prominent near the lateral walls of the cavity. The flow velocities in the former case are also 
slightly lower than velocities at higher conductivity ratios. Another interesting feature that can be seen is that flow penetration into the underlying porous layer is much higher for $\lambda$ $=50$ and 100. The isotherm patterns, however, differ significantly for the low $(\lambda=1)$ and high $(\lambda=50,100)$ conductivity ratios. For $\lambda=1$ a developing plume like pattern can be seen at $R a=10^{4}$. However, for $\lambda=50$ and 100, no clear plume like flow pattern is visible. Instead the isotherms, especially those in the fluid layer, indicate that heat transfer is still primarily by conduction. In addition the isotherms crossing the fluid-porous layer interface show an abrupt change in slope at the interface. This has been observed previously in studies on layered porous media by Lai and Kulacki [62] and is a direct consequence of the difference in conductivities of the two layers. The above observations also indicate that the overall flow and heat transfer patterns roughly follow the demarcation based on the effective conductivity: flow fields for $\lambda=50$ and 100 differ significantly from those for $\lambda=1$. As such, the remaining discussion will focus on analyzing results based the conductivity ratio regime, i.e., low $(\lambda=1)$ and high $(\lambda=50,100)$ instead of analyzing each case separately. This will help in clearly illustrating the effects of the conductivity ratio.

With further increase in the Rayleigh number to $10^{5}$, a well defined convective flow is seen in all the three cases. The streamlines indicate that the flow patterns in all the three cases are more or less identical. Interestingly, the flow velocities in the core of the convection cells are higher for the higher conductivity ratios. The isotherm patterns show a rising thermal plume for all conductivity ratios, although the plume for $\lambda=1$ is more developed than that seen at the higher conductivity ratios. Also, for the higher conductivity ratios, there is a very rapid drop in temperature from the top of the plume, to the upper isothermal surface indicating the presence of strong temperature gradients near the upper surface and thus much higher rates of heat transfer for the higher $\lambda$ values. This will be discussed further when the heat transfer characteristics and the temperature profiles in the cavity are analyzed.

Finally when the Rayleigh number increases to $10^{6}$, identical flow patterns are seen in all the three cases. Interestingly, the intensity of convective motion increases with an increase in the conductivity ratio as can be seen from the values of the stream function. Also, flow penetration from the overlying fluid later to the lower porous layer is higher for the higher conductivity ratios. The isotherms indicate a well formed rising thermal plume in all cases. As noted earlier, large temperature gradients exist near the top of the plume for the higher conductivity ratios. Interestingly, the abrupt changes in the isotherm gradients at the fluid-porous layer interface that are seen at lower Rayleigh numbers are much less pronounced than at higher Rayleigh numbers. This is another indication that convection is the dominant mechanism of heat transfer and helps to smooth out the effects of conductivity mismatch between the upper and lower layers. A significant effect of having 


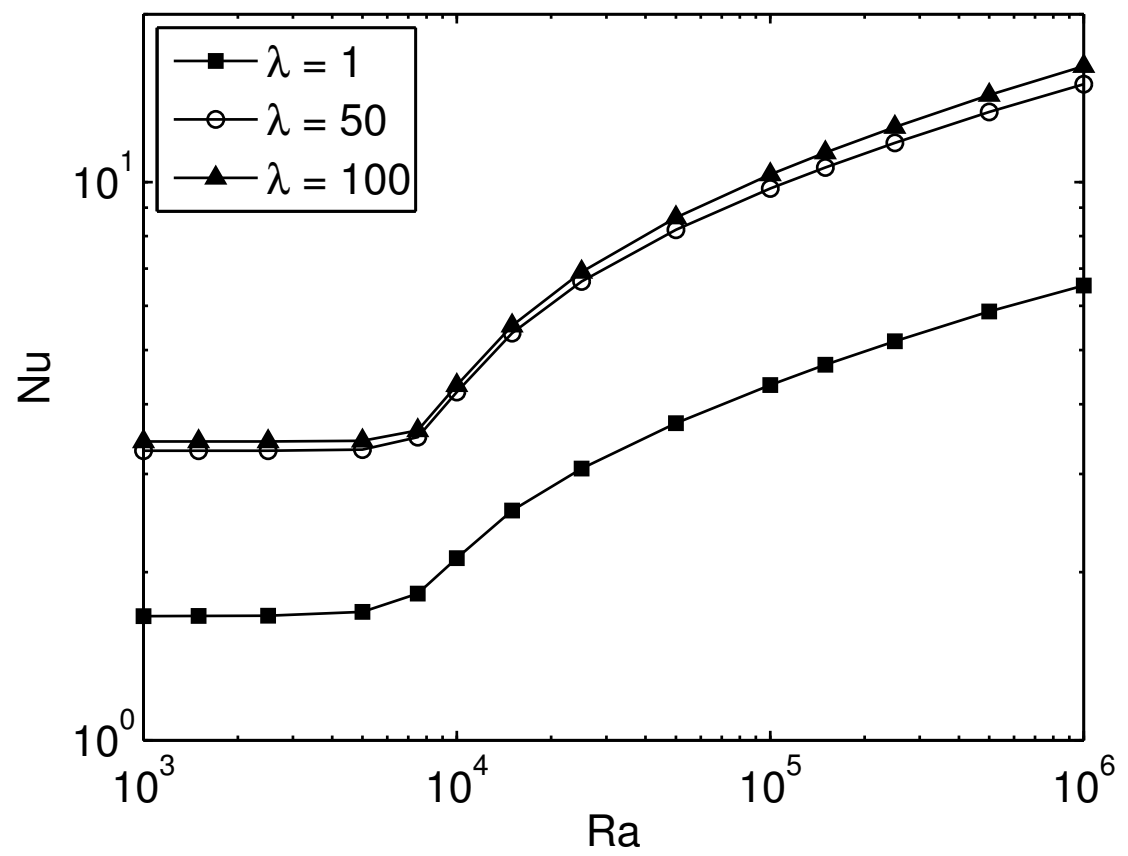

Figure 4.32: Nusselt-versus-Rayleigh number relationship for different conductivity ratios. $\delta=0.5$ and $\eta=0.5$.

a highly conductive porous layer is that the onset of convection dominated heat transfer occurs at higher Rayleigh numbers when compared to a less conductive matrix. This can be understood much better by comparing the heat transfer coefficients for the three systems at different Rayleigh numbers.

The Nusselt-versus-Rayleigh number relation for different conductivity ratios is shown in Figure 4.32. The figure clearly shows that an increase in the conductivity ratio increases the overall rates of heat transfer through the system as can be expected. A more interesting observation is that the curves for $\lambda=50$ and 100 are almost similar indicating that a twofold increase in the conductivity ratio does not significantly increase the overall heat transfer rates. This can be much better understood by considering the effective conductivity ratio, $\kappa$, for the two cases. For $\lambda=50, \kappa=6.1$ whereas for $\lambda=100, \kappa=7.5$. Because the effective conductivity is the independent parameter in the governing equations, the close value of $\kappa$ for the two cases is responsible for the almost same $N u-R a$ curves for them. Another interesting feature of the graphs is that the curves for $\lambda=1$ and $\lambda=50,100$ are not parallel to each other. This indicates that it is simply not the higher conductivity of the solid matrix which contributes to the higher heat transfer rates for higher conductivity ratios. As noted earlier, the intensity of the convective motion in the upper fluid layer, as well as the flow penetration in the underlying fluid layer, is higher at the higher conductivity ratios, which leads to higher overall heat transfer rates. 


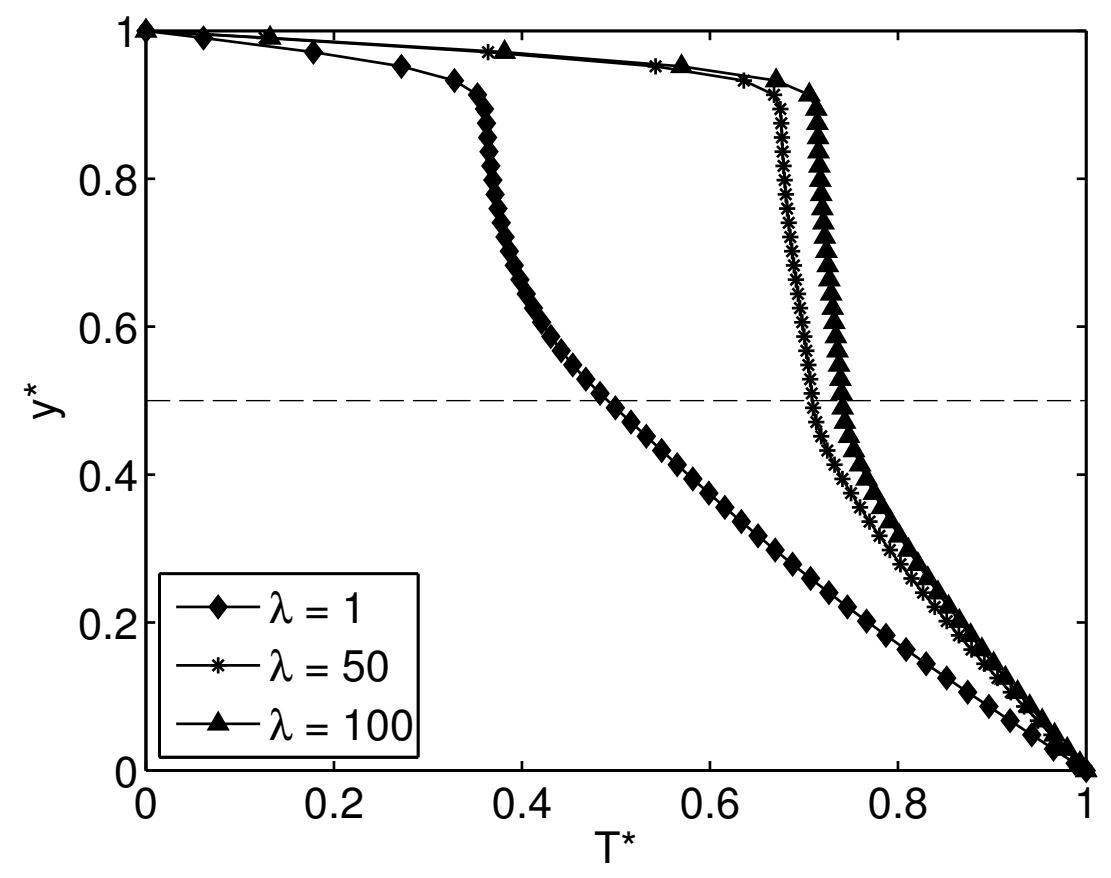

Figure 4.33: Dimensionless temperature $\left(T^{*}=T-T_{C} / T_{H}-T_{C}\right)$ profiles along the vertical centerline of the domain for different conductivity ratios. $\delta=0.5, \eta=0.5$ and $R a=5 \times 10^{5}$.

Finally, the temperature profiles along the centerline of the cavity at a Rayleigh number of $5 \times 10^{5}$ are shown for the three conductivity ratios. As can be expected, the profiles for the higher and lower conductivity ratios are widely different. For small values of $\lambda$, there is rapid drop in temperature across the porous layer after which the temperature drop in the fluid layer is very low except near the upper surface. However, for higher values of $\lambda$, there is a very small drop in temperature across the porous layer and practically no drop in the temperature in the fluid layer. Almost all the temperature change occurs near the upper surface where there exists a very large temperature gradient. This was seen earlier from the isotherm patterns. This shows that heat transfer across the cavity takes place differently for high and low conductivity ratios. For low values of $\lambda(1 \leq \lambda \leq 10)$, the bulk of the heat influx into the system is rapidly channeled away near the heater surface. On the other hand, for larger values of $\lambda$, the bulk of the heater influx is transferred to the cold surface very near the upper wall.

\subsubsection{Effect of the Prandtl number}

The effect of the Prandtl number has not been covered in prior studies of convection in fluid-superposed porous layers heated from below and hence there is no benchmark to compare the present results against. As such, wherever possible, results will be compared with prior studies on convection in fluid and porous layers. Two different values of the 
(a)
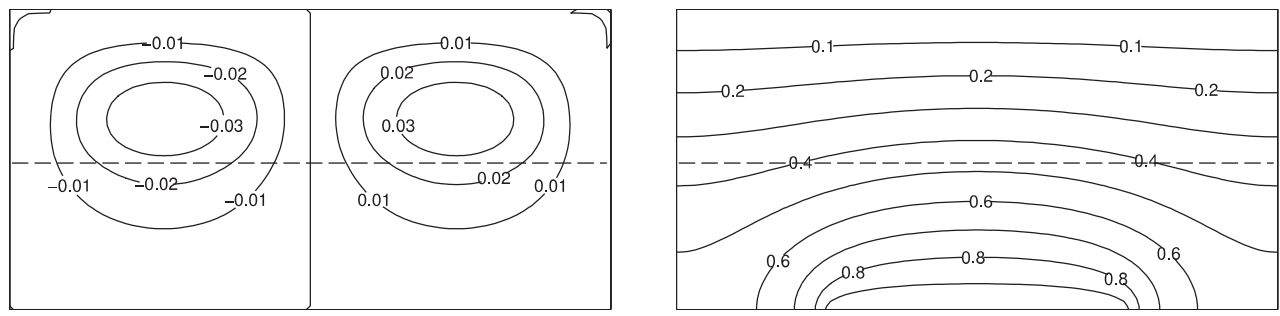

(b)
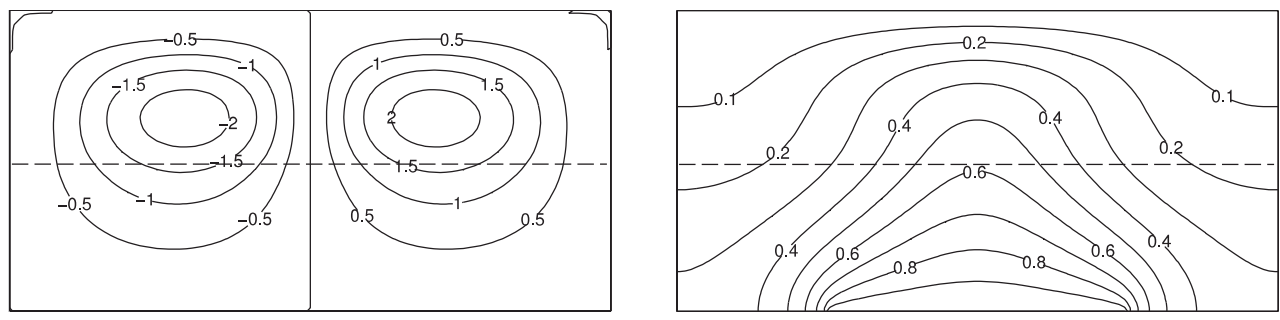

(c)
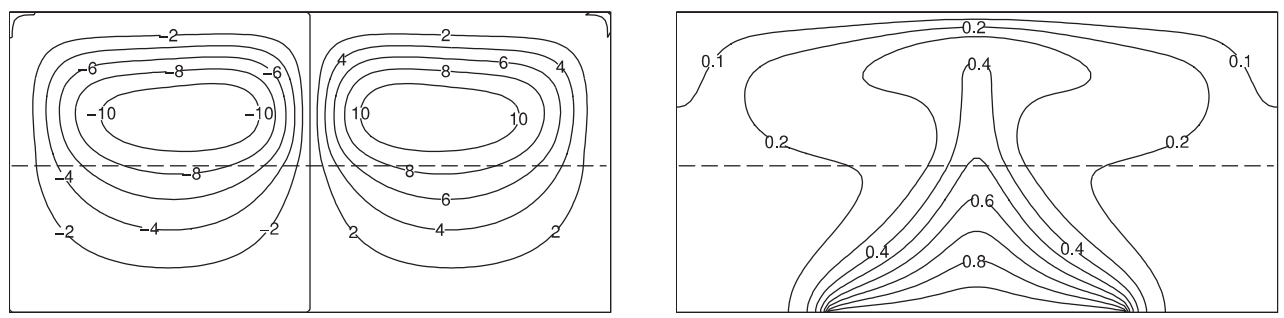

(d)
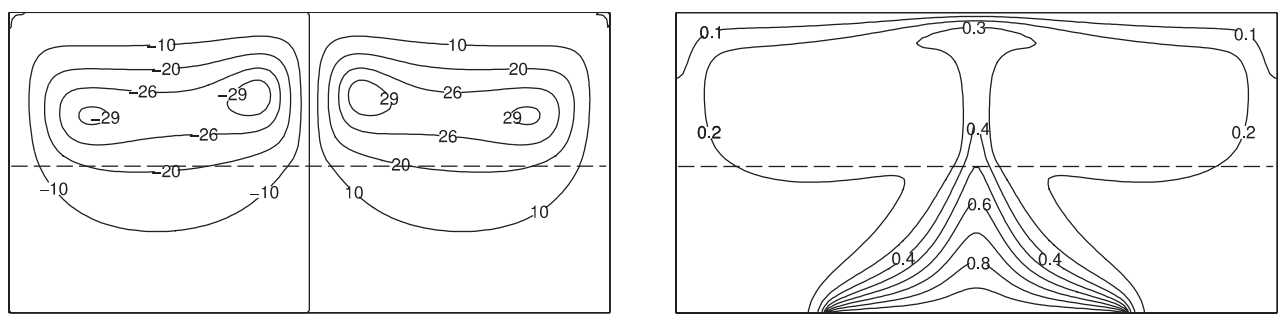

Figure 4.34: Streamline and isotherm patterns for convection in a composite layer for $\operatorname{Pr}=$ $7, \delta=0.5$. (a) $R a=10^{3}$, (b) $R a=10^{4}$, (c) $R a=10^{5}$, and (d) $R a=10^{6}$.

Prandtl number are considered here: $\operatorname{Pr}=0.7$ and 7 . These are approximately equal to the Prandtl numbers of air $\left(\operatorname{Pr}=0.71\right.$ at $\left.25{ }^{\circ} \mathrm{C}\right)$ and water $\left(\operatorname{Pr}=6.26\right.$ at $\left.25{ }^{\circ} \mathrm{C}\right)$ respectively. These fluids have been selected for two primary reasons. First, becasue these two fluids are most commonly encountered in studies on fluid and porous media convection, there are several references available in the literature for comparison. Also, for these fluids, there is not a significant change in the fluid viscosity, and hence the Prandtl number, with temperature. For fluids with higher viscosity, the temperature dependence of Prandtl number cannot be ignored. However, because the present model is valid only for constant $\operatorname{Pr}$, high viscosity fluids, though very important in a large number of applications, are not considered in the present study.

The flow and temperature fields for $\operatorname{Pr}=0.7$ and 7 are shown in Figures 4.34 
(a)

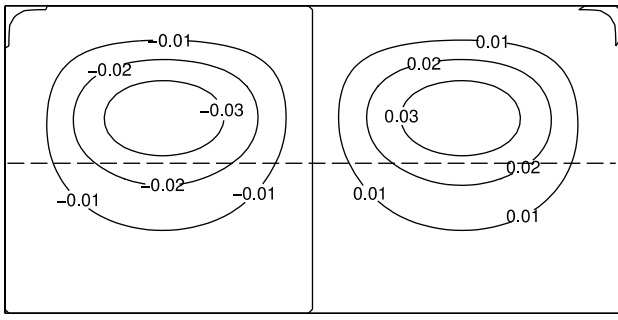

(b)

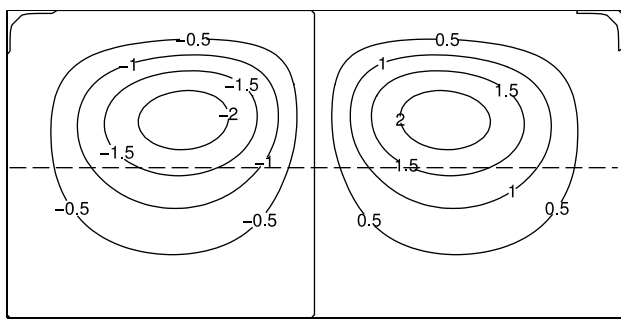

(c)

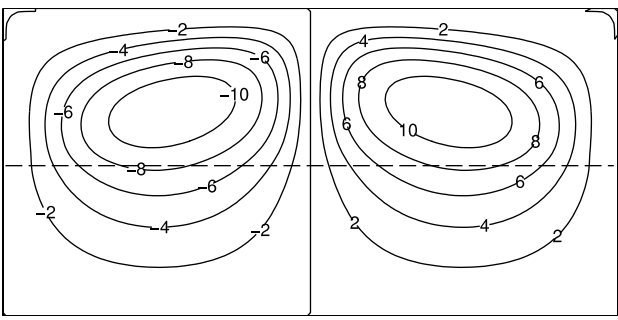

(d)

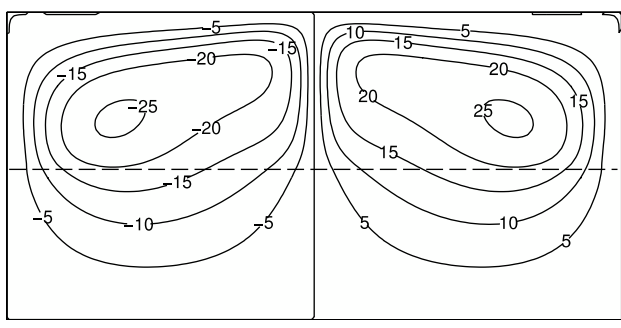

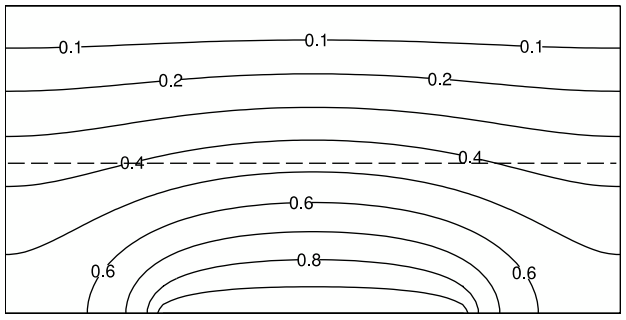
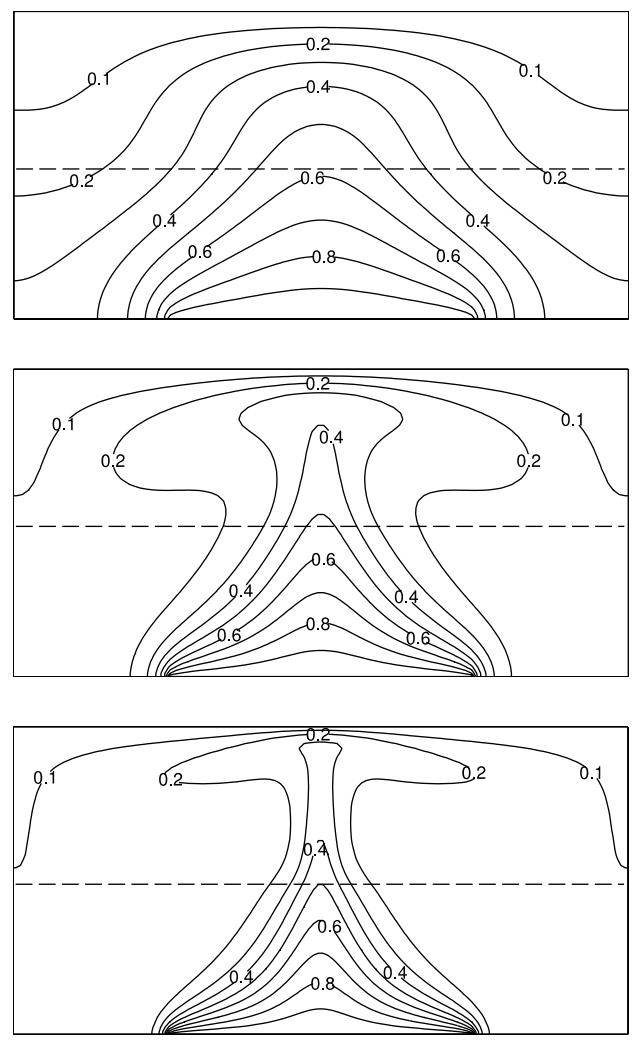

Figure 4.35: Streamline and isotherm patterns for convection in a composite layer for $\operatorname{Pr}=$ $0.7, \delta=0.5$. (a) $R a=10^{3}$, (b) $R a=10^{4}$, (c) $R a=10^{5}$, and (d) $R a=10^{6}$.

and 4.35, respectively. At $R a=10^{3}$ the flow patterns for both fluids look identical. Two circulating cells produced due to end effects at the heater are seen and the fluid velocities are identical. Similarly, the isotherms indicate that the heat transfer is conduction dominated. With increase in the Rayleigh number to $10^{4}$, much of same flow patterns seen earlier in the chapter - initiation of convection dominated heat transfer, strengthening of the existing cellular flow patterns, and confinement of the flow to the upper fluid layer - are seen for both fluids. Fluid velocities too are almost the same in both cases as seen from the value of the stream function. Similarly the isotherms show a gradually developing thermal plume as convection heat transfer rates start increasing.

When the Rayleigh number is increased further to $10^{5}$, however, different streamline 
patterns are seen for the two fluids. For $\operatorname{Pr}=0.7$, the fluid velocities within the core of the circulation cells are at an angle to the fluid-porous layer interface. On the other hand, for $\operatorname{Pr}=7$, flow velocities within the core of the cellular flow patterns are parallel to the interface. This effect occurs due to lower shear resistance in the low Prandtl number fluid and is also seen in simulations of Rayleigh-Bénard problem with fluids of different velocities. However, the maximum absolute values of the stream functions are almost identical in both cases. This indicates that the fluid velocities, though having different directions within the core, have approximately the same velocities. The isotherm patterns however look virtually identical indicating that heat transfer rates for the two fluids are almost same. Finally, when the Rayleigh number is increased to $10^{6}$, the streamline patterns for $\operatorname{Pr}=0.7$ become highly skewed and unsymmetrical. The core of the convection cells is seen to become narrow near the rising plume, i.e., near the vertical centerline of the cavity, while the other end is noticeably larger and has a small pocket of higher velocities. For $\operatorname{Pr}=7$, however, the convective cells are symmetrical and show two small pockets of higher fluid velocities. The isotherms show a narrow rising plume while the region outside the plume is largely isothermal, especially in the porous layer.

The Nusselt-versus Rayleigh number relationships for different Prandtl numbers is shown in Figure 4.36. As can be seen, the two curves are almost identical indicating that the Prandtl number has little effect on the overall heat transfer rates of the system. This observation is in accordance with the results of both the Rayleigh-Bénard problem and the Horton-Rogers-Lapwood problem. The only noticeable difference in the overall heat transfer coefficients can be seen for $R a>10^{5}$ and can be directly attributed to the difference in the flow patterns at $R a=10^{6}$. The centerline temperature profiles for $R a=10^{5}$ do not show much difference between the two systems. Based on all the above arguments it can be concluded that the Prandtl number is not a significant parameter in determining the overall heat transfer characteristics of the present system.

\subsection{Evolution of the flow and temperature fields}

To get a fundamental understanding of the mechanism by which the convective flow develops from an initial disturbance, two cases are chosen: convection in a fluid-superposed porous layer with a uniformly heated base and convection in a similar system with a localized heating at the base. For these two simulations, the following parameters are kept fixed: $R a=10^{5}, \eta=0.5, D a=10^{-6}, A=2, \lambda=1$, and $\operatorname{Pr}=7$. Only the heater-to-base length ratio, $\delta$, is different for the two cases. In the first case $\delta=1$ and the second case $\delta=0.5$.

The evolution of the flow and temperature fields for the two cases is shown in Figures 4.38 and 4.39. The figures essentially show snapshots of the streamlines and isotherms at 


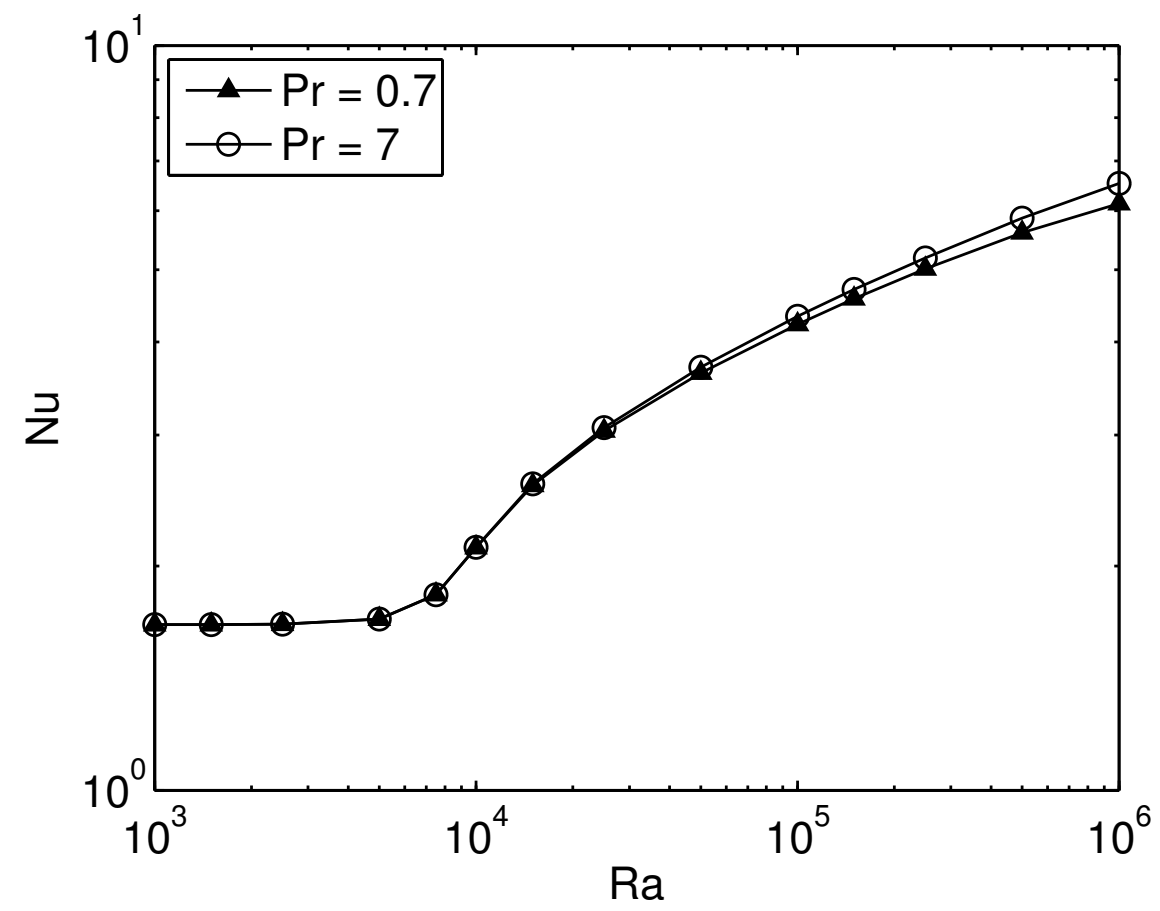

Figure 4.36: Nusselt-versus-Rayleigh number relationship for different Prandtl numbers. $\delta$ $=0.5$ and $\eta=0.5$.

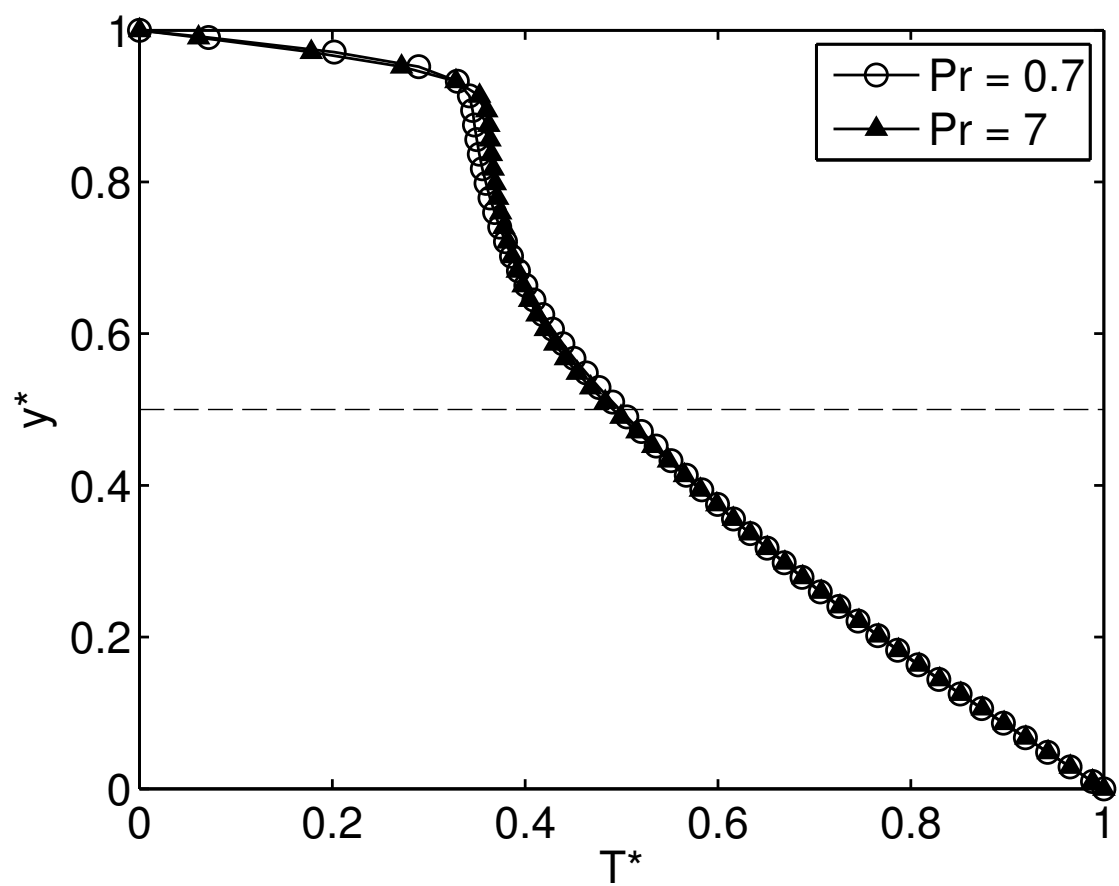

Figure 4.37: Dimensionless temperature $\left(T^{*}=T-T_{C} / T_{H}-T_{C}\right)$ profiles along the vertical centerline of the domain for different Prandtl numbers. $\delta=0.5, \eta=0.5$ and $R a=5 \times 10^{5}$. 
various values of the dimensionless time $t^{*}$ starting from $t^{*}=0$ to the final steady-state. For $\delta=1$, the solution is initiated by introducing a small sinusoidal perturbation to the temperature field at $t^{*}=0$. This is shown in 4.38(a). At $t^{*}=1$, two convective cells are seen. The flow at this time is almost entirely restricted to the overlying fluid layer. The corresponding isotherms corroborate this fact: the temperature field inside the porous layer resembles a conduction heat transfer mode and some convection can be seen in the fluid layer. At $t^{*}=40$, a much more well defined flow pattern emerges. Circulatory fluid motion in present in the entire fluid layer and signs of flow penetration into the porous layer appear. The corresponding isotherm patterns show a plume like flow in an early stage of development. When $t^{*}=60$, an increase in the velocities of convective motion accompanied by further flow penetration in to the porous layer occurs. Simultaneously, the thermal plume becomes better defined. As time progresses this process continues until steady state is attained. The flow patterns do not show any signs of oscillatory motion. This is consistent with earlier findings on convection in fluid-superposed porous layers heated uniformly $[36,39]$.

When $\delta=0.5$, the flow evolution progresses in a slightly different manner. In this case no initial perturbation is given to initiate the iterative solution. Rather the horizontal temperature gradient at the edge of the heater triggers the onset of convective motion. This can be seen in Figure 4.39(a) where two small end cells are seen at the edge of the heater. Once the onset of convective motion is triggered a weak circulatory fluid flow is seen in the upper fluid layer at $t^{*}=10$. The corresponding isotherms, however, show only a small disturbance to the conduction temperature field indicating that convective motion has not yet commenced. At $t^{*}=50$, the first signs of the onset of convective motion can be seen. A non-negligible circulatory flow pattern develops in the fluid layer and rising thermal plume begins to take shape. Thereafter, the flow evolution essentially follows the same path towards steady state as $\delta=1$ : flow intensities keep increasing; there is a flow penetration in to the underlying porous layer, and a narrow thermal plume rises from the center of the heater. Thus the presence of a localized heating source does not affect the steady-state convection pattern or the path to steady-state; it merely provides different trigger for the onset of convective motion.

\subsection{Conclusions}

From the numerical results presented in this chapter, the following conclusions can be drawn:

- The presence of a localized heat source does not affect the mode of convective motion but merely provides a different trigger for the onset of convection. Also overall heat 
(a)

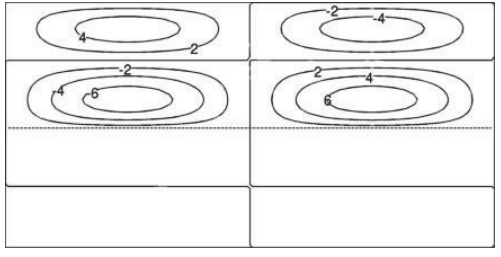

(b)

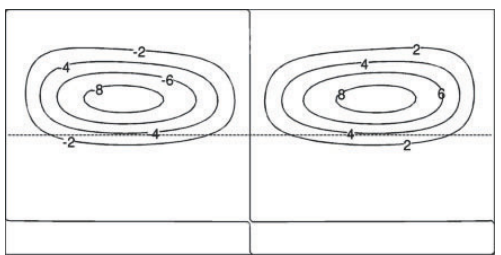

(c)

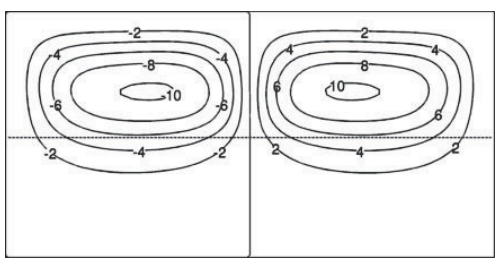

(d)

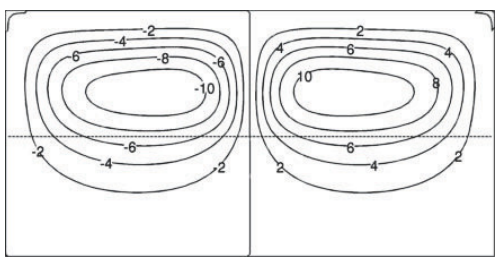

(e)

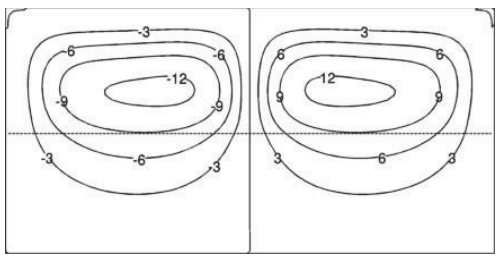

(f)

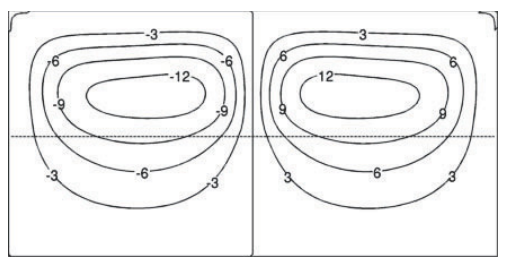

(g)

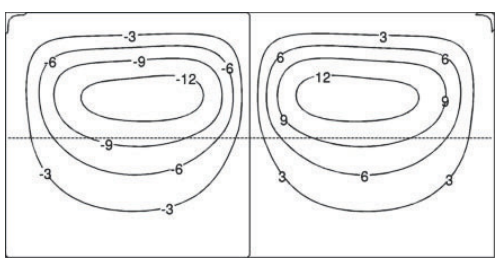

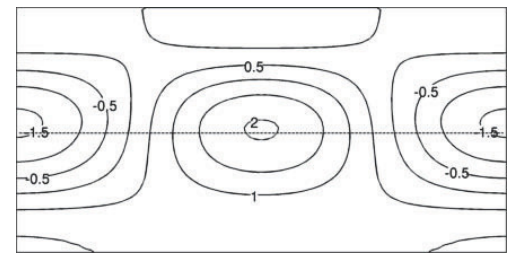
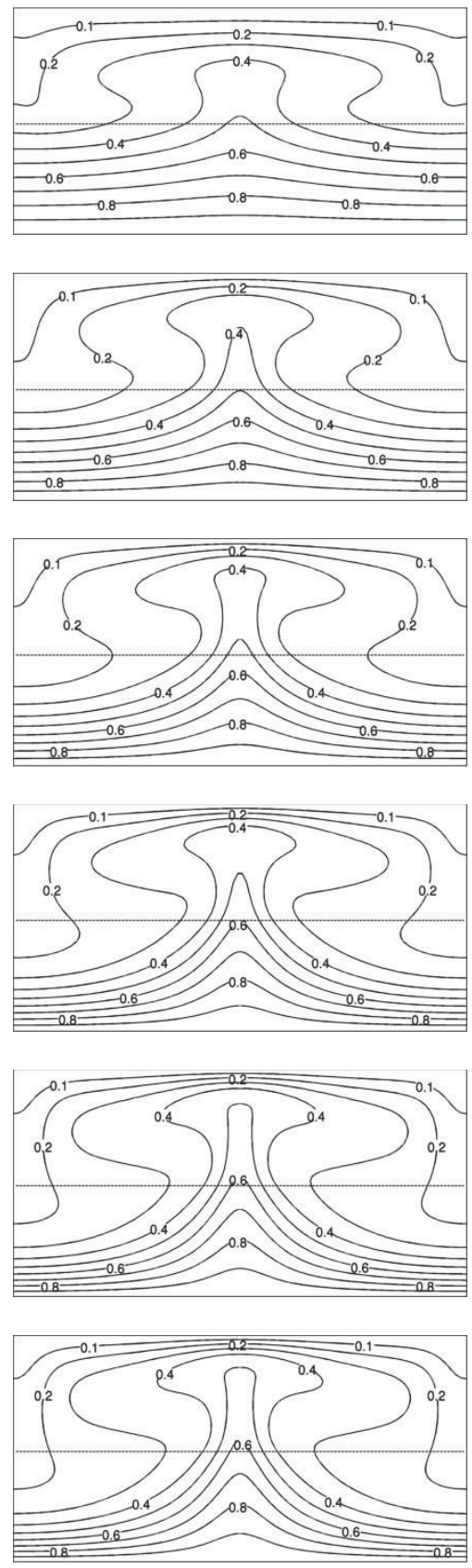

Figure 4.38: Evolution of the flow and temperature fields in fluid-superposed porous layers heated uniformly from below. (a) $t^{*}=0$, (b) $t^{*}=1$, (c) $t^{*}=5$, (d) $t^{*}=10$, (e) $t^{*}=20$, (f) $t^{*}=40$, and (g) $t^{*}=60 . R a=10^{5}, \delta=1, \eta=0.5, \operatorname{Pr}=$ $7, D a=10-6, A=2, \lambda=1$. 
(a)

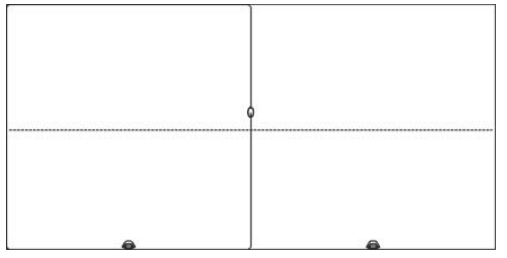

(b)

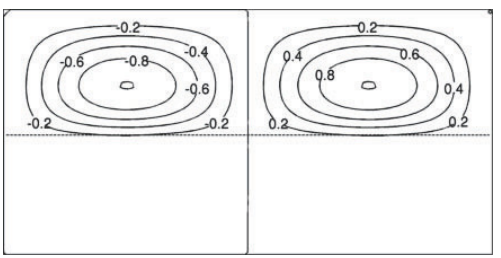

(c)

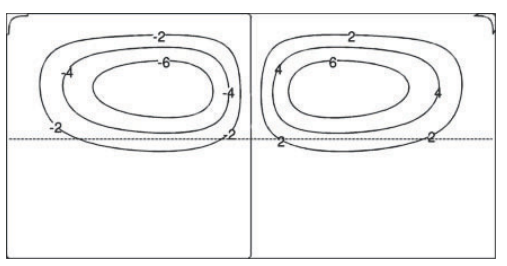

(d)

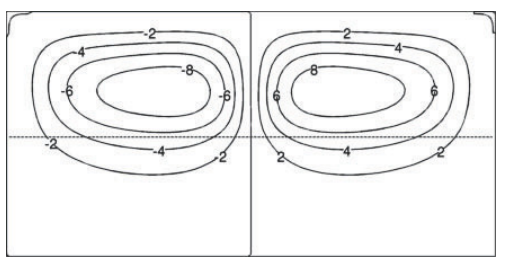

(e)

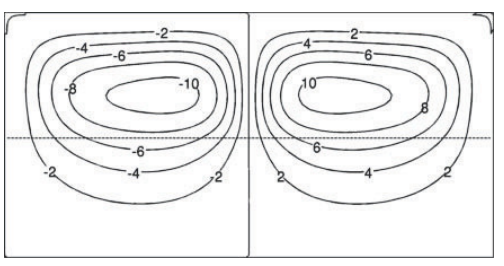

(f)

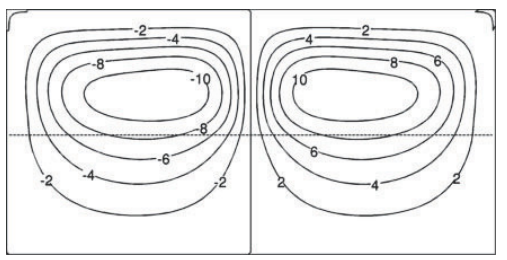

(g)

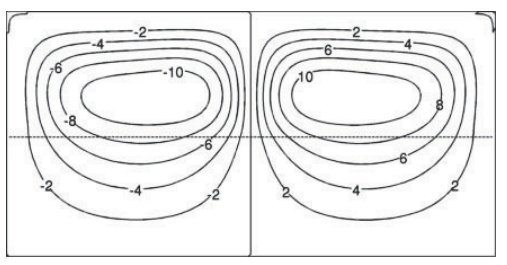

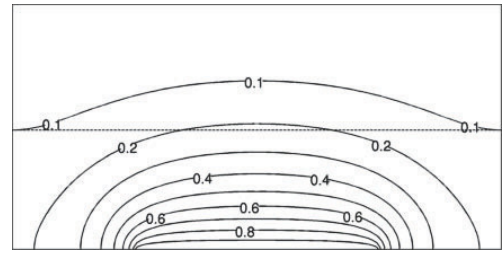
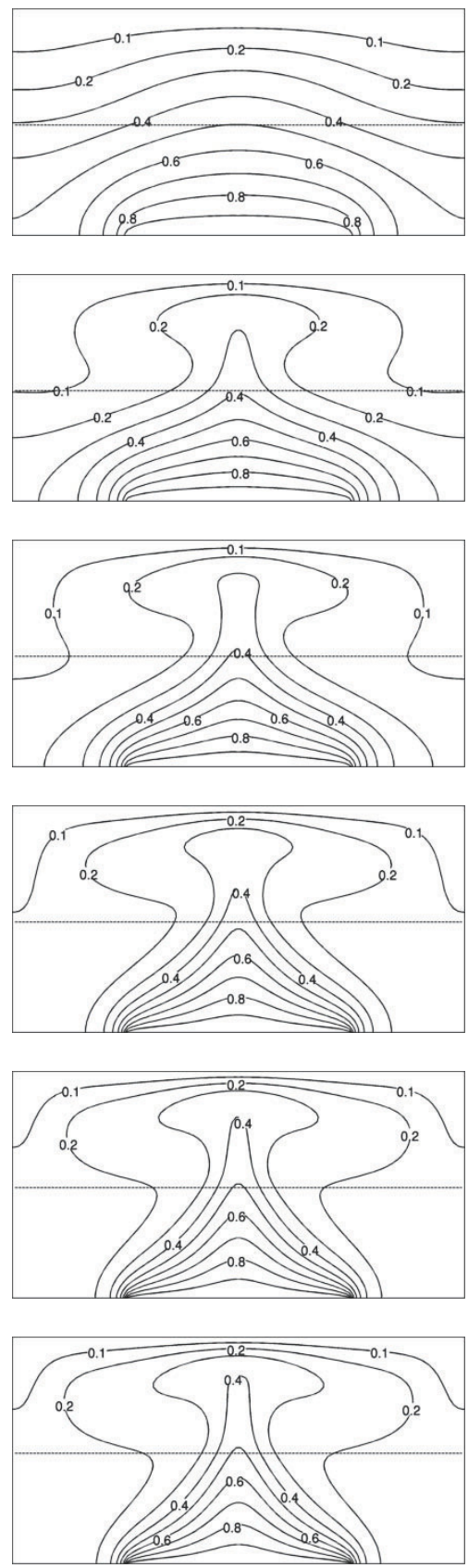

Figure 4.39: Evolution of the flow and temperature fields in fluid-superposed porous layers heated uniformly from below. (a) $t^{*}=0$, (b) $t^{*}=1$, (c) $t^{*}=5$, (d) $t^{*}=10$, (e) $t^{*}=20$, (f) $t^{*}=40$, and (g) $t^{*}=60 . R a=10^{5}, \delta=0.5, \eta=0.5, \operatorname{Pr}=$ $7, D a=10-6, A=2, \lambda=1$. 
transfer rates increase with decrease in the heater length fraction.

- Fluid motion is primarily confined to the upper fluid layer with penetrative convective motion in to the lower fluid layer.

- Overall heat transfer rates increase with increase in the height of the fluid layer. A similar trend is noticed with an increase in the Darcy number. Both of these effects can be attributed to the same fundamental cause: a decrease in the resistance to fluid motion offered by the porous layer.

- The effect of the aspect ratio on the flow structure and overall heat transfer rates is complex and depends also on the heater length and the Rayleigh number. For a given heater length fraction, heat transfer rates decrease slightly with an increase in the aspect ratio. Also the number of convective cells at a given aspect ratio and heater length fraction is found to change with increasing Rayleigh number.

- Increase in the solid-to-fluid conductivity ratio leads to significant enhancements in the overall heat transfer rates. This is not only due to higher conductivity of the solid matrix but also due to an increase in the intensity of the convective motion with increase in the conductivity ratio.

- The Prandtl number has very little effect on the overall heat transfer rates except at very high Rayleigh numbers. 


\section{Chapter 5}

\section{Experimental Design and Procedure}

In this chapter, the design, setup and procedure for the experiments is discussed in detail. Because of the large number of independent controlling parameters, a complete parametric experimental investigation of the present problem is a challenging task. As such, an exhaustive experimental study is not undertaken in the present study. Instead, a limited set of experiments is performed to attempt a validation the numerical solutions. To that end, this chapter begins with a discussion on the design of the experiments. Thereafter, a detailed description of the experimental apparatus and the associated instrumentation is given. Finally, the experimental procedure adopted is discussed briefly.

\subsection{Design of experiments}

The first step in the experimental study is to carefully design the experiments that need to be performed. As mentioned above, the principal aim of the experimental study is to validate the predictions of the numerical simulations. In addition, it is also desirable that the experiments be designed in a manner such that overall heat transfer coefficients can be measured for high Rayleigh numbers, a parametric range that cannot be explored by numerical simulations. In this way, a combination of numerical simulations and experimental measurements can be used to explore natural convection in fluid-superposed porous layers over a wide range of Rayleigh numbers. In order to achieve this with the minimum number of experiments, a statistical design of experiments is necessary. To perform a systematic design of experiments, it is essential to first clearly identify the quantities that need to be measured. The main aim of the experiments is to express the overall heat transfer rates for different experimental conditions in terms of the overall Nusselt and Rayleigh numbers, 


$$
\begin{gathered}
N u=\frac{q^{\prime \prime} H}{k_{\mathrm{f}}\left(T_{H, \mathrm{avg}}-T_{C, \mathrm{avg}}\right)}, \\
R a=\frac{g \beta H^{3}\left(T_{H, \mathrm{avg}}-T_{C, \mathrm{avg}}\right)}{\nu_{\mathrm{f}} \alpha_{\mathrm{f}}} .
\end{gathered}
$$

Here $q^{\prime \prime}$ is the net heat flux supplied to the system, $T_{H, \text { avg }}$ and $T_{C \text {,avg }}$ are the average temperatures along the heater and top surfaces, respectively, and the other quantities have their usual meanings. The quantities that need to be measured during each experiment are the average cooled and heated surface temperatures, and the net heat flux supplied by the heaters.

The next step in the design process is to identify the most important controlling parameters for the present problem. As seen in the previous chapter, there are six primary independent parameters for the problem, namely, the heater-to-base length ratio, $\delta$, the porous-to-overall height ratio, $\eta$, the aspect ratio, $A$, the Darcy number, $D a$, the solid-tofluid conductivity ratio, $\lambda$, and the fluid Prandtl number, Pr. In addition, the following parameters, which were assumed to be constant for the numerical simulations, must also be considered: the porosity of the porous layer, $\phi$, the Forchheimer number, $F$, and the specific heat ratio, $\sigma$. There are thus a total of nine independent controlling parameters for the present problem. Even at the minimum value of two levels for each parameter, the total number of experiments is $2^{9}=512$. Because it is practically impossible to perform such a large number of experiments, it is essential to identify those parameters which primarily govern the heat transfer mechanism in the system. For that, the following considerations are made:

- Measurements will be made only for steady-state convection, and therefore the specific heat ratio, does not need to be considered.

- For random stacking of spherical particles, the porosity $\phi$ is approximately in the range of $0.36-0.4$ and can hence be considered to be constant.

- For constant porosity, the Darcy and the Forchheimer numbers can be considered to be functions of the non-dimensional bead diameter, $\gamma$.

- Based on the numerical simulations, it can be concluded that the fluid Prandtl number and the aspect ratio of the system do not have a significant effect on the overall heat transfer rates, and hence their effect can be neglected 
Based on the above considerations, the primary controlling parameters for the present problem are the heater length ratio, $\delta$, the height ratio, $\eta$, the conductivity ratio, $\lambda$, and the non-dimensional bead diameter, $\gamma$. If, however, only a single set of beads is used for all the experiments, both $\lambda$ and $\gamma$ will be constant and the effects of only two independent parameters will need to be investigated. This will drastically reduce the total number of experiments to be performed. In addition, from the numerical simulations, it is clear that the two most important controlling parameters for the present problem are the height ratio, $\eta$, and the heater length ratio, $\delta$. Therefore selecting a single set of beads for the porous layer will enable a very careful understanding of the effect of these two parameters. In the present experiments $3 \mathrm{~mm}$ DIA sodasilicate glass beads are chosen for the porous layer, with water as the saturating fluid. The physical and thermal properties of the beads are given in Appendix B. With this, the aim of the present set of experiments can be summarized as: i) understanding the effect of $\eta$ at a particular value of $\delta$, and ii) understanding the effect of $\delta$ at a particular value of $\eta$.

In order to meet these, the number of levels to be studied for each parameter, as well the values of the parameters at each of those levels needs to be delimited. From a statistical perspective, the minimum number of levels for each parameter is two. A higher number of levels for each parameter can lead to a better understanding of its effect on the overall heat transfer rates but may not be experimentally feasible. Keeping in mind the fact that the overall objective of the experimental study is to validate the predictions of the numerical simulations, the following design is adopted for the experiments: i) heater length ratio: two levels, and ii) height ratio: four levels.

It must be noted that in selecting the number of levels for each parameter, primary consideration has been given to the practical feasibility of conducting different sets of experiments. Conducting experiments with different heater length ratios is a challenging task as it requires significant changes to be made to the apparatus before experiments with a different heater size can be performed. The main and guard heaters, as well as the thermocouples for measuring the heater temperatures must be removed and a new set of heaters along with the associated instrumentation must be re-installed. Because of this, only two levels are selected for studying the effects of $\delta$. Experiments with different porous layer height ratios however do not need any changes to be made to the apparatus. Thus, four levels of $\eta$ are chosen. Of these, one level $(\eta=1)$ is selected for investigation to validate the experimental setup with literature data on convection in porous layers heated locally from below.

In order to select the values of $\delta$ and $\eta$ for the different levels, several other factors, such as the availability of heater sizes, the bead size, the working height, and the range of Rayleigh numbers desired need to be considered. Selection of the two values of $\delta$ is fairly 
Table 5.1: Overview of the experimental design selected.

\begin{tabular}{|l|c|c|c|c|c|}
\hline Experiment & $\delta$ & $\eta$ & $\begin{array}{c}\text { Porous layer } \\
\text { height }(\mathrm{cm})\end{array}$ & $\Delta T\left({ }^{\circ} \mathrm{C}\right)$ & $R a$ \\
\hline \hline $\begin{array}{l}\text { 1. Validation } \\
\text { of setup }\end{array}$ & 0.44 & 1 & 3.81 & $3-40$ & $3 \times 10^{6}-9 \times 10^{7}$ \\
\hline 2. Effect of $\eta$ & 0.44 & 0.67 & $\begin{array}{l}2.54 \\
2.86\end{array}$ & $3-40$ & $3 \times 10^{6}-9 \times 10^{7}$ \\
\hline 3. Effect of $\delta$ & 0.11 & 0.5 & 1.91 & $3-40$ & $3 \times 10^{6}-9 \times 10^{7}$ \\
\hline
\end{tabular}

straightforward. For the required heater lengths, two different heater widths ( $W$ in Figure $5.2)$ are available: $2.54 \mathrm{~cm}$ and $10.16 \mathrm{~cm}$, for which the two values of $\delta$ are 0.11 and 0.44 respectively. Selection of the appropriate values of $\eta$ requires consideration of the desired range of Rayleigh numbers. Because the upper end of $R a$ for the simulations is in the range $10^{6}-5 \times 10^{6}$, it is desirable to have the lower end of the experimental data in this Rayleigh number range. Recalling the definition of the overall Rayleigh number and noting that the minimum temperature difference that can be maintained across the heater surface and the top plate is $\sim 2-5{ }^{\circ} \mathrm{C}$, a working height of $3.81 \mathrm{~cm}(1.5 \mathrm{in}$.) would give the required lower $R a$ range.

In order to select the different height ratios, consideration must also be given to the effect of the dimensionless bead diameter, $\gamma$. It is well known that the porosity of porous layers keeps increasing as $\gamma$ approaches unity. This is because the pore space adjacent to any surface is about $20 \%$ higher than in the interior of the porous layer $[1,2]$. Katto and Masuoka [30] have shown that end effects can be neglected when $\gamma \leq 0.2$, i.e., when the porous layer is at least five particle diameters. In the present study, the porous layer is selected to be at least six particle diameters high or in other words, $\left(H_{\mathrm{m}}\right)_{\min }=1.8 \mathrm{~cm}$. With this consideration, the different experimental values of $\eta$ can be selected. A detailed design of experiments is shown in Table 5.1.

\subsection{Design of experimental setup}

The design of the experimental setup is the most critical part of the experimental study as the accuracy of the apparatus design directly affects the accuracy of the obtained results. The primary challenge in designing the experimental system is in ensuring that the final setup is an accurate physical representation of the problem under investigation. In addition, the setup must be designed in a way such that all quantities of interest can be 
accurately measured with minimal uncertainty. The primary design challenges are:

1. Minimizing uncertainties: Boundary conditions must be maintained accurately so that the set up conforms to the problem definition. This implies that adiabatic surfaces must be carefully insulated, the test chamber must be adequately sealed to prevent fluid leakage, and suitable guard heaters must installed to prevent heat loss from the heater and ensure a constant heat flux boundary condition.

2. Material selection: Both thermal and mechanical consideration must be included in the selection of materials for fabricating the various components of the set up. These considerations include thermal conductivity, mechanical strength, and machinability.

3. Accurate instrumentation: Instrumentation involved in measuring the various quantities of interest is an integral part of the experimental apparatus. The incorporation of instrumentation, like heater leads and thermocouple wires, into the experimental apparatus can introduce several design challenges which must be given due consideration.

4. Operator ease: The experimental apparatus needs to be designed for maximum experimental flexibility. Therefore, a modular design approach is desirable so that different sets of experiments can be performed by making minimal changes to the apparatus.

Each of the above mentioned factors must be carefully considered while designing the experimental setup. In order to simplify the design process, it is easier to break up the experimental setup into two distinct components: a) the experimental apparatus, and b) the instrumentation and final assembly. Each of these components can be individually fabricated and assembled for the final setup and hence it is easier to consider them one by one.

\subsubsection{Experimental apparatus}

The experimental apparatus is the primary component of the overall experimental setup. The apparatus consists of a test chamber that holds the fluid-superposed porous layer and provides the required heated and cooled surfaces at the bottom and at the top. The guiding principle behind the design of the apparatus is that it must be easily assembled and disassembled. Because of this requirement, a modular design approach is necessary. With this approach the apparatus is built by assembling several independent modules, each of which is independently fabricated and assembled. This ensures that the apparatus can be easily disassembled and then reassembled after making the required modifications for a particular experiment. This approach also helps in incorporating instrumentation into 
the apparatus during the setup of the entire experimental system. The apparatus used in the present experiments is made up of three modules: the convection cell, the base plate assembly, and the top plate assembly. Figure 5.1 shows a schematic diagram of a breakdown of the apparatus into the different modules.

\subsubsection{Convection cell}

The convection cell is the fundamental component of the experimental setup. It provides a test chamber which holds the porous and fluid layers and in which the experiments are conducted. In order to design the cell, close attention must be paid to the following factors: i) it must be long enough to ensure that end-effects do not have a significant influence on the heat transfer characteristics of the system; ii) it must have sufficient width in order to allow three dimensional effects to be manifested; iii) the total height of the cell must not be too great as with increasing height, the temperature difference between the top and bottom surfaces needed to induce the onset of convection increases which can lead to appreciable variations in the thermophysical properties of the fluid; iv) The convection cell must be constructed out of a material that has low thermal conductivity in order to minimize heat losses through the walls.

The material must also be easily machinable to facilitate easy construction. Based on the above factors, a $22.86 \mathrm{~cm}$ long, $22.86 \mathrm{~cm}$ wide and $25.4 \mathrm{~cm}$ high convection cell is chosen for the experiments. This is shown schematically in Figure 5.2. The walls of the cell are constructed out of $1.27 \mathrm{~cm}$ thick polycarbonate, which is chosen for its low thermal conductivity and ease of machining. It must be noted that the height of the convection cell is much larger than what is required for the present experiments. The height of the cell has been deliberately kept large keeping in mind further extensions of the present work where a large working height might be required. The required working height is obtained by making the height of the top plate adjustable. This design approach allows the selection of any working height or aspect ratio depending on the requirements of a particular experiment and provides the necessary flexibility for conducting future experiments.

\subsubsection{Base plate assembly}

The base plate assembly comprises of the base plate, the main heaters, and the guard heaters. Figure 5.3 shows a schematic diagram of the base plate assembly. As can be seen, the base plate forms the principal part of the assembly. The main heater and the guard heater are attached to the upper and lower sides of the base plate respectively. The primary requirement of the base plate is to maintain the uniform heat flux boundary condition at the heated section of the base, i.e., preventing heat loss through the base so that the flux produced by the heaters is supplied entirely to the porous layer above. From a one-dimensional steady state heat conduction model, the heat loss through the base is, 


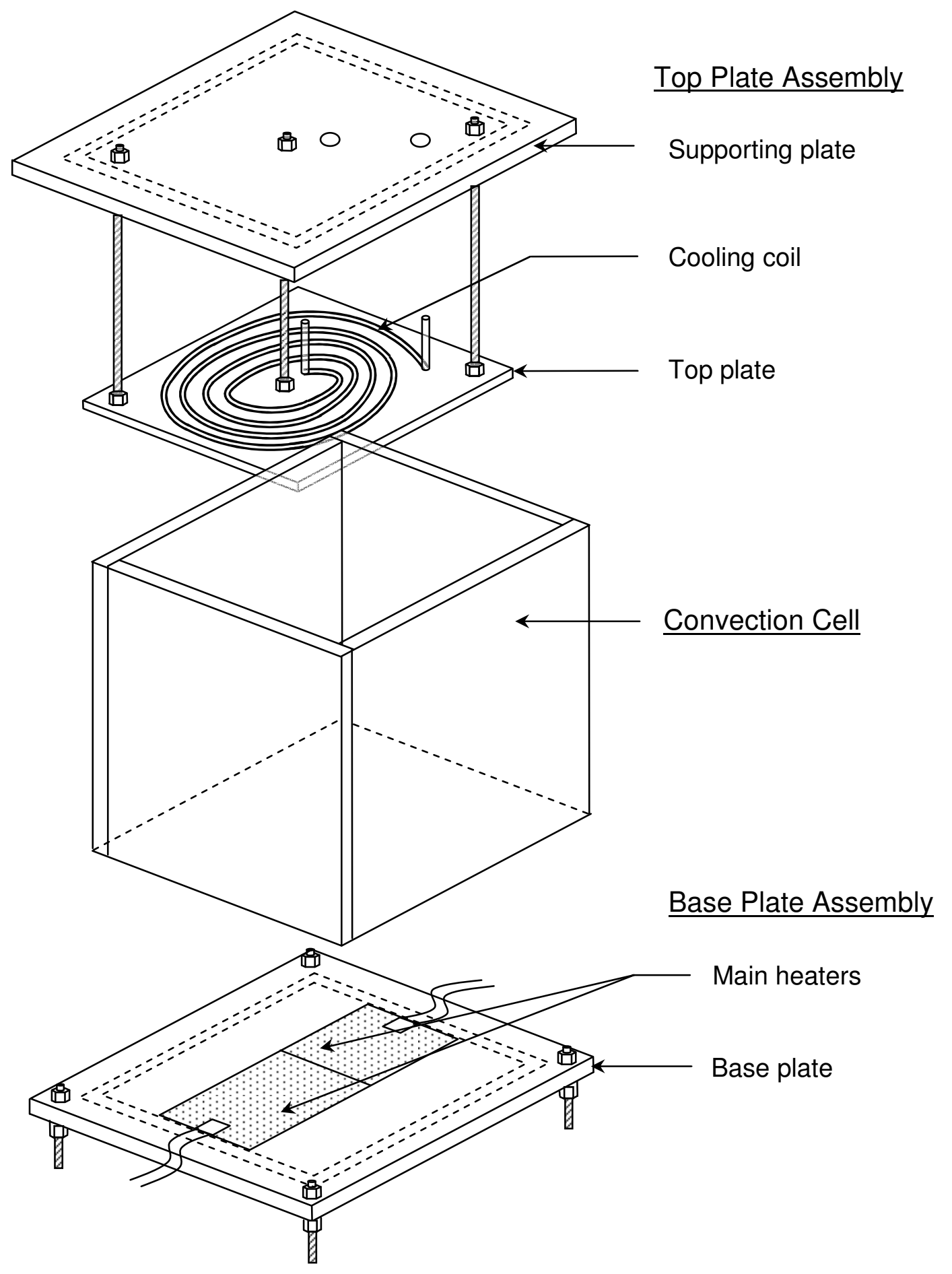

Figure 5.1: Schematic of experimental setup showing the three different modules. 
(a)

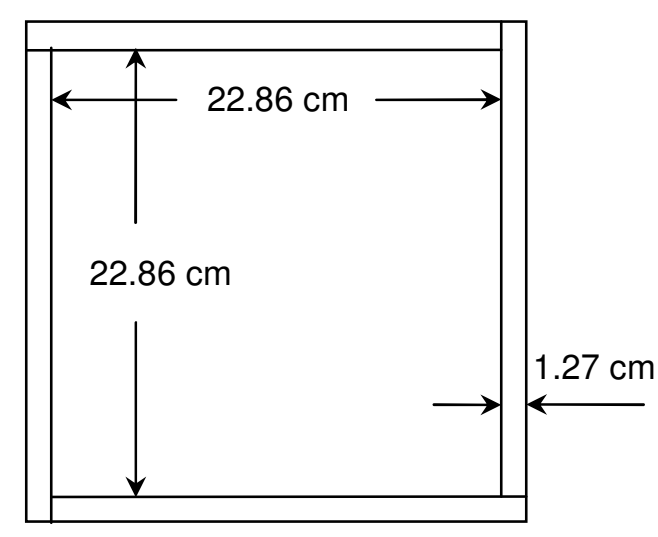

(b)

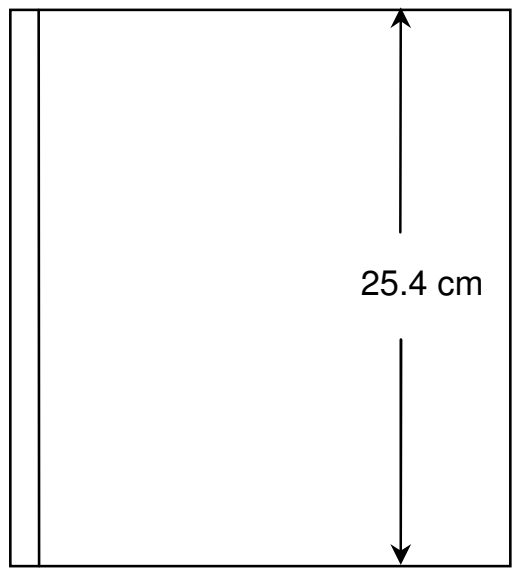

Figure 5.2: Schematic of the convection cell showing (a) the top view and, (b) the front view.

$$
q_{\text {loss }}^{\prime \prime}=-k_{\text {base }} \frac{\Delta T_{\text {base }}}{H_{\text {base }}}
$$

where $q_{\text {loss }}^{\prime \prime}, k_{\text {base }}, \Delta T_{\text {base }}$ and $H_{\text {base }}$ are the heat flux through the base, the thermal conductivity of the base plate, the temperature difference across the plate and the thickness of the plate, respectively.

From equation (5.3), it is clear the heat loss through the base can be minimized by reducing the temperature difference across the plate, increasing the thickness of the plate, or reducing the plate thermal conductivity. A large plate thickness, however, would make assembly of the apparatus very cumbersome. A better alternative is to fabricate the base plate from a material which has a low thermal conductivity. The thermal and physical properties of polycarbonate are listed in Appendix B. For the present experiments, the base plate is constructed out of $1.27 \mathrm{~cm}$ thick polycarbonate sheet. No material however is perfectly insulating and a finite amount of heat loss always takes place for any temperature gradient across the plate. By employing a guard heater, however, this heat loss can be compensated. This is explained later.

The main heater is attached to the top of the base. The primary purpose of the main heater is to provide a constant heat flux into the overlying composite fluid-porous layer over a wide range of temperatures. Flexible wire-wound silicon rubber heaters (Watlow, St. Louis, MO) are used in the present experiments as the main heaters. These heaters are chosen because of their ruggedness, reliability, and ease of handling. The heaters are of a sandwich-type construction and consist of a heating element sandwiched between two 
sheets of silicon rubber. The heating element is constructed by winding fine resistance wires around a fiberglass cord. The primary advantage of these heaters is their ability to provide a highly uniform heat flux and maintain a constant resistance over a wide range of temperatures. Another advantage of these heaters is that they can be easily attached to and removed from the base plate. The heaters are provided with a pressure sensitive adhesive on their lower surface and can be attached to the base plate by placing them in position and applying pressure. After use for a particular experiment, they can be removed by simply peeling them off the plate, much like adhesive tape. This ease of handling provides great flexibility in experimental design as it allows heaters of different sizes to be used for different sets of experiments. In the present experiments, the main heater is constructed by placing two separate heaters side-by-side along their width (Figure 5.3). The power ratings of the heaters are matched to ensure that they produce identical heat fluxes for a given power supply voltage. This ensures that a constant heat flux boundary condition is maintained over the entire length of the main heater.

The guard heaters are attached to the lower end of the base plate, exactly underneath the main heaters. The guard heaters used here are identical to the main heaters in terms of size, construction, and power rating. The purpose of the guard heater is to prevent the loss of heat from the primary heaters to the base plate and ensure that all the heat flux generated by the main heaters is supplied to the porous layer above. As mentioned above, a finite amount of heat loss always takes place through the base for a given temperature difference across the base plate. By employing a guard heater and suitably adjusting its power, the temperature difference across the base plate can be reduced to zero, thereby eliminating any heat loss through the base. If the guard heater is not employed, heat loss through the base will increase with rise in the boundary temperature and the heat flux supplied by the main heater to the convection cell will decrease as the experiment progresses.

\subsubsection{Top plate assembly}

The top plate assembly comprises the third component of the experimental apparatus. It consists of the top plate and the supporting plate (Figure 5.4). The main purpose of the top plate is to provide a constant temperature upper boundary that will set up an unstable temperature gradient across to convection cell and trigger the onset of convection. For the present experiments, a $21.59 \mathrm{~cm} \times 21.59 \mathrm{~cm} \times 0.32 \mathrm{~cm}$ copper plate is chosen as the top plate. This plate has a $0.64 \mathrm{~cm}$ DIA coiled copper tubing brazed to its top surface through which water can be circulated. Copper is chosen as the plate material due to its high thermal conductivity and the plate thickness is kept very small. This causes the thermal resistance across the plate thickness to be very small and ensures that rapid thermal equilibrium is achieved between the circulating water and the plate. 
(a)

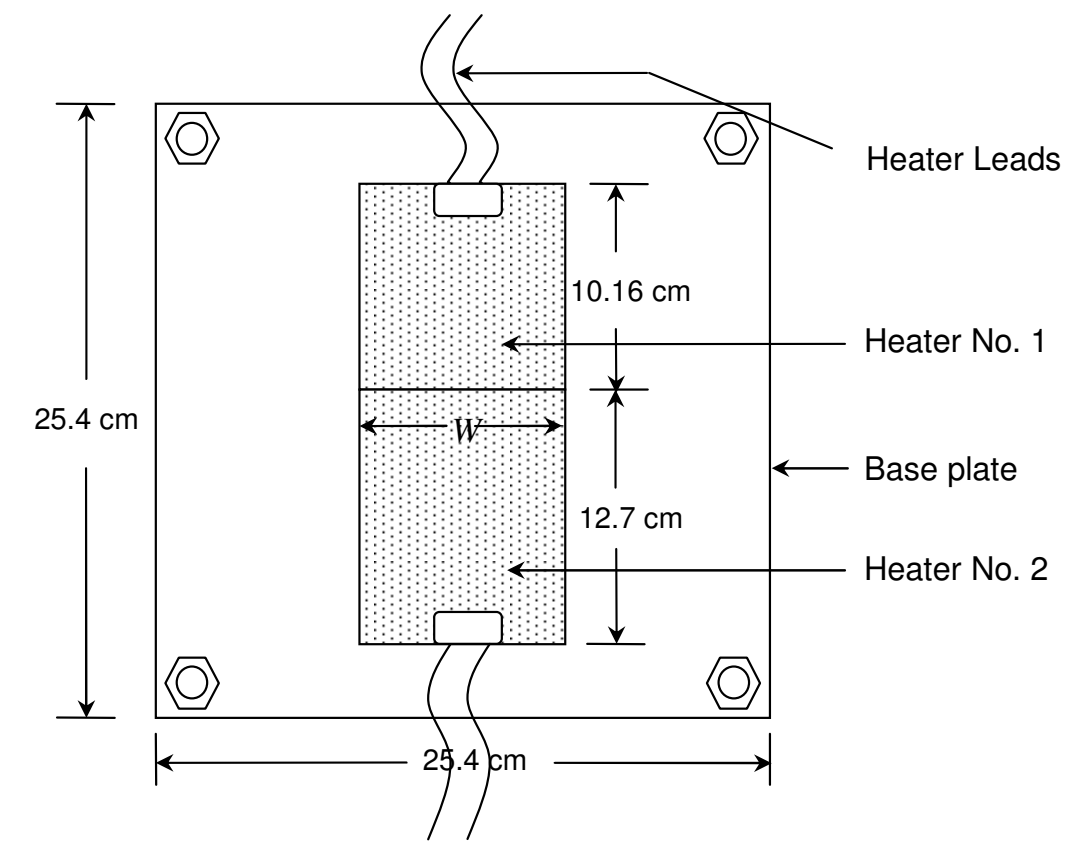

(b)

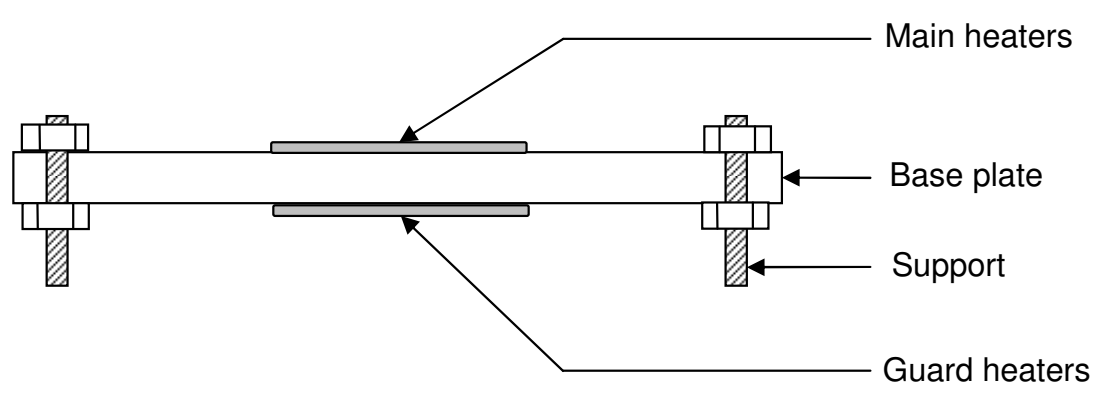

Figure 5.3: Schematic of the base plate assembly. (a) top view, and (b) side view.

The supporting plate forms the upper part of the assembly and its purpose is to hold the top plate in position. The cooling plate is attached to the supporting plate with three screw rods. As can be seen from Figure 5.4, this construction allows the distance between the top plate and the supporting plate, $H_{T}$, to be adjusted. In this way the working height and the aspect ratio for any particular experiment can be easily set depending on the requirements of that experiment. The supporting plate is also provided with a bleed hole to prevent any pressure build-up within the cell during the experiments. During assembly of the entire apparatus, it is important that the top plate assembly be easily and accurately mounted. This is enabled by machining a shallow groove on the lower surface of the supporting plate into which the side walls of the convection cell can be fit. 


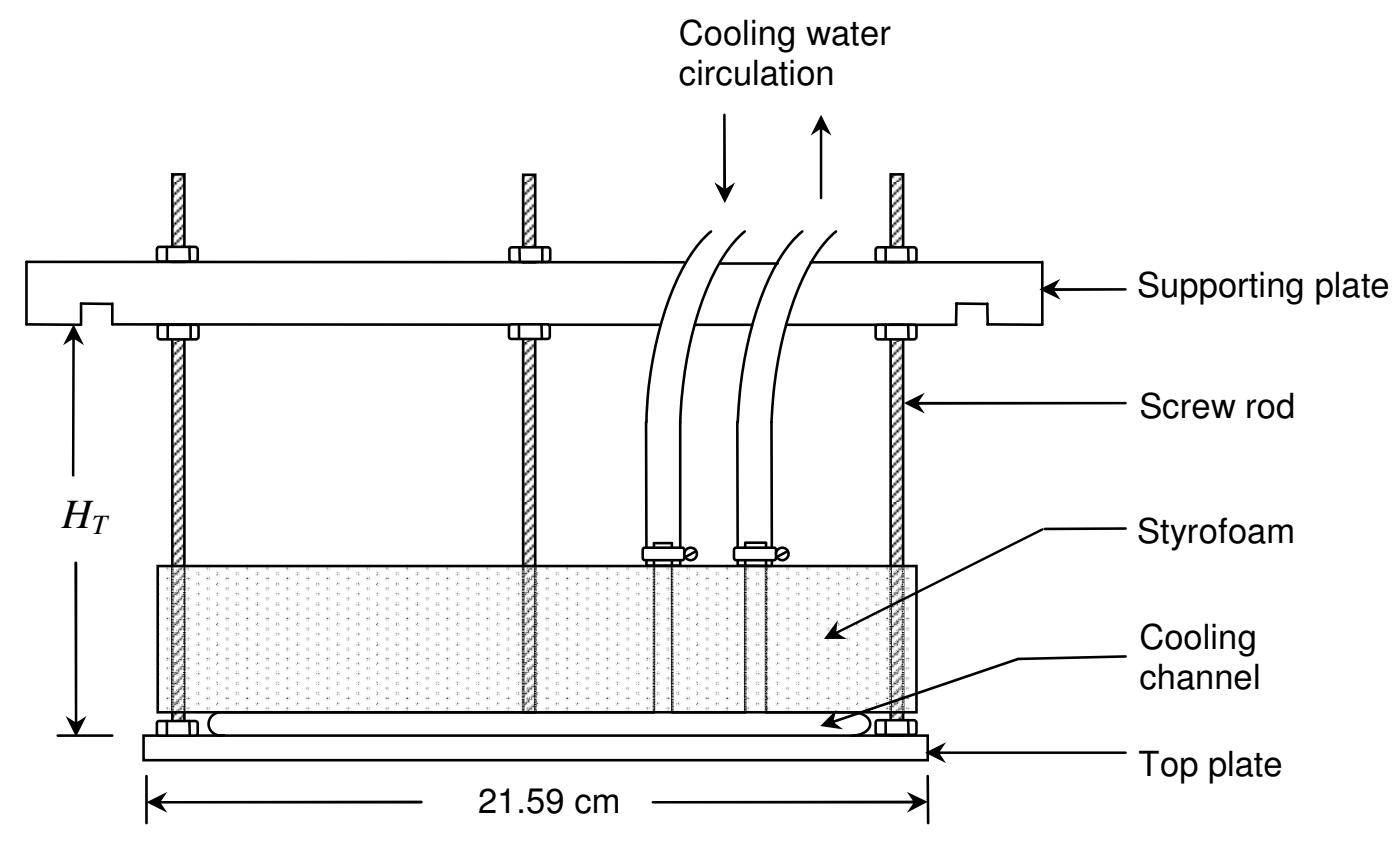

Figure 5.4: Schematic diagram of the top plate assembly showing the different components.

\subsubsection{Instrumentation and assembly}

The instrumentation used in the present experiments and the assembly of the overall experimental setup will be now discussed. This is a very crucial part of the experimental process as the accuracy of the instrumentation and overall assembly will directly influence the accuracy of the obtained results. In particular, close attention must be paid to the selection of the instrumentation and its incorporation into the experimental apparatus to ensure that the instrumentation does not interfere with the convective motion during the experiment. As discussed earlier, two primary quantities need to be measured during the experiments: temperatures at various locations inside the apparatus, and the power supply to the main heaters. All temperature measurements in the present study are made with Type E thermocouples (chromel-constantan). These couples are chosen for their high accuracy $\left(1.2{ }^{\circ} \mathrm{C}\right.$ or $0.75 \%$ error, whichever is highest) and ruggedness. The thermocouple junctions are made with the twist-and solder method. For increased accuracy, the Special Limits of Error grade of thermocouple wire is used. Two different sizes of thermocouple wires are used: $36 \mathrm{Ga}(0.127 \mathrm{~mm}$ DIA) wire for measuring the top plate temperatures, and $30 \mathrm{Ga}$ (0.254 mm DIA) wires for all other temperature measurements. Prior to attaching them, the accuracy of all the thermocouples is verified by measuring the ice point of water $\left(0{ }^{\circ} \mathrm{C}\right)$ in an ice bath. The maximum observed error is found to be $\pm 0.5^{\circ} \mathrm{C}$, well within the accuracy range of type $\mathrm{E}$ thermocouples.

For every experiment, temperature measurements are made at six different locations 
Table 5.2: Thermocouple locations at the different surfaces.

\begin{tabular}{lcl}
\hline Thermocouple location & Number & Position \\
\hline \hline Main heater surface & 9 & $\begin{array}{l}\text { Transverse and longitudinal centerlines } \\
\text { Transverse: } 2.2 \mathrm{~cm} \text { apart } \\
\text { Longitudinal: } 5.08 \mathrm{~cm} \text { apart }\end{array}$ \\
Guard heater surface & 5 & Zig-zag pattern: $6.35 \mathrm{~cm}$ apart \\
Top plate & 4 & Random locations \\
Inner walls & 4 & Center of wall, $0.19 \mathrm{~cm}$ above base \\
Outer walls & 4 & $\begin{array}{l}\text { Center of wall, } 0.19 \mathrm{~cm} \text { above base } \\
\text { Cavity mid-plane }\end{array}$ \\
& 9 & $\begin{array}{l}\text { Transverse and longitudinal centerlines } \\
\text { Transverse: } 2.2 \mathrm{~cm} \text { apart }\end{array}$ \\
& & Longitudinal: $3.81 \mathrm{~cm}$ apart \\
\hline
\end{tabular}

within the apparatus. These locations along with the number of thermocouples at each location are summarized in Table 5.2. Figure 5.5 shows the thermocouple locations on the top plate, main heaters, and the guard heaters. Five thermocouples are used to measure the top surface and the guard heater surface temperatures while a total of nine thermocouples are used to measure the main heater surface temperature. A larger number of thermocouples are used for the main heaters as the construction of the apparatus for the present experiments is such that a constant heat flux boundary condition exists on the heater surface as opposed to a constant temperature boundary condition, and thus the temperature on the main heater surface varies significantly, especially in the transverse direction. To understand this variation and accurately compute the average temperature across the heater surface, five thermocouples are mounted in both the lateral and transverse directions. It must be noted that while the guard heater too produces a constant flux, the temperature variation across the heater is not very high as the heater is operated at a very low power. As such, fewer thermocouples are required to monitor the guard heater temperature.

In addition to the top surface and the heater surfaces, temperatures are also measured along the inner and outer walls of the apparatus and along the vertical center-plane of the experimental domain. The thermocouples at the inner and outer walls of the convection cell are used for temperature measurements at these locations from which the heat loss through the sides of the apparatus can be calculated. Also, a total of nine different temperature measurements are made along the horizontal mid-plane of the cavity by attaching 
(a)

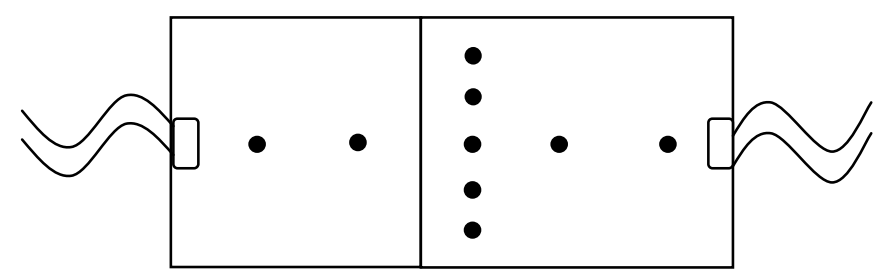

(b)

(c)
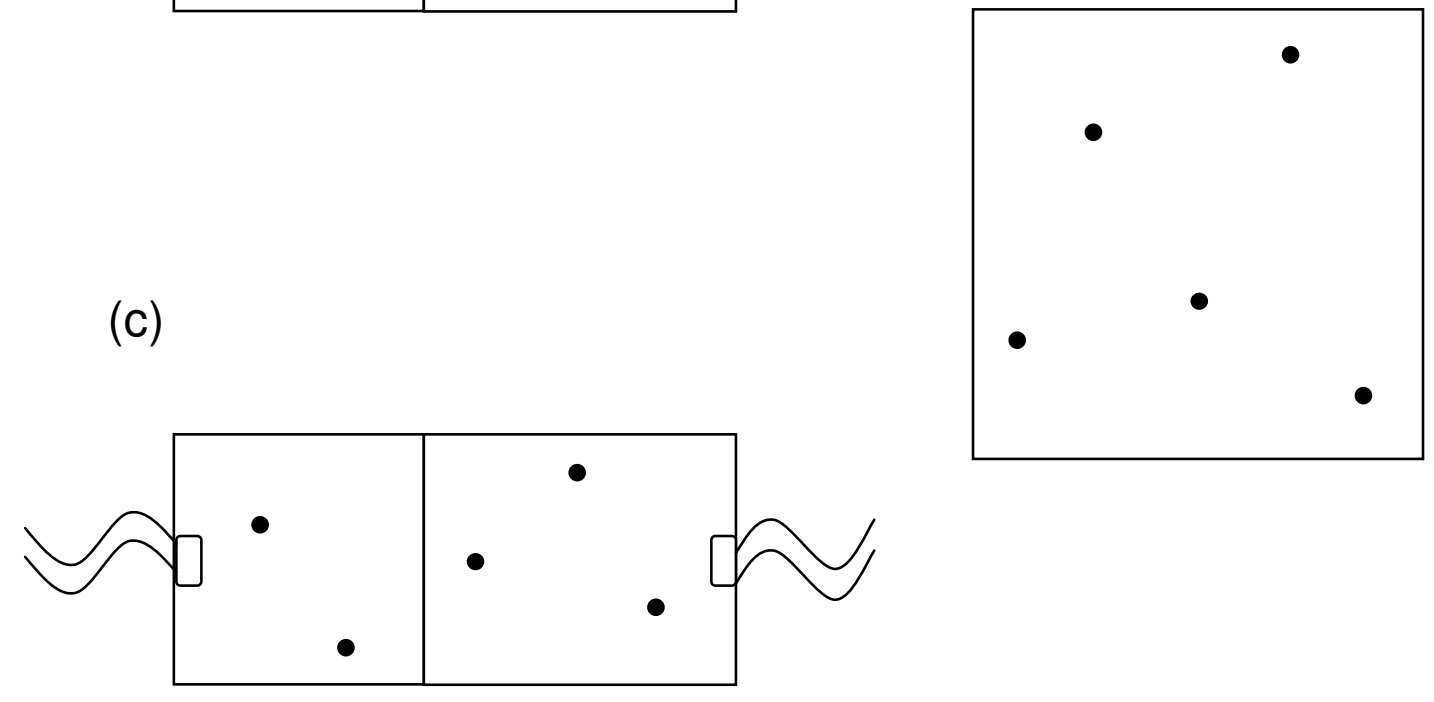

Figure 5.5: Thermocouple locations at (a) main heater surface, (b) top plate, and (c) guard heater surface.

thermocouples to a fixed grid. The grid is made from a $21.6 \mathrm{~cm} \times 1.6 \mathrm{~cm}$ polycarbonate frame to which two slightly stretched nylon wires are attached in the transverse and longitudinal directions along the center. The frame kept at a height of $1.9 \mathrm{~cm}$ above the base of the convection cell and is positioned by placing it on four corner posts that are glued to the inner walls of the cell at the four corners (Figure 5.6). The aim of these temperature measurements is to measure the size of the convective cells and compare them with the predictions from the numerical simulations. In addition, these measurements will aid in understanding the three-dimensional pattern of the convective motion. For the reason, the thermocouples are positioned differently in to two directions. In the longitudinal direction, the thermocouples are placed just above the heater surface as the bulk of the convection motion is expected to be in this region. In the transverse direction, the thermocouples are positioned evenly along the depth of the convection cell. The exact thermocouple positions are listed in Table 5.2. 
(a)

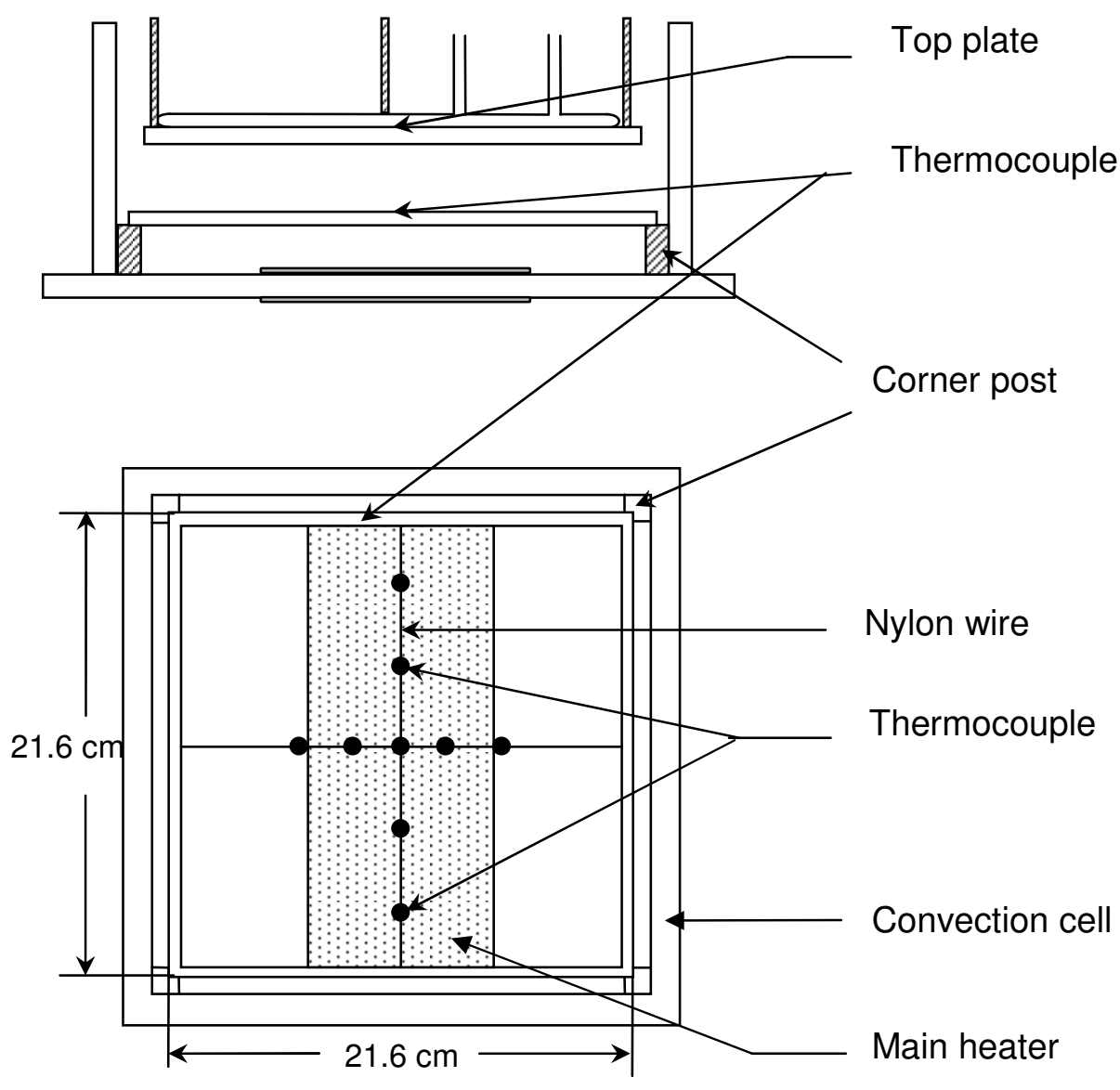

Figure 5.6: (a) Position of the thermocouple frame inside the cavity. (b) Thermocouple locations on the frame.

In all cases, the thermocouples are attached to the surfaces with high thermal conductivity epoxy (Omega Engineering Inc., Stamford, CT). In order to minimize interference with the experiment, the epoxy beads are kept as small as possible. This is particularly important in attaching the thermocouples to the main heater surface and the thermocouple frame where the size of the epoxy beads is kept smaller than the size of the glass beads. Similarly, in order to avoid interference, the thermocouple wires are taped to the base and side walls of the convection cell. The wires are run upwards along the side walls of the cell all the way up to the cell and are taken out through holes provided on the top supporting plate. The ends of the wires are connected to a digital data acquisition system (Model 34970A, Agilent Technologies, Santa Clara, CA) that is capable of handling over 50 thermocouple measurements. This data acquisition system converts the thermocouple voltage output to temperature readings and sends to the output directly to a PC, where the measurements 


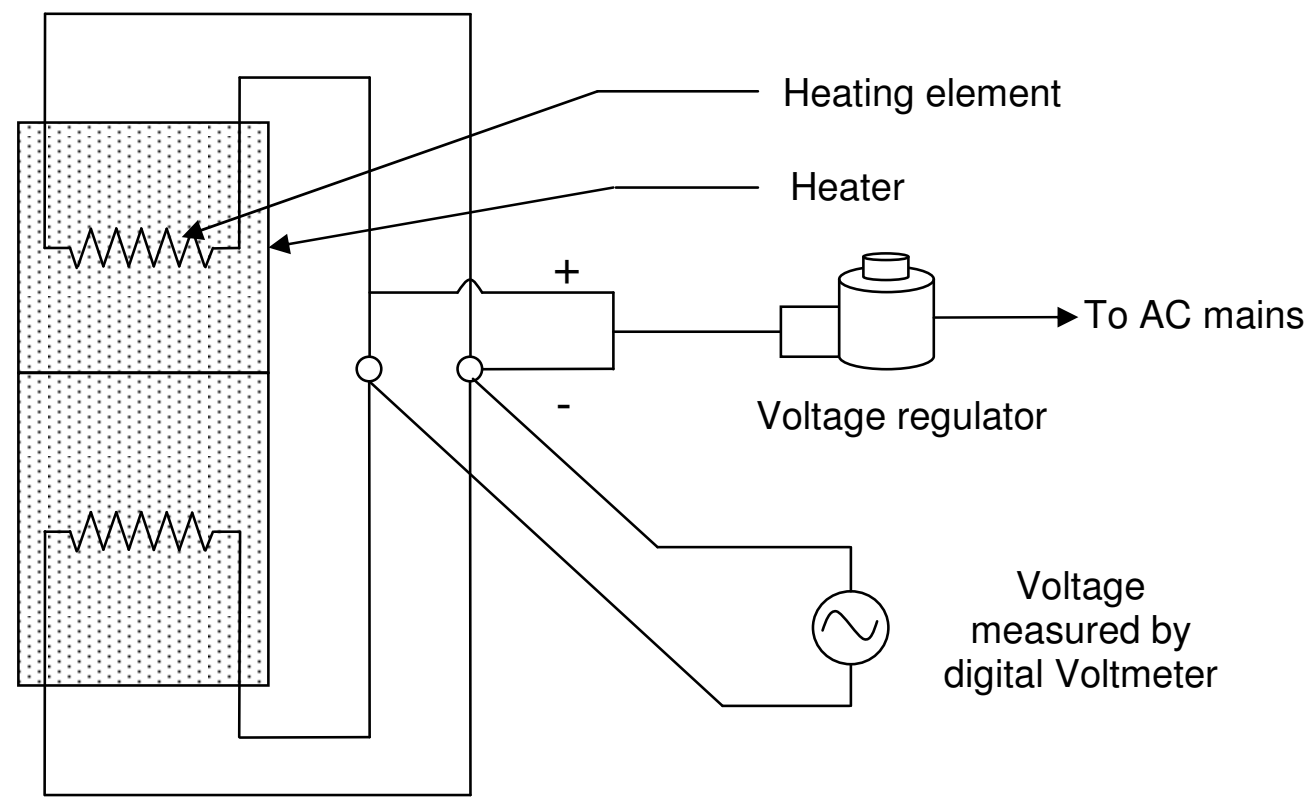

Figure 5.7: Power supply circuit to the main and guard heaters.

are recorded to the hard drive.

Power is supplied to the main and guard heaters through two separate variable voltage regulators which are connected to the main $\mathrm{AC}$ supply voltage line. With this arrangement, the power supply to main and guard heaters can be individually controlled. The heat flux produced by the main heaters is calculated by measuring the AC voltage across the heaters and using the resistance heating formula,

$$
q^{\prime \prime}=\frac{1}{A_{\text {tot }}}\left(\frac{V^{2}}{R_{1}}+\frac{V^{2}}{R_{2}}\right)
$$

where $V$ is the voltage drop across the two main heaters, $R_{1}$ and $R_{2}$ are the resistances of the heaters and $A_{\text {tot }}$ is the total area covered by the two heaters. The voltage drops across the heaters are measured by connecting two wires in parallel across the heater circuit to the data acquisition system. The measurement circuit is shown schematically in Figure 5.7. A schematic diagram of the final experimental setup is shown in Figure 5.8. For the final assembly, the apparatus is placed on a laboratory bench and is made horizontal by adjusting the screws on the four corner supports and checking with a spirit-level. The top plate is connected to the main water supply line and drain. Finally the apparatus is insulated on all sides using $5.08 \mathrm{~cm}$ thick Styrofoam boards. 


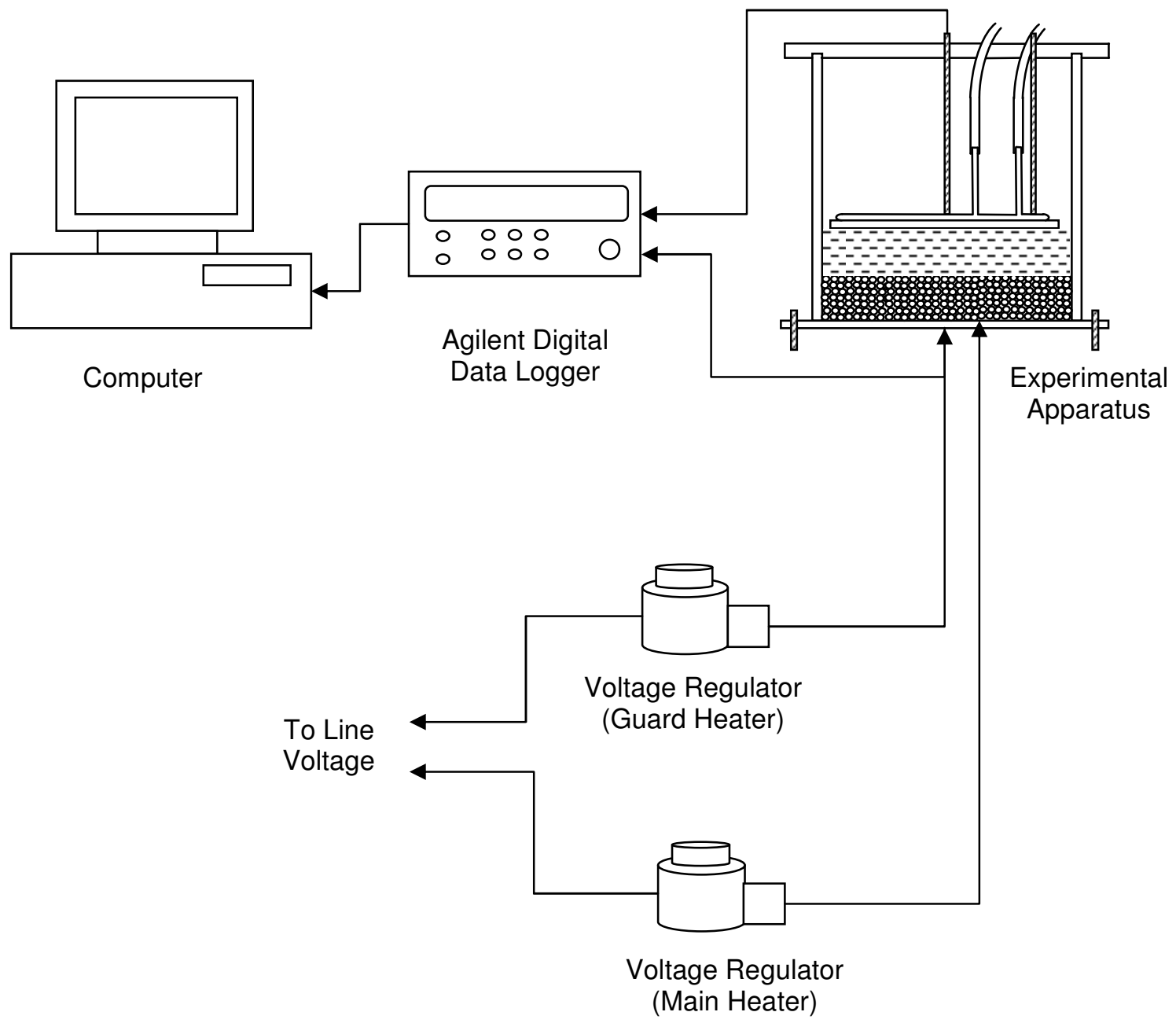

Figure 5.8: Overall experimental setup.

\subsection{Experimental procedure}

The experimental procedure comprises of several different steps which must be carefully performed in order to minimize the uncertainties in the obtained data. A number of these steps must actually be performed prior to each experiment. First, the glass beads used are thoroughly cleaned to ensure that no unwanted dust or debris end up in the apparatus. This cleaning is done on the day prior to the actual experiment. The beads are first washed with a mild soap and tap water. Thereafter, they are rinsed several times with tap water to ensure that all the soap is washed away and no residue is left behind. Finally, the beads are washed once with distilled water. The excess water is allowed to drain away and the beads are then left to dry overnight at room temperature.

On the day of the experiment, the beads are carefully inspected for traces of residual moisture that may have been trapped in them. In general, the beads were always found 
to be dry. On a few occasions when the beads are not completely dry, traces of residual moisture are removed by mildly heating them over and hot plate and then allowing them to cool. Prior to the beginning of an experiment, the inside of the apparatus is carefully cleaned with a moist cloth to remove any dust present. Thereafter, the instrumentation wires are carefully inspected to check for signs of tear or damage. After verification, the data acquisition system is turned on and the temperature and the voltage readings are checked for any anomalies. The acquisition system is allowed to run for $15 \mathrm{~min}$ after which the thermocouple readings are checked to ensure that the readings are close to room temperature which is measured separately with a mercury-in-glass thermometer.

After the initial checks are complete, de-gassing of the distilled water that is to be used as the saturating fluid is started. De-gassing the saturating fluid is very important in order to prevent the release and accumulation of air bubbles inside the apparatus during the experiment. In the present experiments, water is de-gassed by heating it in a metal pan over a hot plate up to its boiling point. After several trial runs it is seen that a total heating time of $45 \mathrm{~min}$ is sufficient to remove all the dissolved air. After de-gassing, the water is allowed to cool to room temperature. To accelerate the cooling process, coiled copper tubing with tap water running through it is placed inside the metal pan.

During the de-gassing of the water, the apparatus is prepared for the experiment by filling it with glass beads up to the desired height. To ensure uniform distribution of the beads, the apparatus is gently shaken by holding it at its base. For the present set of experiments it is very important that the interface between the glass beads and the overlying fluid layer is very nearly flat. To ensure a flat surface, a thick aluminum plate fitted with a screw rod is placed over the beads and is pressed down firmly in order to create a flat surface on top layer of the beads. Once the height of the porous layer and the flatness of the interface are checked, the beads are saturated with water by siphoning through a flexible tube that is attached to one of the inner walls of the convection cell. During filling of the tank, the aluminum plate is kept on top of the beads till after the porous layer is saturated with water. This ensures that no defects are created on the top layer during the filling process and an almost flat interface exists between the porous layer and the overlying fluid layer. Once the beads are saturated with water, the aluminum plate is removed and the cell is filled up to its working height. The top plate assembly is then slowly lowered into the cell and placed on top of the fluid layer. Placement of the top plate over the fluid layer is done very carefully to avoid trapping any air bubbles under the plate.

Once the top plate is in place, the circulation of tap water through the coils of the top plate is started. The apparatus is checked one final time to ensure that there are no leaks or loose wire connections. The temperature readings on the data acquisition system too are checked for any discrepancies. The Styrofoam insulation boards are then placed in 
position around the sides and the base of the apparatus and are held in place by adhesive tape. The experiment is started by switching on the power supply to the main and guard heaters. During the initial part of each experimental run, the base and guard temperatures are closely monitored to ensure that the average surface temperatures of both the heaters are very close to each other. The voltage regulators for both heaters are then adjusted accordingly. Usually this is done during the first fifteen minutes of each run as the largest changes in heater temperatures occur during this time. After this the experiment is allowed to run till it reaches steady-state. During this time, the temperature readings on the main and guard heaters, as well as the top plate, are monitored periodically. Steady-state is assumed to have been attained when the average heater and top surface temperatures do not change by more than $0.5 \%$ over a five minute time period. The time to attain steadystate depends primarily on the Rayleigh number with shorter times being required at higher Rayleigh number. In general, steady-state is achieved within 35-40 min although for some cases it is about $90 \mathrm{~min}$.

Once the system has attained steady-state, the temperature readings are stored on the computer and the experiment is stopped by turning off the power supply to the heaters. The insulation is removed and the system is allowed to cool to room temperature. During this time, the water circulation through the top plate is allowed to continue to enable the system to cool faster. In general the cooling time is found to be no more than 15-20 min during which the system temperatures are monitored. Once cooling is done, a new experiment is started following the same procedure described above. 


\section{Chapter 6}

\section{Experimental Results}

In this chapter, results from the experimental study are presented. Because the main aim of the experiments is to obtain the overall Nusselt-versus-Rayleigh number relations for different heater lengths and fluid-to-porous layer height ratios, it is important that these quantities, along with their net uncertainties, be calculated accurately. This chapter therefore begins with a detailed description of the data analysis procedure adopted in this study. Thereafter, the experimental data is presented. Finally, the results are discussed in detail.

\subsection{Data analysis procedure}

\subsubsection{Data reduction procedure}

During each experiment, the digital data acquisition system generates an ASCII file that contains all the data recorded during every measurement: the temperatures measured by the thermocouples, the supply voltages for the main and guard heaters, and the time point for each measurement. To perform the data reduction, two different MATLAB ${ }^{\circledR}$ (Mathworks Inc., Natick, MA), scripts have been developed. These scripts take the ASCII files generated by the data acquisition system and together perform the following procedure:

1. Calculate steady-state values: The steady-state value for each quantity measured during a particular experimental run is computed by taking an arithmetic mean of the steady-state data over a 4 minute time period or over the final 24 time point measurements. In order to estimate the error in the steady-state measurements, the standard deviation is also computed.

2. Average temperatures for the main heater, guard heater, and the top plate are computed by taking an arithmetic mean of all the thermocouple measurements for each surface. The standard deviation of the measurements is also computed. 
3. The heat supplied by the primary heater is calculated using the formula

$$
q=\frac{V^{2}}{R_{\mathrm{eff}}}
$$

where $R_{\text {eff }}$ is the effective parallel resistance of the two heaters and is,

$$
R_{\mathrm{eff}}=\frac{1}{R_{1}}+\frac{1}{R_{2}}
$$

4. Heat losses through the base and side walls are calculated. This is discussed in detail in the next section.

5. The overall Nusselt number is calculated from the formula

$$
N u=\frac{\left(q-q_{\mathrm{loss}}\right) H}{k_{\mathrm{f}} A_{\text {tot }}\left(T_{H, \mathrm{avg}}-T_{C, \mathrm{avg}}\right)} .
$$

6. The overall Rayleigh number is calculated from the formula

$$
R a=\frac{g \beta H^{3}\left(T_{H, \mathrm{avg}}-T_{C, \mathrm{avg}}\right)}{\nu \alpha_{\mathrm{f}}}
$$

7. Uncertainty analysis is performed to determine the overall uncertainties in the values of the Nusselt and Rayleigh numbers. This is described in detail in Appendix D.

As can be seen, all the required quantities can be calculated directly from the experimental data using the above procedure. The only quantity that needs to be carefully estimated the net heat loss from the apparatus. This is described next.

\subsubsection{Estimation of heat losses}

A crucial component of the data analysis procedure is the accurate estimation of the heat loss occurring from the experimental apparatus. Since the losses are, to an extent, unavoidable, correct estimation of the overall loss is important for calculating the Nusselt number and for reducing the overall uncertainty in the measurements. For the given apparatus, the two main sources of heat loss are: a) losses through the side walls, and b) heat loss through the base. In order to calculate these losses, data from the temperature measurements are used.

To estimate the heat loss through the side walls, a one-dimensional conduction analysis is used. As mentioned in the previous chapter, a total of eight thermocouples - 


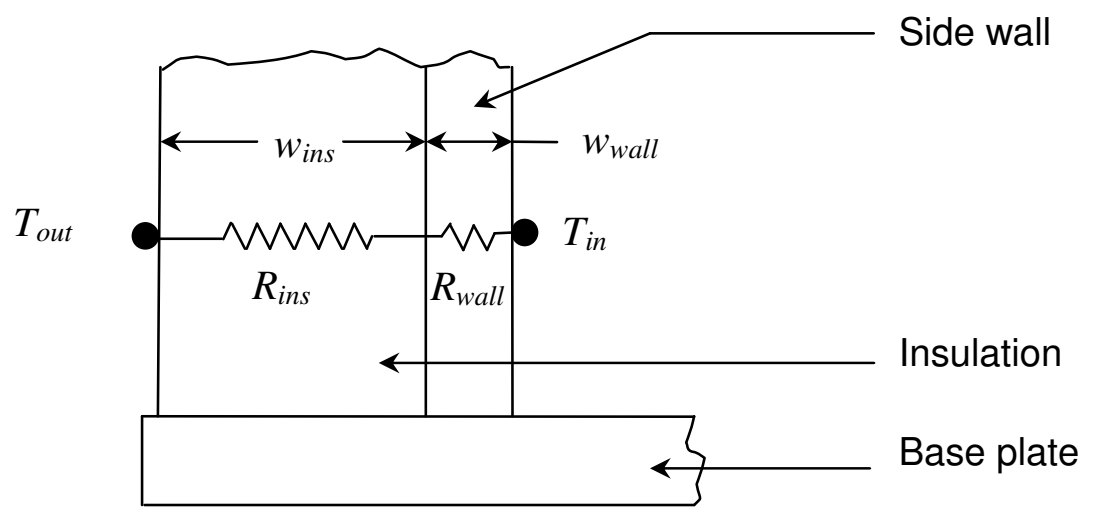

Figure 6.1: Thermal resistance network for the side wall.

four on the inner surface of the side walls (one on each wall), and four on the outer surface of the insulation boards - are specifically assigned for this purpose. For each pair of inner wall-outer wall thermocouples a conduction resistance network can be constructed. This is shown schematically in Figure 6.1. As can be seen, the thermal resistances of the side wall and the insulating board form a series resistance circuit, for which the effective resistance is,

$$
\begin{aligned}
R_{\text {side }} & =R_{\text {ins }}+R_{\text {wall }} \\
& =\left(\frac{w_{\text {ins }}}{A_{\text {ins }} k_{\text {ins }}}+\frac{w_{\text {ins }}}{A_{\text {ins }} k_{\text {ins }}}\right) .
\end{aligned}
$$

Based on the theory of thermal resistances, the heat loss though the side wall is,

$$
q_{\mathrm{ls}}=\frac{T_{\mathrm{in}}-T_{\mathrm{out}}}{R_{\text {side }}}
$$

where $T_{\text {in }}$ and $T_{\text {out }}$ are the temperatures on the inner wall of the apparatus and the outer wall of the insulation, and $q_{\mathrm{ls}}$ is the heat loss though a single side wall. The total heat loss through the four side walls can then be written as,

$$
q_{\text {side }}=\frac{1}{R_{\text {side }}} \sum_{i=1}^{4}\left[\left(T_{\mathrm{in}}\right)_{i}-\left(T_{\text {out }}\right)_{i}\right]
$$

This provides a direct and simple way to estimate the side wall heat loss.

Estimation of the heat loss through the base plate is slightly more involved. This is because heat loss through the base plate occurs both in the transverse as well as the lateral direction. To better understand the nature of heat transfer in the base plate, a 
(a)

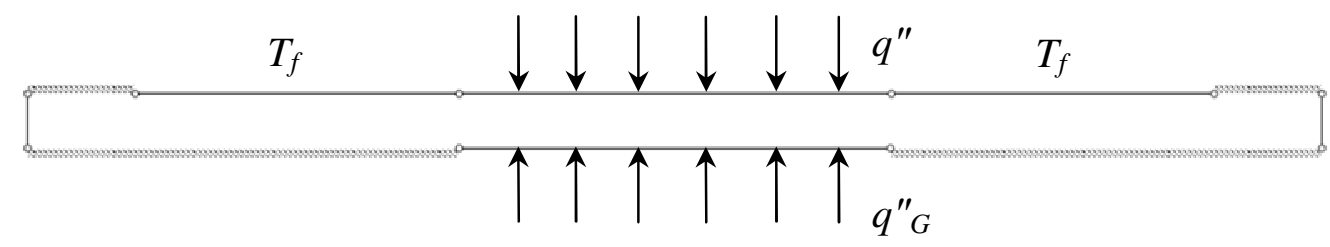

(b)

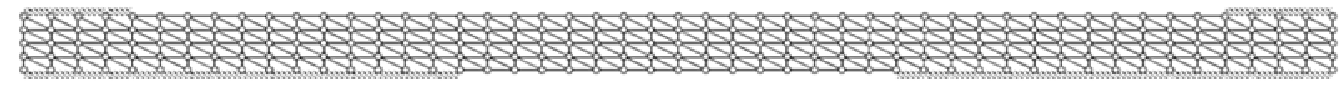

(c)

(d)

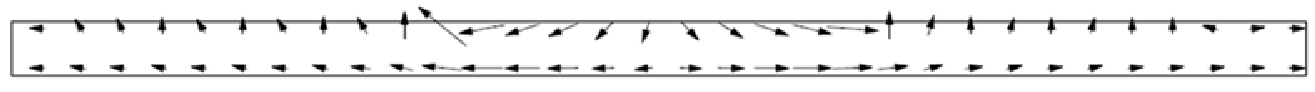

Figure 6.2: Finite-element simulation of the heat transfer problem in the base plate. (a) Problem setup and boundary conditions, (b) finite-element grid used, (c) steadystate temperature contours, and (d) heat flow lines.

finite element simulation of the conduction problem in the plate is performed. This will enable a better understanding of the direction of heat losses and their magnitude. For the simulation, a two-dimensional problem is selected with the following parameters:

- Length of plate: $30.38 \mathrm{~cm}$.

- Thickness of the plate: $1.27 \mathrm{~cm}$.

- Main heater: $10.16 \mathrm{~cm}, 25 \mathrm{~W} / \mathrm{m}^{2}$ heat flux.

- Guard heater: $10.16 \mathrm{~cm}, 2.5 \mathrm{~W} / \mathrm{m}^{2}$ heat flux.

- Top surface, non-heated section: $T_{\mathrm{f}}=25^{\circ} \mathrm{C}$.

- Side edge: $T_{\infty}=25^{\circ} \mathrm{C}$.

The results from the simulations are shown in Figure 6.2. As can be seen, at steady-state, the temperature field along the center of the plate is uniform indicating a very small temperature gradient across the plate thickness. This is to be expected based on the fact that the presence of the guard heater neutralizes the heat loss through the bottom of the main heater. Across the plate length the isotherms are nearly vertical indicating the presence of a lateral temperature gradient. This can be better seen by considering the 


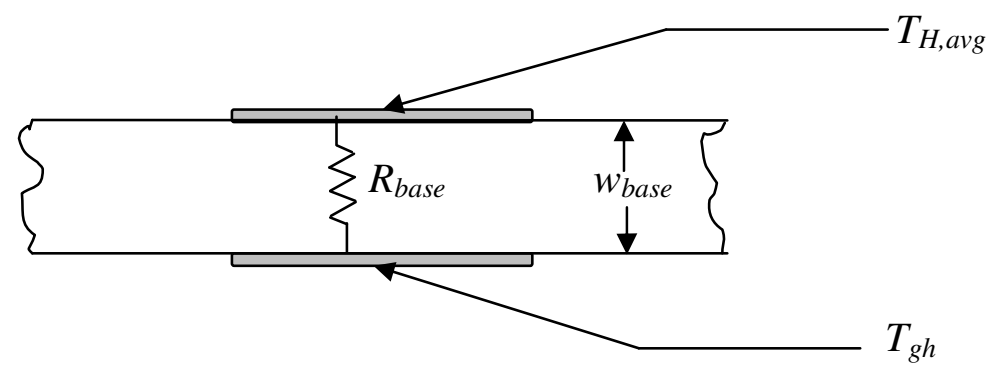

Figure 6.3: Thermal resistance network for the base plate.

temperature gradients in the plate as shown in Figure 6.2(d). Except near the centerline of the plate, where there is some heat flow in the transverse direction, the bulk of the heat flow in the plate is in the lateral direction. A simple technique for calculating the heat losses in both directions is to break up the two-dimensional problem into two one-dimensional problems. With this approach the heat losses in the transverse and lateral directions are treated independently of one another.

First, the method for calculating the transverse heat loss is discussed. A onedimensional conduction model is used to estimate the heat loss through the base based on the temperature measurements on the main and guard heater surface. Consider the schematic diagram of the base plate heater assembly, as shown in Figure 6.2. Based on a thermal resistance model, the heat lost through the base is,

$$
\begin{aligned}
q_{l b} & =\frac{T_{H, \mathrm{avg}}-T_{G, \mathrm{avg}}}{R_{\mathrm{base}}} \\
& =\frac{A_{\mathrm{tot}} k_{\mathrm{base}}\left(T_{H, \mathrm{avg}}-T_{G, \mathrm{avg}}\right)}{w_{\mathrm{base}}},
\end{aligned}
$$

where $T_{H \text {,avg }}$ and $T_{G \text {,avg }}$ are the average surface temperatures of the main and guard heaters, respectively. It must be noted here that the thermocouples for measuring the heater temperatures are located on top of heater surface. As a result, in writing this equation, it has been assumed that the thermal resistances of the main and guard heaters are negligible. This is a reasonable assumption considering the very small thickness of the heaters (1.4 $\mathrm{mm})$. Also, since the heaters have a heating element sandwiched between two silicone rubber sheets that provides a constant heat flux, it is reasonable to assume that the entire heater is isothermal across its cross-section. It may be recalled that the primary reason behind installing the guard heater is to actually prevent any heat loss through the base by eliminating the temperature gradient in the transverse direction. As such, heat loss in the transverse direction should be zero. However, since it is not possible to exactly match the 
main and guard heater temperatures during an experiment, a small but finite heat loss does take place during each run. This is estimated with equation (6.8).

Next, the lateral heat loss through the base plate is calculated. Based on a onedimensional conduction model, the lateral heat loss can be written as,

$$
q_{l}=2\left[\frac{A_{\mathrm{cs}} k_{\text {base }}\left(T_{\mathrm{cl}}-T_{\infty}\right)}{l_{\text {base }} / 2}\right]
$$

Here $A_{\mathrm{cs}}$ is the cross-sectional area of the base plate $T_{\mathrm{cl}}$ is the average centerline temperature across the plate thickness. Since there is no direct method for measuring the temperature across the centerline of the base plate, $T_{\mathrm{cl}}$ is assumed to be equal to the average temperature along the centerline (along the length of the heater) of the main heater surface. This is a reasonable assumption considering that the temperature across the plate thickness is almost uniform in the center due to the presence of the guard heater. Also, because there are four thermocouples mounted along the main heater centerline, the average temperature across the heater can be estimated with reasonable accuracy.

An important observation that can be made from the results of the finite-element simulation is the presence of large temperature gradient near the edges of the main heater. The heat flow lines in Figure 6.4(d) clearly show that a part of the heat lost by the main heater makes its way back into the convection cell near the edges of the heater. This means that the surface of the base plate outside the heater is not truly adiabatic. It also indicates that the lateral heat loss is less than what is estimated with equation (6.9). From the finite-element simulations, the heat leaking back into the convection cells is $\sim 40 \%$ of the lateral heat loss (actual 38.4\%). In terms of the total energy balance of the system however this is an extremely small value. For most experiments, the lateral heat loss is less than $1 \%$ of the total energy supplied by the main heaters. As such, the heat leaking back into the system is only a very small fraction of the total energy input and can be neglected without introducing significant errors in the overall heat transfer calculations. Therefore in the present calculations, the heat leakage into the system near the edges of the heater is neglected.

\subsection{Experimental Results}

\subsubsection{Validation of the experimental setup}

To validate the present experimental setup and the data reduction procedure, two different sets of experiments were performed, each with a different heater size. Experiments were performed for a fully porous layer $(\eta=0)$ with heater length ratios $\delta=0.44$ and 0.11. Each experiment was repeated thrice to ensure repeatability of the results. Results 
Table 6.1: Parameters used for studies on validation of experimental setup.

\begin{tabular}{cccc}
\hline Parameter & Present Study & Rajen and Kulacki [60] & Lai and Kulacki [61] \\
\hline Height ratio, $\eta$ & 0 & 0 & 0 \\
Heater length ratio, $\delta$ & $0.44,0.11$ & 0.5 & 0.14 \\
Aspect Ratio, $A$ & 6 & 6 & 21 \\
Darcy number, $D a$ & $4.04 \times 10^{-6}$ & $2 \times 10^{-6}$ & $3.4 \times 10^{-6}$ \\
\hline
\end{tabular}

for the experiments are show in Figure 6.4 along with comparison with the numerical and experimental results of Rajen and Kulacki [60] and Lai and Kulacki [61]. In these results, the Nusselt number, $N u_{\mathrm{m}}$, is based on the effective stagnant conductivity, $k_{\mathrm{m}}$, and the Rayleigh number, $R a_{\mathrm{m}}^{*}$, is the flux based porous media Rayleigh number which are defined as,

$$
\begin{gathered}
N u_{\mathrm{m}}=\frac{q_{\mathrm{net}}^{\prime \prime} H}{k_{\mathrm{m}}\left(T_{H, \mathrm{avg}}-T_{C, \mathrm{avg}}\right)}, \\
R a=\frac{g \beta K H^{2} q_{\text {net }}^{\prime \prime}\left(T_{H, \mathrm{avg}}-T_{C, \mathrm{avg}}\right)}{\nu \alpha_{\mathrm{m}} k_{\mathrm{m}}},
\end{gathered}
$$

where $q_{\text {net }}$ is the net heat flux supplied by the heaters and all other quantities have their usual meanings. Details of the comparison studies are listed in Table 6.1.

Comparison of the present experimental results with those of Rajen and Kulacki [60] is shown in Figure 6.4. As can be seen, agreement is very good with both the numerical and experimental results except at very low Rayleigh numbers where current results are lower than the numerical predictions. Although error bars are not shown here, it will be seen in the next section that the maximum uncertainty in the Nusselt number occurs at low Rayleigh numbers which can explain the observed discrepancy. Beyond the critical point, agreement with the numerical simulations is very good over the entire range of Rayleigh number with a maximum difference of $\sim 4.5 \%$ at $R a_{\mathrm{m}}^{*}=620$. The present results also agree well with the experimental results of Rajen and Kulacki though the difference between them is higher than. The maximum difference is about $7 \%$. This is to be expected considering that the heater length ratio used in the present experiments $(\delta=0.44)$ is slightly higher than that used by Rajen and Kulacki $(\delta=0.5)$.

Figure 6.5 shows the comparison of the present results for $\delta=0.11$ with the numerical and experimental results of Lai and Kulacki [61]. Again, there is some discrepancy in 


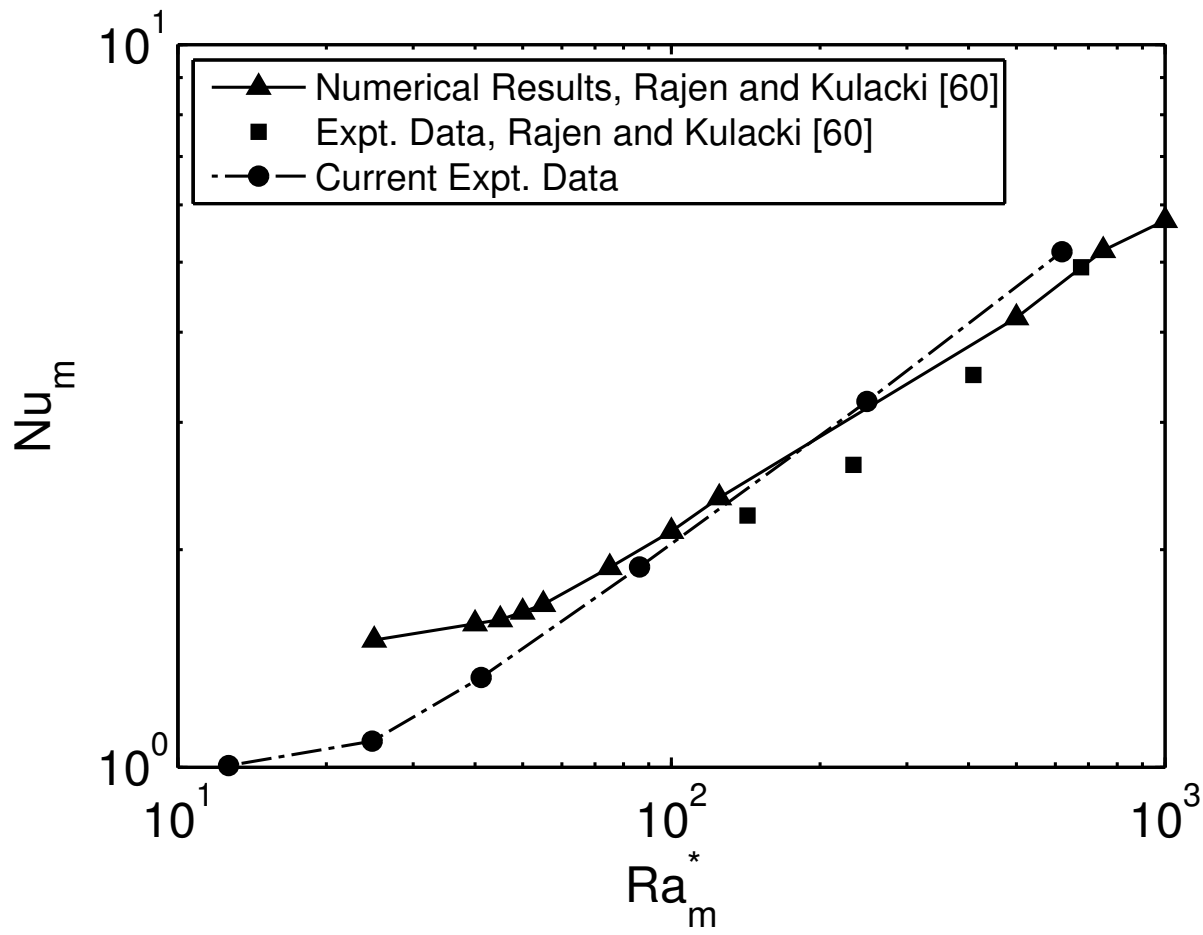

Figure 6.4: Comparison of current experimental data with the numerical and experimental results of Rajen and Kulacki [60].

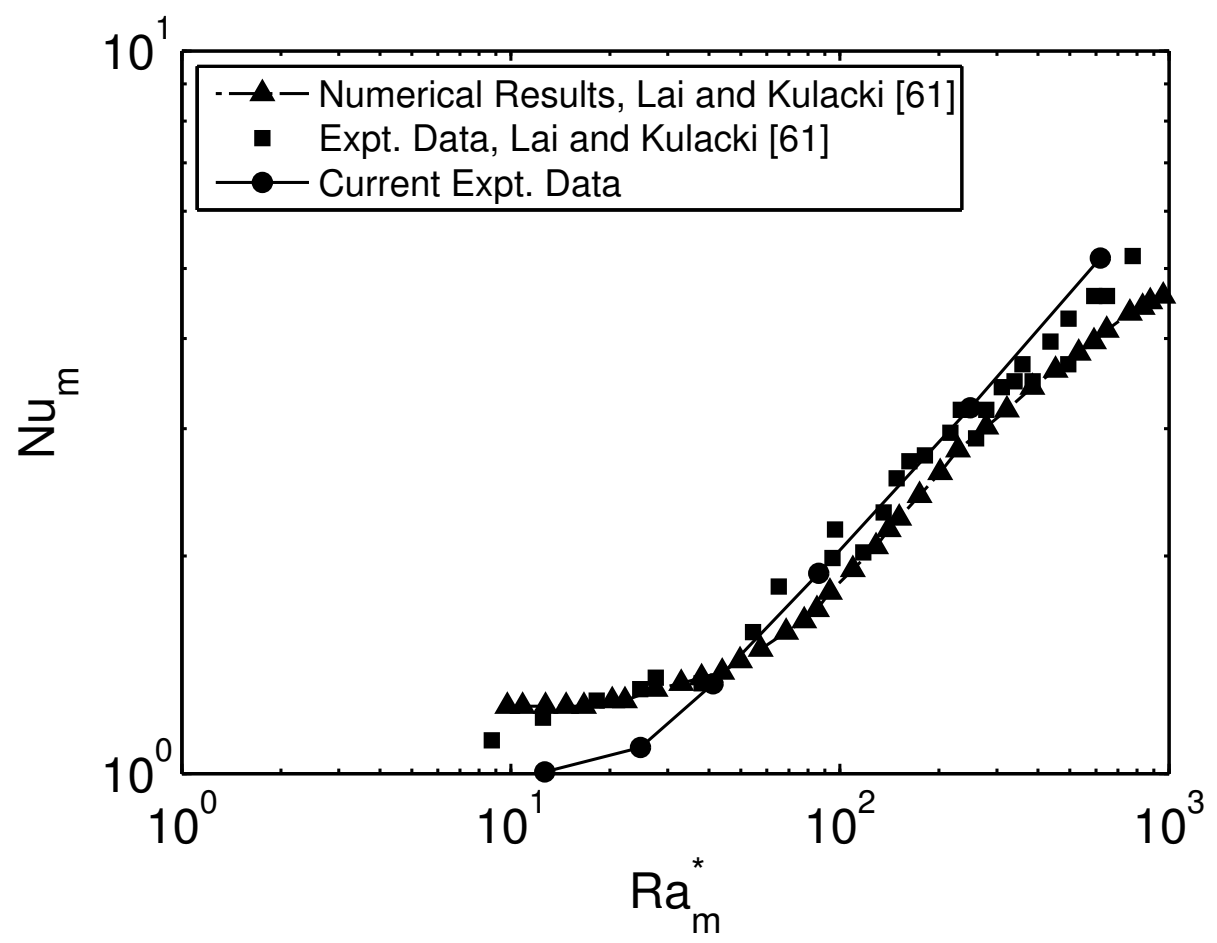

Figure 6.5: Comparison of current experimental results with the numerical and experimental results of Lai and Kulacki [61]. 
the two sets of results near the onset point. Beyond the critical point, however, the agreement is very good over the entire range of Rayleigh numbers. It must be noted that the heater length ratio in the present study is slightly smaller than that used by Lai and Kulacki [61]. The results also agree well with the results of their numerical simulations although the present numerical results consistently over-predict the Nusselt number by about $6 \%$.

\subsubsection{Heat transfer results}

Heat transfer results for convection in fluid-superposed porous layers will now be discussed. Table 6.2 lists all the primary measured parameters, and Table 6.3 gives the reduced heat transfer rates in terms of overall Nusselt and Rayleigh numbers along with their uncertainties. The overall Nusselt-versus-Rayleigh number data for all the experiments are shown in Figures 6.5 to 6.8 along with the best fit lines. The error bars on the graphs show the net uncertainties in evaluating these parameters. As can be seen, the heat transfer data on all the graphs can be correlated by straight lines on logarithmic coordinates indicating a power-law relationship of the form $N u=C R a^{n}$, which is agreement with the predictions of the numerical simulations. It also agrees with the established result that heat transfer data for natural convection problems that are derivatives of the Rayleigh-Bénard problem always follows a power-law relationship. Correlations of heat transfer data for the experiments are given in Table 6.4 .

A better understating of the experimental results can be obtained by considering Figure 6.9 which shows the best-fit correlations for the three porous layer height ratios, $\eta$, studied here. As can be seen, the overall Nusselt number for a given Rayleigh number increases with a decrease in height ratio. This is in agreement with the predictions of the numerical simulations. Also, the curves for $\eta=0.5$ and $\eta=0.75$ are nearly parallel. This too is in agreement with the predictions of the numerical simulations. The curve for $\eta=0.67$, however, shows some anomalies. At lower Rayleigh numbers, the Nusselt numbers of $\eta=0.67$ and $\eta=0.75$ are almost identical. This can be explained by considering uncertainties in the experimental measurements. As can be seen from Figures 6.6 and 6.7 the uncertainties in the Nusselt number at the lower end of the Nu-Ra curve are the highest. In addition, from an experimental perspective the difference between $\eta=0.67$ and $\eta=0.75$ is only about one bead diameter. Because the uncertainty in the height of the porous layer is one bead diameter, overlap of the Nusselt number is highly likely and is most likely the reason why the slope of the $\eta=0.67$ curve is somewhat different than the other two cases.

The effect of the heater length ratio on the overall heat transfer rates is shown in Figure 6.8. It can be seen that the Nusselt number increases with a decrease in the heater length ratio, $\delta$, from 0.44 to 0.11 . This trend too is in agreement with the predictions of the numerical simulations. This increase is however only clearly evident at higher Rayleigh 
Table 6.2: Measured parameters for all experiments.

\begin{tabular}{|c|c|c|c|c|c|c|}
\hline Run No. & $\delta$ & $\eta$ & $\Delta T\left({ }^{\circ} \mathrm{C}\right)$ & $q_{\text {main }}(\mathrm{W})$ & $q_{\text {net }}(\mathrm{W})$ & $q_{\text {net }}^{\prime \prime}\left(\mathrm{W} / \mathrm{m}^{2}\right)$ \\
\hline 1 & \multirow{7}{*}{0.44} & \multirow{7}{*}{0.5} & 5.07 & 4.05 & 4.16 & 179.42 \\
\hline 2 & & & 11.05 & 13.55 & 13.67 & 588.88 \\
\hline 3 & & & 15.06 & 28.97 & 29.22 & 1258.31 \\
\hline 4 & & & 19.84 & 61.01 & 61.27 & 2638.17 \\
\hline 5 & & & 26.61 & 112.57 & 112.81 & 4857.35 \\
\hline 6 & & & 30.41 & 138.47 & 138.48 & 5962.67 \\
\hline 7 & & & 36.03 & 202.15 & 202.28 & 8709.6 \\
\hline 1 & \multirow{7}{*}{0.44} & \multirow{7}{*}{0.67} & 5.59 & 4.33 & 4.16 & 179.33 \\
\hline 2 & & & 12.22 & 15.85 & 15.56 & 670.28 \\
\hline 3 & & & 15.78 & 30.6 & 30.35 & 1306.74 \\
\hline 4 & & & 21.22 & 67.93 & 68.15 & 2934.43 \\
\hline 5 & & & 25.5 & 105.71 & 106.81 & 4598.97 \\
\hline 6 & & & 31.32 & 157.07 & 157.46 & 6779.77 \\
\hline 7 & & & 36.51 & 211.96 & 212.15 & 9134.45 \\
\hline 1 & \multirow{6}{*}{0.44} & \multirow{6}{*}{0.75} & 6.04 & 4.38 & 4.03 & 173.86 \\
\hline 2 & & & 11.15 & 13.15 & 12.07 & 519.68 \\
\hline 3 & & & 16.16 & 26.41 & 26.1 & 1124.09 \\
\hline 4 & & & 20.6 & 45.18 & 44.79 & 1928.87 \\
\hline 5 & & & 27.24 & 76.8 & 76.36 & 3288.05 \\
\hline 6 & & & 37.13 & 123.8 & 123.25 & 5306.65 \\
\hline 1 & \multirow{8}{*}{0.11} & \multirow{8}{*}{0.5} & 6.4 & 1.85 & 1.82 & 314.82 \\
\hline 2 & & & 12.26 & 4.36 & 4.31 & 742.19 \\
\hline 3 & & & 17.29 & 8.5 & 8.36 & 1440.41 \\
\hline 4 & & & 21.32 & 13.58 & 13.38 & 2305.56 \\
\hline 5 & & & 26.87 & 22.1 & 21.93 & 3777.44 \\
\hline 6 & & & 31.51 & 30.27 & 30.04 & 5174.88 \\
\hline 7 & & & 36.49 & 39.45 & 39.15 & 6744.14 \\
\hline 8 & & & 41.75 & 50.17 & 49.62 & 8545.88 \\
\hline
\end{tabular}


Table 6.3: Reduced heat transfer data for all experiments.

\begin{tabular}{|c|c|c|c|c|c|c|}
\hline Run No. & $\delta$ & $\eta$ & $R a \times 10^{-7}$ & $N u$ & $U_{R a}(\%)$ & $U_{N u}(\%)$ \\
\hline 1 & \multirow{7}{*}{0.44} & \multirow{7}{*}{0.5} & 0.54 & 2.22 & 16.01 & 16.91 \\
\hline 2 & & & 1.36 & 3.32 & 8.82 & 7.87 \\
\hline 3 & & & 2.06 & 5.17 & 7.44 & 6.04 \\
\hline 4 & & & 3.07 & 8.18 & 6.63 & 4.99 \\
\hline 5 & & & 4.83 & 11.12 & 6.07 & 4.27 \\
\hline 6 & & & 5.93 & 11.89 & 5.9 & 4.03 \\
\hline 7 & & & 8.05 & 14.54 & 5.73 & 3.8 \\
\hline 1 & \multirow{7}{*}{0.44} & \multirow{7}{*}{0.5} & 0.6 & 2.01 & 14.69 & 15.69 \\
\hline 2 & & & 1.59 & 3.4 & 8.3 & 7.23 \\
\hline 3 & & & 2.26 & 5.11 & 7.27 & 5.84 \\
\hline 4 & & & 3.38 & 8.49 & 6.48 & 4.79 \\
\hline 5 & & & 4.62 & 10.96 & 6.13 & 4.34 \\
\hline 6 & & & 6.34 & 13.1 & 5.72 & 3.78 \\
\hline 7 & & & 8.23 & 15.04 & 5.86 & 3.99 \\
\hline 1 & \multirow{6}{*}{0.44} & \multirow{6}{*}{0.5} & 0.7 & 1.79 & 13.75 & 14.96 \\
\hline 2 & & & 1.43 & 2.89 & 8.76 & 7.93 \\
\hline 3 & & & 2.35 & 4.29 & 7.19 & 5.78 \\
\hline 4 & & & 3.35 & 5.74 & 6.53 & 4.9 \\
\hline 5 & & & 5.42 & 7.31 & 6.02 & 4.23 \\
\hline 6 & & & 8.67 & 8.57 & 5.71 & 3.77 \\
\hline 1 & \multirow{8}{*}{0.44} & \multirow{8}{*}{0.5} & 0.72 & 3.12 & 13.11 & 20.52 \\
\hline 2 & & & 1.55 & 3.82 & 8.3 & 9.85 \\
\hline 3 & & & 2.42 & 5.24 & 7.01 & 6.5 \\
\hline 4 & & & 3.24 & 6.75 & 6.48 & 5.27 \\
\hline 5 & & & 4.57 & 8.67 & 6.07 & 4.46 \\
\hline 6 & & & 5.76 & 10.06 & 5.87 & 4.1 \\
\hline 7 & & & 7.51 & 11.26 & 5.73 & 3.86 \\
\hline 8 & & & 9.46 & 12.44 & 5.63 & 3.26 \\
\hline
\end{tabular}




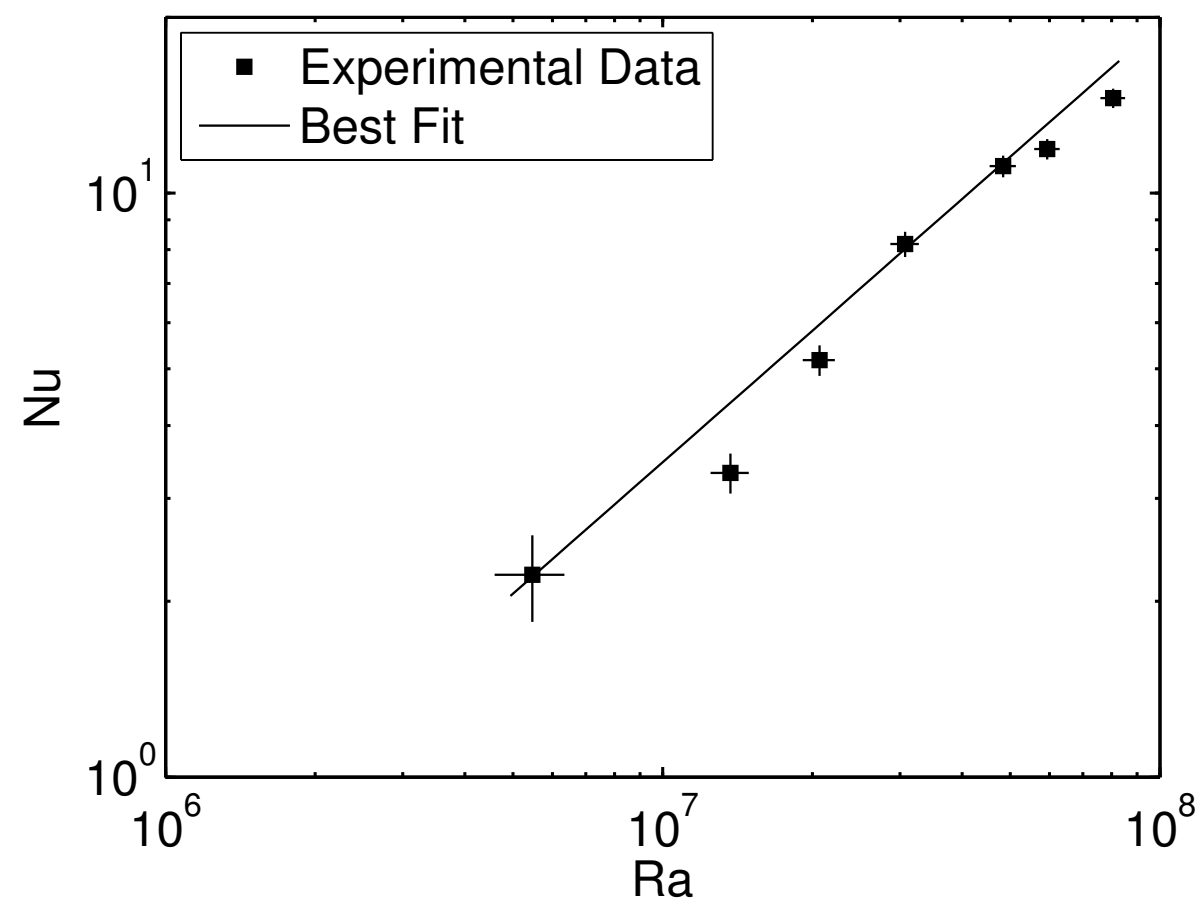

Figure 6.6: Nusselt-versus-Rayleigh number relation for $\eta=0.5, \delta=0.44$. Error bars indicate the total uncertainties.

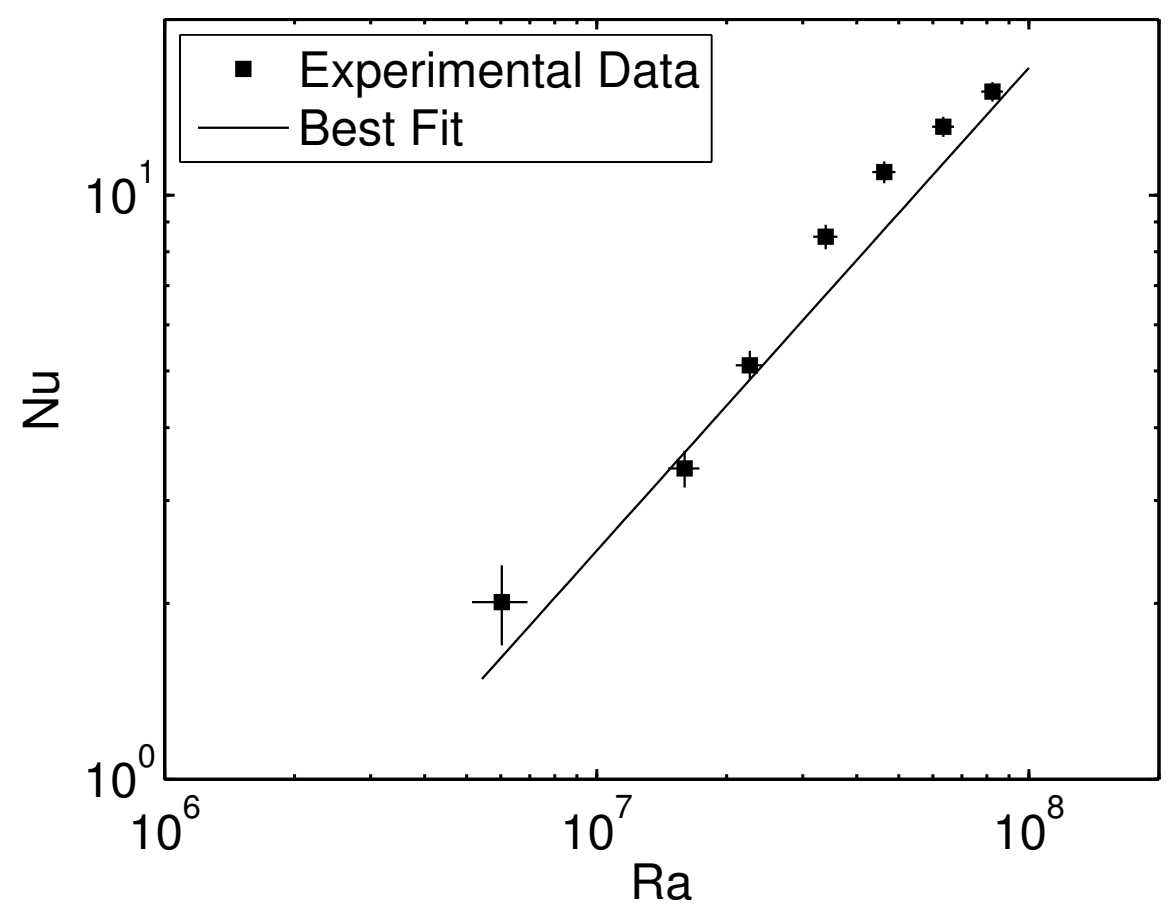

Figure 6.7: Nusselt-versus-Rayleigh number relation for $\eta=0.67, \delta=0.44$. Error bars indicate the total uncertainties. 


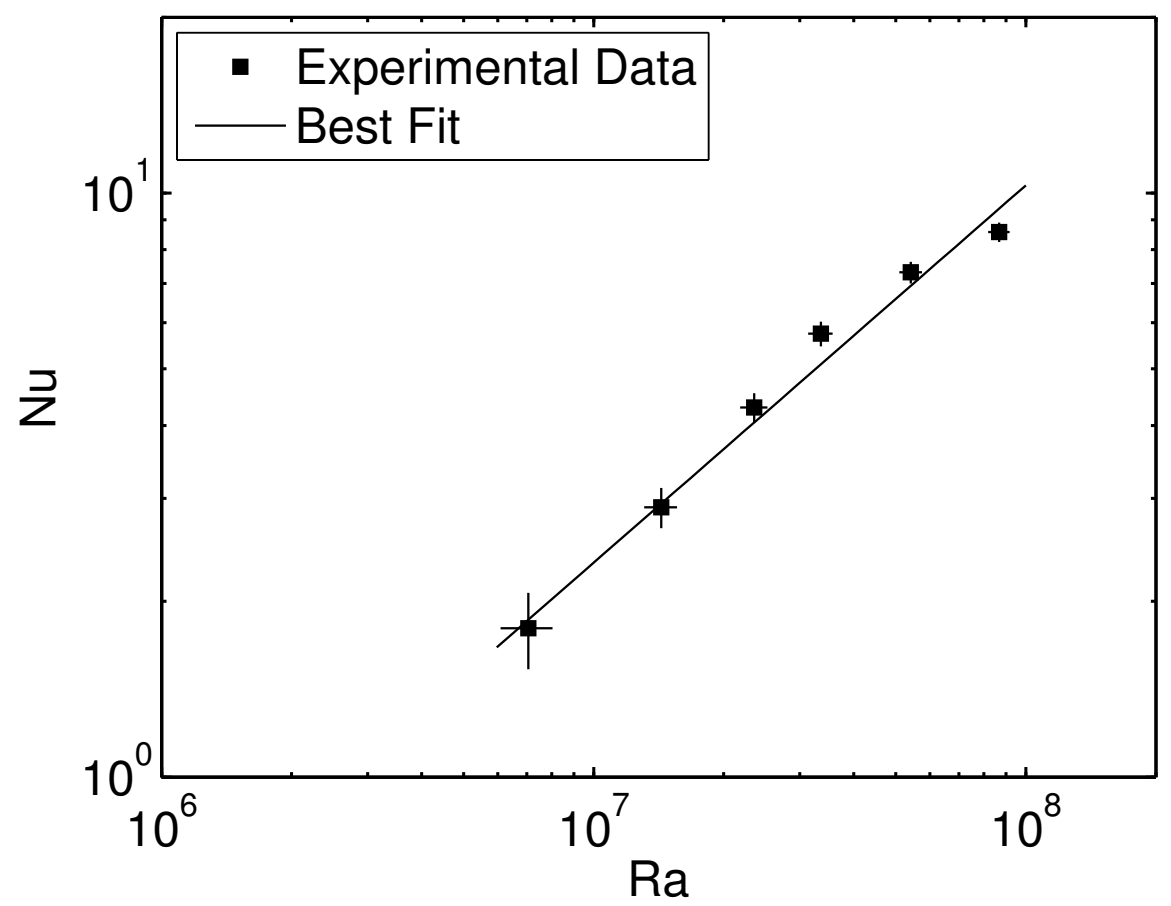

Figure 6.8: Nusselt-versus-Rayleigh number relation for $\eta=0.75, \delta=0.44$. Error bars indicate the total uncertainties.

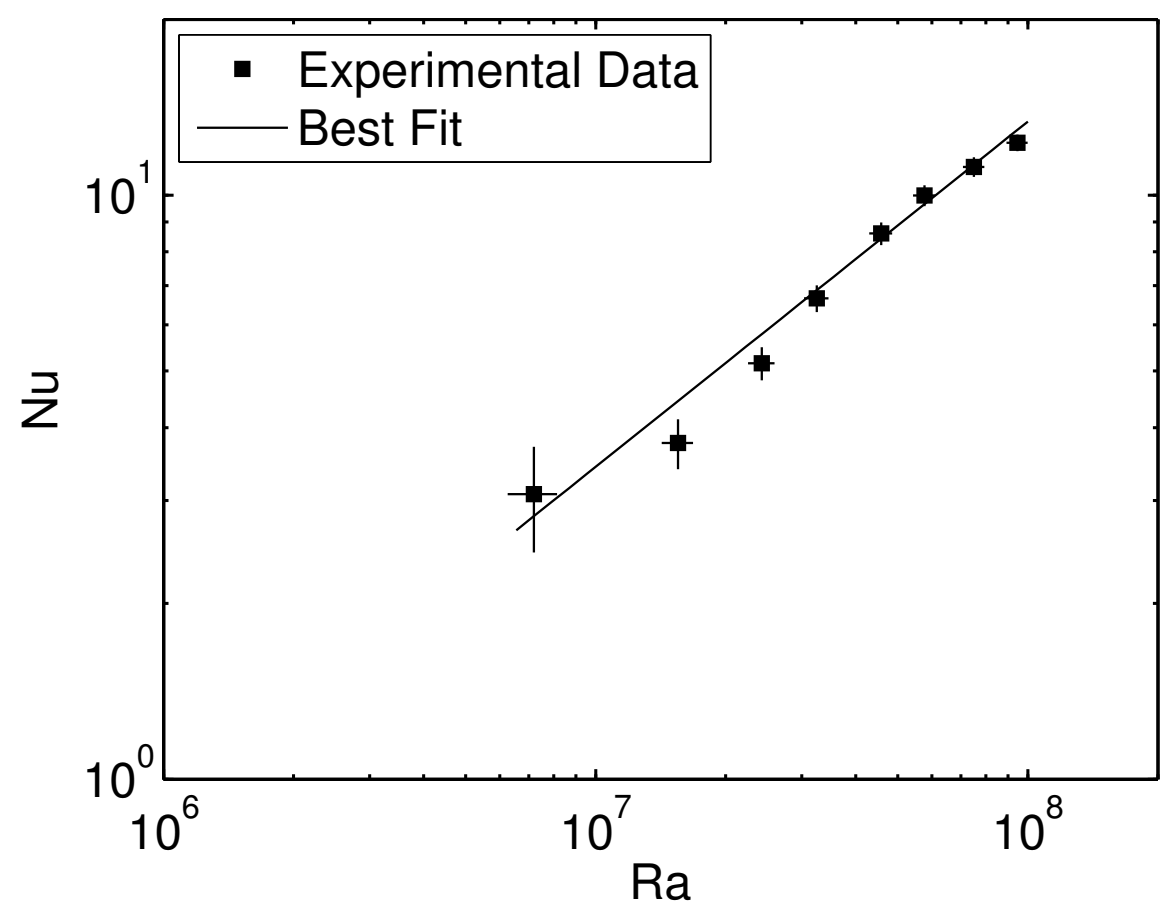

Figure 6.9: Nusselt-versus-Rayleigh number relation for $\eta=0.5, \delta=0.11$. Error bars indicate the total uncertainties. 


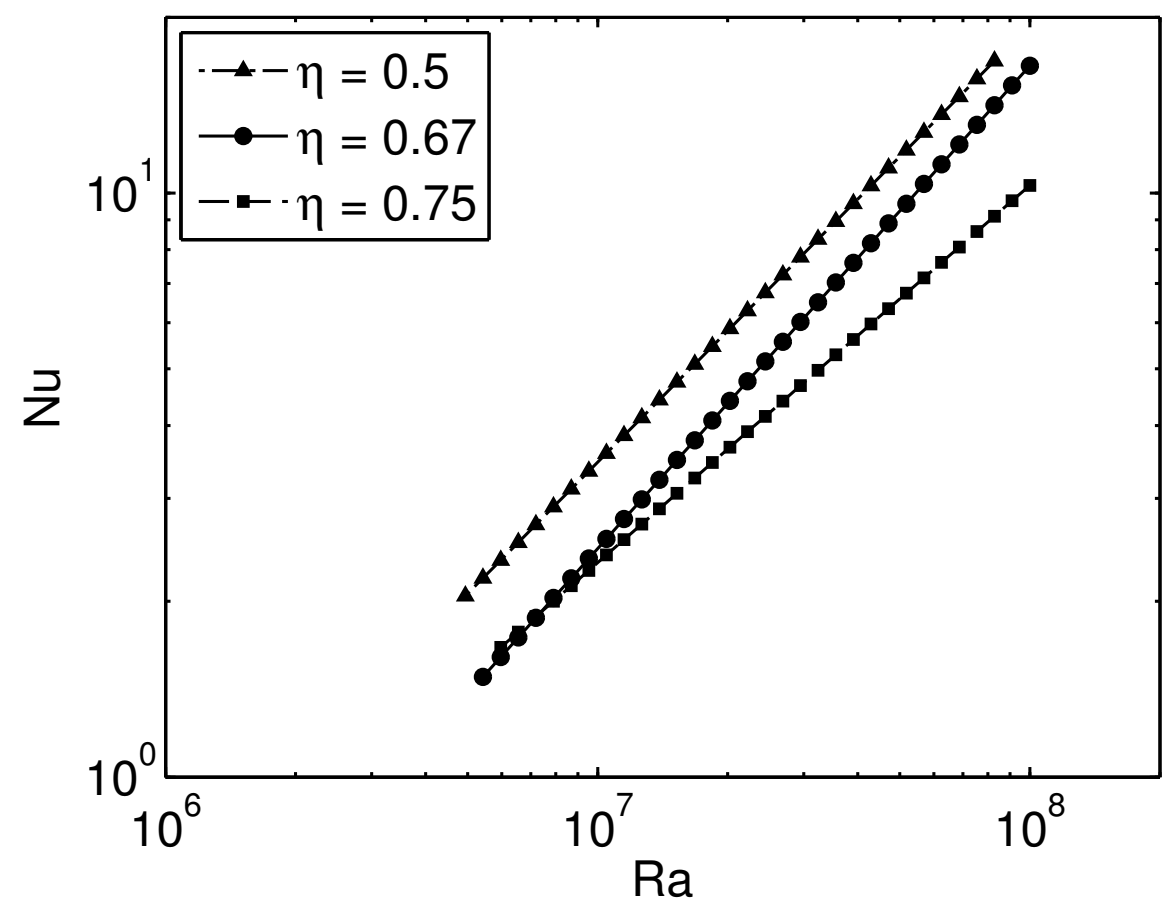

Figure 6.10: Effect of height ratio, $\eta$, on the overall Nusselt-versus-Rayleigh number relations $(\delta=0.5)$.

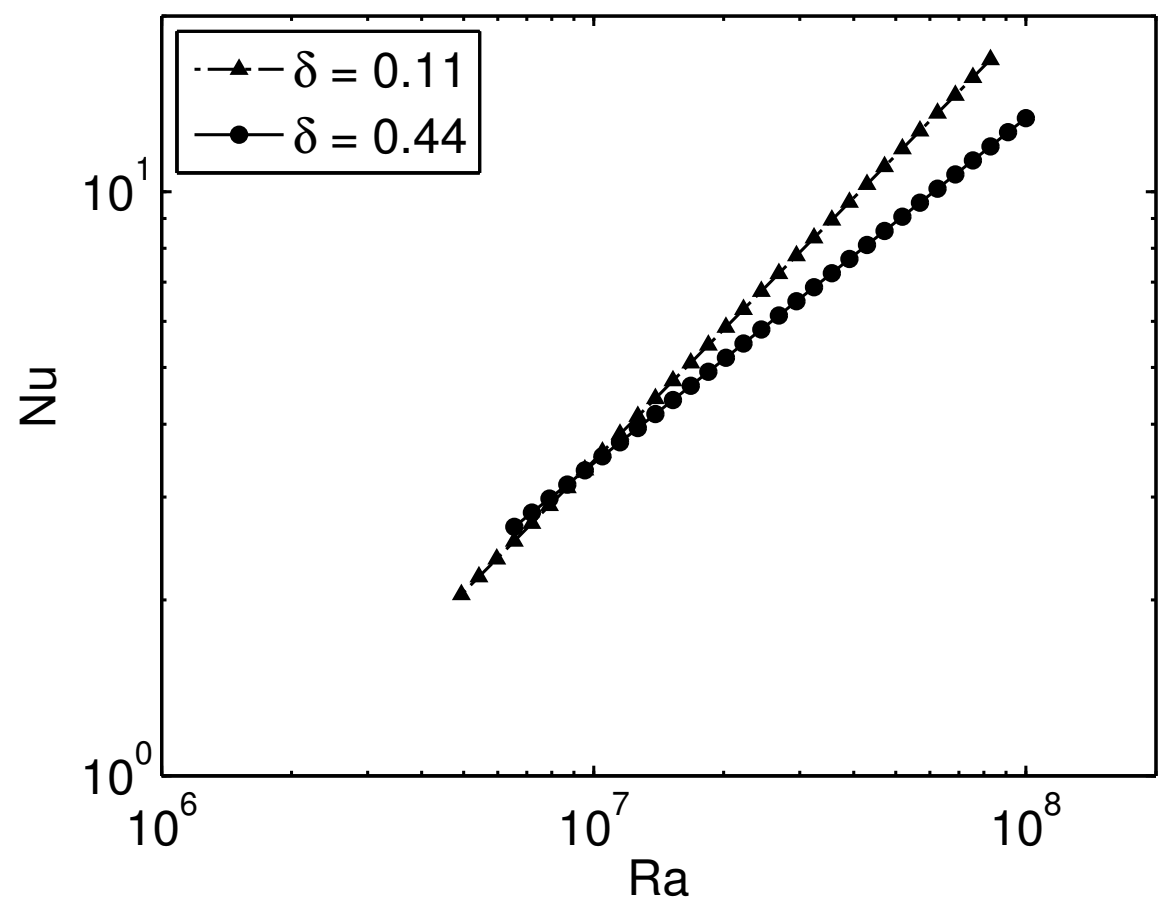

Figure 6.11: Effect of heater length ratio, $\delta$, on the overall Nusselt-versus-Rayleigh number relations $(\eta=0.5)$. 
Table 6.4: Curve fit parameters for all the experiments.

\begin{tabular}{ccc}
\hline Experimental parameters & Curve-fit equations & $R^{2}$ \\
\hline \hline$\delta=0.44, \eta=0.5$ & $N u=\left(2 \times 10^{-5}\right) \times R a^{0.7843}$ & 0.9779 \\
$\delta=0.44, \eta=0.67$ & $N u=\left(4 \times 10^{-6}\right) \times R a^{0.8277}$ & 0.9784 \\
$\delta=0.44, \eta=0.75$ & $N u=\left(7 \times 10^{-5}\right) \times R a^{0.6464}$ & 0.9833 \\
$\delta=0.11, \eta=0.5$ & $N u=\left(2.5 \times 10^{-4}\right) \times R a^{0.5911}$ & 0.9749 \\
\hline
\end{tabular}

numbers, while at lower values of Ra the two data sets overlap. As mentioned earlier, the most likely due the higher uncertainties in the overall heat transfer rates. It must also be borne in mind that this comparison is not a direct comparison of the actual Nusselt number data but rather of the best-fit curves. Inspection of the actual Nusselt and Rayleigh number data listed in Table 6.3 will show that the Nusselt number for $\delta=0.11$ is slightly higher than that for $\delta=0.11$ even at the lower Rayleigh numbers.

\subsubsection{Temperature profiles}

In the present set of experiments, temperature profiles are measured along the horizontal mid-plane of the convection cell in both the longitudinal (along the length of the heater) and the transverse directions (perpendicular to the heater direction). Figures 6.9 (a) shows the dimensionless temperature profiles for $\eta=0.67$ and $\delta=0.44$ at three different Rayleigh numbers. In order to illustrate the salient features of the temperature distribution, only a single set of measurements are discussed here. As can be seen, the transverse temperature profiles show a distinct plume like flow along the centerline of the convection cell above the heater surface. With increasing $R a$, the thermal plume becomes more pronounced. Even though the aspect ratio is 6 , a single plume is seen present only above the heater zone. This is in excellent agreement with the predictions of the numerical simulations. The most important aspect of the transverse temperature profile, however, is the vertical location of the thermal plume within the composite domain. Because the thermocouples are located at the horizontal mid-plane of the cavity and because $\eta=0.67$, the thermocouples are actually embedded within the porous layer below the fluid-porous layer interface. The presence of a plume-like flow inside the porous layer is direct evidence that convective motion is present inside the porous layer. This is direct confirmation of penetrative convection within the porous layer and is in agreement with the predictions of the numerical simulations. It also shows that the flow inside the porous layer is not conduction dominated. These will be discussed in further detail in the next chapter when the results of the numerical simulations are compared with the present experimental data. 


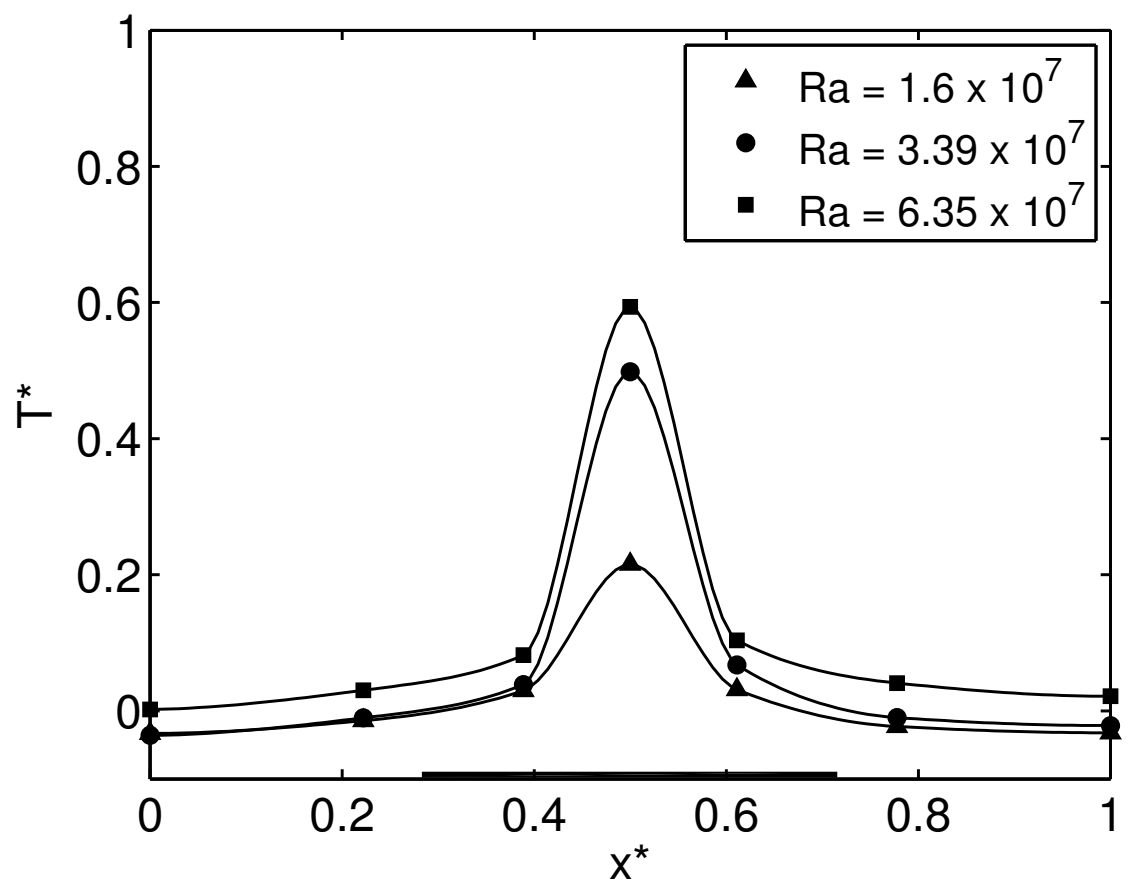

Figure 6.12: Non-dimensional temperature profiles along the length of the convection cell and along the horizontal mid-plane at three different $R a$ values. The position of the heater is also shown on the $x$-axis.

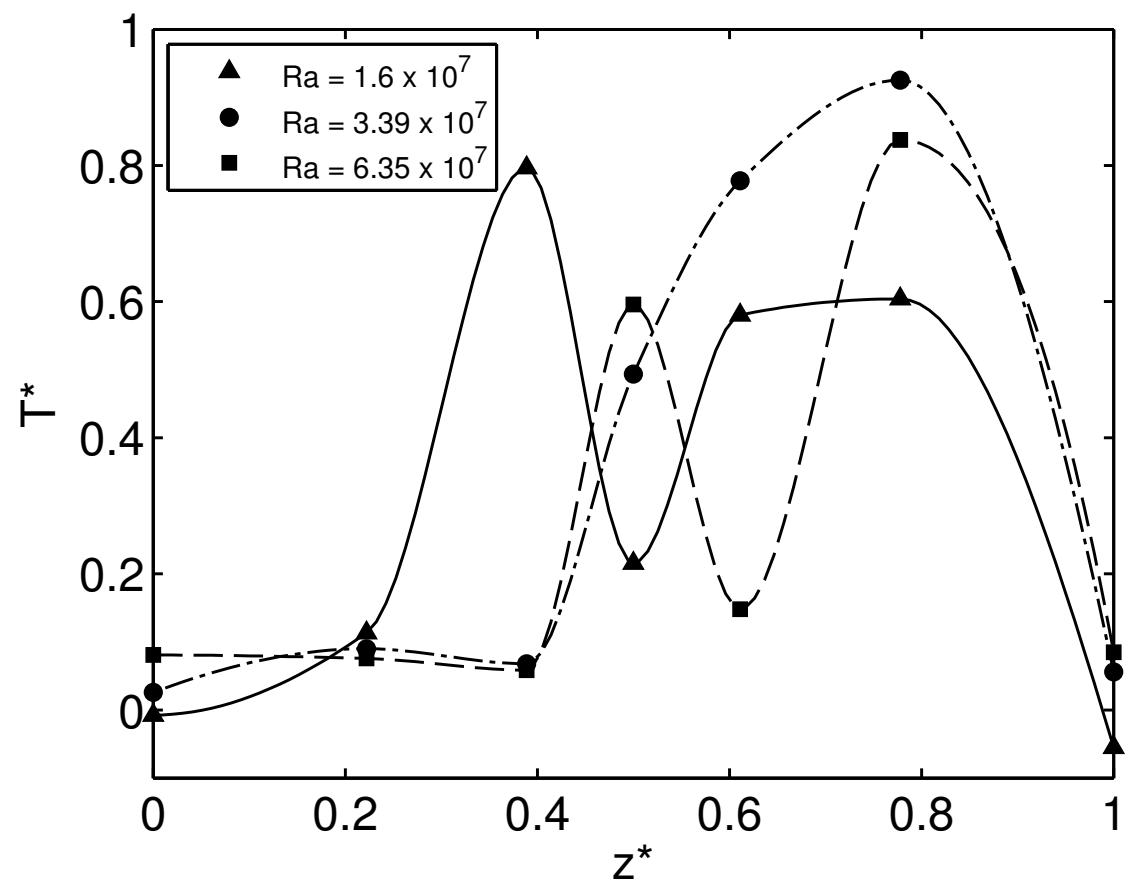

Figure 6.13: Non-dimensional temperature profiles along the depth of the convection cell and along the horizontal mid-plane at three different $R a$ values. 
The longitudinal temperature profiles shown in Figure 6.9(b), however, show a complex flow pattern. Interestingly, the temperature field changes with increasing Rayleigh number. For $R a=1.6 \times 10^{7}$, a single well developed thermal plume and another partially developed thermal plume are seen. At $R a=3.39 \times 10^{7}$, however, only a single plume can be seen. Further increase in $R a$ creates two well developed thermal plumes. This shows that the flow pattern changes and evolves with the Rayleigh number. It also reveals a flow structure that is highly asymmetric and three dimensional. These observations are in agreement with the experimental observations of Chen and Chen [14].

\subsection{Conclusions}

The experimental results from the present study have revealed several important features of convection heat transfer in fluid-superposed porous layers. These are:

- Heat transfer rates for all experiments are found to obey a power-law correlation, similar to observations made for different derivatives of the Rayleigh-Bénard problem.

- Results show that the overall heat transfer rates decrease with increase in the porousto-total height ratio $\eta$. This is in agreement with results of the numerical simulations as well as literature results on convection in fluid-superposed porous layers heated uniformly from below.

- The overall heat transfer rates are found to decrease with increase in the heater length ratio, $\delta$, as predicted by the numerical simulations.

- The temperature fields along the mid-plane of the cavity reveal that a plume like flow exists in the porous layer indicating the presence of penetrative convection and showing that heat transfer through the porous layer in not simply conduction-dominated.

- Finally it is seen that that flow patterns are highly asymmetric due to the threedimensional nature of the convective motion. 


\section{Chapter 7}

\section{Conclusions}

In this chapter, the final conclusions of the thesis are presented along with recommendations for future work. The chapter begins with a comparison of the numerical and experimental results. Thereafter, the discrepancy between the numerical predictions and empirical measurements is discussed in detail. Because the primary aim of the experiments is to validate the numerical model presented, the discussion focuses on the validity of the one-domain formulation. Numerical results available in the literature are compared and the discrepancies among different modeling approaches are commented upon. Thereafter, the importance of the present study in the context of the overall problem of convection in composite fluidporous systems is discussed. Finally, the limitations of the present study are pointed out and recommendations are made for future work in this field.

\subsection{Comparison of numerical and experimental results}

In order to accurately compare the results of the numerical and the experimental studies, a new set of simulations is performed using the independent controlling parameters of the experiments. These parameters are: $A=6, D a=4.04 \times 10^{-6}, \phi=0.36, \delta=$ $0.44, \lambda=1, F=0.52, \sigma=0.5, \eta=0.5,0.75$. Figures 7.1 and 7.2 , and Table 7.1 show a comparison of the numerical and experimental results. For both $\eta=0.5$ and $\eta=$ 0.75 , the difference between the numerical predictions and experimental measurements is significant in the Rayleigh number range where the two data sets overlap. This mismatch is more pronounced for $\eta=0.5$ that for $\eta=0.75$. However, in both cases, the percentage difference between the numerical and experimental data is $\geq 60 \%$. It can also be seen that the slope of the experimental data fit line is much higher than that of the numerical data. This mismatch causes the experimental fit line to meet the extrapolated numerical data fit line at higher Rayleigh numbers. This would seem to indicate that the numerical simulations agree with experimental results in the high Rayleigh number range $\left(\sim 4 \times 10^{7}\right)$. 


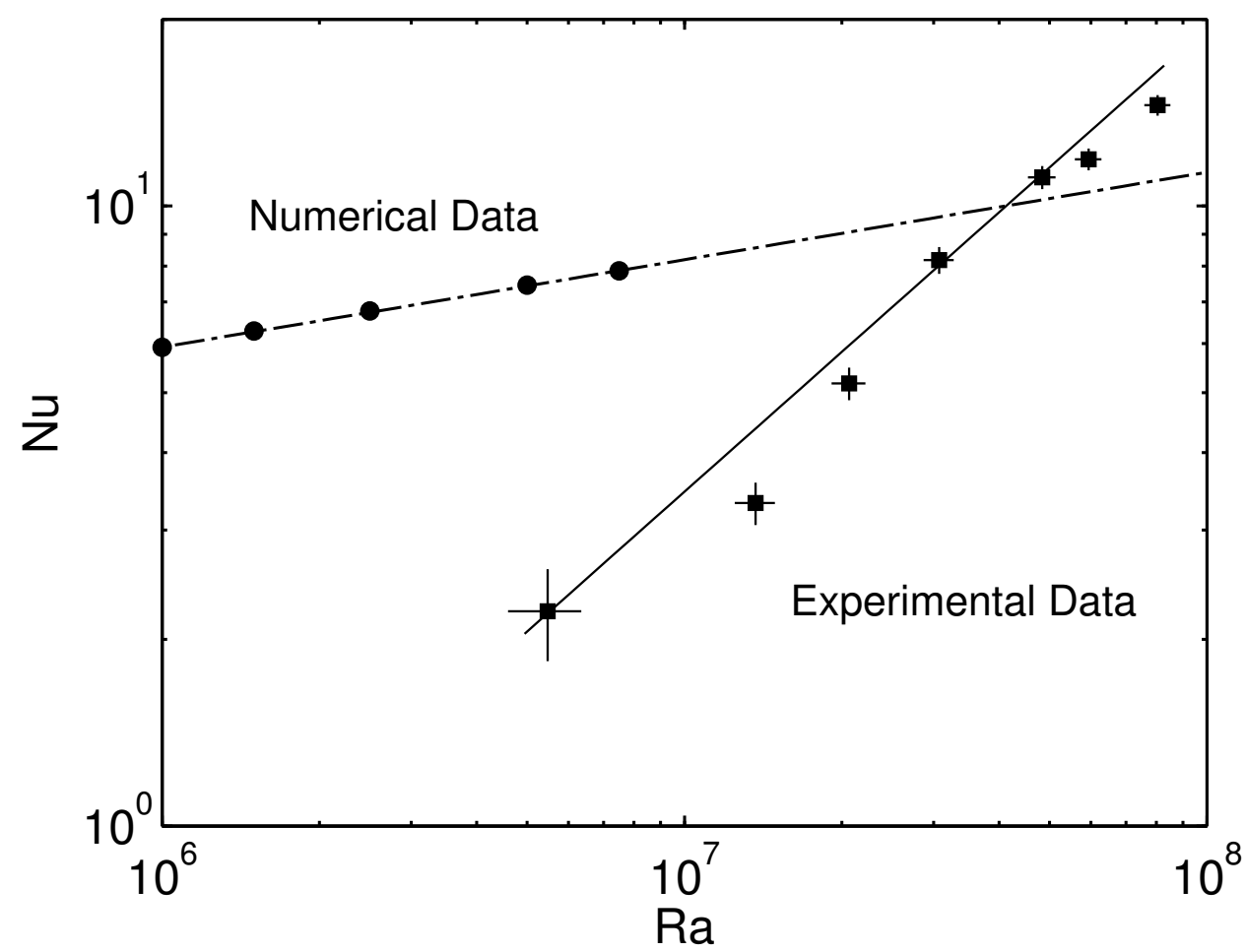

Figure 7.1: Comparison of numerical and experimental data for $\delta=0.44, \eta=0.5$.

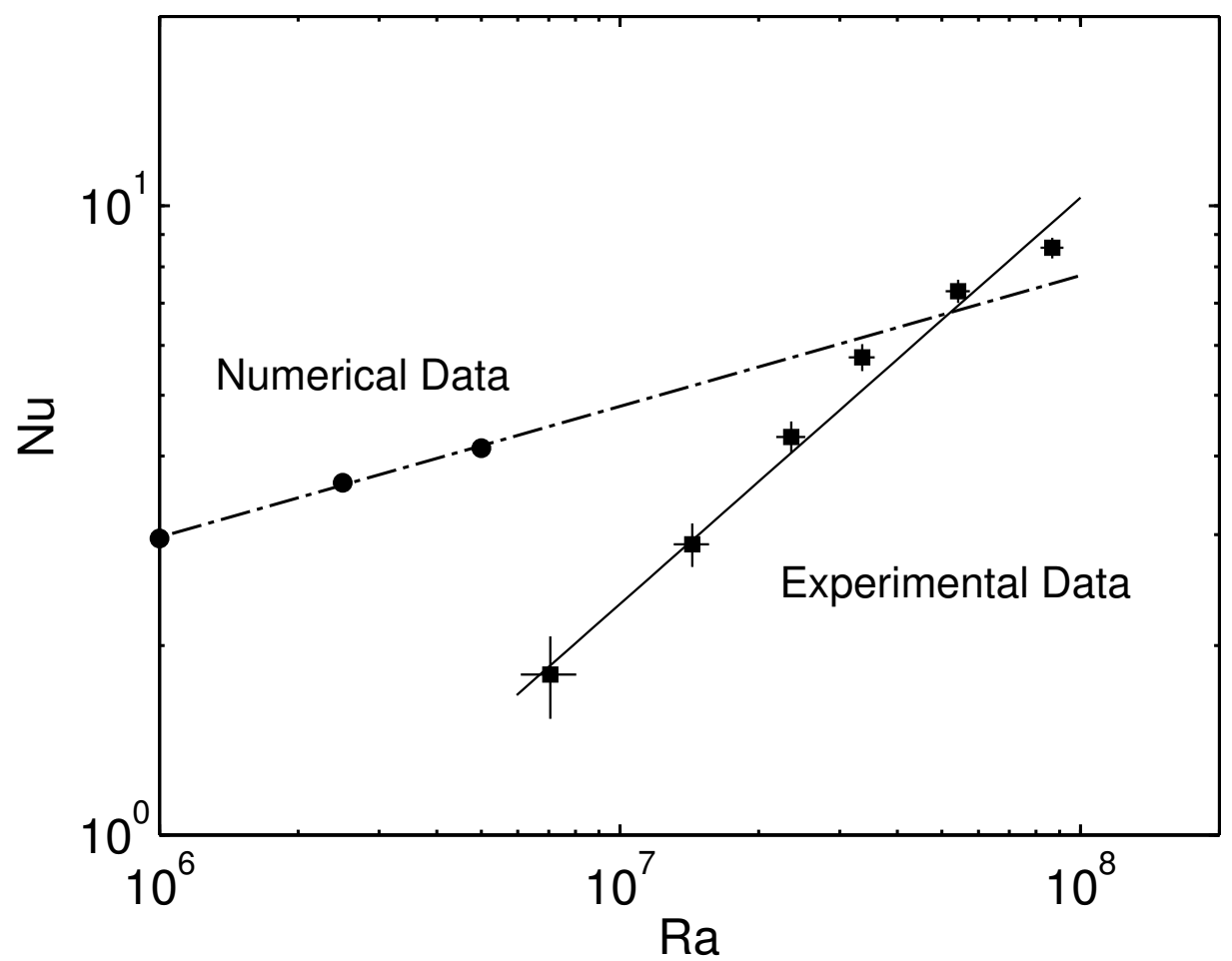

Figure 7.2: Comparison of numerical and experimental data for $\delta=0.44, \eta=0.75$. 
Table 7.1: Comparison of experimental and numerical results.

\begin{tabular}{|c|c|c|c|c|}
\hline Height Ratio, $\eta$ & $R a$ & $N u$ (Experimental) & $N u$ (Simulation) & $\%$ Error \\
\hline \hline \multirow{2}{*}{0.5} & $5.47 \times 10^{6}$ & 2.219 & 7.52 & 70.5 \\
& $7.5 \times 10^{6}$ & 2.79 & 7.86 & 64.5 \\
\hline 0.75 & $7.45 \times 10^{6}$ & 1.789 & 4.45 & 59.82 \\
\hline
\end{tabular}

However, because no simulations have been at such high Rayleigh numbers, this conclusion cannot be made with any degree of certainty. Also, the fundamentally different nature of the two curves indicates that there is a more fundamental underlying discrepancy between the numerical and experimental results. This discrepancy is also seen for the other two sets of experiments performed.

The observed discrepancy between the numerical and experimental results indicates that this mismatch cannot be simply attributed to factors such as the uncertainty in the experimental results or the grid convergence of the numerical solution. To confirm this fact, experiments for $\eta=0.5$ were carefully repeated twice. Also, simulations at a few Rayleigh numbers were run with finer grids and more stringent convergence criterion. However, no significant change in the overall nature of both the data sets was seen. Also, because both the computer code and the experimental setup were verified by comparison with literature results for well established test cases (Rayleigh-Bénard and Horton-Rogers-Lapwood problems), the observed difference cannot simply be attributed errors in post-processing of the data. It can therefore be concluded that the observed difference points to a discrepancy that is more fundamental in nature. To investigate the cause behind the observed differences, attention is focused on two fundamental aspects of the problem: i) problem definition and setup, and ii) mathematical formulation.

\subsubsection{Discrepancies due to differences in boundary conditions}

The first reason for the observed discrepancy between the numerical predictions and experimental results may be due to the differences in the boundary conditions for the two studies. In particular, attention needs to be focused on the boundary conditions along the lower surface. It may be recalled that the fundamental problem explored via the numerical simulations is that of two-dimensional natural convection in a fluid-superposed porous layer with a localized isothermal heat source at the base. The experiments on the other hand are conducted in a three-dimensional enclosure with a strip heater supplying a constant heat flux to the porous layer. The boundary conditions at the heater surface are, therefore, different for the two problems. This was noted in Chapter 5 while discussing the design of 
Table 7.2: Temperature distribution on the heater surface for $\eta=0.75, \delta=0.44$.

\begin{tabular}{ccccc}
\hline Run No. & $T_{H, \text { avg }}$ & S.D. (\%) & $T_{C, \text { avg }}$ & S.D. (\%) \\
\hline \hline 1 & 29.57 & 4.28 & 23.53 & 0.24 \\
2 & 33.98 & 9.99 & 22.83 & 0.38 \\
3 & 39.11 & 13.08 & 22.94 & 0.61 \\
4 & 43.84 & 13.66 & 23.23 & 0.90 \\
5 & 52.28 & 13.37 & 25.04 & 1.08 \\
6 & 61.79 & 14.3 & 24.65 & 1.61 \\
\hline
\end{tabular}

the experimental setup. The difference in boundary conditions can be better understood by comparing the temperature distributions at the heater and top surfaces for a single experiment $(\delta=0.44, \eta=0.5)$, as shown in Table 7.2. It can be seen that while a near isothermal condition exists at the top plate, the same is not true for the heater surface. For most runs, the percentage standard deviation (S.D.) of the heater surface temperature is greater than $10 \%$. The large variation in temperature across the heater, which is in part responsible for the high uncertainties in $N u$ and $R a$, indicates that a direct comparison to the numerical data is not appropriate.

Another point of discrepancy lies in the boundary conditions at the base outside the heater area. The formulation for the numerical solution assumes that base area outside the heated region is adiabatic. This condition is however not replicated in the experimental setup. As seen in the previous chapter, a numerical solution of the conduction problem in the base plate shows that there is a small amount of heat flow from the area outside the strip heater into the porous layer. Although this heat flow is a very small fraction of the total energy supplied by the heaters, it nevertheless causes a part of the area outside the strip heater to be non-adiabatic.

The differences in the boundary conditions mentioned above indicate that a certain amount of difference between the numerical and experimental results can be expected. However, it is worthwhile to investigate whether all of the observed discrepancy occurs solely because of this reason. As there are no published studies on convection in fluidsuperposed porous layers with a constant heat flux boundary condition at the base, a direct estimation of the effect of the boundary conditions on the Nusselt and Rayleigh numbers is not possible. There are, however, several studies on natural convection in porous layers with localized isothermal and constant flux heat sources at the base. Because the porous layer convection problem is a special case of the present problem for $\eta=1$, results from 
these studies can be used to understand the effect of the boundary conditions on the overall heat transfer coefficients.

A comparison between the numerical results of Prasad and Kulacki [58] with the numerical and experimental results of Rajen and Kulacki [60] is chosen to investigate the effects of the boundary conditions. The simulations of Prasad and Kulacki are performed with the following parameters: $A=10, \delta=0.5$, centrally positioned isothermal heater. The numerical and experimental results of Rajen and Kulacki use the following parameters: $A=9.6, \delta=0.5$, centrally positioned constant flux heater. Except for the boundary condition at the heater surface, the two studies have near-identical problem formulations and boundary conditions and are ideally suited for comparison. It must be noted that Prasad and Kulacki define the Rayleigh number, $R a_{\mathrm{m}}$, based on the temperature difference across the heated and cooled surfaces. On the other hand, Rajen and Kulacki use a flux based definition for the Rayleigh number $R a_{\mathrm{m}}^{*}$. The two Rayleigh numbers are related as,

$$
R a_{\mathrm{m}}^{*}=R a_{\mathrm{m}} \times N u_{\mathrm{m}},
$$

where $N u_{\mathrm{m}}$ is the Nusselt number based on the stagnant conductivity of the porous layer. Using equation (7.1), the $N u_{\mathrm{m}}$-versus- $R a_{\mathrm{m}}^{*}$ data of Rajen and Kulacki [58] is converted to $N u_{\mathrm{m}}$-versus- $R a_{\mathrm{m}}$ data for comparison. This is similar to the experimental data reduction procedure adopted in the present study where the temperature based Rayleigh number, $R a$, is used for a constant flux heater.

Comparison of the data for the above studies is shown in Figure 7.3. The differences in the two sets of data are readily apparent. This confirms the fact that heat transfer coefficients change with a change in the boundary conditions. However, several interesting facts can be observed from Figure 7.3. First, even though the two sets of data are different, the $N u_{\mathrm{m}}$-versus- $R a_{\mathrm{m}}$ curves have almost identical overall character although the slope of the curve for the constant heat flux data is higher. In addition, for a given $R a_{\mathrm{m}}$, the value of $N u_{\mathrm{m}}$ is higher for the constant heat flux boundary condition data. If these observations are extrapolated to the present problem, one would expect to see: a) identical characters for the numerical and experimental $N u$-versus- $R a$ curves, and b) experimental data overpredicting $N u$ over the entire range of $R a$. It can be immediately seen that none of these characteristics are observed for the present data. Based on this it can be concluded the observed discrepancy between the numerical and experimental results of the present study cannot be attributed to the differences in boundary conditions for the two studies. The only effect that the boundary conditions may have is to make the $N u$-versus- $R a$ curve for the experimental data to be steeper than the curve for the numerical data. 


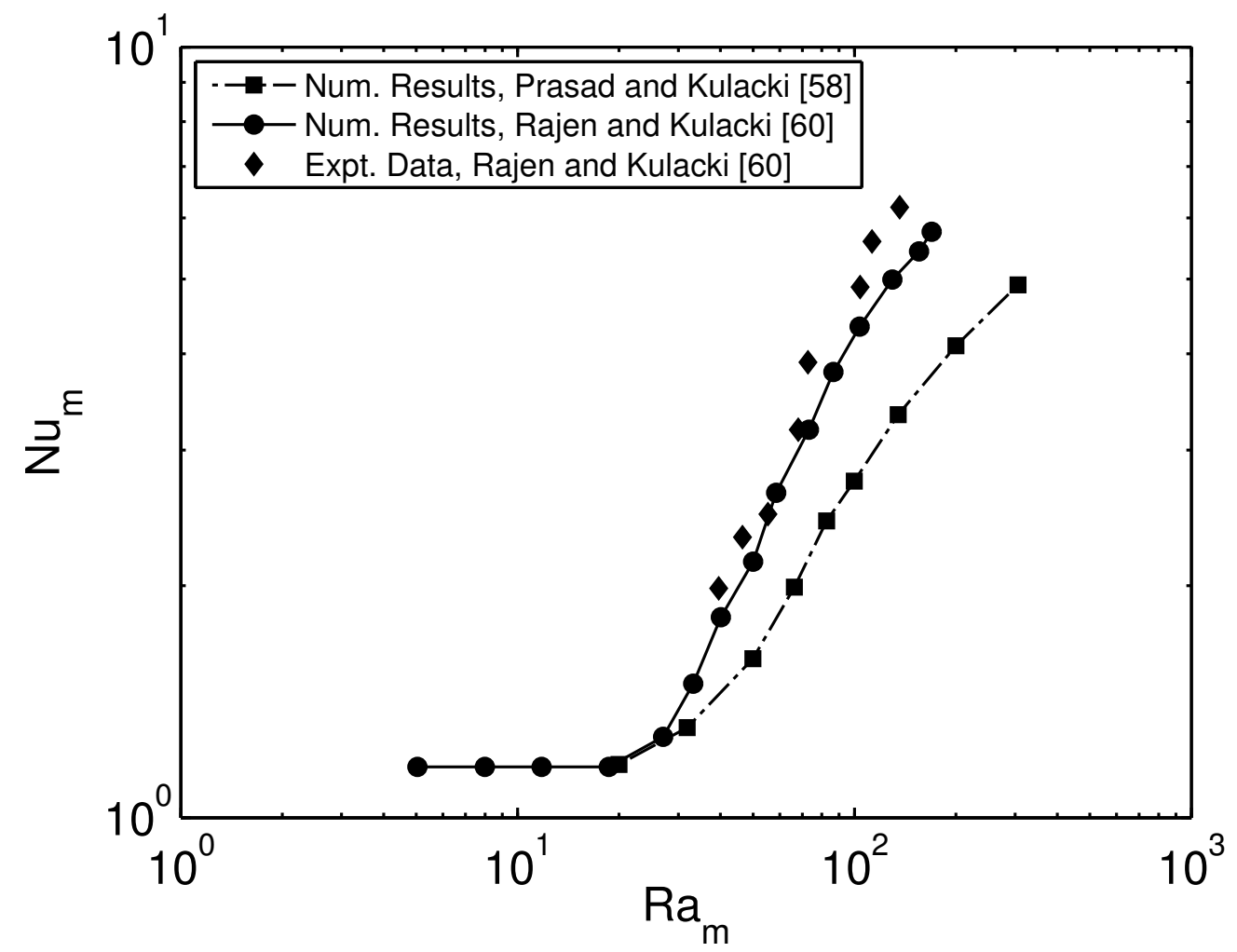

Figure 7.3: Comparison of the numerical and experimental results of Prasad and Kulacki [58], and Rajen and Kulacki [60].

\subsubsection{Effect of the mathematical model}

The other possible source of discrepancy between the simulation and experimental data may lie in the mathematical formulation of the problem for the numerical solution. In particular, attention must be focused on the one-domain formulation that is adopted in the present study. It may well be that the limitations of this particular formulation are responsible for the observed lack of agreement with the experimental results. If this is the case, it is vital that these limitations be identified so that suitable corrections can be made.

In order to further explore this issue, it is important to first understand the validity and accuracy of the one-domain formulation. An extensive review of the literature reveals that there has been no thorough experimental validation of the one-domain formulation for the present problem. The only reported comparison of simulations using this formulation to experimental data has been reported by Kim and Choi [23]. The authors determined the critical wave number for different height ratios and found good agreement with the experimental data of Chen and Chen [10]. They also determined the Nusselt numbers for Rayleigh number up to two times the critical value at $\eta=0.91$ and found good agreement with Chen and Chen's [10] results. However, they did not validate their numerical solution at Rayleigh number much higher than the critical point. Because of this, it is quite possible 


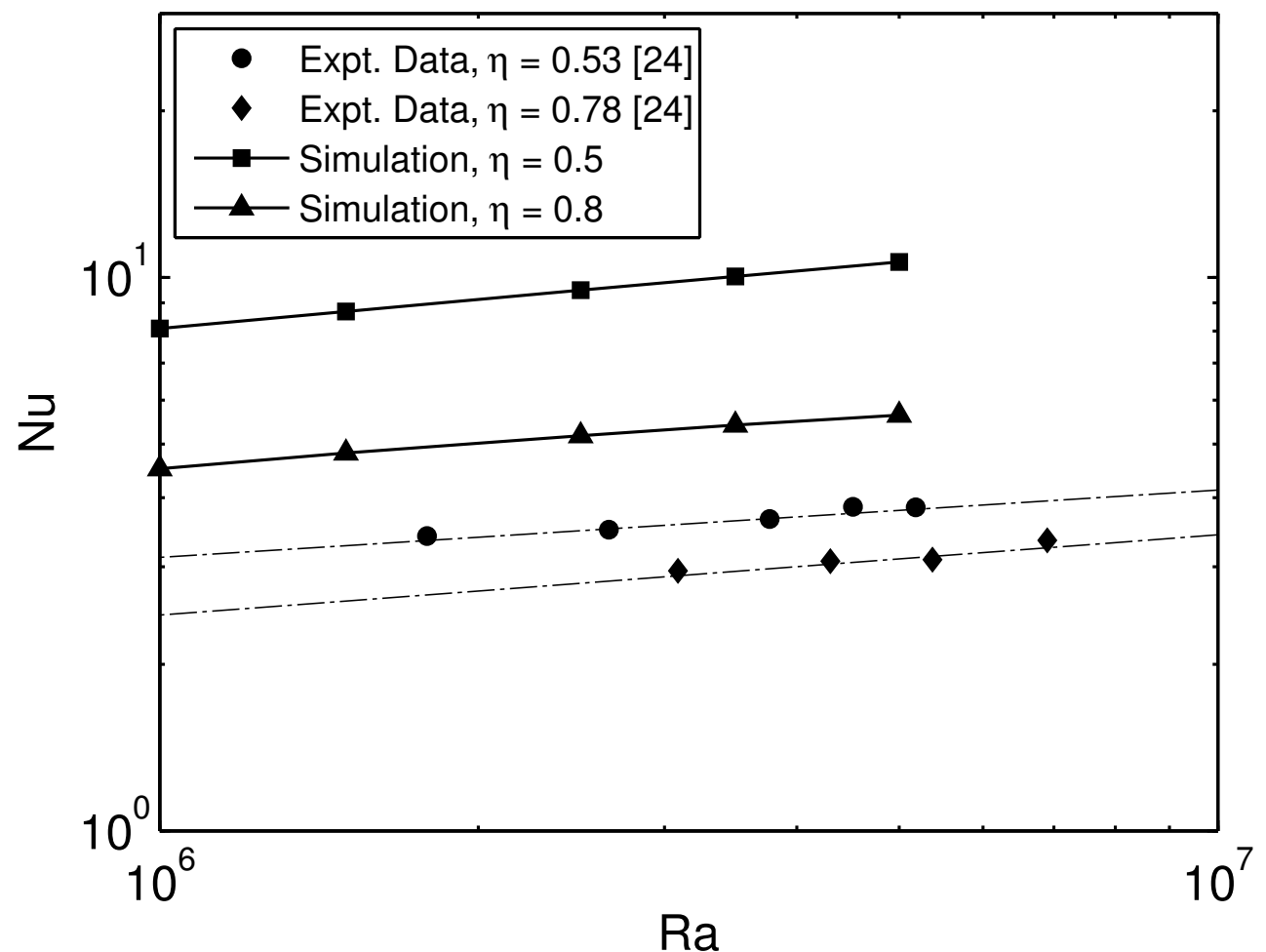

Figure 7.4: Comparison of simulations results for $\delta=1$ and $\eta=0.5,0.8$ with the experimental results of Prasad and Tian [24].

that the model may have some deficiencies, especially in the high Rayleigh number range where its validity is yet to be demonstrated. To explore this possibility, simulations are performed with the present code for the case of convection with a uniformly heated base and the results are compared with the experimental results of Prasad and Tian [24]. The parameters used in the simulations are: $A=2, D a=3.71 \times 10^{-6}, \phi=0.396, \lambda=$ 6.8, $\operatorname{Pr}=8835, F=0.56, \sigma=0.7, \eta=0.5,0.8$. These parameters are suitable for a randomly packed layer of $6 \mathrm{~mm}$ DIA glass beads saturated with Dow Corning 200 Silicone oil and are selected to facilitate direct comparison with Prasad and Tian's [24] data.

Comparison of the results of the simulations with the data of Prasad and Tian [24] is shown in Figure 7.4 and Table 7.3. The marked discrepancy between the two sets of data is clearly apparent. Although Prasad and Tian [24] do not provide information on the uncertainties in their measurements, it can be safely said that such a large disagreement cannot be simply due to the uncertainties. From Table 7.3 it can be seen that the difference between the simulation and experimental data is higher for $\eta=0.5$ than for $\eta=0.8$. This is identical to what is observed for the present experimental measurements. It can therefore be said that the discrepancy between the theoretical and experimental results is highest at intermediate height ratios $(\eta \sim 0.5)$. The likely reason for this is that the simulation results 
Table 7.3: Comparison of numerical simulations with data of Prasad and Tian [24].

\begin{tabular}{|c|ccc|ccc|}
\cline { 2 - 7 } \multicolumn{1}{c|}{} & \multicolumn{3}{c|}{$\eta=0.53$} & \multicolumn{3}{c|}{$\eta=0.78$} \\
\hline$R a$ & $N u$ (Sim.) & $N u$ (Expt.) & $\%$ Diff. & $N u$ (Sim.) & $N u$ (Expt.) & $\%$ Diff. \\
\hline \hline $1 \times 10^{6}$ & 8.08 & 3.11 & 61.42 & 4.51 & 2.45 & 45.60 \\
$1.5 \times 10^{6}$ & 8.67 & 3.27 & 62.25 & 4.81 & 2.60 & 45.95 \\
$2.5 \times 10^{6}$ & 9.48 & 3.48 & 63.21 & 5.17 & 2.80 & 45.86 \\
$3.5 \times 10^{6}$ & 10.04 & 3.63 & 63.81 & 5.41 & 2.94 & 45.62 \\
$5 \times 10^{6}$ & 10.66 & 3.79 & 64.42 & 5.64 & 3.09 & 45.06 \\
\hline
\end{tabular}

agree with experimental data for the special case of $\eta=1$. As such, the gap between the numerical and experimental results shrink as this limiting value is approached.

The comparison with the data of Prasad and Tian [24] seems to indicate that the present implementation of the one-domain model gives physically unrealistic data in the high Rayleigh number range. It may be recalled from Chapter 4 that simulation data obtained with the present code was compared to the numerical results of Poulikakos [21] and excellent agreement was obtained. However, because Poulikakos [21] too used a onedomain formulation it can be concluded that the problem may not lie in the implementation of the model in the computer code. To get a better idea of the influence of the effect of the modeling on the heat transfer results, results from all numerical studies available in the literature are compared with the present simulation results for $\delta=1$. This is shown in Figure 7.5. The parameters used in each of the compared studies are listed in Table 7.4. It can be seen that the simulation parameters used in all the studies are more or less identical. The only parameters that differ somewhat among all studies are the aspect ratio, $A$, and the Darcy number, Da. However, as seen in Chapter 4, these parameters do not significantly affect the overall heat transfer rates. As such, the comparison can give a good idea of the effect of the modeling approach. It must be noted that three difference modeling approaches are compared here: i) the two-domain approach which uses Darcy's law in the porous layer and the Beavers-Joseph slip boundary condition [64] at the interface [20], ii) a two-domain formulation which uses Brinkman's extension to Darcy's law in the porous layer and the continuity of shear stress and tangential velocity at the interface [22], and iii) a one-domain formulation which does not involve any explicit specification of the interfacial boundary conditions $[21,23]$.

A quick glance at Figure 7.5 shows how the particular modeling approach drastically affects the overall $N u$-versus- $R a$ relation. In particular, it can be seen how different results 
Table 7.4: Parameters used in the different numerical studies compared.

\begin{tabular}{cccccc}
\hline Parameter & $\begin{array}{c}\text { Poulikakos } \\
\text { et al. }[20]\end{array}$ & $\begin{array}{c}\text { Poulikakos } \\
{[21]}\end{array}$ & $\begin{array}{c}\text { Chen and } \\
\text { Chen }[22]\end{array}$ & $\begin{array}{c}\text { Kim and } \\
\text { Choi }[23]\end{array}$ & $\begin{array}{c}\text { Present } \\
\text { Study }\end{array}$ \\
\hline \hline A & 2 & 2 & Infinite & 5 & 2 \\
$\mathrm{Da}$ & $10^{-6}$ & $10^{-4}$ & $2.2 \times 10^{-6}$ & $2.2 \times 10^{-6}$ & $10^{-6}$ \\
$\eta$ & 0.5 & 0.5 & 0.5 & 0.5 & 0.5 \\
$\operatorname{Pr}$ & 7 & 7 & 6.26 & 6.26 & 7 \\
$\lambda$ & 1 & 1 & 1.62 & 1.62 & 1 \\
\hline
\end{tabular}

are obtained using the one-domain formulation. While, the present results show excellent agreement with the results of Poulikakos [21], they differ significantly from those of Kim and Choi [23] although all three studies use the one-domain formulation. This indicates that the limitations of the one-domain model may lie not in the mathematical formulation of the model but rather in its implementation for the numerical solution.

The most conclusive evidence for this hypothesis can be found in a series of recent publications by Hirata and co-workers [17-19]. This fact was pointed out earlier in Chapter 2 but its significance can be best understood in the present context. To briefly recall the earlier discussion, Hirata et al. [17] first published a study in which they investigated the effect of the mathematical formulation of the problem on the prediction of the stability criterion for the onset of convection. In particular, they compared three different modeling approaches: The results of Hirata et al. [17] show that the modeling approach adopted has a profound effect on the prediction the stability criterion. They found that while both the two-domain formulations give almost identical marginal stability curves, the curves obtained using the one-domain formulation are markedly different. The stability curves for the one-domain formulation do not exhibit the signature bi-modal character that is unique to the problem of convection in fluid-superposed porous layers. Also the critical Rayleigh numbers predicted by the one-domain formulation differ from those predicted by the twodomain approaches by 30-40 \% at different height ratios. This is contrast to the findings of Zhao and Chen [15] who too studied the stability problem using the one-domain formulation and found that the stability curves show the expected bi-modal character. These findings further point to the fact that the results obtained using the one-domain formulation strongly depend on the particular solution technique adopted.

To further investigate the source of this discrepancy, Hirata et al. [19] re-examined the same problem with a different approach to the numerical treatment of the interfacial conditions. In their problem formulation, the authors incorporated the hypothesis proposed by 


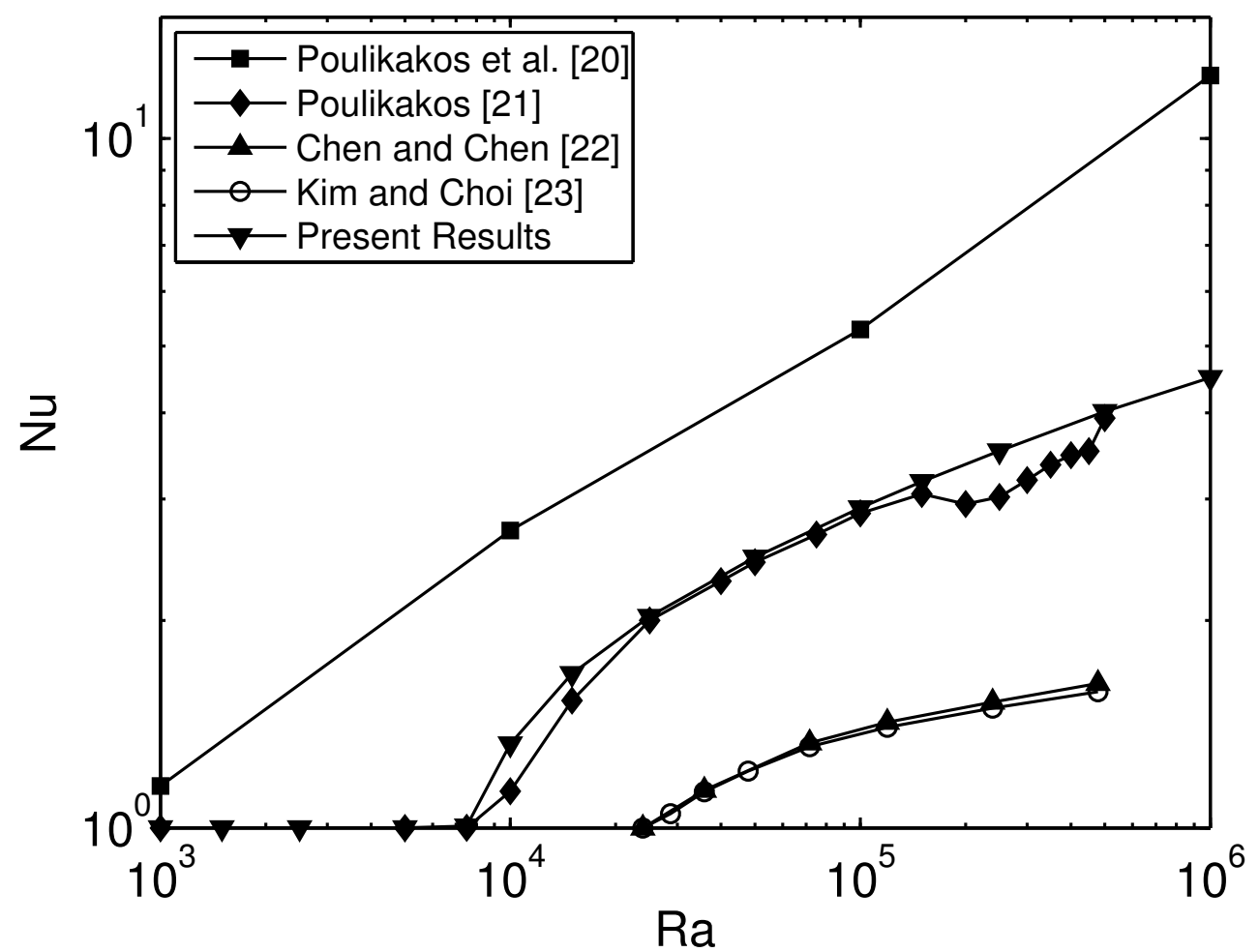

Figure 7.5: Comparison of present results for $\delta=1$ with all published numerical studies.

Kataoka [71] that the average properties of the porous medium such as the porosity, permeability, and effective diffusivity are Heaviside step functions and hence their differentiation must be considered in the meaning of distributions. Using this approach they found that the marginal stability curves for the one domain and two domain approaches were almost identical and showed almost the same bimodal behavior irrespective of the depth ratio. Based on their results the authors concluded that the one- and two-domain approaches are identical provided that the one domain approach is properly interpreted mathematically, i.e., in the meaning of distributions. Based on this evidence, it can be concluded that the limitation of the one-domain formulation lies in its implementation in a numerical code. This conclusion is further supported by the fact the mathematical formulation of the onedomain model is identical to that of the two-domain model which assumes the continuity of velocity, normal stress, shear stress, heat flux and temperature at the fluid-porous layer interface. The model is different only in the sense that it does not involve the explicit specification of the interfacial boundary conditions.

It can therefore be concluded special care must be taken while numerically implementing the one-domain formulation. In particular, the discretization equations for the interfacial control volumes must be treated separately so that the differentiations of the Heaviside step functions are correctly performed. It must be mentioned here that approach 
adopted by Hirata et al. [19] must not be considered as an integral part of the one-domain model. Other reported results obtained using the one-domain model obtain good agreement with experimental results without using specific mathematical constructs [71]. Also, their approach has not been verified by any subsequent study. Of special note is the fact that their study has been restricted to the determination of the stability criterion. The extension of this approach to the high Rayleigh number domain of the problem is still lacking. Most importantly though, all existing studies devoted to the stability problem still rely on a single set of experiments, performed by Chen and Chen [10], for validation. These experiments were performed over twenty years ago and have not been replicated by other authors. Therefore, at this point it cannot be conclusively said that the mathematical approach proposed by Hirata et al. [19] can resolve the conundrum regarding the most accurate mathematical model for the fluid-porous layer model. However it is an avenue that must be explored.

A question that must be answered at this point is why the one-domain model was chosen considering the confusion that exists regarding its implementation. The reasoning behind using the model is two-fold. First, the model is much simpler to use and implement than other modeling approaches as a single set of governing equations can be used for the entire domain and discretization equations for the boundary conditions do not have to be separately derived. Secondly, from a physical perspective, the one-domain model is more generic. It may be recalled that the crux of the model is that the porosity of the porous layer acts as a switching parameter that allows the appropriate form of the governing equations to be selected based on whether the solution domain lies in the fluid or porous layers. In the present study the porosity is a binary parameter and can only assume the values zero and unity. However, there is no restriction on the values that it can take. This allows problems involving non-homogenous, fissured, and layered porous media to be handled easily by simply assigning spatial or functional values to the porosity. This property makes the one-domain formulation very powerful. When coupled with its ease of programming and implementation, it can be easily seen why this technique is so attractive. That is the reason why so much research has been recently devoted to reconcile this formulation with existing two-domain models.

\subsection{Contributions of the present study}

The present study has been undertaken to study the phenomenon of natural convection heat transfer in fluid-superposed porous layers heated locally from below. This particular topic was primarily selected as it had not been explored previously. The objective at the beginning of the study was to make a fundamental contribution to the existing literature so that a more complete picture of the dual porous-fluid could be obtained. The 
Table 7.5: Parameters used in the different experimental studies compared.

\begin{tabular}{ccccc}
\hline Study & $A$ & $D a$ & $P r$ & $\lambda$ \\
\hline \hline Prasad et al. [24] & 2 & $3.27 \times 10^{-6}$ & 8835 & 6.81 \\
Prasad et al. [25] & 2 & $1.3 \times 10^{-5}$ & 578 & 1.19 \\
Prasad et al. [26] & 2 & $8.2 \times 10^{-5}$ & 8835 & 1 \\
Chen and Chen [10] & 6 & $2.2 \times 10^{-6}$ & 6.26 & 1.62 \\
Steven [68] & 0.5 & $3.1 \times 10^{-6}$ & 6.26 & 1 \\
Present Study & 6 & $4.4 \times 10^{-6}$ & 6.26 & 1 \\
\hline
\end{tabular}

combination of numerical and experimental methods has led to the following contributions:

- This is the first study to report numerical and experimental results on convection in fluid-superposed porous layers.

- This is the first study to report experiments with a constant heat flux boundary condition.

- This study represents an important step in trying to validate numerical predictions in the high Rayleigh number range through carefully designed experiments. Such validations of theoretical studies are virtually absent in the literature owing to the difficulty in designing experiments which can measure heat transfer rates in the Rayleigh number range that is accessible to simulations.

- The numerical simulations have highlighted some of the challenges that arise when implementing the one-domain formulation.

The contributions of the present study can be better understood by considering the results in the overall context of the problem of convection in fluid-superposed porous layers. As can be gathered from the discussion in the preceding section, the vast differences in the predictions of different numerical studies highlight some of the challenges that exist in accurately modeling this problem. Although numerical solutions of this problem were first published over twenty five years ago, no accepted set of results are yet available for this problem even for the case of a uniformly heated base. This is especially true for the high Rayleigh number regime where lack of any experimental validation has made impossible to verify the accuracy of any of the modeling approaches proposed.

Similarly experimental studies have focused on studying very small sections of the entire convection heat transfer regime. This is best illustrated in Figure 7.6 which shows 


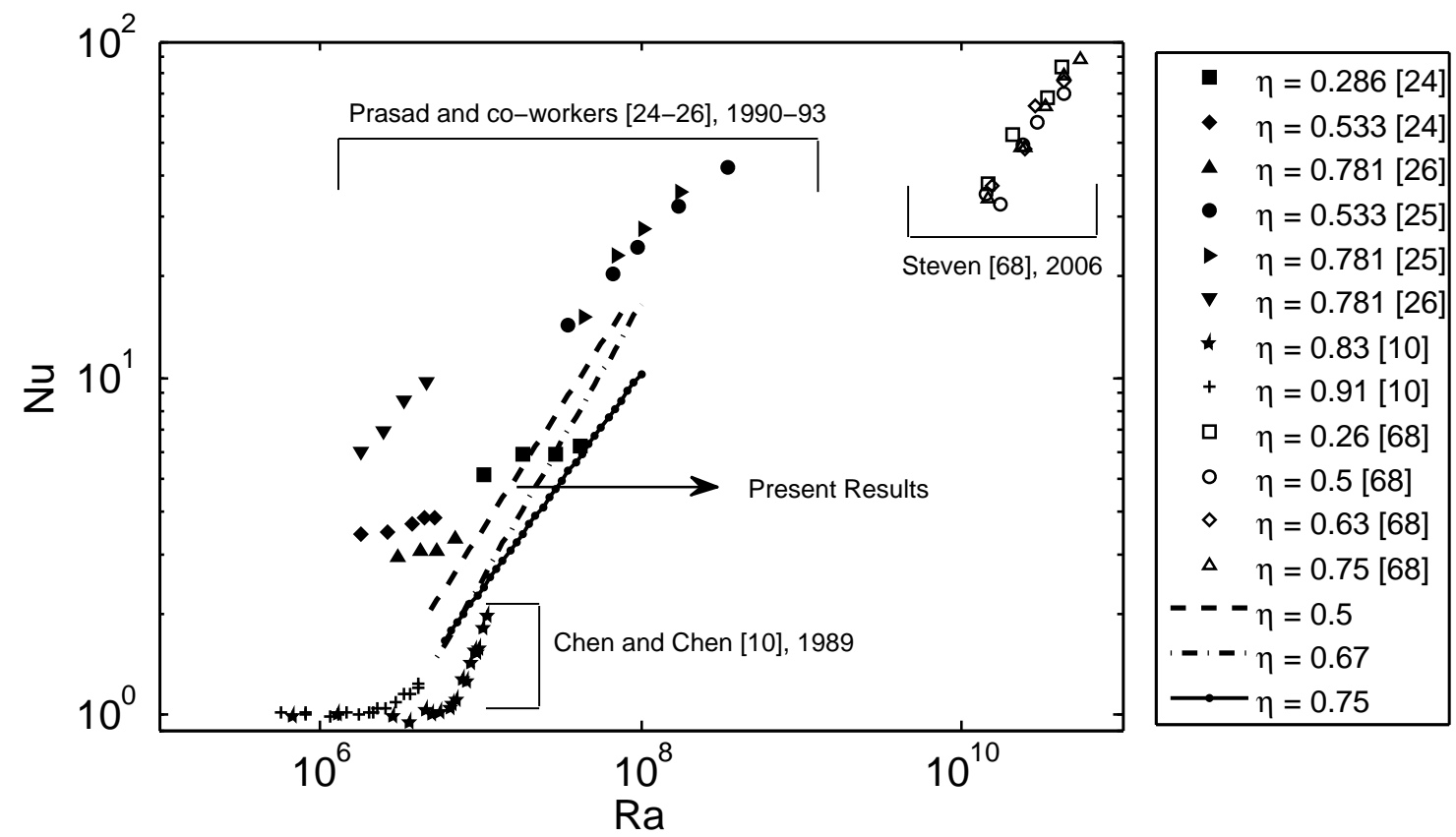

Figure 7.6: Comparison of present experimental results with all existing experiment studies.

a compilation of all experimental data published over the years. It can be readily seen that the data are scattered over a wide range of Rayleigh numbers and do not conform to any well defined pattern. This is in contrast to the similar Rayleigh-Bénard and HortonRogers-Lapwood problems for which the experimental data follow a coherent pattern which hints towards a fundamental underlying relation. Part of this can be attributed to the complexity of the dual-layer problem because of the large number of independent controlling parameters. However, part of the reason why the overall nature of the problem is not yet well understood is that previous studies have focused on studying very specific aspects of the problem. Taken in this context, the combined numerical-experimental approach that is adopted in this thesis represents an important step towards fundamentally understanding the problem over a large portion of the entire heat transfer regime. Also, this thesis has studied a new subsection of the dual-layer problem by investigating the effects of localized heat sources which are more relevant from an engineering perspective.

\subsection{Recommendations for future work}

The discussions in this chapter point to the fact that the problem of convection in fluid-superposed porous layers is a challenging one. Many aspects of this problem are still to be resolved and this problem remains an area of active investigation. Based on the results of this thesis, the following recommendations are proposed: 
- The numerical results presented in the present study can be improved by modifying the code to account for the discontinuity of properties at the interface. The approach of using Heaviside step functions to model properties such as the porosity, permeability, and thermal diffusivity proposed by Hirata et al [19]. should be incorporated in to the model.

- Simulations presented in the study can be extended by using a constant heat flux boundary condition at the heated surface instead of an isothermally heated central section. This approach will allow direct comparison with experimental data.

- Experiments can be extended by studying the effects of independent parameters such as the conductivity ratio, $\lambda$, Prandtl number, $\operatorname{Pr}$ and the aspect ratio.

- Experiments with highly porous and permeable media can be performed as these have important practical applications. These experiments will also allow one to study the effect of fluid inertia and possible transitions to turbulent flow regimes.

- An important extension of the experimental study is to perform flow visualization experiments with extremely narrow porous layers. These experiments can reveal to what extent, if at all, flow penetration from to the fluid to the porous layer takes place.

- Finally, careful experiments are needed for determining the critical Rayleigh number for the onset of convection. These experiments can confirm the findings of Chen and Chen [10]. They can also be used to validate the different mathematical models as several studies on the stability problem have already been published. 


\section{References}

[1] Neild, D. A. and Bejan, A. (1999). Convection in porous media (2nd ed.). New York, NY: Springer-Verlag.

[2] Horton, C. W. and Rogers Jr., F. T (1945). Convection currents in a porous medium. Journal of Applied Physics, 16(6), 367-370.

[3] Lapwood, E. R. (1948). Convection of a fluid in a porous medium. Mathematical Proceedings of the Cambridge Philosophical Society, 44 (4), 508-521.

[4] Combarnous, M. A. and Bories, S. (1975). Hydrothermal convection in saturated porous media. Advances in Hydroscience, 10, 231-307.

[5] Cheng, P. (1978). Heat transfer in geothermal systems. Advances in Heat Transfer, 14, 1-105.

[6] Kaviany, M. (1991). Principles of heat transfer in porous media. New York, NY: Springer-Verlag.

[7] Sun, W. J. (1973). Convective Instability in Superposed Porous and Free Layers. PhD thesis, University of Minnesota.

[8] Nield, D. A. (1977). Onset of convection in a fluid layer overlying a layer of a porous medium. Journal of Fluid Mechanics, $81(3)$, 513-522.

[9] Chen, F. and Chen, C. F. (1988). Onset of finger convection in a horizontal porous layer underlying fluid layer. Journal of Heat Transfer, 403, 403-409.

[10] Chen, F. and Chen, C. F. (1989). Experimental investigation of convective stability in a superposed fluid and porous layer when heated from below. Journal of Fluid Mechanics, 20\%, 311-321.

[11] Chen, F. (1991). Throughflow effects on convective instability in superposed fluid and porous layers. Journal of Fluid Mechanics, 231, 113-133. 
[12] Chen, F., Chen, C. F. and Pearlstein, A. J. (1991). Convective instability in superposed fluid and anisotropic porous layers. Physics of Fluids A: Fluid Dynamics, 3(4), 556-565.

[13] Chen, F. and Hsu, L. H. (1991). Onset of thermal convection in an anisotropic and inhomogeneous porous layer underlying a fluid layer. Journal of Applied Physics, 69(9), 6289-6301.

[14] Chen, F. and Lu, J. W. (1992). Variable viscosity effects on convective instability in superposed fluid and porous layers. Physics of Fluids A: Fluid Dynamics, 4(9), 1936-1944.

[15] Zhao, P. and Chen, C. F. (2001). Stability analysis of double-diffusive convection in superposed fluid and porous layers using a one-equation model. International Journal of Heat and Mass Transfer, 44, 4625-4633.

[16] Hirata, S. C., Goyeau, B., Gobin, D. and Cotta, R. M. (2006). Stability of natural convection in superposed fluid and porous layers using integral transforms. Numerical Heat Transfer, 50(5), 409-424.

[17] Hirata, S. C., Goyeau, B., Gobin, D., Carr, M. and Cotta, R. M. (2007). Linear stability of natural convection in superposed fluid and porous layers: Influence of the interfacial modeling. International Journal of Heat and Mass Transfer, 50(7), 1356-1367.

[18] Hirata, S. C., Goyeau, B. and Gobin, D. (2007). Stability of natural convection in superposed fluid and porous layers: Influence of the interfacial jump boundary condition. Physics of Fluids, 19(5), 058102(1-4).

[19] Hirata, S. C., Goyeau, B., Gobin, D., Chandesris, M. and Jamet, D. (2009). Stability of natural convection in superposed fluid and porous layers: Equivalence of the oneand two-domain approaches. International Journal of Heat and Mass Transfer, 52(7), 533-536.

[20] Poulikakos, D., Bejan, A., Selimos, B. and Blake, K. R. (1986). High Rayleigh number convection in a fluid overlaying a porous bed. International Journal of Heat and Fluid Flow, 7(2), 109-116.

[21] Poulikakos, D. (1986). Buoyancy driven convection in a horizontal fluid layer extending over a porous substrate. Physics of Fluids, 29(12), 3949-3957. 
[22] Chen, F. and Chen, C. F. (1992). Natural convection in superposed fluid and porous layers. Journal of Fluid Mechanics, 234, 97-119.

[23] Kim, S. J. and Choi, C. Y. (1996). Convective heat transfer in porous and overlying fluid layers heated from below. International Journal of Heat and Mass Transfer, $39(2), 319-329$.

[24] Prasad, V. and Tian, Q. (1990). An experimental study of thermal convection in fluid-superposed porous layers heated from below. Heat Transfer 1990, 5, $207-212$.

[25] Prasad, V., Brown, K. and Tian, Q. (1991). Flow visualization and heat transfer experiments in fluid-superposed packed beds heated from below. Experimental Thermal and Fluid Science, 4(1), 12- 24.

[26] Prasad, V. (1993). Flow instabilities and heat transfer in fluid overlying horizontal porous layers. Experimental Thermal and Fluid Science, 6(2), 135 - 146.

[27] Aichlmayr, H. T. (1999). The effective thermal conductivity of saturated porous media. Master's thesis, University of Minnesota.

[28] Schneider, K. J. (1963). Investigation on the influence of free thermal convection on heat transfer through granular material. Proceedings of the 11th International Congress of Refrigeration, 247-253.

[29] Elder, J. W. (1967). Steady free convection in a porous medium heated from below. Journal of Fluid Mechanics, 27(1), 29-48.

[30] Katto, Y. and Masuoka, Y. (1967). Criterion for the onset of convective flow in a fluid in a porous medium. International Journal of Heat and Mass Transfer, 10(3), 297-309.

[31] Masuoka, T. (1972). Heat transfer by free convection in a porous layer heated from below. Heat Transfer - Japan Research, 1, 39-45.

[32] Combarnous, M. A. and LeFur, B. (1969). Transfert de chaleur par convection naturelle dans une couche poreuse horizontale. Comptes Rendus de l'Acadmie des Sciences Paris Series B, 269, 1009-1012.

[33] Combarnous, M. A. (1970). Convection naturelle et convection mixte dans une couche poreuse horizontale. Revue Generale de Thermique, 9, 1355-1375.

[34] Bories, S. (1970). Sur la mechanismes fondamentaux de la convection naturelle en milieu poreux. Revue Generale de Thermique, 9, 1377-1401. 
[35] Kaneko, T., Mohtadi, M. F. and Aziz, K. (1974). An experimental study of natural convection in inclined porous media. International Journal of Heat and Mass Transfer, $17(4), 485-496$.

[36] Yen, Y. C. (1974). Effects of density inversion on free convective heat transfer in porous layer heated from below. International Journal of Heat and Mass Transfer, $17(11), 1349-1356$.

[37] Buretta, R. J. (1972). Thermal convection in a fluid filled porous layer with uniform internal heat sources. PhD thesis, University of Minnesota.

[38] Buretta, R. J. and Berman, A. S. (1976). Convective heat transfer in a liquid saturated porous layer. Journal of Applied Mechanics, 98, 249-253.

[39] Busse, F. H. and Joseph, D. D. (1972). Bounds for heat transport in a porous layer. Journal of Fluid Mechanics, 54(3), 521-543.

[40] Gupta, V. P. and Joseph, D. D. (1973). Bounds for heat transport in a porous layer. Journal of Fluid Mechanics, 57(3), 491-514.

[41] Seki, N., Fukusako, S. and Ariake, Y. (1980). Experimental study of free convective heat transfer in a liquid-saturated porous bed at high Rayleigh number. Heat and Mass Transfer, 13(1-2), 61-71.

[42] Combarnous, M. A. and Bories, S. (1974). Modeliazation de la convection naturelle au sein d'une couce poreuse horizontale a l'aide d'um coefficient de transfet solide-fluide. International Journal of Heat and Mass Transfer, 17, 505-515.

[43] Prasad, V. (1983). Natural convection in porous media - An experimental and numerical study for vertical annular and rectangular enclosures. PhD thesis, University of Delaware.

[44] Prasad, V., Kulacki, F. A. and Keyhani, M. (1985). Natural convection in porous media. Journal of Fluid Mechanics, 150, 89-119.

[45] Somerton, C. W. (1985). The Prandtl number effect in porous layer convection. Applied Scientific Research, 40(4), 333-344.

[46] Jonsson, T. and Catton, I. (1987). Prandtl number dependence of natural convection in porous media. Journal of Heat Transfer, 109(1), 371-377.

[47] Georgiadis, J. G. and Catton, I. (1986). Prandtl number effect on Bénard convection in porous media. Journal of Heat Transfer, 108(2), 284-290. 
[48] Catton, I. (1985). Natural convection heat transfer in porous media. In Kakac, S., Aung, W. and Viskanta, R. (Eds.), Natural Convection: Fundamentals and Applications (pages 514-547). Washington DC: Hemisphere.

[49] Close, D. J. (1986). A general correlation for natural convection in liquid-saturated beds of spheres. Journal of Heat Transfer, 108(4), 983-985.

[50] Yagi, S. and. Kunii, D. (1957). Studies on effective thermal conductivities in packed beds. AIChE Journal, 3(3), 373-381.

[51] Wang, M. and Bejan, A. (1987). Heat transfer correlation for Bénard convection in a fluid saturated porous layer. International Communications in Heat and Mass Transfer, 14, 617-626.

[52] Kladias, N. and Prasad, V. (1989). Natural convection in horizontal porous layers: effects of Darcy and Prandtl numbers. Journal of Heat Transfer, 111(4), 926-935.

[53] Kladias, N. and Prasad, V. (1991). Experimental verification of Darcy-BrinkmanForchheimer flow model for natural convection in porous media. Journal of Thermophysics and Heat Transfer, 5(4), 560-576.

[54] Elder, J. W. (1967). Transient conduction in porous media. Journal of Fluid Mechanics, $27(3), 607-629$.

[55] Horne, R. N. and O' Sullivan, M. J. (1974). Oscillatory convection in a porous medium heated from below. Journal of Fluid Mechanics, 66(2), 339-352.

[56] Horne, R. N. and O' Sullivan, M. J. (1978). Origin of oscillatory convection in a porous medium heated from below. Physics of Fluids, 21(8), 1260-1264.

[57] Prasad, V. and Kulacki, F. A. (1987). Natural convection in horizontal porous layers with localized heating from below. Journal of Heat Transfer, 109(3), 795-798.

[58] Prasad, V. and Kulacki, F. A. (1986). Effects of the size of heat source on natural convection in horizontal porous layers heated from below. Heat Transfer 1986, 5, $2677-2682$.

[59] El-Khatib, A. and Prasad, V. (1987). Effects of stratification on heat transfer in horizontal porous layers locally heated from below. Journal of Heat Transfer, 109(2), 795-798.

[60] Rajen, G. and Kulacki, F. A. (1987). Natural convection in a porous layer locally heated from below - a regional laboratory model for a nuclear waste repository. ASME HTD, 67, 19-26. 
[61] Lai, F. C. and. Kulacki, F. A. (1991). Experimental study of free and mixed convection in horizontal porous layers locally heated from below. International Journal of Heat and Mass Transfer, $34(2), 525-541$.

[62] Lai, F. C. and. Kulacki, F. A. (1987). Natural convection in layered porous media partially heated from below. ASME HTD, 76, 27-36.

[63] Jendoubi, S. and Kulacki, F. A. (1999). Convection in layered porous media: A comparison of boundary heating methods. Proceedings of the 5th ASME/JSME Joint Thermal Engineering Conference, 1-14.

[64] Beavers, G. S and Joseph, D. D. (1977). Boundary conditions at a naturally permeable wall. Journal of Fluid Mechanics, 30(1), 197-207.

[65] Rhee, S. J., Dhir, V. K. and Catton, I. (1978). Natural convection heat transfer in beds of inductively heated particles. Journal of Heat Transfer, 100, 78-85.

[66] Somerton, C. W. and Catton, I. (1982). Thermal instability of superposed porous and fluid layers. Journal of Heat Transfer, 104(1), 160-165.

[67] Carr, M. and Straughan, B. (2003). Penetrative convection in a fluid overlying a porous layer. Advances in Water Resources, 26(3), $263-276$.

[68] Steven, S. (2006). Experimental study of free convection in fluid porous layers heated from below. Master's thesis, University of Minnesota.

[69] Ochoa-Tapia, J. A. and Whitaker, S. (1995). Momentum transfer at the boundary between a porous medium and a homogeneous fluid - I. Theoretical development. International Journal of Heat Mass Transfer, 38(14), 2635-2646.

[70] Ochoa-Tapia, J. A and Whitaker, S. (1995). Momentum transfer at the boundary between a porous medium and a homogeneous fluid - II. Comparison with experiment. International Journal of Heat Mass Transfer, 38(14), 2647-2655.

[71] Kataoka, I. (1986). Local instant formulation of two-phase flow. International Journal of Multiphase Flow, 12(5), 745-758.

[72] Singh, A. K. and Thorpe, G. R. (1995). Natural convection in a confined fluid overlying a porous layer - A comparison study of different models. Indian Journal of Pure and Applied Mathematics, 26(1), 81-95.

[73] Spalding, D. B. (1972). A novel finite-difference formulation for differential expressions involving both first and second order derivatives. International Journal for Numerical Methods in Engineering. 4, 551-555. 
[74] Patankar, S. (1980). Numerical heat transfer and fluid flow. Washington D.C.: Hemisphere.

[75] Leonard, B. P. (1979). A stable and accurate convective modeling procedure based on quadratic upstream interpolation. Computational Methods Applications in Mechanical Engineering, 19, 59-98.

[76] Leschziner, M. A. (1980). Practical evaluation of three finite-difference schemes for the computation of steady-state recirculating flows. Computer Methods in Applied Mechanics and Engineering, 23, 293-312.

[77] Leschziner, M. A. and Rodi, W. (1981). Calculation of annular and twin parallel jets using various discretization schemes and turbulence model variants. ASME Journal of Fluids Engineering, 103, 352-360.

[78] Han, T., Humphrey, J. A. C. and Launder, B. E. (1981). A comparison of hybrid and quadratic upstream differencing in high Reynolds number elliptic flows. Computer Methods in Applied Mechanics and Engineering, 29, 81-95.

[79] LeQuere, P., Humphrey, J. A. C. and Sherman, F. (1981). Numerical calculation of thermally driven two-dimensional unsteady laminar flow in a cavity of rectangular cross section. Numerical Heat Transfer, 4, 249-283.

[80] Chapman, R. S and Kuo, C. Y. (1983). Application of a high accuracy finite difference technique to steady, free surface flow problems. International Journal of Numerical Methods in Fluids, 3, 583-590.

[81] Hayase, T., Humphrey, J. A. C. and Grief, R. (1992). A consistently formulated QUICK scheme for fast and stable convergence using finite-volume iterative calculation procedures. Journal of Computational Physics, 98, 108-118.

[82] Orszag, S. A. and Israeli, M. (1974). Numerical simulations of viscous imcompressible flows. Annual Review of Fluid Mechanics, 6, 281-319.

[83] Weinan, E. and Liu, J-G. (1996). Vorticity boundary conditions and related issues for finite-difference schemes. Journal of Computational Physics, 24, 368-382.

[84] Davis, T. A. (1974). Direct methods for sparse linear systems. Philadelphia, PA: SIAM.

[85] Clever, R. M. and Busse, F. H. (1974). Transition to time-dependent convection. Journal of Fluid Mechanics, 6, 625-635. 
[86] Ouertatani, N., Cheikh, N. B., Beya, B. B. and Lili, T. (2008). Numerical simulation of two-dimensional Rayleigh-Bénard convection in an enclosure. Comptes Rendus Mecanique, 336(5), 464-470.

[87] Hollands, K. G. T., Raithby, G. D. and Konicek, L. (1975). Correlation equations for free convection heat transfer in horizontal layers of air and water. International Journal of Heat Mass Transfer, 18, 879-884.

[88] Caltagirone, J. P. (1975). Thermoconvective instabilities in a horizontal porous layer. Journal of Fluid Mechanics, 72(20), 269-287.

[89] Kunii, D. and Smith, J. M. (1960). Heat transfer characteristics of porous rocks. AIChE Journal, 6(1), 71-78.

[90] Amestoy, P., Davis, T. A. and Duff, I. S. (2004). Algorithm 837: AMD, An approximate minimum degree ordering algorithm. ACM Transactions on Mathematical Software, 30(3), 381-388.

[91] Chen, Y., Davis, T. A., Hager, W.W. and Rajamanickam, S. (2009). Algorithm 887: CHOLMOD, Supernodal Sparse Cholesky Factorization and Update/Downdate. ACM Transactions on Mathematical Software, 35(3), 1-14.

[92] Blackford, L. S., Demmel, J., Dongarra, J., Duff, I., Hammarling, S., Henry, G., Heroux, M., Kaufman, L., Lumsdaine, A., Petitet, A., Pozo, R., Remington, K., Whaley, R. C. (2002). An Updated Set of Basic Linear Algebra Subprograms (BLAS). ACM Transactions on Mathematical Software, 28(2), 135-151.

[93] Lemmon, E., McLinden, M., and Friend, D. (2003). Neutral Thermochemical Data. In P.J. Linstrom and W.G. Mallard (Eds.), NIST Chemistry WebBook, NIST Standard Reference Database Number 69. Gaithersburg, MD: National Institute of Standards and Technology. Retrieved from http://webbook.nist.gov.

[94] Lide, D. R. (2007). CRC Handbook of Chemistry and Physics (88th ed.). Boca Raton, FL: CRC Press.

[95] Topping, J. (1963). Errors of observation and their treatment (3rd ed.). London: Chapman and Hall Ltd. 


\section{Appendix A}

\section{Direct solver for the linear equations}

The basic form of the linear equations obtained be the discretization process is

$$
M \Theta=b,
$$

where $\Theta$ is either the vorticity, stream function or temperature. In this study, the direct solution of equation (A.1) is performed using the up-looking Cholesky factorization technique. The primary advantage of this technique is that it is very efficient for vary large sparse matrices. This method involves three primary steps: i) the selection of permutation matrices, ii) matrix factorization, and iii) final solution using back-substitution. The implementation of the individual sub-routines is performed using algorithms distributed via the open-source packages AMD [90], CHOLMOD [91] and BLAS [92]. The individual packages are accessed using the MATLAB interface. The different packages used in the individual steps are given in Table A1.

The general solution procedure is fairly straightforward and involves an up-looking Cholesky factorization of the matrix $M$ which gives

$$
P^{\prime} \cdot M \cdot P=R^{\prime} \cdot R
$$

where $P$ is a suitably selected permutation matrix, $R$ is an upper triangular matrix and $P^{\prime}$ and $R^{\prime}$ are the transposes of matrices $P$ and $R$, respectively. This process is actually a combination of the steps i) and ii) described above. Here, the permutation matrix, $P$, is first selected by calling the AMD package

$$
P=\operatorname{amd}(M) .
$$

The above algorithm returns the approximate minimum degree permutation vector for the sparse matrix $U=M+M^{\prime}$, where $M^{\prime}$ is the transpose of matrix $M$. This process speeds 
Table A.1: Packages used in the different steps of the direct solution.

\begin{tabular}{ccc}
\hline Solution Step & Package & Sub-routine \\
\hline \hline Permutation matrix selection & AMD & amd \\
Up-looking Cholesky factorization & CHOLMOD & chol \\
Back-substitution & BLAS & DTRSV, DTRSM \\
\hline
\end{tabular}

up the solution process as the Cholesky factorization of $P \cdot M \cdot P^{\prime}$ is considerably sparser than that of $M$ itself. In the next step, the factorization is performed as

$$
\operatorname{chol}\left(P^{\prime} \cdot M \cdot P\right)=R^{\prime} \cdot R
$$

Once the factorization is complete, the solution is obtained by the back-substitution processes,

$$
\Theta=P \cdot\left(R \backslash\left(R^{\prime} \backslash\left(P^{\prime} \cdot b\right)\right)\right),
$$

where the backslash represents a back-substitution. The back-substitution is performed using the sub-routines DTRSV and DTRSM of the BLAS package. 


\section{Appendix B}

\section{Data Tables}

Data from the numerical simulations and experiments are listed here. For the simulations, the Nusselt number is given at different values of the Rayleigh number and the independent controlling parameters. For experimental data, all measured quantities are listed. All data are available on a CD-ROM from the author.

Table B.1: Numerical Nusselt-Rayleigh number data for different values of $\delta$.

\begin{tabular}{cccc}
\hline$R a$ & $\delta=1$ & $\delta=0.5$ & $\delta=0.25$ \\
\hline \hline $1 \times 10^{3}$ & 1 & 1.6688 & 2.5492 \\
$1.5 \times 10^{3}$ & 1 & 1.6693 & 2.5501 \\
$2.5 \times 10^{3}$ & 1 & 1.6713 & 2.5534 \\
$5 \times 10^{3}$ & 1 & 1.6981 & 2.5825 \\
$7.5 \times 10^{3}$ & 1.0065 & 1.8308 & 2.6585 \\
$1 \times 10^{4}$ & 1.3242 & 2.1193 & 2.8646 \\
$1.5 \times 10^{4}$ & 1.675 & 2.5786 & 3.3137 \\
$2.5 \times 10^{4}$ & 2.0265 & 3.0681 & 3.8296 \\
$5 \times 104$ & 2.4707 & 3.6985 & 4.4881 \\
$1 \times 10^{5}$ & 2.9138 & 4.3302 & 5.1525 \\
$1.5 \times 10^{5}$ & 3.1787 & 4.7052 & 5.553 \\
$2.5 \times 10^{5}$ & 3.5233 & 5.187 & 6.0724 \\
$5 \times 105$ & 4.0167 & 5.864 & 6.8064 \\
$1 \times 10^{6}$ & 4.4995 & 6.5294 & 7.5335 \\
\hline
\end{tabular}


Table B.2: Numerical Nusselt-Rayleigh number data for different values of $\eta$.

\begin{tabular}{cccc}
\hline$R a$ & $\eta=0.25$ & $\eta=0.5$ & $\eta=0.75$ \\
\hline \hline $1 \times 10^{3}$ & 1.6795 & 1.6688 & 1.6684 \\
$1.5 \times 10^{3}$ & 1.7305 & 1.6693 & 1.6684 \\
$2.5 \times 10^{3}$ & 1.9375 & 1.6713 & 1.6684 \\
$3.5 \times 10^{3}$ & 2.2003 & - & - \\
$5 \times 10^{3}$ & 2.6553 & 1.6981 & 1.6684 \\
$7.5 \times 10^{3}$ & 3.1194 & 1.8308 & - \\
$1 \times 10^{4}$ & 3.9054 & 2.1193 & 1.6684 \\
$1.5 \times 10^{4}$ & 4.3688 & 2.5786 & 1.6685 \\
$2.5 \times 10^{4}$ & 4.9631 & 3.0681 & 1.6688 \\
$5 \times 104$ & 5.8076 & 3.6985 & 1.6711 \\
$1 \times 10^{5}$ & 6.6954 & 4.3302 & 1.6982 \\
$1.5 \times 10^{5}$ & 7.2274 & 4.7052 & 1.9898 \\
$2.5 \times 10^{5}$ & 7.8985 & 5.187 & 2.4059 \\
$5 \times 105$ & 8.7552 & 5.864 & 2.9579 \\
$1 \times 10^{6}$ & 9.6391 & 6.5294 & 3.52 \\
\hline
\end{tabular}

Table B.3: Numerical Nusselt-Rayleigh number data for different values of $A$.

\begin{tabular}{cccc}
\hline$R a$ & $A=2$ & $A=4$ & $A=6$ \\
\hline \hline $1 \times 10^{3}$ & 1.6688 & 1.4315 & 1.3023 \\
$1.5 \times 10^{3}$ & 1.6693 & 1.433 & 1.3034 \\
$2.5 \times 10^{3}$ & 1.6713 & 1.4381 & 1.3071 \\
$5 \times 10^{3}$ & 1.6981 & 1.4763 & 1.3332 \\
$7.5 \times 10^{3}$ & 1.8308 & 1.5626 & 1.45 \\
$1 \times 10^{4}$ & 2.1193 & 1.8441 & 1.7249 \\
$1.5 \times 10^{4}$ & 2.5786 & 2.3023 & 2.1389 \\
$2.5 \times 10^{4}$ & 3.0681 & 2.8342 & 2.5675 \\
$5 \times 104$ & 3.6985 & 3.5506 & 3.1259 \\
$1 \times 10^{5}$ & 4.3302 & 4.1792 & 3.6679 \\
$1.5 \times 10^{5}$ & 4.7052 & 4.5301 & 3.9689 \\
$2.5 \times 10^{5}$ & 5.187 & 4.9674 & 4.303 \\
$5 \times 105$ & 5.864 & 5.5437 & 5.1303 \\
$1 \times 10^{6}$ & 6.5294 & 6.1809 & 5.6898 \\
\hline
\end{tabular}


Table B.4: Numerical Nusselt-Rayleigh number data for different values of $D a$.

\begin{tabular}{cccc}
\hline$R a$ & $D a=10^{-2}$ & $D a=10^{-4}$ & $D a=10^{-6}$ \\
\hline \hline $1 \times 10^{2}$ & 1.6685 & - & - \\
$2.5 \times 10^{2}$ & 1.6688 & - & - \\
$5 \times 10^{2}$ & 1.6697 & - & - \\
$7.5 \times 10^{2}$ & 1.6713 & - & - \\
$1 \times 10^{3}$ & 1.674 & 1.6689 & 1.6688 \\
$1.5 \times 10^{3}$ & 1.6855 & 1.6696 & 1.6693 \\
$2.5 \times 10^{3}$ & 1.7988 & 1.6725 & 1.6713 \\
$3.5 \times 10^{3}$ & 2.123 & - & - \\
$5 \times 10^{3}$ & 2.5695 & 1.7204 & 1.6981 \\
$7.5 \times 10^{3}$ & 3.0433 & 2.0056 & 1.8308 \\
$1 \times 10^{4}$ & 3.3523 & 2.2959 & 2.1193 \\
$1.5 \times 10^{4}$ & 3.7649 & 2.7178 & 2.5786 \\
$2.5 \times 10^{4}$ & 4.2738 & 3.2072 & 3.0681 \\
$5 \times 104$ & 4.9871 & 3.855 & 3.6985 \\
$1 \times 10^{5}$ & 5.7606 & 4.5277 & 4.3302 \\
$1.5 \times 10^{5}$ & 6.2514 & 4.9444 & 4.7052 \\
$2.5 \times 10^{5}$ & 6.918 & 5.5089 & 5.187 \\
$5 \times 105$ & 7.9205 & 6.3523 & 5.864 \\
$1 \times 10^{6}$ & 9.05 & 7.3862 & 6.5294 \\
\hline
\end{tabular}


Table B.5: Numerical Nusselt-Rayleigh number data for different values of $\lambda$.

\begin{tabular}{cccc}
\hline$R a$ & $\lambda=1$ & $\lambda=50$ & $\lambda=100$ \\
\hline \hline $1 \times 10^{3}$ & 1.6688 & 3.3011 & 3.4284 \\
$1.5 \times 10^{3}$ & 1.6693 & 3.3013 & 3.4285 \\
$2.5 \times 10^{3}$ & 1.6713 & 3.3022 & 3.4292 \\
$5 \times 103$ & 1.6981 & 3.3151 & 3.4389 \\
$7.5 \times 10^{3}$ & 1.8308 & 3.4913 & 3.5897 \\
$1 \times 10^{4}$ & 2.1193 & 4.2054 & 4.3267 \\
$1.5 \times 10^{4}$ & 2.5786 & 5.3623 & 5.529 \\
$2.5 \times 10^{4}$ & 3.0681 & 6.6362 & 6.9038 \\
$5 \times 104$ & 3.6985 & 8.2186 & 8.6373 \\
$1 \times 10^{5}$ & 4.3302 & 9.7408 & 10.3184 \\
$1.5 \times 10^{5}$ & 4.7052 & 10.6266 & 11.3004 \\
$2.5 \times 10^{5}$ & 5.187 & 11.7567 & 12.5532 \\
$5 \times 105$ & 5.864 & 13.3573 & 14.3257 \\
$1 \times 10^{6}$ & 6.5294 & 14.9801 & 16.1335 \\
\hline
\end{tabular}

Table B.6: Numerical Nusselt-Rayleigh number data for different values of $\operatorname{Pr}$.

\begin{tabular}{ccc}
\hline$R a$ & $P r=0.7$ & $\operatorname{Pr}=7$ \\
\hline \hline $1 \times 10^{3}$ & 1.6688 & 1.6688 \\
$1.5 \times 10^{3}$ & 1.6693 & 1.6693 \\
$2.5 \times 10^{3}$ & 1.6713 & 1.6713 \\
$5 \times 103$ & 1.6981 & 1.6981 \\
$7.5 \times 10^{3}$ & 1.8959 & 1.8308 \\
$1 \times 10^{4}$ & 2.1496 & 2.1193 \\
$1.5 \times 10^{4}$ & 2.5672 & 2.5786 \\
$2.5 \times 10^{4}$ & 3.0387 & 3.0681 \\
$5 \times 104$ & 3.6333 & 3.6985 \\
$1 \times 10^{5}$ & 4.2239 & 4.3302 \\
$1.5 \times 10^{5}$ & 4.5726 & 4.7052 \\
$2.5 \times 10^{5}$ & 5.0129 & 5.187 \\
$5 \times 105$ & 5.6044 & 5.864 \\
$1 \times 10^{6}$ & 6.1349 & 6.5294 \\
\hline
\end{tabular}


Table B.7: Measured experimental parameters for $\delta=0.44$ and $\eta=0.5$.

\begin{tabular}{cccccc}
\hline Run No. & $T_{H, \text { avg }}$ & $T_{C, \text { avg }}$ & $T_{G, \text { avg }}$ & $V$ & $V_{G}$ \\
\hline \hline 1 & 27.612 & 22.54 & 27.71 & 17.98 & 4.55 \\
2 & 33.198 & 22.144 & 33.238 & 32.86 & 7.49 \\
3 & 37.24 & 22.174 & 37.69 & 48.04 & 7.3 \\
4 & 42.298 & 22.454 & 42.88 & 69.71 & 4.54 \\
5 & 49.608 & 22.99 & 50.323 & 94.69 & 1.83 \\
6 & 53.316 & 22.902 & 53.503 & 105.02 & 1.84 \\
7 & 59.914 & 23.876 & 60.495 & 126.89 & 0.005 \\
\hline
\end{tabular}

Table B.8: Measured experimental parameters for $\delta=0.44$ and $\eta=0.67$.

\begin{tabular}{cccccc}
\hline Run No. & $T_{H, \text { avg }}$ & $T_{C, \text { avg }}$ & $T_{G, \text { avg }}$ & $V$ & $V_{G}$ \\
\hline \hline 1 & 27.87 & 22.274 & 27.51 & 18.59 & 4.84 \\
2 & 34.798 & 22.572 & 34.15 & 35.54 & 8.36 \\
3 & 38.58 & 22.792 & 37.988 & 49.37 & 7.37 \\
4 & 43.722 & 22.5 & 44.405 & 73.56 & 1.827 \\
5 & 48.946 & 23.356 & 50.485 & 91.76 & 4.54 \\
6 & 60.47 & 23.954 & 60.675 & 129.93 & 0.005 \\
7 & 54.764 & 23.438 & 55.725 & 111.85 & 0.005 \\
\hline
\end{tabular}

Table B.9: Measured experimental parameters for $\delta=0.44$ and $\eta=0.75$.

\begin{tabular}{cccccc}
\hline Run No. & $T_{H, \text { avg }}$ & $T_{C, \text { avg }}$ & $T_{G, \text { avg }}$ & $V$ & $V_{G}$ \\
\hline \hline 1 & 29.572 & 23.532 & 28.57 & 18.69 & 3.875 \\
2 & 33.988 & 22.836 & 31.065 & 32.37 & 9.311 \\
3 & 39.11 & 22.942 & 38.338 & 45.87 & 13.153 \\
4 & 43.84 & 23.236 & 42.84 & 59.99 & 13.405 \\
5 & 52.286 & 25.046 & 50.848 & 78.21 & 15.223 \\
6 & 61.792 & 24.658 & 59.998 & 99.3 & 19.15 \\
\hline
\end{tabular}


Table B.10: Measured experimental parameters for $\delta=0.11$ and $\eta=0.5$.

\begin{tabular}{cccccc}
\hline Run No. & $T_{H, \text { avg }}$ & $T_{C, \text { avg }}$ & $T_{G, \text { avg }}$ & $V$ & $V_{G}$ \\
\hline \hline 1 & 29.572 & 23.532 & 28.57 & 18.69 & 3.875 \\
2 & 33.988 & 22.836 & 31.065 & 32.37 & 9.311 \\
3 & 39.11 & 22.942 & 38.338 & 45.87 & 13.153 \\
4 & 43.84 & 23.236 & 42.84 & 59.99 & 13.405 \\
5 & 52.286 & 25.046 & 50.848 & 78.21 & 15.223 \\
6 & 61.792 & 24.658 & 59.998 & 99.3 & 19.15 \\
\hline
\end{tabular}




\section{Appendix $\mathrm{C}$}

\section{Thermophysical Properties}

Thermophysical properties of all materials used in the experiments are listed here. All solid properties listed are evaluated at $25{ }^{\circ} \mathrm{C}$ and their sources are listed in Table B1. Fluid properties are evaluated at the mean temperature $T_{M}=\left(T_{H}+T_{C}\right) / 2$. All fluid properties are obtained from the NIST Standard Reference Database [93].

Table C.1: Thermophysical properties of solid materials.

\begin{tabular}{lcccc}
\hline Material & $\rho\left(\mathrm{kg} / \mathrm{m}^{3}\right)$ & $c_{p}(\mathrm{~J} / \mathrm{kg} \mathrm{K})$ & $k(\mathrm{~W} / \mathrm{m} \mathrm{K})$ & Source \\
\hline \hline Glass beads & 2500 & 918.2 & 0.64 & {$[94]$} \\
Polycarbonate & 1200 & 1250 & 0.2 & {$[94]$} \\
Polystyrene insulation & 1050 & 1300 & 0.08 & {$[94]$} \\
\hline
\end{tabular}

Table C.2: Properties of water for experiment with $\delta=0.44$ and $\eta=0.5$.

\begin{tabular}{cccccc}
\hline Run No. & $T_{M}\left({ }^{\circ} \mathrm{C}\right)$ & $k(\mathrm{~W} / \mathrm{m} \mathrm{K})$ & $\nu\left(\mathrm{m}^{2} / \mathrm{s}\right)$ & $\alpha\left(\mathrm{m}^{2} / \mathrm{s}\right)$ & $\beta(1 / \mathrm{K})$ \\
\hline \hline 1 & 25.08 & 0.61 & $8.911 \times 10^{-7}$ & $1.457 \times 10^{-7}$ & $2.580 \times 10^{-4}$ \\
2 & 27.67 & 0.61 & $8.416 \times 10^{-7}$ & $1.469 \times 10^{-7}$ & $2.820 \times 10^{-4}$ \\
3 & 29.71 & 0.62 & $8.057 \times 10^{-7}$ & $1.478 \times 10^{-7}$ & $3.010 \times 10^{-4}$ \\
4 & 32.38 & 0.62 & $7.625 \times 10^{-7}$ & $1.489 \times 10^{-7}$ & $3.240 \times 10^{-4}$ \\
5 & 36.3 & 0.63 & $7.056 \times 10^{-7}$ & $1.506 \times 10^{-7}$ & $3.560 \times 10^{-4}$ \\
6 & 38.11 & 0.63 & $6.817 \times 10^{-7}$ & $1.513 \times 10^{-7}$ & $3.710 \times 10^{-4}$ \\
7 & 41.9 & 0.63 & $6.358 \times 10^{-7}$ & $1.528 \times 10^{-7}$ & $4.000 \times 10^{-4}$ \\
\hline
\end{tabular}


Table C.3: Properties of water for experiment with $\delta=0.44$ and $\eta=0.67$.

\begin{tabular}{cccccc}
\hline Run No. & $T_{M}\left({ }^{\circ} \mathrm{C}\right)$ & $k(\mathrm{~W} / \mathrm{m} \mathrm{K})$ & $\nu\left(\mathrm{m}^{2} / \mathrm{s}\right)$ & $\alpha\left(\mathrm{m}^{2} / \mathrm{s}\right)$ & $\beta(1 / \mathrm{K})$ \\
\hline \hline 1 & 25.07 & 0.61 & $8.913 \times 10^{-7}$ & $1.457 \times 10^{-7}$ & $2.580 \times 10^{-4}$ \\
2 & 28.69 & 0.61 & $8.233 \times 10^{-7}$ & $1.473 \times 10^{-7}$ & $2.920 \times 10^{-4}$ \\
3 & 30.69 & 0.62 & $7.894 \times 10^{-7}$ & $1.482 \times 10^{-7}$ & $3.090 \times 10^{-4}$ \\
4 & 33.11 & 0.62 & $7.513 \times 10^{-7}$ & $1.492 \times 10^{-7}$ & $3.300 \times 10^{-4}$ \\
5 & 36.15 & 0.63 & $7.076 \times 10^{-7}$ & $1.505 \times 10^{-7}$ & $3.550 \times 10^{-4}$ \\
6 & 42.21 & 0.63 & $6.322 \times 10^{-7}$ & $1.529 \times 10^{-7}$ & $4.020 \times 10^{-4}$ \\
7 & 39.1 & 0.63 & $6.691 \times 10^{-7}$ & $1.517 \times 10^{-7}$ & $3.790 \times 10^{-4}$ \\
\hline
\end{tabular}

Table C.4: Properties of water for experiment with $\delta=0.44$ and $\eta=0.75$.

\begin{tabular}{cccccc}
\hline Run No. & $T_{M}\left({ }^{\circ} \mathrm{C}\right)$ & $k(\mathrm{~W} / \mathrm{m} \mathrm{K})$ & $\nu\left(\mathrm{m}^{2} / \mathrm{s}\right)$ & $\alpha\left(\mathrm{m}^{2} / \mathrm{s}\right)$ & $\beta(1 / \mathrm{K})$ \\
\hline \hline 1 & 26.55 & 0.61 & $8.624 \times 10^{-7}$ & $1.464 \times 10^{-7}$ & $2.720 \times 10^{-4}$ \\
2 & 28.41 & 0.61 & $8.283 \times 10^{-7}$ & $1.472 \times 10^{-7}$ & $2.890 \times 10^{-4}$ \\
3 & 31.03 & 0.62 & $7.838 \times 10^{-7}$ & $1.484 \times 10^{-7}$ & $3.120 \times 10^{-4}$ \\
4 & 33.54 & 0.62 & $7.448 \times 10^{-7}$ & $1.494 \times 10^{-7}$ & $3.340 \times 10^{-4}$ \\
5 & 38.67 & 0.63 & $6.745 \times 10^{-7}$ & $1.515 \times 10^{-7}$ & $3.750 \times 10^{-4}$ \\
6 & 43.23 & 0.64 & $6.209 \times 10^{-7}$ & $1.533 \times 10^{-7}$ & $4.100 \times 10^{-4}$ \\
\hline
\end{tabular}

Table C.5: Properties of water for experiment with $\delta=0.11$ and $\eta=0.5$.

\begin{tabular}{cccccc}
\hline Run No. & $T_{M}\left({ }^{\circ} \mathrm{C}\right)$ & $k(\mathrm{~W} / \mathrm{m} \mathrm{K})$ & $\nu\left(\mathrm{m}^{2} / \mathrm{s}\right)$ & $\alpha\left(\mathrm{m}^{2} / \mathrm{s}\right)$ & $\beta(1 / \mathrm{K})$ \\
\hline \hline 1 & 25.84 & 0.61 & $8.761 \times 10^{-7}$ & $1.460 \times 10^{-7}$ & $2.650 \times 10^{-4}$ \\
2 & 28.06 & 0.61 & $8.345 \times 10^{-7}$ & $1.470 \times 10^{-7}$ & $2.860 \times 10^{-4}$ \\
3 & 30.16 & 0.62 & $7.982 \times 10^{-7}$ & $1.480 \times 10^{-7}$ & $3.050 \times 10^{-4}$ \\
4 & 31.98 & 0.62 & $7.688 \times 10^{-7}$ & $1.488 \times 10^{-7}$ & $3.210 \times 10^{-4}$ \\
5 & 34.61 & 0.62 & $7.292 \times 10^{-7}$ & $1.499 \times 10^{-7}$ & $3.430 \times 10^{-4}$ \\
6 & 37.01 & 0.63 & $6.962 \times 10^{-7}$ & $1.543 \times 10^{-7}$ & $3.620 \times 10^{-4}$ \\
7 & 39.55 & 0.63 & $6.636 \times 10^{-7}$ & $1.519 \times 10^{-7}$ & $3.820 \times 10^{-4}$ \\
8 & 42.35 & 0.63 & $6.307 \times 10^{-7}$ & $1.530 \times 10^{-7}$ & $4.030 \times 10^{-4}$ \\
\hline
\end{tabular}




\section{Appendix D}

\section{Uncertainty Analysis}

The uncertainty analysis presented here is based on Topping [95]. The two primary quantities that need to be evaluated are the experimental uncertainties in the Nusselt and Rayleigh numbers.

\section{D.1 Uncertainty in Nusselt number}

Recalling the definition of the $N u$ given in equation (6.1), the uncertainty in $N u$ using the method of least squares is,

$$
\begin{aligned}
& U_{N u}^{2}=\left(\frac{\partial N u}{\partial q_{\text {net }}} U_{q_{\text {net }}}\right)^{2}+\left(\frac{\partial N u}{\partial H} U_{H}\right)^{2}+\left(\frac{\partial N u}{\partial k_{\mathrm{f}}} U_{k_{\mathrm{f}}}\right)^{2} \\
&+\left(\frac{\partial N u}{\partial \Delta T} U_{\Delta T}\right)^{2}+\left(\frac{\partial N u}{\partial A_{\mathrm{tot}}} U_{A_{\mathrm{tot}}}\right)^{2} \\
&=\left(\frac{H}{k_{\mathrm{f}} A_{\mathrm{tot}} \Delta T} U_{q_{\mathrm{net}}}\right)^{2}+\left(\frac{q_{\mathrm{net}}}{k_{\mathrm{f}} A_{\mathrm{tot}} \Delta T} U_{H}\right)^{2}+\left(-\frac{q_{\mathrm{net}} H}{k_{f}^{2} A_{\mathrm{tot}} \Delta T} U_{k_{\mathrm{f}}}\right)^{2} \\
& \quad+\left(-\frac{q_{\text {net }} H}{k_{\mathrm{f}} A_{\mathrm{tot}} \Delta T^{2}} U_{\Delta T}\right)^{2}+\left(-\frac{q_{\mathrm{net}} H}{k_{\mathrm{f}} A_{\mathrm{tot}}^{2} \Delta T} U_{\Delta T}\right)^{2} \\
&=\left(\frac{N u}{q_{\mathrm{net}}} U_{q_{\mathrm{net}}}\right)^{2}+\left(\frac{N u}{H} U_{H}\right)^{2}+\left(-\frac{N u}{k_{\mathrm{f}}} U_{k_{\mathrm{f}}}\right)^{2} \\
& \quad+\left(-\frac{N u}{\Delta T} U_{\Delta T}\right)^{2}+\left(-\frac{N u}{\Delta T} U_{\Delta T}\right)^{2} .
\end{aligned}
$$

The relative uncertainty in $N u$ is therefore,

$$
\left(\frac{U_{N u}}{N u}\right)^{2}=\left(\frac{U_{q_{\mathrm{net}}}}{q_{\mathrm{net}}}\right)^{2}+\left(\frac{U_{H}}{H}\right)^{2}+\left(\frac{U_{k_{\mathrm{f}}}}{k_{\mathrm{f}}}\right)^{2}+\left(\frac{U_{\Delta T}}{\Delta T}\right)^{2}+\left(\frac{U_{A_{\mathrm{tot}}}}{A_{\mathrm{tot}}}\right)^{2} .
$$




\section{D.2 Uncertainty in Rayleigh number}

Using the definition of $R a$ given in equation (D.2), the least square uncertainty in

$R a$ is,

$$
\begin{aligned}
U_{R a}^{2} & =\left(\frac{\partial R a}{\partial \beta} U_{\beta}\right)^{2}+\left(\frac{\partial R a}{\partial H} U_{H}\right)^{2}+\left(\frac{\partial R a}{\partial \Delta T} U_{\Delta T}\right)^{2}+\left(\frac{\partial R a}{\partial \nu} U_{\nu}\right)^{2}+\left(\frac{\partial R a}{\partial \alpha} U_{\alpha}\right)^{2} \\
& =\left(\frac{R a}{\beta} U_{\beta}\right)^{2}+\left(\frac{3 R a}{H} U_{H}\right)^{2}+\left(\frac{R a}{\Delta T} U_{\Delta T}\right)^{2}+\left(\frac{R a}{\nu} U_{\nu}\right)^{2}+\left(\frac{R a}{\alpha} U_{\alpha}\right)^{2} .
\end{aligned}
$$

Therefore the relative uncertainty in $R a$ is,

$$
\left(\frac{U_{R a}}{R a}\right)^{2}=\left(\frac{U_{\beta}}{\beta}\right)^{2}+\left(\frac{3 U_{H}}{H}\right)^{2}+\left(\frac{U_{\Delta T}}{\Delta T}\right)^{2}+\left(\frac{U_{\nu}}{\nu}\right)^{2}+\left(\frac{U_{\alpha}}{\alpha}\right)^{2} .
$$

\section{D.3 Uncertainty in thermophysical constants}

The uncertainty of the thermophysical constants is based on standard deviation of the polynomial equations used to calculate the value of the thermal expansion coefficient, $\beta$, density, $\rho$, specific heat, $c_{p}$, thermal conductivity, $k$, and dynamic viscosity, $\mu$. These uncertainty values are obtained from the data tables used to obtain the thermophysical data for water [93]. The uncertainties in the aforementioned quantities are:

- $U_{\beta}=7.766 \times 10^{-6} 1 / \mathrm{K}$.

- $U_{\mu}=1.186 \times 10^{-5} \mathrm{~kg} / \mathrm{m} \mathrm{s}$.

- $U_{\rho}=0.2797 \mathrm{~kg} / \mathrm{m}^{3}$.

- $U_{k_{\mathrm{f}}}=0.0041 \mathrm{~W} / \mathrm{m} \mathrm{K}$.

- $U_{c_{p}}=0.9144 \mathrm{~J} / \mathrm{kg} \mathrm{K}$.

The relative uncertainties in the derived thermophysical properties, namely the thermal diffusivity, $\alpha\left(k_{\mathrm{f}} / c_{p}\right)$, and the kinematic viscosity, $\nu(\mu / \rho)$, are,

$$
\begin{gathered}
\left(\frac{U_{\alpha}}{\alpha}\right)^{2}=\left(\frac{U_{k_{\mathrm{f}}}}{k_{\mathrm{f}}}\right)^{2}+\left(\frac{U_{\rho}}{\rho}\right)+\left(\frac{U_{c_{p}}}{c_{p}}\right)^{2}, \\
\left(\frac{U_{\nu}}{\nu}\right)^{2}=\left(\frac{U_{\mu}}{\mu}\right)^{2}+\left(\frac{U_{\rho}}{\rho}\right)^{2} .
\end{gathered}
$$




\section{D.4 Uncertainty in length and area measurements}

In the present study, the uncertainty in all length and height measurements is assumed to be $1 \mathrm{~mm}$. The relative uncertainty in the measurement of the heater area is,

$$
\left(\frac{U_{A_{\mathrm{tot}}}}{A_{\mathrm{tot}}}\right)^{2}=\left(\frac{U_{L_{H}}}{L_{H}}\right)^{2}+\left(\frac{U_{W_{H}}}{W_{H}}\right)^{2}
$$

\section{D.5 Uncertainty in thermocouple measurements}

The uncertainty in a thermocouple measurement is equal to the bias error of the measurements. The thermocouples used in these experiments are of the special limits of error grade which have reported uncertainty of $\pm 1.2{ }^{\circ} \mathrm{C}$ for type $\mathrm{E}$ thermocouples and $\pm 1.7^{\circ} \mathrm{C}$ for type $\mathrm{J}$ thermocouples.

\section{D.6 Uncertainty in $\Delta T$}

The least square uncertainty in $\Delta T$ is given as,

$$
U_{\Delta T}^{2}=\left(U_{T_{H, \text { avg }}}\right)^{2}+\left(U_{T_{C, \text { avg }}}\right)^{2}
$$

where $U_{T_{H, \text { avg }}}$ and $U_{T_{C, \text { avg }}}$ are the uncertainties in the average temperatures at the heater and top surfaces respectively and are

$$
\begin{gathered}
\left(U_{T_{H, \text { avg }}}\right)^{2}=\frac{U_{T}^{2}}{n_{H}}, \\
\left(U_{T_{C, \text { avg }}}\right)^{2}=\frac{U_{T}^{2}}{n_{C}},
\end{gathered}
$$

Here $U_{T}$ is the uncertainty in a single thermocouple measurement and $n_{H}$ and $n_{C}$ are the number of thermocouples on the heater and top surface respectively.

\section{D.7 Uncertainty in $q_{\text {net }}$}

The least square uncertainty in $q_{\text {net }}$ is,

$$
U_{q_{\text {net }}}^{2}=U_{q}^{2}+U_{q_{\text {side }}}^{2}+U_{q_{\mathrm{lb}}}^{2}
$$

First, the uncertainty in the main heater power needs to be calculated. Recalling the definition of the heater power, 


$$
q=\frac{V^{2}}{R_{\mathrm{eff}}}
$$

the uncertainty in the main heater power can be written,

$$
\begin{aligned}
U_{q}^{2} & =\left(\frac{\partial q}{\partial V} U_{V}\right)^{2}+\left(\frac{\partial R_{\mathrm{eff}}}{\partial R_{\mathrm{eff}}} U_{R_{\mathrm{eff}}}\right)^{2} \\
& =\left(\frac{2 q}{V} U_{V}\right)^{2}+\left(\frac{q}{R_{\mathrm{eff}}} U_{R_{\mathrm{eff}}}\right)^{2}
\end{aligned}
$$

Voltage measurement in the present study are made with the Agilent 3907A digital multiplexer which according to its manual has an uncertainty of $\pm 0.6 \%$ for AC voltage measurements. This is the assumed value of relative uncertainty in voltage measurements. The relative uncertainty in the effective heater resistance is,

$$
\begin{aligned}
\left(\frac{U_{R_{\mathrm{eff}}}}{R_{\mathrm{eff}}}\right)^{2} & =\left(\frac{\partial R_{\mathrm{eff}}}{\partial R_{1}} U_{R_{1}}\right)^{2}+\left(\frac{\partial R_{\mathrm{eff}}}{\partial R_{2}} U_{R_{2}}\right)^{2} \\
& =\left(\frac{R_{\mathrm{eff}}}{R_{1}} \frac{U_{R_{1}}}{R_{1}}\right)^{2}+\left(\frac{R_{\mathrm{eff}}}{R_{2}} \frac{U_{R_{2}}}{R_{2}}\right)^{2} .
\end{aligned}
$$

The main heater resistances are measured with Radioshack 46-Range Digital Multimeter. According to its owner's manual, the multimeter reads resistances with an accuracy of $\pm 0.4 \%$. This is the assumed value of the relative uncertainty of resistance measurements.

To calculate the uncertainties in the side wall and base heat losses, the definitions of $q_{\text {side }}$ and $q_{\mathrm{lb}}$ given in equations (D.7) and (D.8) are used. Based on these definitions,

$$
\begin{gathered}
U_{q_{\text {side }}}^{2}=8 \times\left(\frac{U_{T}}{R_{\text {side }}}\right)^{2}, \\
U_{q_{\mathrm{lb}}}^{2}=\left(\frac{A_{\text {tot }} k_{\text {base }}}{w_{\text {base }}}\right)^{2}\left(U_{T_{H, \text { avg }}}^{2}+U_{T_{G, \text { avg }}}^{2}\right),
\end{gathered}
$$

where $U_{T_{G \text {,avg }}}$ is the uncertainty in average guard heater temperature. 